\title{
Treating phantom limb pain following amputation
}

Citation for published version (APA):

Rothgangel, A. S. (2019). Treating phantom limb pain following amputation: the potential role of a traditional and teletreatment approach to mirror therapy. [Doctoral Thesis, Maastricht University]. Gildeprint Drukkerijen. https://doi.org/10.26481/dis.20190625ar

Document status and date:

Published: 01/01/2019

DOI:

10.26481/dis.20190625ar

Document Version:

Publisher's PDF, also known as Version of record

\section{Please check the document version of this publication:}

- A submitted manuscript is the version of the article upon submission and before peer-review. There can be important differences between the submitted version and the official published version of record.

People interested in the research are advised to contact the author for the final version of the publication, or visit the DOI to the publisher's website.

- The final author version and the galley proof are versions of the publication after peer review.

- The final published version features the final layout of the paper including the volume, issue and page numbers.

Link to publication

\footnotetext{
General rights rights.

- You may freely distribute the URL identifying the publication in the public portal. please follow below link for the End User Agreement:

www.umlib.nl/taverne-license

Take down policy

If you believe that this document breaches copyright please contact us at:

repository@maastrichtuniversity.nl

providing details and we will investigate your claim.
}

Copyright and moral rights for the publications made accessible in the public portal are retained by the authors and/or other copyright owners and it is a condition of accessing publications that users recognise and abide by the legal requirements associated with these

- Users may download and print one copy of any publication from the public portal for the purpose of private study or research.

- You may not further distribute the material or use it for any profit-making activity or commercial gain

If the publication is distributed under the terms of Article $25 \mathrm{fa}$ of the Dutch Copyright Act, indicated by the "Taverne" license above, 


\section{TREATING PHANTOM LIMB PAIN FOLLOWING AMPUTATION}

The potential role of a traditional and teletreatment approach to mirror therapy

ANDREAS ROTHGANGEL 2019 
TREATING PHANTOM LIMB PAIN FOLLOWING AMPUTATION: The potential role of a traditional and teletreatment approach to mirror therapy 
The research presented in this thesis was conducted at: The School for Public Health and Primary Care (CAPHRI), Department of Rehabilitation Medicine, Maastricht University.

CAPHRI participates in the Netherlands School of Primary Care Research (CaRe), acknowledged by the Royal Dutch Academy of Science

(KNAW) CAPHRI was classified as excellent by the external evaluation committee of leading international experts that reviewed CAPHRI

December 2010

TREATING PHANTOM LIMB PAIN FOLLOWING AMPUTATION:

and

The Research Centres "Autonomy and Participation of Persons with a Chronic Illness" and "Nutrition, Lifestyle and Exercise", Faculty of Health, Zuyd University of Applied Sciences, Heerlen, the Netherlands.

The research presented in this thesis was funded by by the State of North Rhine-Westphalia (NRW, Germany) and the European Union through the NRW Ziel2 Programme as a part of the European Regional Development Fund (grant no. 005-GW02-035) and by Zuyd University of Applied Sciences

The printing of this thesis was financially supported by the Scientific College Physical Therapy (WCF) of the Royal Dutch Society for Physica Therapy (KNGF)

(๑) Andreas Rothgangel, Maastricht 2019

All rights reserved. No parts of this thesis may be reproduced or transmitted in any form or by any means, without prior permission in writing by the author, or when appropriate, by the publishers of the publications.

Art work: Matthias Reinhold (mail@matthiasreinhold.de)

Lay-out: Carolin Trzaskowski

Printed by: Gildeprint, Enschede

ISBN: 9789463236973

PROEFSCHRIFT

ter verkrijging van de graad van doctor

op gezag van de Rector Magnificus, Prof. dr. Rianne M. Letschert volgens het besluit van het College van Decanen,

in het openbaar te verdedigen op

Andreas Rothgange 
Promotores

Prof. dr. R.J.E.M. Smeets

Prof. dr. A.J.H.M. Beurskens

Co-promotor

Dr. S.M. Braun

Assessment committee

Prof. dr. G.M. Rommers (chair) (Maastricht University)

Prof. dr. J.W.S. Vlaeyen (Maastricht University, University of Leuven)

Prof. dr. K. Horstmann (Maastricht University)

Prof. dr. JowC van Gemert-Pinen (University of Twente, Enschede)

Prof. d. J.EWC. van Gemert-Pinen (Universiy of Twente, Enschede)

Dr. U. Polak (German Social Accident Insurance (DGUV) Berlin, Germany) 



\section{CONTENTS}

CHAPTER 1 General Introduction

CHAPTER 2 The clinical aspects of mirror therapy in rehabilitation: a systematic review of the literature International Journal of Rehabilitation Research, 2011;34(1):1-13.

CHAPTER 3 Development of a clinical framework for mirror therapy in patients with phantom limb pain: (2) Pain Practice, 2016;16(4): 222-34.

CHAPTER 4 Design and development of a telerehabilitation platform for patients with phantom limb pain: user-centered approach

Journal of Medical lnternet Research Rehabilitation and Assistive Technologies, 2017.4(1).e2.

CHAPTER 5 The PACT trial: PAtient Centered Telerehabilitation: Effectiveness of software-supported and aditional mirror therapy in patients with phantom limb pain following lower limb amputation:

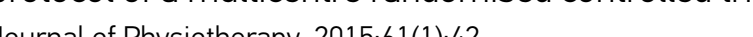

CHAPTER 6 Traditional and augmented reality mirror therapy for patients with chronic phantom limb pain (PACT study): results of a three-group, multicentre single-blind randomized controlled trial Clinical Rehabilitation, 2018:32(12):1591-1608

CHAPTER 7 Feasibility of a traditional and teletreatment approach to mirror therapy in patients with phantom alion. a process evaluation performed alongside a randomized controlled trial Clinical Rehabilitation, 2019; May

CHAPTER 8 General Discussion

Summary

Samenvatting

Zusammenfassung

Valorisation

Acknowledgements / Dankwoord / Danksagung

About the author

List of publications
185

223

293

301

309

327

341 



\section{GENERAL INTRODUCTION}

Chronic pain affects one out of five adults in Europe' and its prevalence increases with age. ${ }^{2}$ Chronic pain severely impairs patients' in their daily lives. It is responsible for considerable limitations in work and quality of life and leads to a significant increase in healthcare costs. ${ }^{3.4}$ Chronic pain accompanies many different chronic conditions such as musculoskeletal disorders, which are amongst the 10 highest-ranking conditions worldwide regarding the amount of years lived with disability. ${ }^{.}$. Furthermore, $7 \%$ of adults in the general population suffer from severe chronic pain and anoher 7-10\% have neuropathic pain caused by damage or disease affecting the somatosensory nervous system. ${ }^{6}$

\section{Phantom limb pain}

Whin the group of patients with chronic neuropathic pain, phantom limb pain following amputation is frequent and affects up to $80 \%$ of amputees. ${ }^{\prime 8}$ This pain is perceived in the entire or parts of the missing limb and varies in character from sharp, shooting pain to sensations similar to an electric shock or dull, squeezing or cramping forms as shown in figure

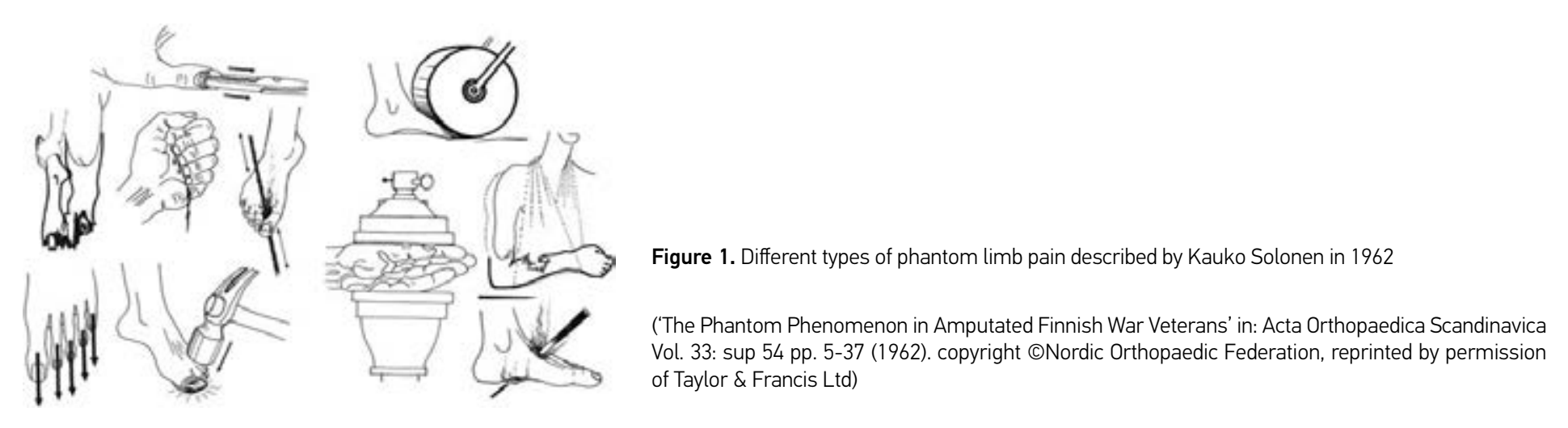

One study $y^{7}$ reported that a large proportion (38.9\%) of amputees experiences severe phantom limb pain defined as scoring a 7 or higher on the 11-point Numeric Rating Scale. The occurrence of phantom limb pain seems not to depend on individual patient characteristics such as age, gender or level and side of amputalon

In the majority (75\%) of patients, phantom limb pain occurs within the first days after amputation. ${ }^{1{ }^{1-13}}$ However, single cases are described in which the pain first appeared several years after the amputation.14 Regarding the duration of phantom limb pain one study suggested that phantom limb pain is decreasing over time, ${ }^{15}$ whereas another study showed no decrease or even an increase in phantom limb pain ${ }^{13}$ Several prospective studies showed that the majority of amputees suffers from phantom limb pain several years after the amputation 10 A large survey in 400 amputees 16 showed that almost half the patients (43.9\%) perceived phantom limb pain more than 5 hours dily and 27 7\% reported constant pain Patients report that the pain apea watching TV or sleeping:

I am suffering from phantom limb pain since my accident 30 years ago. My phantom leg feels like a big often wake up at night because this clump starts cramping and stabbing. Then I am walking around and trying to relieve the pain by rubbing the stump or by increasing med
dose. However, with this amount of medication, I better stay in bed the next day.

- Thomas, 52 years, patient representative PACT project

Sensations in the phantom limb following amputation have first been medically described in the mid-1 6th century by French military surgeon Ambroise Paré. 17 who observed that patients complained of severe pain in the missing limb.

The American Neurologist Silas Weir Mitchell was the first to use the term 'phantom limb' and to describe different phantom limb sensations in more detail in the chapter "Neural Maladies of Stumps" of his famous textbook "Injuries of nerves and their consequences." ${ }^{.8}$ 
Interestingly, some of Mitchell's observations described in 1872, e.g. the prevalence rate of phantom limb sensations such as telescoping are still consistent with current scientific data. ${ }^{19}$ At the time, Mitchell published his observations on hundreds of amputees, phantom limb pain and other sensations were regarded as mental hallucinations. However, over 100 years later, the view on phantom limb pain has not much changed. A study ${ }^{20}$ from 1983 reported that only a small proportion of patients (17\%) who discussed the phantom limb pain with their doctor were offered treatment, and a large proportion were told that they were mentally disturbed. Similar results were reported in a study from 1997 by Wartan et al..21 in which one third of patients reporting phantom pain to their doctor were told that their pain was imaginary and either would go away without further treatment or never. Despite the fact that phantom limb pain has already been known for hundreds of years and has a major impact on patients' life, treatments achieving sustainable effects are still lacking.

\section{Neurophysiological mechanisms of phantom limb pain}

One potential explanation for the fact that effective treatments against phantom limb pain are lacking might be that these treatments do not effectively target its underlying cause Besides changes in the peripheral nervous system such as ectopic discharge from a stump neuroma ${ }^{22}$ central mechanisms on the spinal and supraspinal level have been proposed to be associated with the occurrence of phantom limb pin $^{23}$ In 1991, a study in adult macaques by Pons et al.2.4 found that after long-term deafferentation of a limb, the cortical area of the deafferentated limb became responsive to stimuli applied to the neighbouring cortical area of the face region. These findings were confirmed one year later

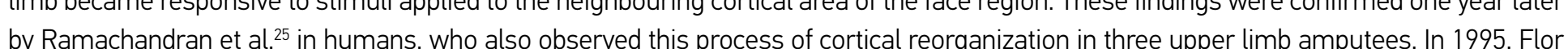
and colleagues published the fis (a) the cortical representation of the amputated limb contributes to the occurrence and maintenance of phantom limb pain.272-29

\section{Treatment of phantom limb pain}

A publication from $1980^{30}$ already identified 43 different treatment modalities that were used to treat phantom limb pain, and in the A following years many other interventions such as pharmacologic or comples comprises different types of pain medication ranging from more generic drugs such as Paracetamol to stronger painkillers such as opioids However, patients frequently complain about negative side effects, and for opioids a significant addiction potential has been proven $32.33 \mathrm{In}$. addition, opioids are only recommended as a third line treatment, ${ }^{33}$ since the quality of evidence regarding its long-term efficacy is only low to moderate $e^{33,34}$ whereas costs are high. In this context, alternative, nonpharmacological interventions have gained increasing attention in the treatment of phantom limb pain during the past years. These strategies range from hypnosis, sensory discrimination training on the stump to residual limb liners made from electromagnetic shielding fabric containing fine steal fibres. ${ }^{35,36} \mathrm{~A}$ study by Lotze et al..$^{28}$ suggested that frequent use of a myoelectric arm prosthesis also has beneficial effects on phantom limb pain. This suggestion is clinically confirmed by physical and occupational therapists treating amputees during rehabilitation, who anecdotally reported hat gait training using the prosthesis has positive effects on phantom limb pain.

Nonetheless, therapeutic interventions that effectively target phantom limb pain are limited In light of the central malplasticity described above, movement representation techniques such as mental pracice or mirror therapy that target these central mechanisms offer promising new possibilities for therapists to treat phantom limb pain. ${ }^{37,38}$

\section{Mirror therapy in rehabilitation}

The principle of mirror therapy was first described in 1995 by Ramachandran and colleagues ${ }^{39}$ and aimed to facilitate motor control of the phantom limb and to relieve phantom pain in nine upper limb amputees in seven out of nine patients, observing movements of the intact hand in the mirror resulted in increased feeling of movements of the phantom hand In addition. five patients experienced painful cramps in the phantom hand, which could be relieved by watching the mirror reflection of both hands opening simultaneously. Interestingly, the increased feeling of movement and the relief of spasms in the phantom hand was paralled by a reduction in phantom limb pain. During mirror therapy, the patient sits in front of a mirror that is oriented parallel to the patients' mid limb By looking into the mirror, the visual illusion of two intact limbs is created (Figure 2). which can be used to reduce pain or to facilitate motor function of the affected limb.

Soon after the first reports in patients with phantom limb pain, mirror therapy was also applied to stroke patients to enhance motor function So the paralyzed limb 41 Since then, most of the research on miror the the fact that promising results were also found in patients with complex regional pain syndrome ${ }^{42}$ and phantom limb pain. ${ }^{43}$ 


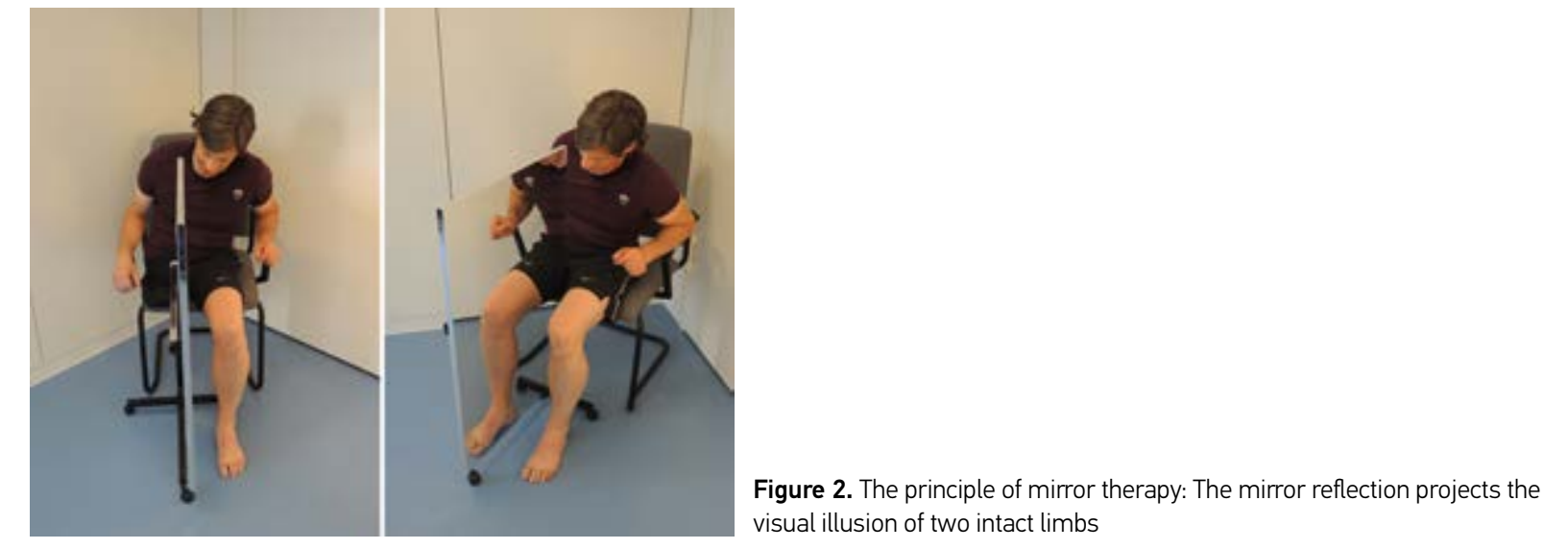

Ten years after the first study by Ramachandran et al.,.39 who used the mirror box in upper limb amputees, another study also applied the mirror box to 21 lower limb amputees. This study confirmed that mirror therapy enhanced motor control over the phantom leg, as had already been suggested by Ramachandran for the upper extremity. The first randomized controlled trial including a mixed sample of patients with complex regional pain syndrome, brachial plexus avulsion and amputation that suggested positive effects of mirror therapy on phantom limb pain was published by Moseley in 2006.45 Neurophysiological studies suggested that these positive effects of mirror therapy on phantom limb pain might be explained by normalization of central malplasticity.66,47

\section{Inconsistency how to perform mirror therapy and limited evidence}

Since the first publication on mirror therapy in amputees, ${ }^{39}$ different methods of how to perform mirror therapy in patients with phantom limb pain have been described, ranging from a combination of limb laterality recognition training, mental practice and mirror therapy, 45 to solely using mirror therapy.43 Despite the potential merits of mirror therapy, almost 20 years after the first publication on mirror therapy in patients with phantom limb pain, evidence for its effectiveness is still low. Only two controlled studies including a total of 27 amputees s.3. $^{2}$ 45 are published that reported positive effects on phantom limb pain. Furthermore, little is known about important patient and intervention characteristics, and a clear description of how to successfully implement mirror therapy in daly care is missing. Thus, existing interventions with mirror therapy seem not to be comparable, since many variations in mirror therapy exist and little is known about important clinical aspects of the intervention In addition, occupational and physical therapists treating patients with phantom limb pain need tools to support standardized implementation of mirror therapy in clinical practice.

\section{The need to develop and evaluate a user-centred telerehabilitation}

Given the chronic nature of phantom limb pain and suggested central malplasticity, it was proposed that patients should self-deliver mirror therapy long-term to achieve sustainable effects. Besides this aspect, the growing financial pressures on the health care system due to an ageing society shifted the focus in the last years more and more towards self-monitoring and self-management of patients. However research pointed out that adherence to unsupervised exercises is generally poor and additional tools and strategies are necessary to support long-term self-management of patients. ${ }^{48}$

In 1998, the first article on the use of telerehabilitation was published and followed by many other studies in the field, which suggest teletreatments as a promising tool to support patients' self-management and self-efficacy.99 Studies showed that teletreatments are able to increase exercise acherence ${ }^{50}$ and that patients took greater responsibility for their own health when they were able to see their own health data. ${ }^{51}$ In addition, given the technological advancements in the recent years, novel technology-driven interventions such as augmented or virtual reality were developed and applied in patients with phantom limb pain. ${ }^{52}$ However, despite the fact that these novel interventions offer promising new possibilities to treat patients with phantom limb pain no controlled studies investigating effects have been published so far Furthermore many novel teletreatments are not accepted by their users because the technologies are often not developed with sufficient (end-) user engagement ${ }^{53}$ Such technologies have to match with people's dily lives, habits or routines, if they want to create sufficient ence participary dus

(a) ( (1) different aspects besides the delive process evaluation to gain more in professionals At this point our research project satednine years ago, in 2010 . 


\section{Aim of the thesis}

The main aim of his project was to develop a clinical framework for mirror therapy as well as a user-centered teletreatment using augmented reality mirror therapy and to evaluate their feasibility and effects in patients with phantom limb pain following lower limb amputation.

Within this project, three phases can be distinguished to reach the central aim of the project: First, a theoretical foundation was developed to deliver mirror therapy in clinical practice.

The objective of the first phase was to conduct a systematic review of the literature regarding important clinical aspects and the quality of evidence of applying mirror therapy in patients with stroke, complex regional pain syndrome and phantom limb pain.

This theoretical foundation then served as a starting point in phase two of the project to model a clinical framework for mirror therapy and a novel telerehabilitation platform.

The aim of the second phase was to design and develop a clinical framework and a user-centered telerehabilitation platform for mirror therapy in patients with phantom limb pain following lower limb amputation.

The feasibility and effects of the clinical framework and the novel teletreatment were then evaluated in phase three of the project.

The aim of the third phase was to evaluate the effects of the clinical framework for mirror therapy and the additional effects of a teletreatment using augmented reality mirror therapy in patients with phantom limb pain. It was to also investigate whether the interventions were delivered by patients and therapists as expected.

\section{Outline of the thesis}

Figure 3 provides an overview of the various chapters and gives an outline of the thesis.

Chapter 2 describes the theoretical foundation and how important clinical aspects and the evidence base of mirror therapy were identified.

Chapter 3 presents the development and content of a clinical framework for mirror therapy in patients with phantom limb pain based on the best available evidence, patient preferences and clinical expertise of physical and occupational therapists. The framework illustrates important patient and intervention characteristics and can be used to personalize mirror therapy in daily care.
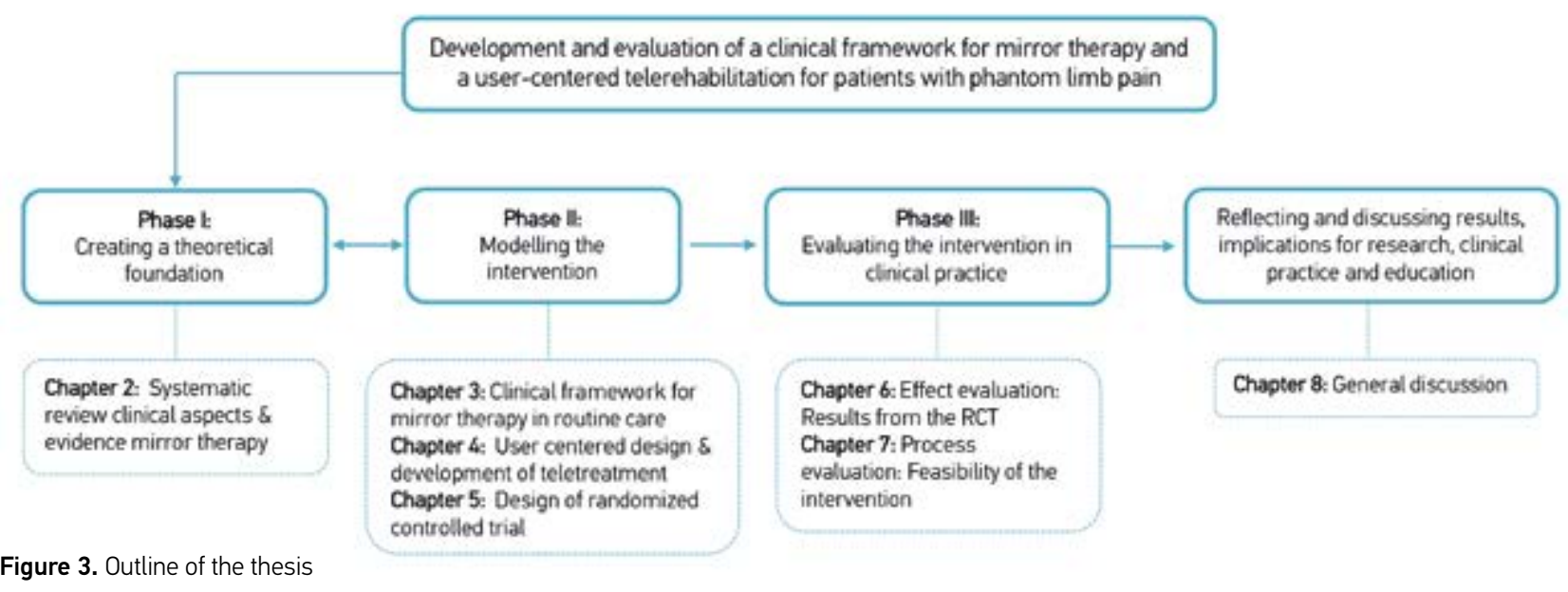

Chapter 4 illustrates the user-centered approach that guided the design and development of the telerehabilitation platform for patients

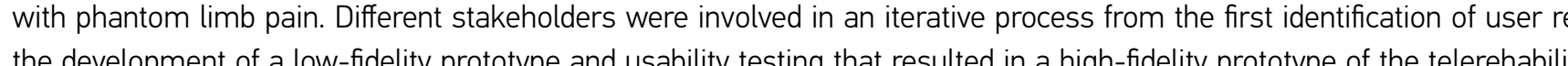
After the interventions had been modelled, a three-group multicentre randed in a high-fidelity prototype of the telerehabilitation plattorm. the clinical framework for mirror therapy and the additional value of the teletreatment. During the preparation of the trial several questions concerning the study design emerged. Chapter 5 explains the trial design. shows how these questions were addressed and evaluates the arcuments for the choices made. The results from this trial regarding the effects of the interventions are reported in $\mathrm{Chapter} 6$. We decided a priori to also perform a detaled process evaluation of the trial as shom in Che 7 . In particular in multicentre trials investigating (nials investigating are discussed and implications for research, clinical practice and education of future health care professionals are explored. 


\section{REFERENCES}

Breivik H, Collett B, Ventafridda V, Cohen R and Gallacher D. Survey of chronic pain in Europe: prevalence. impact on daily life, and treatment. Eur J Pain. 2006; 10: 287-333.

2. Elliott AM, Smith BH, Penny KI, Smith WC and Chambers WA. The epidemiology of chronic pain in the community. Lancet. 1999; 354: 1248-52.

3. Andrew R, Derry S, Taylor RS, Straube $S$ and Phillips $C J$. The costs and consequences of adequately managed chronic non-cancer pain and chronic neuropathic pain. Pain Pract. 2014; 14: 79-94.

Patel AS, Farquharson R, Carroll D, et al. The impact and burden of chronic pain in the workplace: a qualitative systematic review. Pain Pract. 2012; 12: 578-89.

5. Global Burden of Disease Study 2013 Collaborators. Global, regional, and Global, regional, and national incidence, prevalence, and years lived with disability for 301 acute and chronic diseases and injuries in 188 countries, 1990-2013: a systematic analysis for the Global Burden of Disease Study 2013. Lancet. 2015; 386: 743-800

6. van Hecke O, Austin SK, Khan RA, Smith BH and Torrance N. Neuropathic pain in the general population: a systematic review of epidemiological studies. Pain. 2014; 155: 654-62.

7. Ephraim PL, Wegener ST, Mackenzie EJ, Dillingham TR and Pezzin LE. Phantom pain, residual limb pain, and back pain in amputees: results of a national survey. ArchPhys Med Rehabil. 2005; 86: 1910-9.

8. Richardson C, Glenn S, Nurmikko Tand Horgan M. Incidence of phantom phenomena including phantom limb pain 6 months after major lower limb amputation in patients with peripheral vascular disease. Clin J Pain. 2006; 22: 353-8.

9. Hsu E and Cohen SP. Postamputation pain: epidemiology, mechanisms, and treatment. J Pain Res. 2013; 6:121-36.
10. Nikolajsen L and Jensen TS. Phantom limb pain. Br J Anaesth. 2001; 87: 107-16.

11. Carlen PL, Wall PD, Nadvorna H and Steinbach T. Phantom limbs and related phenomena in recent traumatic amputations. Neurology. 1978; 28: 211-7.

12. Jensen TS, Krebs B, Nielsen J and Rasmussen P. Phantom limb, phantom pain and stump pain in amputees during the first 6 months following limb amputation. Pain. 1983; 17: 243-56.

13. Nikolajsen L, Ilkjaer S, Kroner K, Christensen JH and Jensen TS. The influence of preamputation pain on postamputation stump and phantom pain. Pain. 1997; 72: 393-405

14. Raibhandari SM, Jarratt JA, Griffiths PD and Ward JD. Diabetic neuropathic pain in a leg amputated 44 years previously. Pain. 1999; 83: 627-9.

15. Houghton AD, Nicholls G, Houghton AL, Saadah E and MCColl L. Phantom pain: natural history and association with rehabilitation. Ann R Coll Surg Engl. 1994; 76: 22-5.

16. Kern U, Busch V, Rockland M, Kohl M and Birklein F. Prevalence and risk factors of phantom limb pain and phantom limb sensations in Germany. A nationwide field surveyl. Schmerz. 2009; 23: 479-88.

17. Keil G. Sogenannte Erstbeschreibung des Phantomschmerzes von Ambroise Paré. Fortsch Med. 1990; 108: 62-6.

18. Mitchell SW. Injuries of nerves and their consequences. Philadelphia: J.B. Lippincott, 1872

19. Montoya P, Larbig W, Grulke N, Flor H. Taub E and Birbaumer N. The relationship of phantom limb pain to other pha limb he

20. Sherman RA and Sherman CJ. Prevalence and characteristics of chronic phantom limb pain among American veterans. Results of a trial survey. Am J Phys Med. 1983; 62: 227-38.

21. Wartan SW, Hamann W, Wedley JR and McColl I . Phantom pain and sensation among British veteran amputees. Br J Anaesth. 1997; 78: 652-9.

22. Wall PD and Gutnick M. Ongoing activity in peripheral nerves: the physiology and pharmacology of impulses originating from a neuroma. Exp Neurol. 1974: 43: 580-93. 
23. Flor H. Phantom-limb pain: characteristics, causes, and treatment. Lancet Neurol. 2002; 1: 182-9.

24. Pons TP, Garraghty PE, Ommaya AK, Kaas JH, Taub E and Mishkin M. Massive cortical reorganization after sensory deafferentation in adult macaques. Science. 1991; 252: 1857-60.

25. Ramachandran VS Rogers-Ramachandran D and Stewart M. Perceptual correlates of massive cortical reorganization. Science. 1992; 258: 1159-60.

Flor $\mathrm{H}$, Elbert T, Knecht S, et al. Phantom-limb pain as a perceptual correlate of cortical reorganization following arm amputation. Nature. 1995; 375: 482-4

27. Knecht S, Henningsen $\mathrm{H}$, Hohling $\mathrm{C}$, et al. Plasticity of plasticity? Changes in the pattern of perceptual correlates of reorganization after amputation. Brain. 1998; 121(Pt 4): 717-24

28. Lotze M, Grodd W, Birbaumer N, Erb M, Huse E and Flor H. Does use of a myoelectric prosthesis prevent cortical reorganization and phantom limb pain? Nat Neurosci. 1999; 2: 501-2.

29. Lotze M, Flor $\mathrm{H}$, Grodd W, Larbig W and Birbaumer N. Phantom movements and pain. An fMRI study in upper limb amputees. Brain. 2001; 124: 2268-77.

30. Sherman RA. Published treatments of phantom limb pain. Am J Phys Med. 1980; 59: 232-44.

31. Halbert J, Crotty M and Cameron ID. Evidence for the optimal management of acute and chronic phantom pain: a systematic review. Clin J Pain. 2002; 18: 84-92.

McNicol ED, Midbari A and Eisenberg E. Opioids for neuropathic pain. Cochrane Database Syst Rev. 2013: CD006146.

33. Finnerup NB, Attal N, Haroutounian S, et al. Pharmacotherapy for neuropathic pain in adults: a systematic review and meta-analysis. Lancet Neurol. 2015; 14: 162-73.

Alviar MJ, Hale T and Dungca M. Pharmacologic interventions for treating phantom limb pain. The Cochrane database of systematic reviews. 2016; 10: CD006380.

35. Batsford S, Ryan CG and Martin DJ. Non-pharmacological conservative therapy for phantom limb pain: A systematic review of randomized controlled trials. Physiother Theory Pract. 2017: 33: 173-83.
36. Flor H, Denke C, Schaefer M and Grusser S. Effect of sensory discrimination training on cortical reorganisation and phantom limb pain. Lancet. 2001; 357: 1763-4.

37. Maclver K, Lloyd DM, Kelly S, Roberts $\mathrm{N}$ and Nurmikko T. Phantom limb pain, cortical reorganization and the therapeutic effect of mental imagery. Brain. 2008; 131: 2181-91

38. Giraux P and Sirigu A. Illusory movements of the paralyzed limb restore motor cortex activity Neuroimage. 2003; 20 Suppl 1: S107-S11.

39. Ramachandran VS, Rogers-Ramachandran D and Cobb S. Touching the phantom limb. Nature. 1995; 377: 489-90

40. Thieme H. Mehrholz J. Pohl M. Behrens J and Dohle C. Mirror therapy for improving motor function after stroke. Cochrane Database Syst Rev. 2012: CD008449.

1. Altschuler EL, Wisdom SB, Stone L, et al. Rehabilitation of hemiparesis after stroke with a mirror. Lancet 1999; 353: 2035-6.

42. Moseley GL. Graded motor imagery is effective for long-standing complex regional pain syndrome: a randomised controlled trial. Pain. 2004; 108: 192-8.

3. Chan BL, Witt R, Charrow AP, et al. Mirror therapy for phantom limb pain. N Engl J Med. 2007; 357: 2206-7,

4. Brodie EE. Whyte $A$ and Waller $B$. Increased motor control of a phantom leg in humans results from the visual feedback of a virtual leg. Neurosci Lett. 2003; 341: 167-9

Moseley GL. Graded motor imagery for pathologic pain: a randomized controlled trial. Neurology. 2006; 67: 2129-34.

46. Giraux P and Sirigu A. Illusory movements of the paralyzed limb restore motor cortex activity. Neuroimage. 2003; 20 Suppl 1: S107-11.

4. Foell J, Bekrater-Bodmann R, Diers M and Flor H. Mirror therapy for phantom limb pain: brain changes and the role of body representation. Eur J Pain. 2014; 18: 729-39. 
48. Darnall BD and Li H. Home-based self-delivered mirror therapy for phantom pain: a pilot study. J Rehabil Med. 2012; 44: 254-60.

49. Rogante M, Grigioni M, Cordella D and Giacomozzi C. Ten years of telerehabilitation: A literature overview of technologies and clinical applications. NeuroRehabilitation. 2010; 27: 287-304.

50. Huis in 't Veld RM, Kosterink SM, Barbe T, Lindegard A, Marecek T and Vollenbroek-Hutten MM. Relation between patient satisfaction, compliance and the clinical benefit of a teletreatment application for chronic pain. J Telemed Telecare. 2010; 16: 322-8.

51. Kidholm K, Stafylas P, Kotzeva A, et al. Renewing Health: REgioNs of Europe WorklNg toGether for HEALTH. 2014.

52. Dunn J, Yeo E, Moghaddampour P, Chau B and Humbert S. Virtual and augmented reality in the treatment of phantom limb pain: A literature review. NeuroRehabilitation. 2017; 40: 595-601.

53. Broens TH, Huis in't Veld RM, Vollenbroek-Hutten MM, Hermens HJ, van Halteren AT and Nieuwenhuis LJ. Determinants of successful telemedicine implementations: a literature study. J Telemed Telecare. 2007; 13: 303-9.

54. Eysenbach G. Medicine 2.0: social networking, collaboration, participation, apomediation, and openness. J Med Internet Res. 2008; 10: e22.

55. Huis in 't Veld RM, Widya IA, Bults RG, Sandsjo L, Hermens HJ and Vollenbroek-Hutten MM. A scenario guideline for designing new teletreatments: a multidisciplinary approach. J Telemed Telecare. 2010; 16: 302-7.

56. van Gemert-Pijnen JE, Nijland N, van Limburg M, et al. A holistic framework to improve the uptake and impact of eHealth technologies. J Med Internet Res. 2011; 13: e111.

57. Saunders RP, Evans MH and Joshi P. Developing a process-evaluation plan for assessing health promotion program implementation: a how-to guide. Health Promot Pract. 2005; 6: 134-47.
58. Oakley A, Strange V, Bonell C, Allen E, Stephenson J and Team RS. Process evaluation in randomised controlled trials of complex interventions. BMJ. 2006; 332: 413-6. 


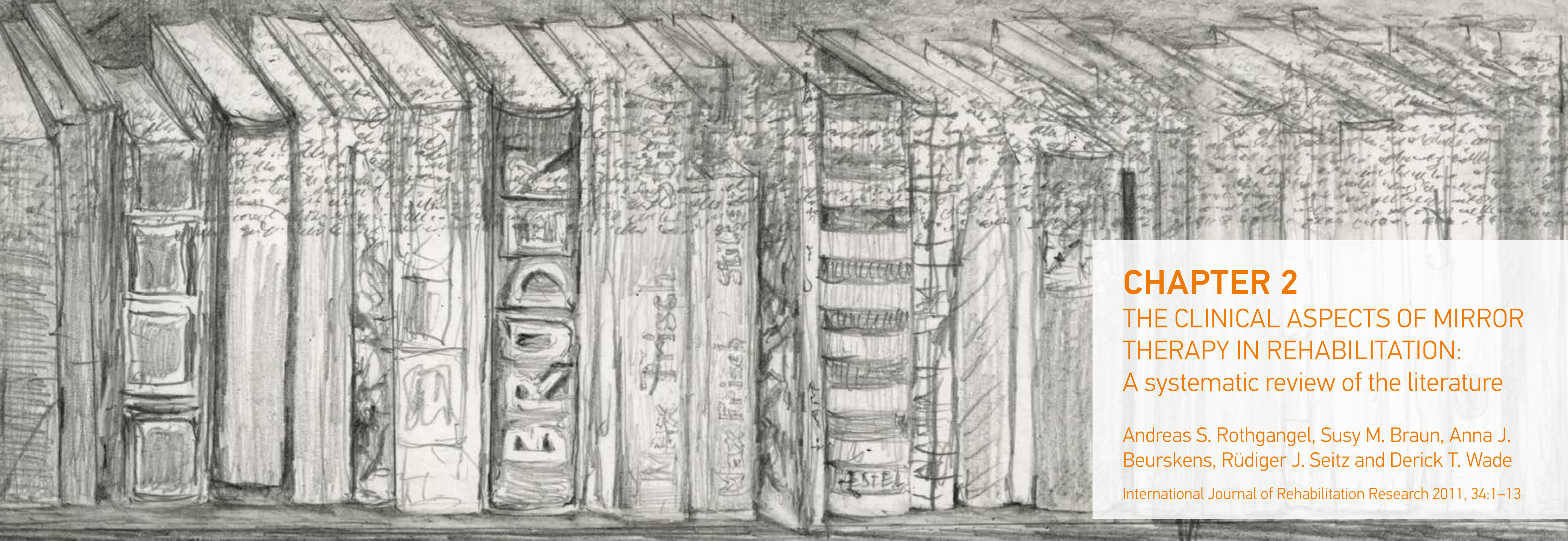




\section{ABSTRACT}

The objective of this study was to evaluate the clinical aspects of mirror therapy (MT) interventions after stroke, phantom limb pain and complex regional pain syndrome. A systematic literature search of the Cochrane Database of controlled trials, PubMed/MEDLINE, CINAHL, EMBASE, PsycINFO, PEDro, RehabTrials and Rehadat, was made by two investigators independently (A.S.R. and M.J.). No restrictions were made regarding study design and type or localization of stroke, complex regional pain syndrome and amputation. Only studies that had MT given as a long-term treatment were included. Two authors (A.S.R. and S.M.B.) independently assessed studies for eligibility and risk of bias by using the Amsterdam-Maastricht Consensus List. Ten randomized trials, seven patient series and four single-case studies were included. The studies were heterogeneous regarding design, size, conditions studied and outcome measures. Methodological quality varied; only a few studies were of high quality. Important clinical aspects, such as assessment of possible side effects, were only insufficiently addressed. For stroke there is a moderate quality of evidence that $\mathrm{MT}$ as an additional intervention improves recovery of arm function, and a low quality of evidence regarding lower limb function and pain after stroke. The quality of evidence in patients with complex regional pain syndrome and phantom limb pain is also low. Firm conclusions could not be drawn. Little is known about which patients are likely to beneft most from Outcome measures and systematically register adverse effects.

\section{INTRODUCTION}

In mirror thrapy (MT), the patient sits in front of a mirror that is oriented parallel to his midline blocking the view of the (affected) limb positioned behind the mirror. When looking into the mirror, the patient sees the reflection of the unaffected limb positioned as the affected

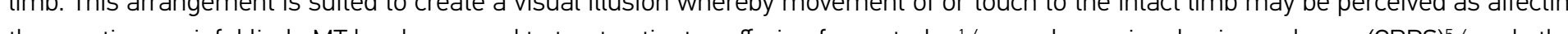

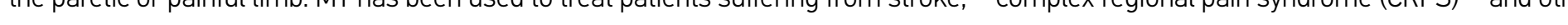
pain syndromes such as peripheral nerve injury and follow most are stroke, CRPS and phantom limb pain (PLP)?
The underlying mechanisms of the effects in these three patient groups have mainly been related to the activation of 'mirror neurones', which may also be activated when observing others perform movements and also during mental practice of motor tasks. 10.11 Mirror neurons were found in areas of the ventral and inferior premotor cortex associated with observation and imitation of movements and in somatosensory cortices associated with observation of touch. ${ }^{12-14}$ These cortical areas are supposed to be activated by MT.15,16 Until now, direct evidence for the mirror-related recruitment of mirror neurons is lacking ${ }^{16-18}$ Other potential mechanisms such as enhanced self-awareness and spatial attention by activation of the superior temporal gyrus, precuneus and the posterior cingulate cortex have been proposed 16, 18, 19 The superior temporal gyrus is also thought to play an important role in recovery from neglect 20.21 and is activated by observation of biological motion .22

Recently three reviews on the topic of $\mathrm{MT}$ have been published, ${ }^{923.24}$ concentrating on the effectiveness of $\mathrm{MT}$ in different diseases. In contrast to these studies, our study focuses on the clinical aspects of $\mathrm{MT}$ interventions, which have not yet explicitly been addressed and in addition includes recently published papers. In addition, our study includes only those studies that had MT given as a long-term treatment, defined as more than two interventions. We defined 'clinical aspects' of MT interventions as a compound of clinically relevant factors that allow for reproduction of the intervention in daily practice. These include detailed information on treatment and patient characteristics, use of clinically relevant outcome measures and description of possible side effects of the intervention.

Thus, the main objective of this study was to conduct a systematic review on the clinical aspects of applying MT interventions after stroke PLP and CRPS (Fig. 1) 


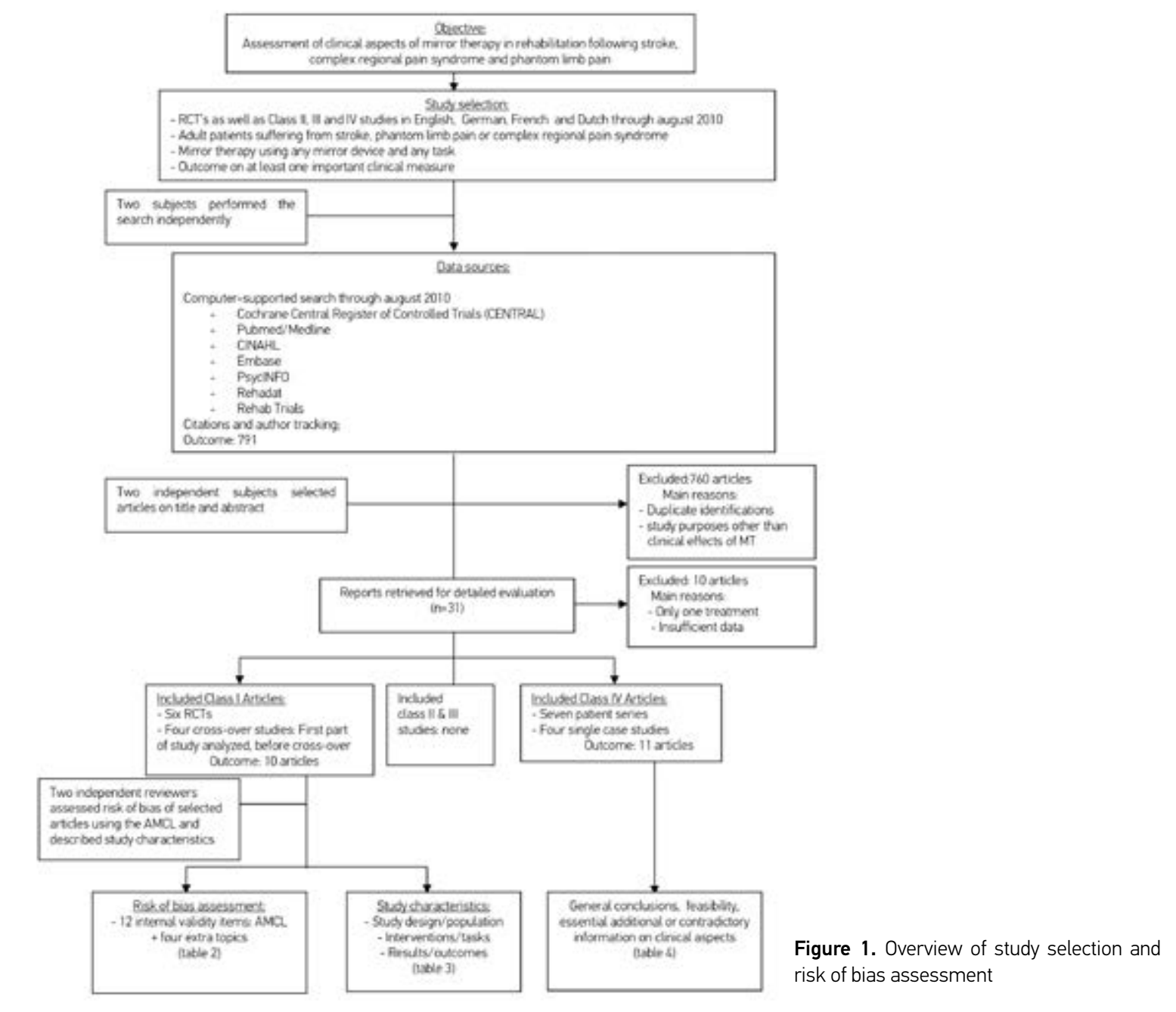

\section{S AND METHODS}

Citteria for considering studies for

Types of studies

The studies included in this review were all available articles published before August 2010 in English, German, French and Dutch. All evaluating the clinical aspects of MT were considered

The articles were categorized according to their study design.25

(1) Class I: randomized controlled studies;

Class Il: cohort studies and nonrandomized CCTs;

(3) Class III: case-control studies;

(4) Class IV: single-case studies and patient series.

\section{Types of participants}

All studies that involved adult patients (aged $>18$ years) suffering from stroke, PLP or CRPS were included. No restrictions were made with regard to the type or localization of stroke, CRPS and amputation.

Types of interventions

To be included, studies had to have MT given as a long-term treatment, defined as more than two interventions, either as the only therapy the immediate effects were excluded.

For the purpose of this study, MT was defined as the use of a mirror reflection of unaffected limb movements superimposed on the affected extremity. Therefore, studies could use a parasagittal mirror or a modified mirror device $\left(45^{\circ}\right)$ suggesting movements made by the affected limb. Other illusory mechanisms such as using immersive virtual reality were excluded. 


\section{Types of outcome measures}

According to the aim of this systematic study, trials were included only if they studied the effects of MT on at least one important clinical

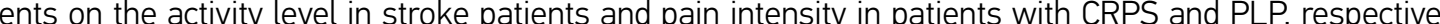
Sudies that analysed only cortical mechanisms of MT using measurements such as functional magnetic resonance imaging (FMRI) or transcranial magnetic stimulation (TMS) were excluded.

Studies were also excluded if:

(1) Only the theoretical background of MT was investigated;

Only the (conference) abstract was available.

\section{Search strategy for identification of studies}

Studies were identified by a computer-supported search through August 2010 using the following databases: Cochrane Database of controlled trials, PubMed/MEDLINE, CINAHL, EMBASE, PsycINFO, PEDro, RehabTrials and German databases such as DIMDI and Rehadat. The search strategy that was used for databases such as PubMed and Cochrane served as the main protocol and was then modified for searching other databases.

The following keywords were used: imagery, mirror, feedback/psychological, rehabilitation, therapy, stroke, amputation, phantom limb, dhe detaled search strategies are available on request from the first investigator (A.S.R.)

Additional methods used included screening of the reference lists of identified articles, search on the investigators of identified studies and personal communication with experts in the field of $M T$.
Data collection and analysis

All sources were searched independently by two investigators [A.S.R. (researcher) and Marsha Jussen (librarian)] by applying the stated selection criteria Disagreement with regard to the study selection was resolved by consensus, and in the case of persisting disagreement a third investigator (S.M.B.) was consulted

Assessment of risk of bias and clinical aspects

To assess the methodological quality of included RCTs and CCTs, we used the Amsterdam-Maastricht Consensus List (AMCL) for Quality of these clinical relevance factors is also recommended by the Cochrane Back Review Group. ${ }^{28}$ Each criterion was checked for the availability of complete information and if insufficient information was given the criterion was scored as unclear ( 20 points). If sufficient information was available the criterion was scored as either positive (+, 1 point) or negative $(-, 0$ points), leading to a maximum score of 11 points per study. We defined a study to have sufficient methodological quality if the score on the AMCL was equal to or above six points 26,29 Quality items were discussed by the two investigators ( $S$ S R SMB) beforehand, and a consensus method was used to resolve disagreements. If disagreements persisted, a third review investigator (A,B) was consulted The included studies were notblinded for investigars, insitution or journal because the investigators who assessed the risk of bias were familiar with the literature.

\section{Data extraction}

Two investigators (ASR SMB) independently extracted data on study design, population interventions and outcomes using a standardized extraction form. Disagreement between the reviewers with regard to the study characteristics was resolved before data were extracted. 
RESULTS

Table 1. Risk of bias assessment of selected randomized trials with the Amsterdam-Maastricht consensus list tor quality assessment

\begin{tabular}{|c|c|c|c|c|c|c|c|c|c|c|}
\hline \multirow[b]{2}{*}{ thems } & \multicolumn{7}{|c|}{ Stroke } & \multicolumn{3}{|c|}{ CRPS and PLP } \\
\hline & $\begin{array}{l}\text { Cacchio } \\
\text { etal." }\end{array}$ & $\begin{array}{l}\text { Cacchio } \\
\text { etalcitio }\end{array}$ & $\begin{array}{l}\text { suteygzaz } \\
\text { etate }\end{array}$ & $\begin{array}{l}\text { Yavuzer } \\
\text { etale }\end{array}$ & $\begin{array}{l}\text { Dohle } \\
\text { etalt" }\end{array}$ & $\begin{array}{l}\text { Rothagaggel } \\
\text { etal. }\end{array}$ & $\begin{array}{l}\text { Allschuler } \\
\text { etall:" }\end{array}$ & Moseleye & Moseley" & Chanetal" \\
\hline 1a Method of randomization & ; & $?$ & : & : & * & $\vdots$ & \pm & + & ; & 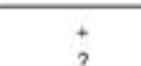 \\
\hline 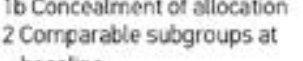 & ? & $?$ & ; & : & $\div$ & $?$ & $?$ & † & $?$ & $?$ \\
\hline 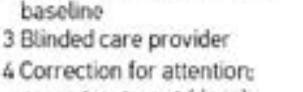 & ; & ; & ; & $;$ & $\overline{7}$ & $\mp$ & $\mp$ & $;$ & $=$ & ; \\
\hline 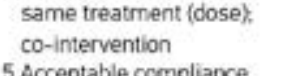 & & & & & & & & & & \\
\hline $\begin{array}{l}\text { 5Accoptatlele compliance } \\
6 \text { 6unded paternt }\end{array}$ & ? & $?$ & ¿ & $\therefore$ & $\therefore$ & ¿ & 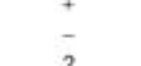 & $\div$ & $=$ & $?$ \\
\hline 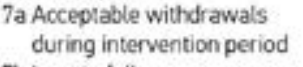 & ${ }^{*}$ & - & • & - & -- & + & $?$ & * & ${ }^{*}$ & \\
\hline 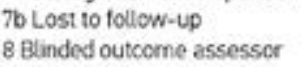 & $\stackrel{\mathrm{Na}}{+}$ & 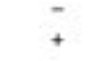 & + & $\overline{7}$ & $\stackrel{N A}{+}$ & + & $\mp$ & : & ; & $\stackrel{\text { NA }}{?}$ \\
\hline $\begin{array}{l}9 \text { Relevvance measures } \\
\text { 10a Timin assessment }\end{array}$ & : & : & : & : & : & t & $?$ & : & $\dot{+}$ & : \\
\hline 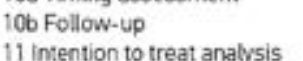 & NA & & & & NA & $=$ & 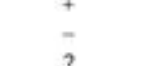 & + & + & NA \\
\hline Total & $3.5 / 11$ & $7 / 11$ & $8.5 / 11$ & $8.5 / 11$ & $6.5 / 11$ & $6 / 11$ & $4 / 11$ & $8 / 11$ & $5.5 / 11$ & $2 / 11$ \\
\hline 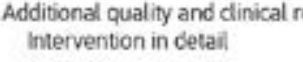 & tems. & $?$ & $?$ & $?$ & $?$ & + & $?$ & * & • & - \\
\hline $\begin{array}{l}\text { SSidide eftects } \\
\text { Sample size a priori }\end{array}$ & $=$ & : & : & : & ? & \pm & 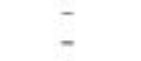 & ? & † & \pm \\
\hline Adequate statistics & & & & & & & $?$ & + & & \\
\hline
\end{tabular}

Seven hundred and ninety-one articles were identified in the Cochrane Central Register of Controlled Trials $(n=428)$, PubMed/MEDLINE $(n=$ 193). EMBASE ( $n=113)$ PsycINFO $(n=26)$ and PEDro $(n=31)$. Seven hundred and sixty articles were reiected on the basis of their title and abstract, the main reasons being duplicate identifications and study purposes different from analysing clinical aspects of $\mathrm{MT}$.

Thirty-one articles remained, of which the full-text was obtained. After reading the full-text versions of these studies, 10 articles were excluded due to the following reasons:
(1) Only one treatment:3033
Insufficient information on intervention and/or outcomes:3,34,35
(3) Orthopaedic conditions: ${ }^{7}$
Control and intervention conditions too similar:34
(5) Two references to same study dat to

Description of studies

The 21 included studies consisted of 10 randomized trials, of which six were parallel group RCTs and four were crossover studies. The data from the studies are shown in Table 2. We analysed the crossover studies as RCTs because we only extracted data from the first part of the studies, before perticinants crossed over to the control conditions, to avoid methodological problems associated with crossover study designs ${ }^{39} \mathrm{No}$ class II and III studies were identified but we retrieved eleven class IV studies (Table 3). Studies were very heterogenous in design, size, conditions studied and outcomes measured, as shown in Table 4 The methodological quality also varied as shown in Table 1 , and few were high quality: methodological quality scores ranged from 2 to 8.5 points on the AMCL: most of the higher quality randomized studies were conducted in stroke patients regarding upper limb functions, with four studies scoring equal to or higher than six poits on the AMCL In patients with CRPS (including two studies on poststroke CRPS) only two RCT5 40.41 and in patients with PLP only one randonized

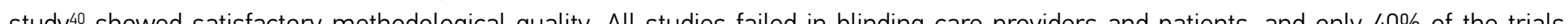
reported a repocts from the therapy and the sparse description of the treatment protocol are notable. 
Table 2. Overview of study characteristics of included randomized controlled trials

Study/score Design/parricipants

Cacchio
et al.t.

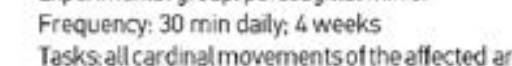

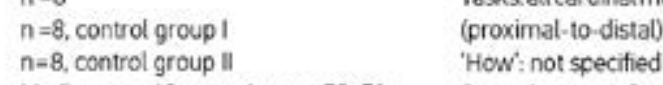

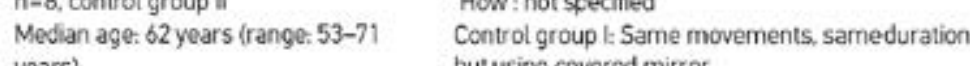

Chronic phase (median time post- Control group li: Mental practicico of the same

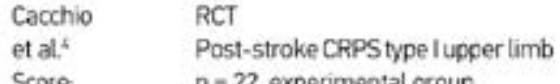
$n=22$. experimental grout
$n=20$. control group Mean age. 57.9 yed
(SD $=9.9$ years) Subacule phase $\& 6$ months 5.1 moniths: $S 0$ SD-2.5 months)

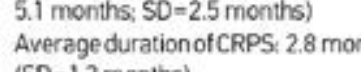
(SDD 1.3 montrs)
Experimental group: parassagittal mirror
Frequengy: first 2 weeks oncec daily for 30 min: second 2 weeks daliy 60-min sessions; 4 weeks

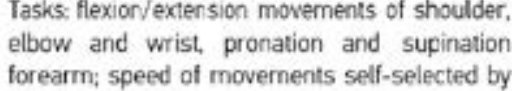
patents
How: moving only unaffected lirmb while watching

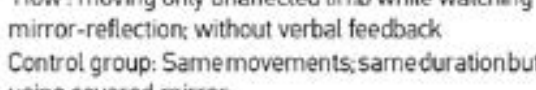
using coverere mirror
All patenents used no analgesics during study period

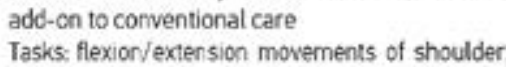

Table 2 (continued)

Results/outcom

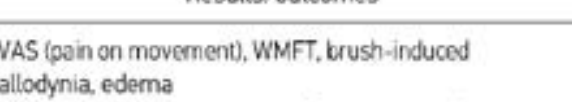

Moments: once eretest once a ther every week once

signficantrecuction otpainintensityon moverme

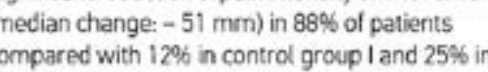

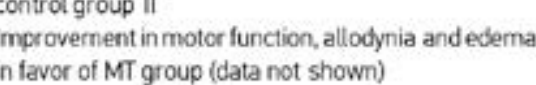

WMF, MAL,VAS (atrestiduring ghoulder flexion

woments: once pretest; once post-test 1 week at

ermination of treatment; follow-up at 6 months

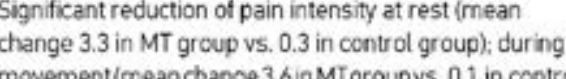

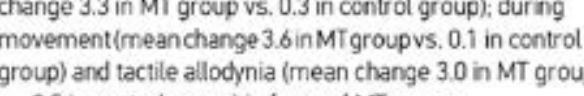

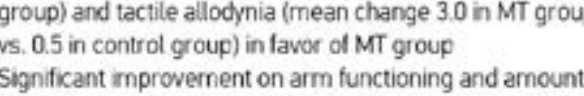

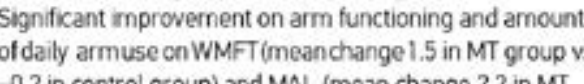
sup vs. 0.1 in control group) in taver of $M$ MT group

\begin{tabular}{|c|c|c|c|}
\hline 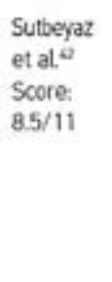 & 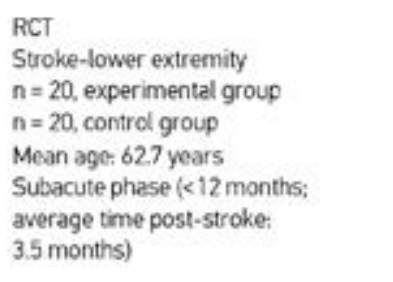 & 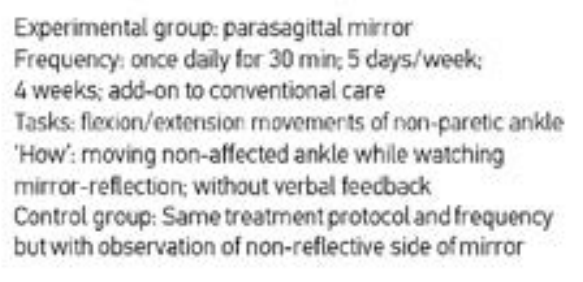 & 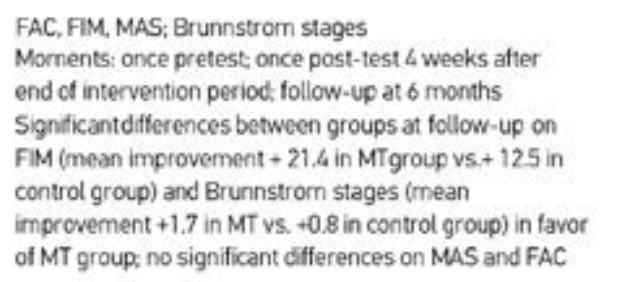 \\
\hline $\begin{array}{l}\text { Yanzzer } \\
\text { enaler } \\
\text { socore } \\
8.5 / 11\end{array}$ & 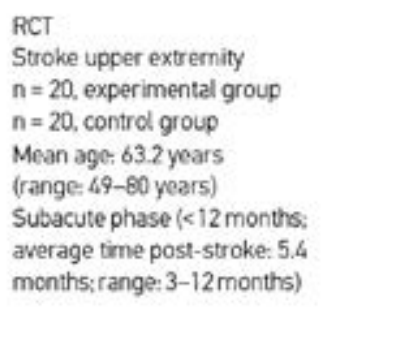 & 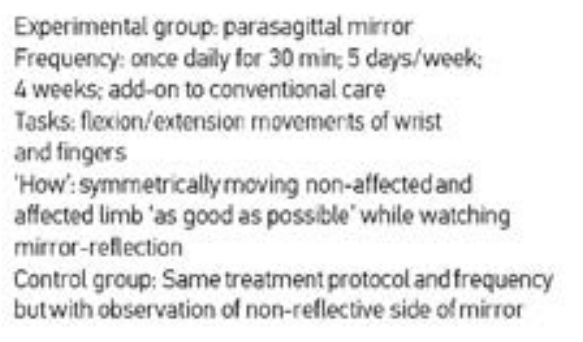 & 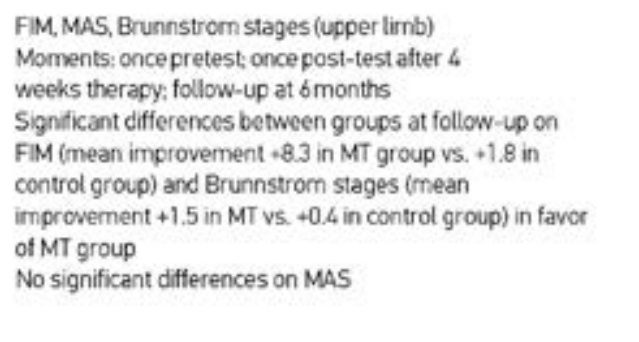 \\
\hline 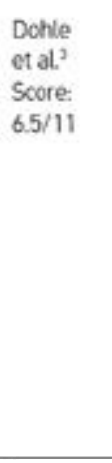 & $\begin{array}{l}\text { RCT } \\
\text { Stroke upper exremily } \\
n=18\end{array}$ & 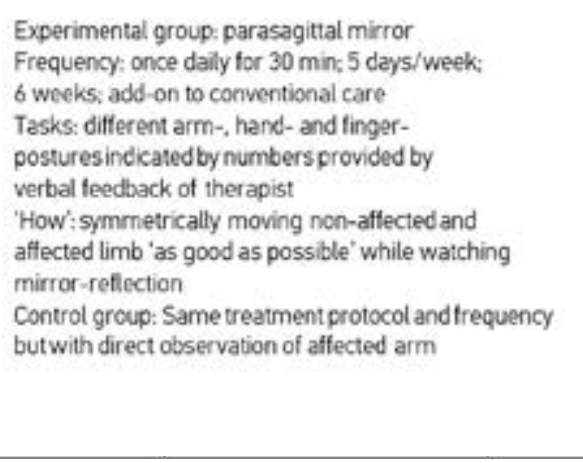 & 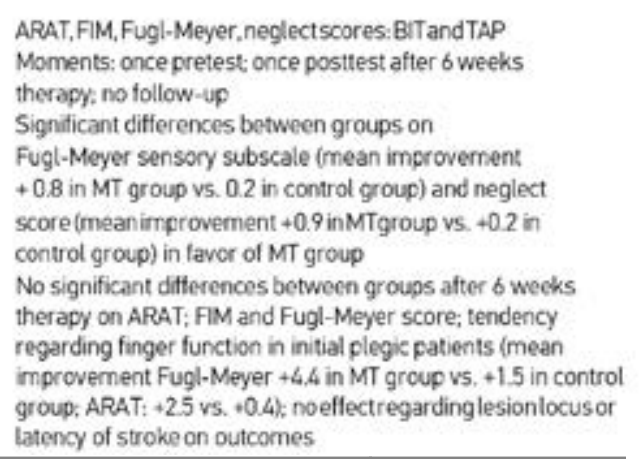 \\
\hline
\end{tabular}


Table 2 (continues)

\begin{tabular}{|c|c|c|c|}
\hline Sicyy/score & Design/participents & Interventions & Resulls/cutcomes \\
\hline 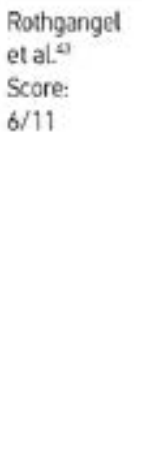 & 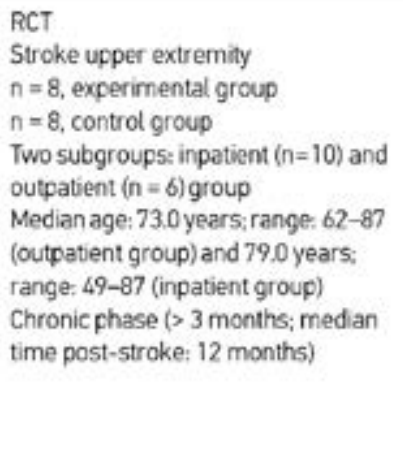 & 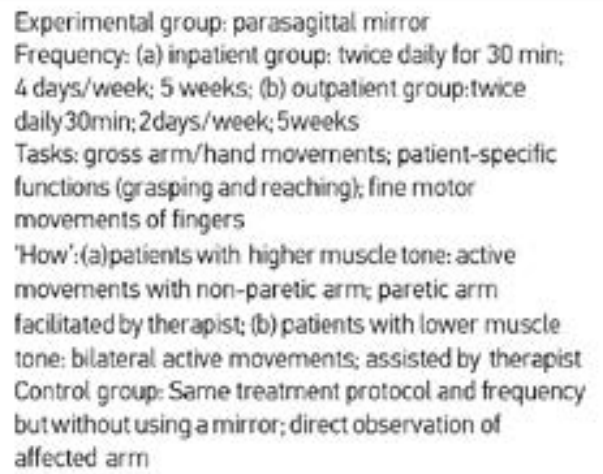 & 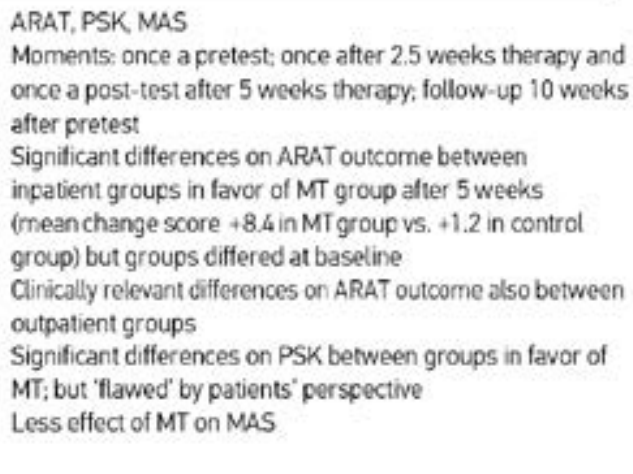 \\
\hline
\end{tabular}

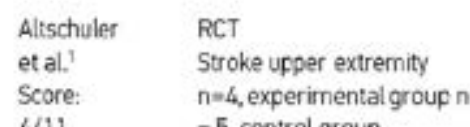

$\begin{array}{ll}\text { Score: } & \text { nes, experimental } \\ 4 / 11 & =5, \text { contror group }\end{array}$ Mean age. 59.2 years
Chronic phase $>6$ months; average tirre post-stronke.49

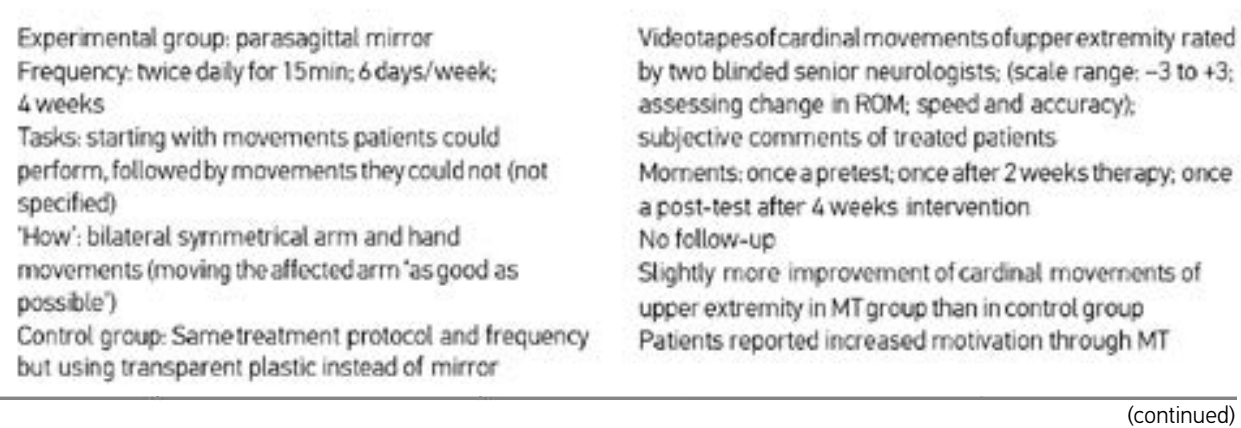

Table 2 (continued)

regional pain syndrome and phantom limb pa

Mosel
score
$8 / 11$

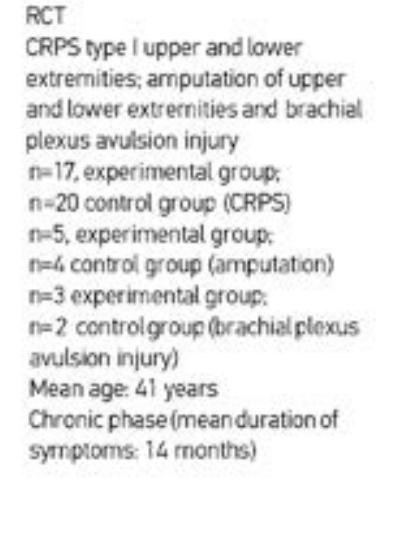

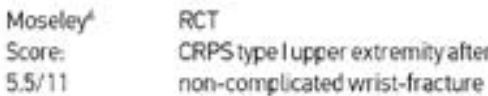

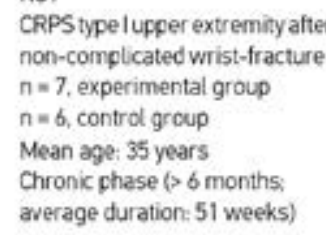

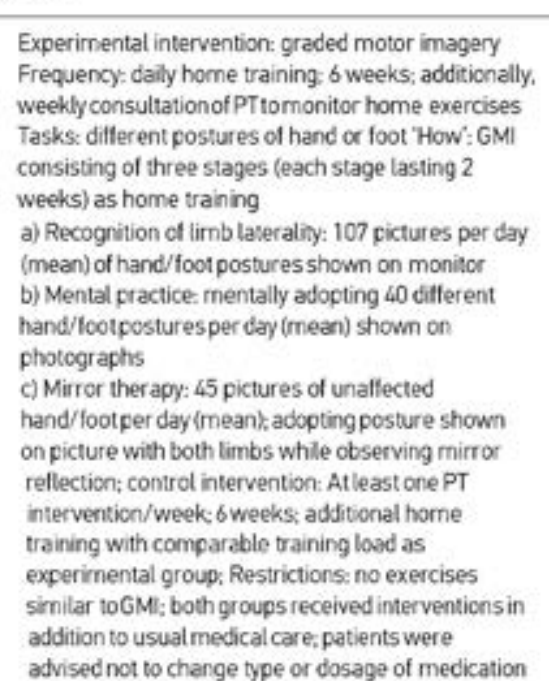

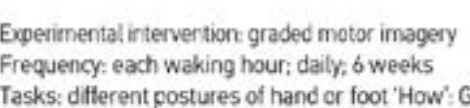

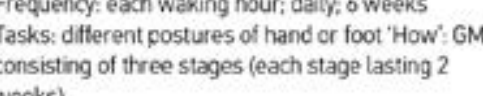

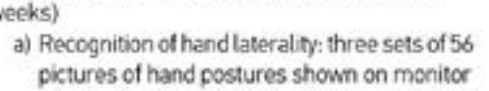

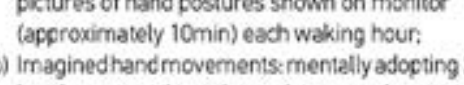

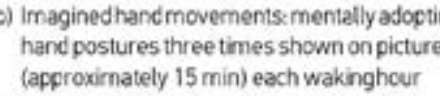
(aeproximatelly 15 min) each wakinghour

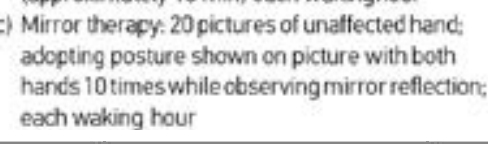

Patien-spectic tunctions on NAS; pain seventy on VAS:

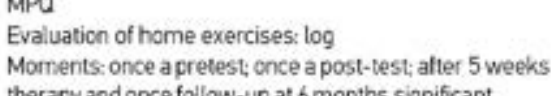

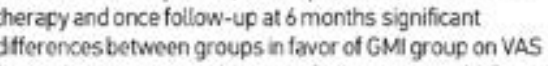

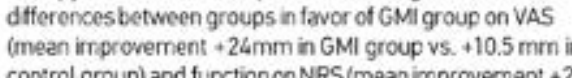

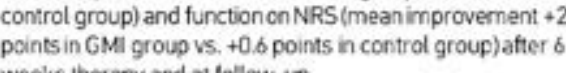

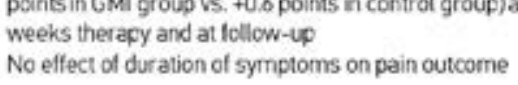

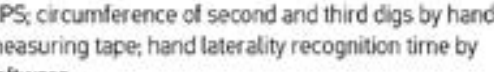

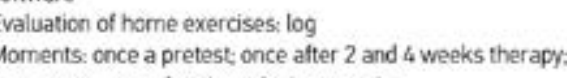

nce a poss-tes a tater 6 meets intervention

Signticant difterences between groups regarding pain

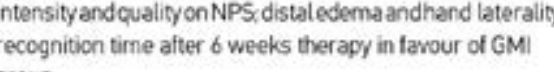

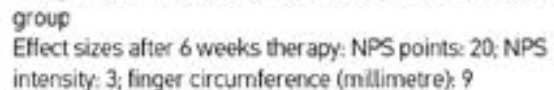


Table 2 (continues)

Complex regional pain syndrome and phantom limb pain

Study/score Design/participants Interventions Results/outcomes

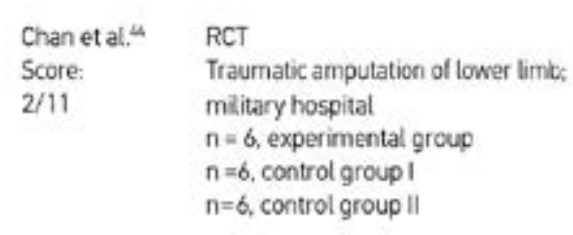

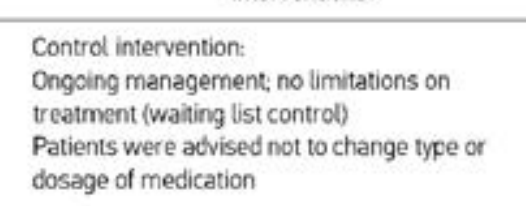

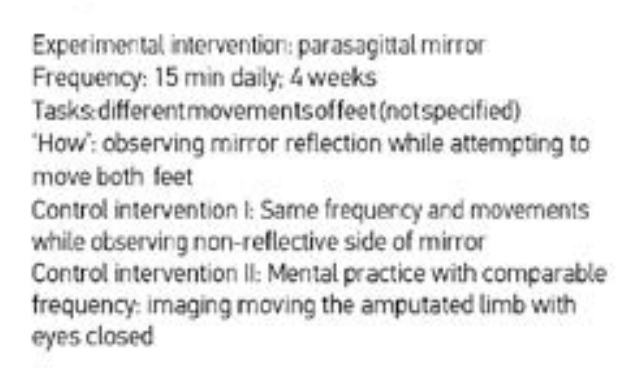

VAS: number and duration of pain episodes
Marnents: once a pretest; once atter verery week of

therepy and once a post-test atter 4 weeks therapy

follow-up

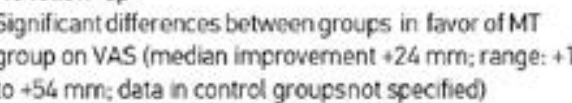
Decreased number and duration of pain exisodes

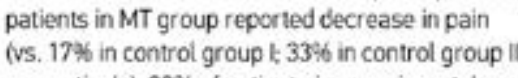
respectively) 333 o o patients in
reported acterese events (grief)

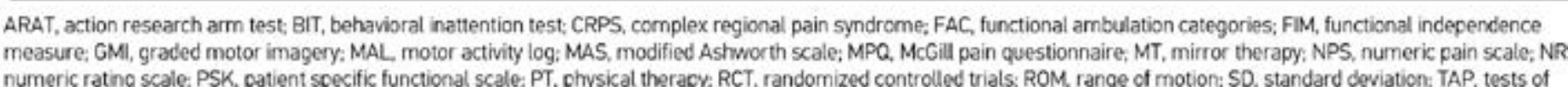

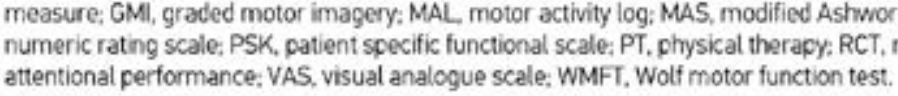

Stroke

All six randomized trials investigating the effects of MT as an additional therapy involving stroke patients showed similar results in a positive direction for arm function - Individual studies suggested positive effects on leg function ${ }^{42}$ and on sensation and neglect ${ }^{3}$ whereas two studies showed that MT reduced pain intensity and tactile allodynia in patients with CRPS type I after stroke.4,4

Three different intervention characteristics were identified: the patient was encouraged to move the affected limb 'as good as possible' 1-3 movements were only performed by the unaffected limbi, 42 or movements of the affected limb were facilitated by the therapist. ${ }^{43}$ The time between stroke and onset of the intervention varied from 26 days ${ }^{3}$ to 27 months, ' with the majority of trials including patients of no more than 12 months post-stroke. The study carried out by Dohle et al. ${ }^{3}$ suggests a correlation between the severity of paresis and amount of functional improvement by MT. Nevertheless, it was not possible to discern any firm evidence that patient characteristics or specific treatment characteristics had any infuence,

\section{Complex regional pain syndrome}

In patients with CRPS type I (including two studies on post-stroke CRPS). MT alone 4 ,41 or in combination with limb laterality recognition and mental practice, also called as 'graded motor imagery' 6,40 showed positive results in all four randomized studies It should be noted that the

In contrary to the studies of stroke patients, trials in patients with CRPS did not include active movements of the affected limb in their treatment protocols during the first weeks Instead, unilateral pain-free movements of the unaffected limb were used 4.41 or MT was preceded by other cognitive treatment strategies such as limb laterality recognition or mental practice.6, 60

Compared with the studies including stroke patients, a higher treatment frequency (several sessions per day) was used in CRPS trials. 
Table 3 Study characteristics of included class N S sudies

\begin{tabular}{|c|c|c|c|}
\hline \multicolumn{4}{|l|}{ Stroke } \\
\hline Study & Design/participants & Interventions & Results/outcomes \\
\hline $\begin{array}{l}\text { Miltuner et } \\
\text { al* }\end{array}$ & 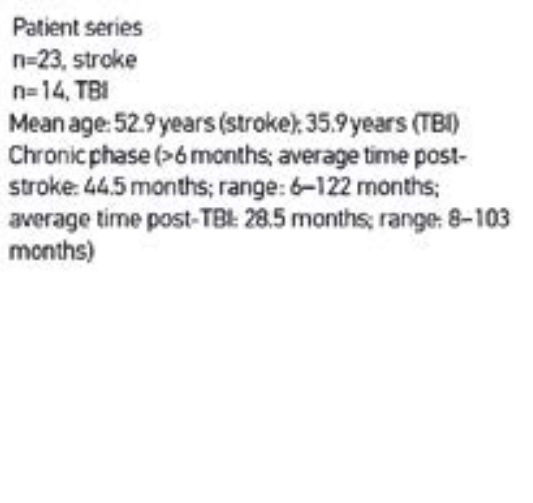 & 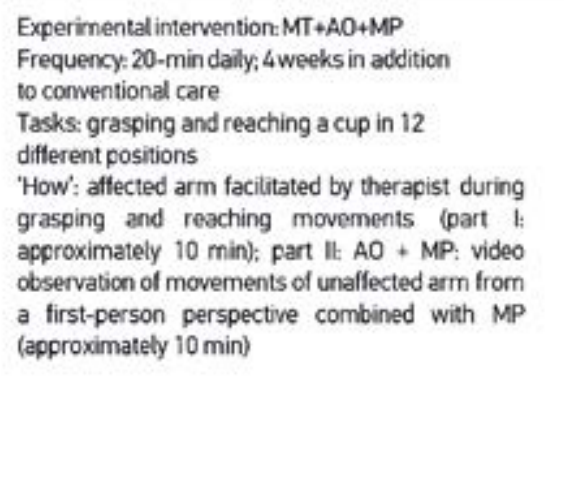 & 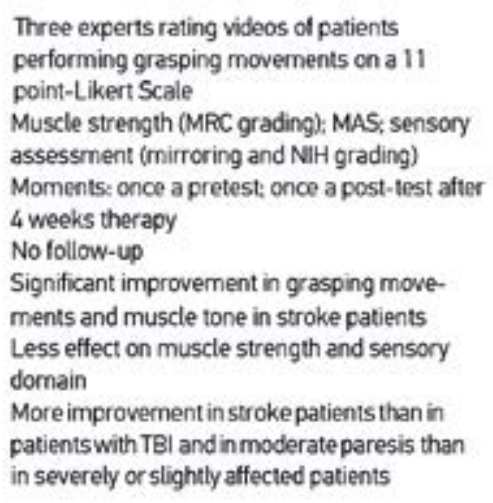 \\
\hline $\begin{array}{l}\text { Stevers and } \\
\text { Stogkhor" }\end{array}$ & 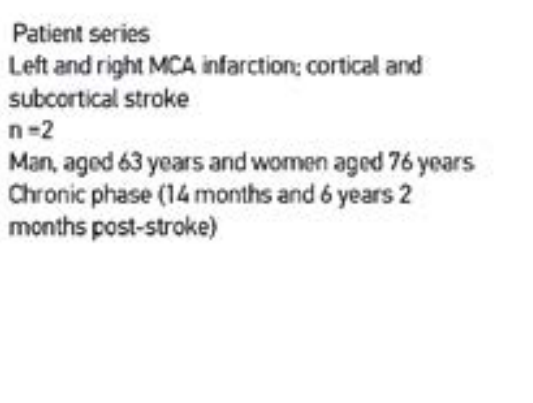 & 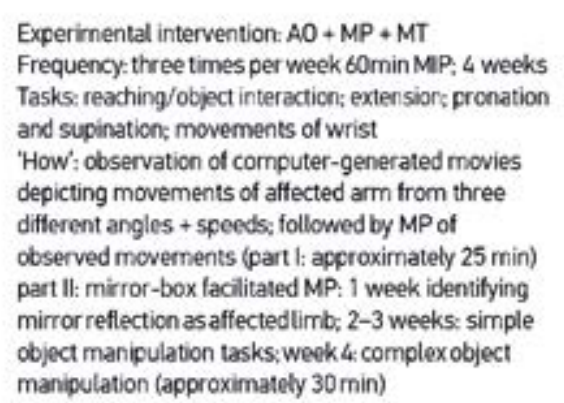 & 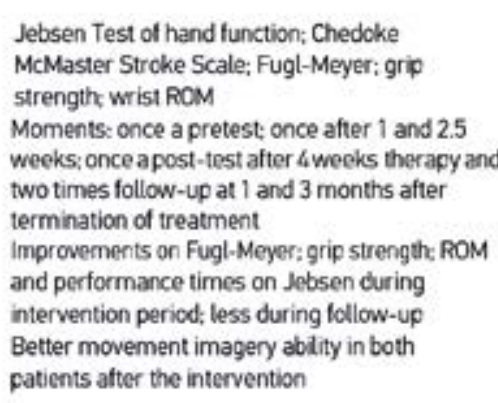 \\
\hline
\end{tabular}

Table 3 (coninued

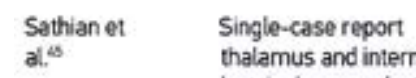

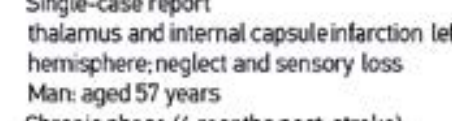 Chronic phase (s monnhs post-stroke)}

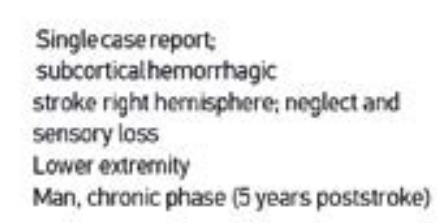

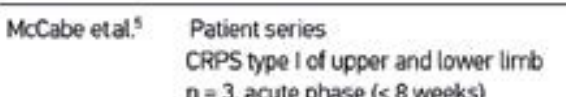

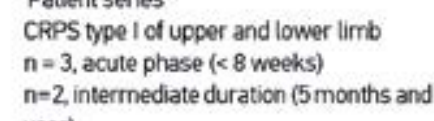

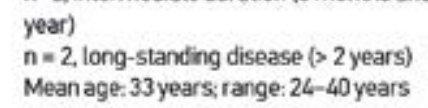

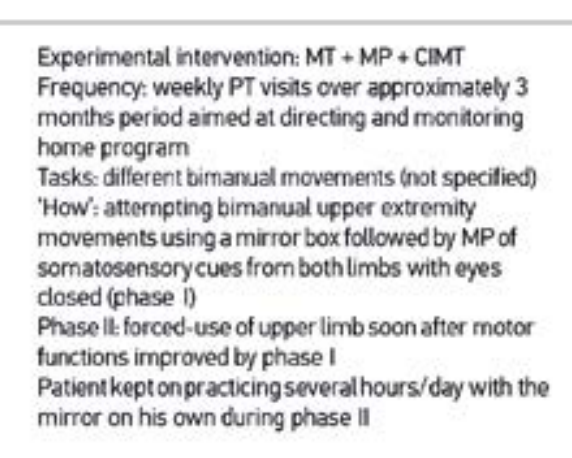

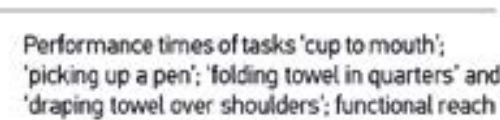

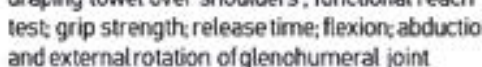

Moments: conce a pretesti once a post-tist

Unnctional arm improverement (exterended

mprovementingripstrengithrelease time and

o effect on som

Motor Function Assessment Scate, ankle ROM musclef tocce (MRC): Sensory assessment

analysisiandilomwalkingtest

Moments:
12 weeks
No ofllow-wipe

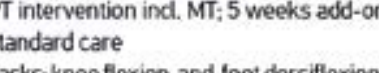

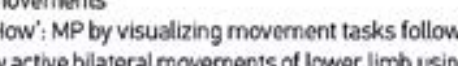

mirror; additional facilitation by thererpist A t the hen

Ditive effects on functional bibities, active

Rom, musclete toneneand-torce and sensony

\begin{tabular}{|c|c|}
\hline 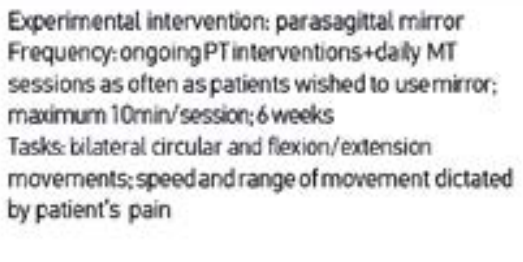 & 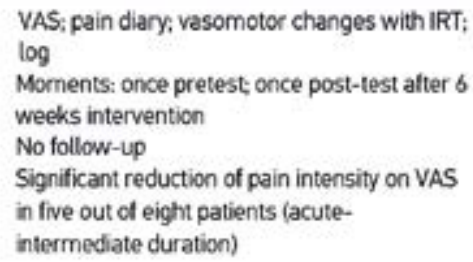 \\
\hline
\end{tabular}


Table 3 (continued)

\section{Complex regional pain syndrome and phantom limb pain}

\section{Stuofy \\ Design/participants}

Interventions

Results/curcornes

Average diseaseduration: 1 year 5 month ths
(range: 3 week-3 3 years)

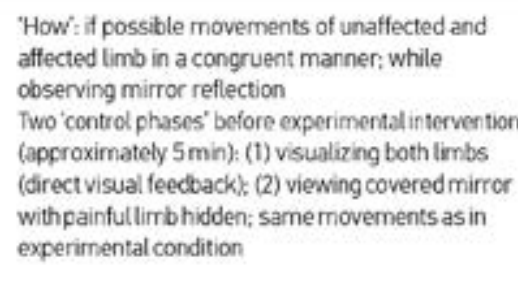

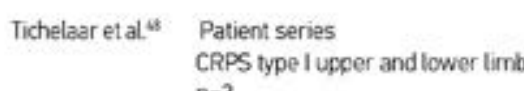

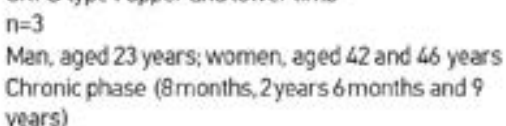

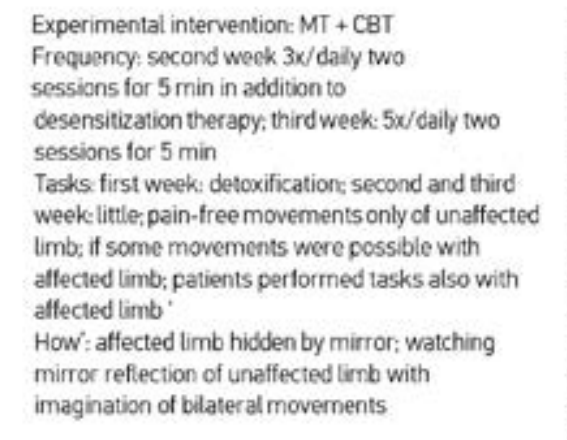

Normalization in vasomotor changes of
affiected limb

Miree out of eight patients were pain-free
sther 6 wecks therapy

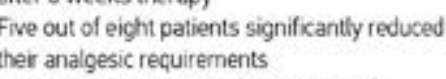

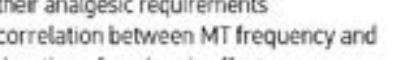
three out of feight patients (chronic phasese

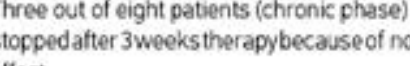

VAS (at rest and atter strength testing ard Sodniat, hand held dynamometer:

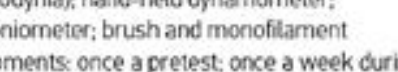

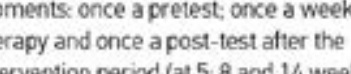
intervention period (at5. \& and 4 weeks
respoctioly)
No follow-uf

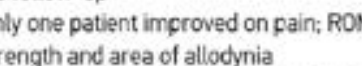

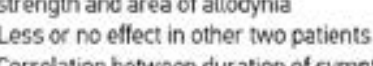

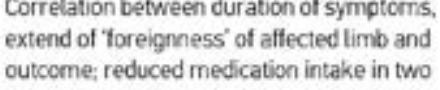

Selles et at.t.s
Pationt series
CRPS type II (causalgaia) upper limb atter

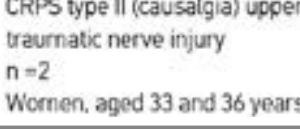

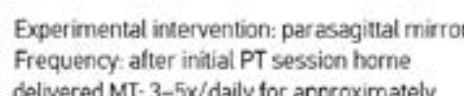
Short-term pain reliet on V VAS (patient 1).

pain relef on VAS (ratien 2)
Table 3 (continued)

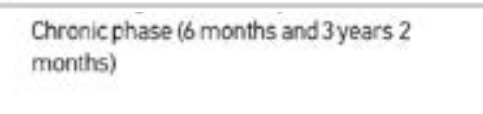

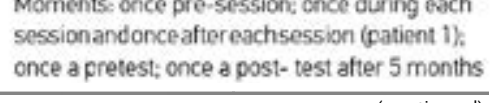

Mercier and
Sirigus

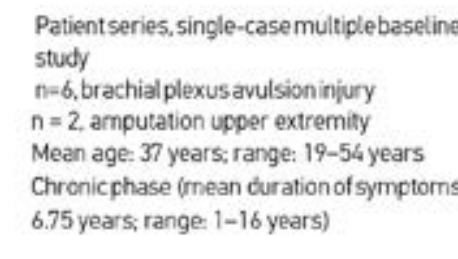

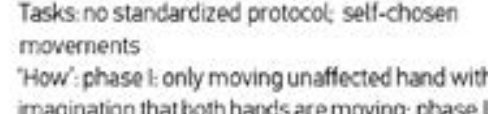

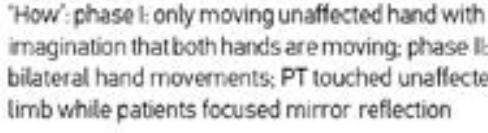

Experimental intervention: inverted image of
unaffected armina $45^{\circ}$ orented nnirror

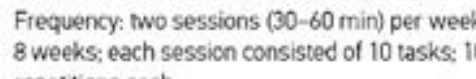

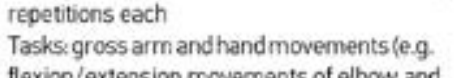

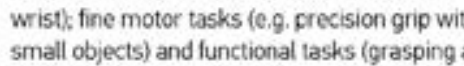

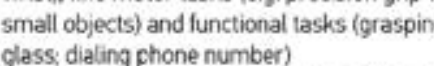

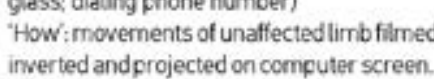

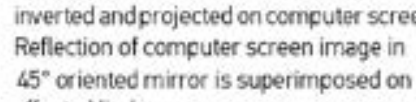

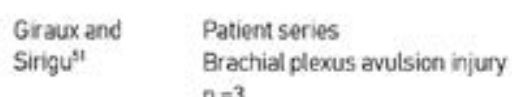
$n=3$
Men: anged 18. 20 and 41 y yars
Chronich hase

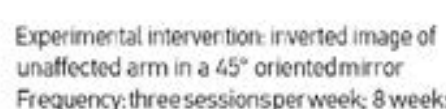

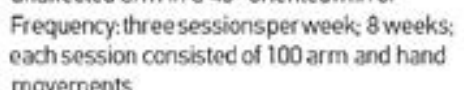

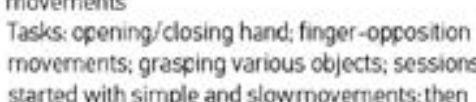

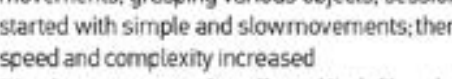

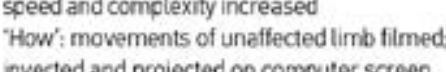
inverted and projected on computer screen

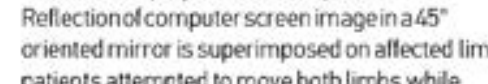

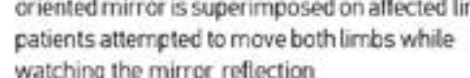

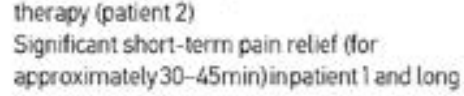

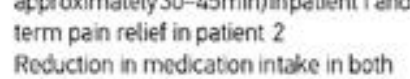

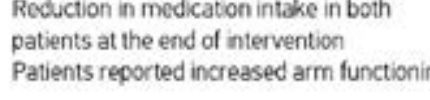

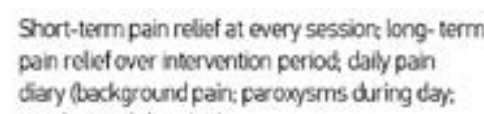

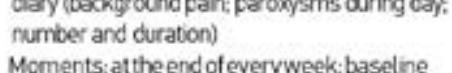

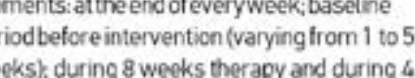

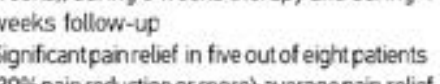

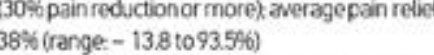
duration of syontoms No associaton between type of phantom
limb sensation and outcome

Nerage pain on VAS percentage of pain relet
WVAS: MRI1

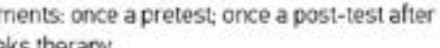

Notollow-up

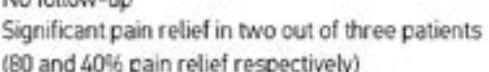
One patient showed no improvernent: MMFII

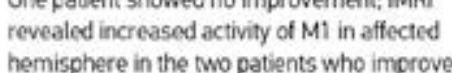

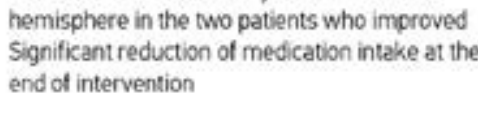


Table 3 (continued)

\section{Complex rogional pain syndrome and phantom limb pain}

Sudy Desigo/perticipants

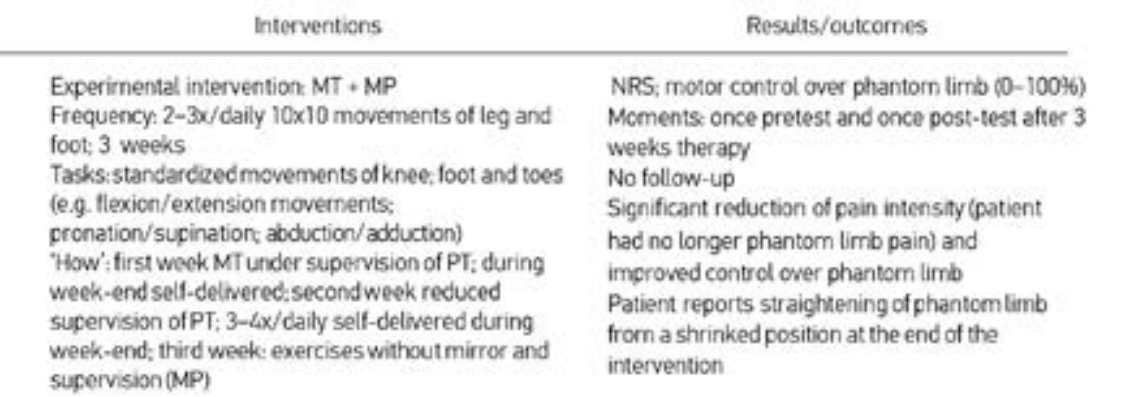

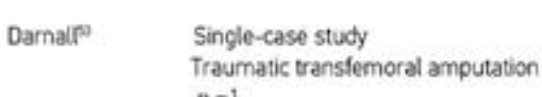

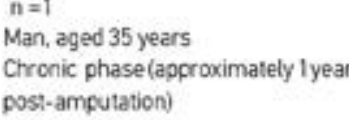

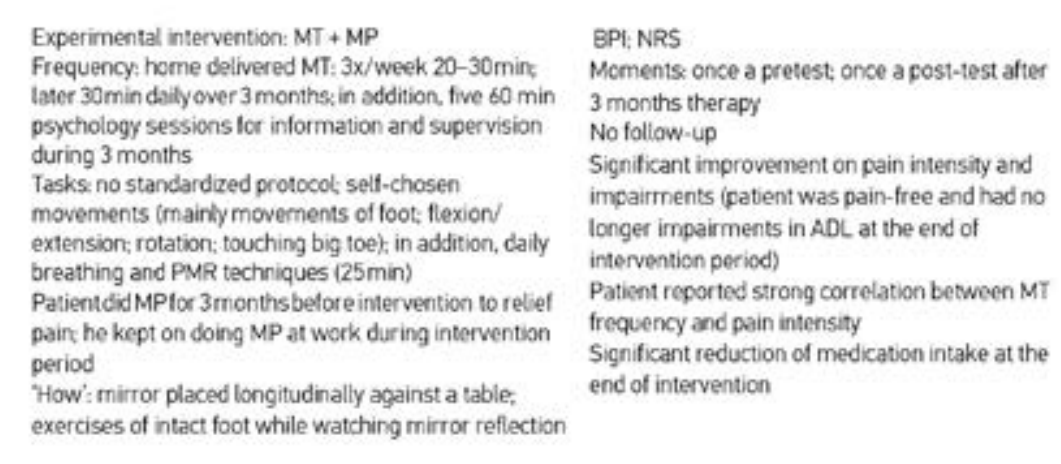

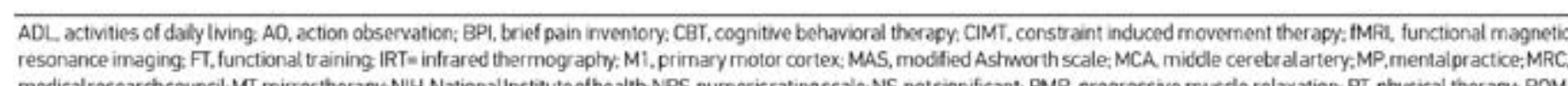

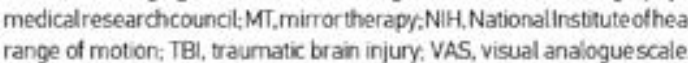

Table 4 Summary of selected class land I studies

Class s suderes

Patholog

Site Typeof intervention

Eflects $\underset{\substack{\text { Totaln } \\ \text { (eatents) }}}{\cos (0)}$

\begin{tabular}{|c|c|c|c|c|c|}
\hline 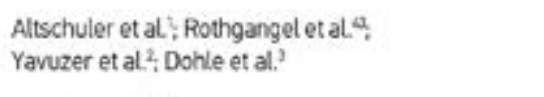 & Stroke & UE & Parassegtralmimor & $\begin{array}{l}\text { Functions, serstilly, } \\
\text { neglect }\end{array}$ & 101 \\
\hline 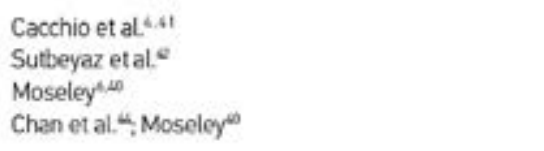 & $\begin{array}{l}\text { Post-srokek CRPS } \\
\text { Scrock } \\
\text { CPRS Sype I } \\
\text { PLP }\end{array}$ & $\begin{array}{l}\text { UE } \\
\text { LE } \\
\text { UELLE } \\
\text { UE; LE }\end{array}$ & 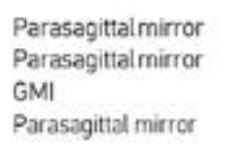 & $\begin{array}{l}\text { Pain functions } \\
\text { Functions } \\
\text { Pain functions, edema } \\
\text { Pain tunctions }\end{array}$ & $\begin{array}{l}66 \\
40 \\
50 \\
32\end{array}$ \\
\hline Class N S studies & Pathology & Site & Typeol intervention & Ellects & $\begin{array}{c}\text { Totaln n } \\
\text { (patients) }\end{array}$ \\
\hline 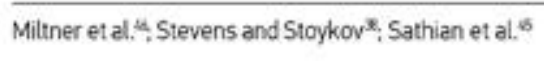 & Stroke & UE & MT+MP and $A 0+C I M T$ & Functions, muscle tone. & 26 \\
\hline Pott? & Stroke & LE & MT.MPandf & $\begin{array}{l}\text { Funcions ROM muscle } \\
\text { Fons }\end{array}$ & 1 \\
\hline 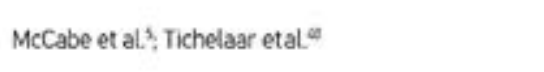 & CRPS type I & UE:LE & MT and MT+CBT & 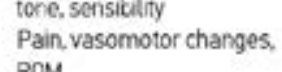 & 10 \\
\hline 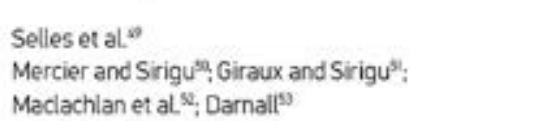 & $\begin{array}{l}\text { CRPS type II } \\
\text { PLP }\end{array}$ & $\begin{array}{l}\text { UE } \\
\text { UE;LE }\end{array}$ & $\begin{array}{l}\text { Parasaggittal mirror } \\
\text { MT and MT+MP }\end{array}$ & $\begin{array}{l}\text { PoM } \\
\text { Pain } \\
\text { Pain impairments }\end{array}$ & ${ }_{13}^{2}$ \\
\hline
\end{tabular}

作, LE L

Phantom limb pain

The two studies that investigated the effects of MT4 and graded motor imagery ${ }^{40}$ on PLP in patients following amputation of the upper or lower limb or brachial plexus avulsion, found positive results regarding patient-specific functions ${ }^{40}$ and pain intensity and number and duration of pain episodes. ${ }^{40.44}$ Unfortunately, the description of study characteristics in the publication of Chan et al..4 was sparse. 
Additional information from class IV studies

The uncontrolled studies support the findings from the class I studies. In contrary to the randomized trials in stroke patients, the intervention used in all class IV studies consisted of a combination of MT with other cognitive treatment strategies such as mental practice or action observation 15,55,46 Outcomes from CRPS trials further suggest that the degree of 'foreignness' of the affected limb as perceived by the patient and the duration of symptoms of CRPS could play an important role as a prognostic factor regarding the success of a MT intervention. 5.47

\section{DISCUSSION}

Ten randomized studies are included in this systematic review. Studies are heterogenous in design, use different measures at different times and often include small numbers of unrepresentative patients. In addition, important clinical aspects of MT interventions such as a thoto a GRADE-table was not possible, and the results could be overturned by upcoming trials; all conclusions should thereby be considered with the GRADE-approach (Grading of Recommendations Assessment Development and Evaluation).54 Because of the heterogeneity of included the recovery of lower limb functions is still low, with only one RCT'2 reporting effects. In patients with CRPS and PLP, the quality of evidence is also low.

Patient characteristics

Because of the limited evidence of included studies, no firm conclusions could be drawn regarding the important question of which patients might benefit more than others from this kind of treatment. The studies were too small and data were not provided in a way that allowed firm conclusions. But it seems reasonable that patients with insufficient attention and inford ation processing are less capable for this $k$ ind severe paresis, as proposed by Dohle et al. ${ }^{3}$, has to be further evaluated.
Teatment characteristics

Thadition, the evidence did not allow any conclusions to be drawn with regard to specific details of treatment, what may be more or less efective. As still several clinical methods are used in treating stroke and pain patients with MT interventions, future studies have to identify which treatment characteristics are more effective than others, enabling the design for clinical protocols. Remarkably, only two studies have reported on adverse effects of an MT intervention, 4,55 finding them to be clinically significant and not infrequent. In the retrospective study of Casale et al. 5529 out of 33 patients with PLP withdrew from MT treatment because of side effects such as grief, confusion or dizziness. These results show the potential adverse reactions that can be induced by the intervention and are in line with the results as that of Moseley et al., 56 who showed that motor imagry led to increased pain and swelling in patients with chronic arm pain. Similar observations were made in other studies ${ }^{57.5}$ C Consequently, given the moderate quality of evidence for beneficial effects one cannot support widespread uncritical clinical use of this technique until there is stronger evidence of benefit and evidence that it outweighs any risk or harm.

\section{Strength and weaknesses of this study}

The main strength of our study is that we focused on important clinical aspects regarding a relatively new intervention, and used systematic and explicit methods in identifying relevant trials. Furthermore, we think that we provided a comprehensive overview on the topic, adding recently published trials that have not been assessed before. This study also has some limitations. Owing to the heterogeneity of identified studies and the small number of patientsitwas impossible to give precise guidance on the right target group for MT Furthermore. conclusions about which particular method of MT in which phase of recovery might be more effective, were not possible It was not easy to define MT. because a mirror is simply one way of achieving a visuar ilusion Moreover athough it is likely that using the wearch term ' $\mathrm{n}$ de' would result in identifying all studies that used mirrors to achieve a visual illusion, it is possible that some studies were missed It is also difficult to and clinical effects. Despite these limitations, we probably identifed most of the randomized trials to give an informative overview on the clinical aspects of MT.

Conclusion

The work on MT needs to be considered in the context of any new treatment modality. Early enthusiasm attracts many researchers to experiment on small groups of selected patients, often with weak study designs and a variety of measures. This can be seen, for example, 
in the use of mental imagery and practice ${ }^{59}$ and in the application of new drugs such as cannabis extracts. ${ }^{60}$ The benefit of a relatively early systematic study, such as this, is that it may draw attention to some important points that should be considered in the design of future research. Future studies should try to identify patients who might profit more by MT than others, to guide more specific intervention through MT. Included studies did not provide sufficient information on the clinical protocols used. Therefore, detailed clinical protocols are urgently of a treatment. Future studies must systematically register adverse effects. One passibility to weigh risks and benefits could be the use of standardized assessments as proposed by Boers et a ${ }^{6.1}$ To answer these questions there is a need of multicentre studies using a smaller number of standardized and clinically relevant outcome measures that investigate the effects of MT in routine clinical settings. ${ }^{62}$ 


\section{REFERENCES}

Altschuler EL, Wisdom SB, Stone L, et al. Rehabilitation of hemiparesis after stroke with a mirror. Lancet. 1999; 353: 2035-6.

ravuzer seles R, Sezer $\mathrm{N}$, el al. Mirror therapy improves hand function in subacute stroke: a randomized controlled trial. Arch Phys Med Rehabi. 2008,89. 373-8.

Dohle C, Pullen J, Nakaten A, Kust J, Ricz Cand Karbe H. Mirror therapy promotes recovery from severe

hemiparesis. a randimized controlled trial. Neurorehabil Neural Repar. 2009; 23: 209-17.

Cacchio A, De Blasis E, Necozione S, di Orio F and Santili V. Mirror therapy for chronic complex regional

pain syndrome type 1 and stroke. N Engl J Med. 2009, 361. 634-6.

McCabe CS, Haigh RC, Ring EF, Halligan PW, Wall PD and Blake DR. A controlled pilot study of the utility of mirror visual feedback in the treatment of complex regional pain syndrome (type 1). Rheumatology (Oxford). 2003; 42: 97-101.

Moseley GL. Graded motor imagery is effective for long-standing complex regional pain syndrome: a randomised controlled trial. Pain. 2004; 108: 192-8.

7. Rosen B and Lundborg G. Training with a mirror in rehabilitation of the hand. Scand J Plast Reconstr Surg Hand Surg. 2005; 39: 104-8,

8. Gruenert-Pluess N, Hufschmid U, Santschi L and Gruenert J. Mirror Therapy in Hand Rehabilitation: A Review of the Literature, the St Gallen Protocol for Mirror Therapy and Evaluation of a Case Series of 52 Patients. Br J Hand Ther. 2008; 13: 4-9.

9. Ezendam D, Bongers RM and Jannink MJ. Systematic review of the effectiveness of mirror therapy in upper extremity function. Disabil Rehabil. 2009; 31: 2135-49.

10. Buccino G, Solodkin A and Small SL. Functions of the mirror neuron system: implications for

neurorehabilitation. Cogn Behav Neurol. 2006; 19: 55-63.

Filimon F, Nelson JD, Hagler DJ and Sereno MI. Human cortical representations for reaching: mirror neurons for execution, observation, and imagery. Neuroimage. 2007; 37: 1315-28.
12. di Pellegrino G, Fadiga L, Fogassi L, Gallese V and Rizzolatti G. Understanding motor events: a neurophysiological study. Exp Brain Res. 1992; 91: 176-80.

13. Rizzolatti G, Fadiga L, Gallese V and Fogassi L. Premotor cortex and the recognition of motor actions. Brain Res Cogn Brain Res. 1996: 3: 131-41.

14. Keysers C, Wicker B, Gazzola V, Anton JL, Fogassi L and Gallese V. A touching sight: SII/PV activation during the observation and experience of touch. Neuron. 2004; 42: 335-46.

15. Stevens JA and Stoykov ME. Using motor imagery in the rehabilitation of hemiparesis. Arch Phys Med Rehabil. 2003; 84: 1090-2.

16. Matthys K, Smits M, Van der Geest JN, et al. Mirror-induced visual illusion of hand movements: a functional magnetic resonance imaging study. Arch Phys Med Rehabil. 2009; 90: 675-81.

17. Diers M, Christmann C, Koeppe C, Ruf M and Flor H. Mirrored, imagined and executed movements differentially activate sensorimotor cortex in amputees with and without phantom limb pain. Pain. 2010; 149: 296-304.

18. Michielsen ME, Smits M, Ribbers GM, et al. The neuronal correlates of mirror therapy: an fMRI study on mirror induced visual illusions in patients with stroke. J Neurol Neurosurg Psychiatry. 2011: 82. 393-8.

19. Rothgangel AS, de Bie RA, Bastiaenen CHG, van Oostenbrugge R. Backes W and Hofman P The Role of the Mirror Neuron System in Rehabilitation with Mirror Therapy following Middle Cerebral Artery Infarction - a pilot fMRI study - 51. Annual Meeting of the German Society of Medical Informatics. Biometry and Epidemiology (GMDS), Leipzig. 2006.

20. Karnath $\mathrm{HO}$. New insights into the functions of the superior temporal cortex. Nat Rev Neurosci. 2001; 2: 568-76.

21. Karnath $\mathrm{HO}$, Ferber S and Himmelbach M. Spatial awareness is a function of the temporal not the posterior parietal lobe. Nature. 2001; 411: 950-3.

22. Allison T, Puce A and McCarthy G. Social perception from visual cues: role of the STS region. Trends Cogn

Sci. 2000; 4: 267-78.

23. Ramachandran VS and Altschuler EL. The use of visual feedback, in particular mirror visual feedback, in restoring brain function. Brain. 2009; 132: 1693-710. 
24. Seidel S, Kasprian G, Sycha T and Auff E. [Mirror therapy for phantom limb pain--a systematic review] Wien Klin Wochenschr. 2009; 121: 440-4.

25. Oxford Centre for Evidence-based Medicine. Levels of evidence. 2009.

van Tulder $\mathrm{M}$, Furlan $\mathrm{A}$, Bombardier $\mathrm{C}$ and Bouter $\mathrm{L}$. Updated method guidelines for systematic reviews in the cochrane collaboration back review group. Spine (Phila Pa 1976). 2003; 28: 1290-9.

van der Velde G, van Tulder M, Cote P. et al. The sensitivity of review results to methods used to appraise and incorporate trial quality into data synthesis. Spine. 2007; 32: 796-806.

28. Cochrane Back Review Group. Clinical relevance assessment form. 2010

29. van Tulder MW, Ostelo R, Vlaeyen JW, Linton SJ, Morley SJ and Assendelft WJ. Behavioral treatment for Spine. 2001: 26: 270-81.

30. Brodie EE, Whyte A and Niven CA. Analgesia through the looking-glass? A randomized controlled trial investigating the effect of $v$ in J Pain. 2007: 11: 428-36.

Brodie EE, Whyte A and Waller B. Increased motor control of a phantom leg in humans results from the visual feedback of a virtual leg. Neurosci Lett. 2003; 341: 167-9.

32. Ramachandran VS, Altschuler EL, Stone L, Al-Aboudi M, Schwartz E and Siva N. Can mirrors alleviate visual hemineglect? Med Hypotheses 1999: 52: 303-5.

Sumitani M. Miyauchi S. MCCabe CS, et al. Mirror visual feedback alleviates deafferentation pain. depending on qualitative aspects of the pain: a preliminary report. Rheumatology (Oxford). 2008; 47: 1038-43. Altschuler EL and $\mathrm{Hu} \mathrm{J}$. Mirror therapy in a patient with a fractured wrist and no active wrist extension. Scand J Plast Reconstr Surg Hand Surg. 2008; 42: 110-1.

Karmarkar A and Lieberman I Mirror box therapy for complex regional pain syndrome. Anaesthesia. 2006; 61: 412-3.

36. Moseley GL. Is successful rehabilitation of complex regional pain syndrome due to sustained attention to the affected limb? A randomised clinical trial. Pain. 2005; 114: 54-61.
37. Rothgangel AS, Morton A, van den Hout JWE and Beurskens AJHM. Mirror therapy in rehabilitation after stroke: Effectiveness on upper limb functioning in chronic stroke patients. Neur Rehab. 2007: 13: 271-6. Stevens JA and Stoykov ME. Simulation of bilateral movement training through mirror reflection: $a$ case report demonstrating an occupational therapy technique for hemiparesis. Top Stroke Rehabil. 2004: 11: 59-66.

39. Friedman LM, Furberg CD and DeMets DL. Fundamentals of clinical trials. 3rd ed. New York: Springer,

Moseley GL. Graded motor imagery for pathologic pain: a randomized controlled trial. Neurology. 2006; 67: 2129-34

41. Cacchio A, De Blasis E, De Blasis V, Santilli V and Spacca G. Mirror therapy in complex regional pain syndrome type 1 of the upper limb in stroke patients. Neurorehabil Neural Repair. 2009: 23: 792-9.

42. Sutbeyaz S, Yavuzer G, Sezer N and Koseoglu BF. Mirror therapy enhances lower-extremity motor recovery and motor functioning after stroke: a randomized controlled trial. Arch Phys Med Rehabil. 2007. 88: 555-9.

43. Rothgangel AS, Morton A, van den Hout JWE and Beurskens AJHM. Phantoms in the brain

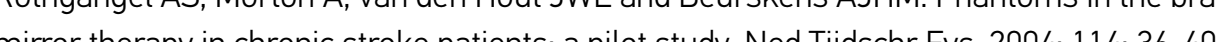

. Sathian K, Greenspan Al and Wolf SL. Doing it with mirrors: a case study of a novel approach to neurorehabilitation. Neurorehabil Neural Repair. 2000; 14: 73-6

Miltner R, Netz J and Hömberg V. Kognitive Therapie sensomotorischer Stîrungen. Krankengymnastik. 2000; 52: 954-64.

Pott C. Integration des Spiegeltrainings in ein alltagsorientiertes Therapiekonzept in der ambulanten neurologischen Rehabilitation am Beispiel eines Patienten mit zentraler Hemiparese. Krankengymnastik. 2001; 8: 1314-32. Tichelaar VYIG, Geertzen JHB, Keizer D and van Wilgen CP. Mirror box therapy added to cognitive behavioural therapy in three chronic complex regional pain syndrome type I patients: a pilot study. In $J$ Rehab Res. 2007: 30: 181-8, 
49. Selles RW, Schreuders TA and Stam HJ. Mirror therapy in patients with causalgia (complex regional pain syndrome type Il) following peripheral nerve injury: two cases. J Rehabil Med. 2008; 40: 312-4.

Mercier $C$ and Sirigu A. Training with virtual visual feedback to alleviate phantom limb pain. Neurorehabil Neural Repair. 2009; 23: 587-94.

51. Giraux P and Sirigu A. Illusory movements of the paralyzed limb restore motor cortex activity. Neuroimage. 2003; 20 Suppl 1: S107-S1

52. MacLachlan M, McDonald D and Waloch J. Mirror treatment of lower limb phantom pain: a case study Disabil Rehabil. 2004; 26: $901-4$

Darnall BD. Self-delivered home-based mirror therapy for lower limb phantom pain. Am J Phys Med Rehabil. 2009; 88: 78-81.

54. Guyatt GH, Oxman AD, Vist GE, et al. GRADE: an emerging consensus on rating quality of evidence and strength of recommendations. BMJ. 2008; 336: 924-6.

Casale R, Damiani C and Rosati V. Mirror Therapy in the Rehabilitation of Lower-Limb Amputation: Are

56. There Any Contraindications? Am J Phys Med Rehabil. 2009; 88: 837-42. hurts: the effect of motor imagery on pain and swelling in people with chronic arm pain. Arthritis Rheum. 2008; 59: 623-31.

57. Fink GR, Marshall JC, Halligan PW, et al. The neural consequences of conflict between intention and the senses. Brain. 1999; 122 ( Pt 3): 497-512

58. McCabe CS, Haigh RC, Halligan PW and Blake DR. Simulating sensory-motor incongruence in healthy volunteers: Implications for a cortical model of pain. Rheumatology (Oxford). 2005; 44: 509-16. Braun SM, Beurskens AJ, Borm PJ, Schack Tand Wade DT. The effects of mental praclice in stroke

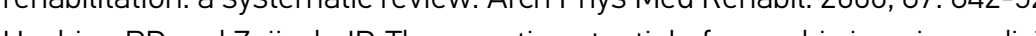

medicine. Br J Anaesth. 2008; 101: 59-68. Boers M, Brooks P, Fries JF, Simon LS, Strand V and Tugwell P. A first step to assess harm and benefit in 2009; 8: 741-54.
(Citeria for positive scoring on additional quality items (see also ${ }^{27}$ ).

(1) Calculation of sample size a priori: for a positive scoring the authors of the study have to describe the procedure of sample size calculation and present the calculated numbers of participants.

(2) Intervention described in detail: the review author judges whether the intervention was described in detail to allow replication of the intervention

(3) Side effects assessed: if the authors of the study described additional observed effects regarding the (i) positive.

(4) Adequate statistics used: the review author judges whether appropriate statistical methods were used with regard to the outcome measurements and number of groups and patients studied. 


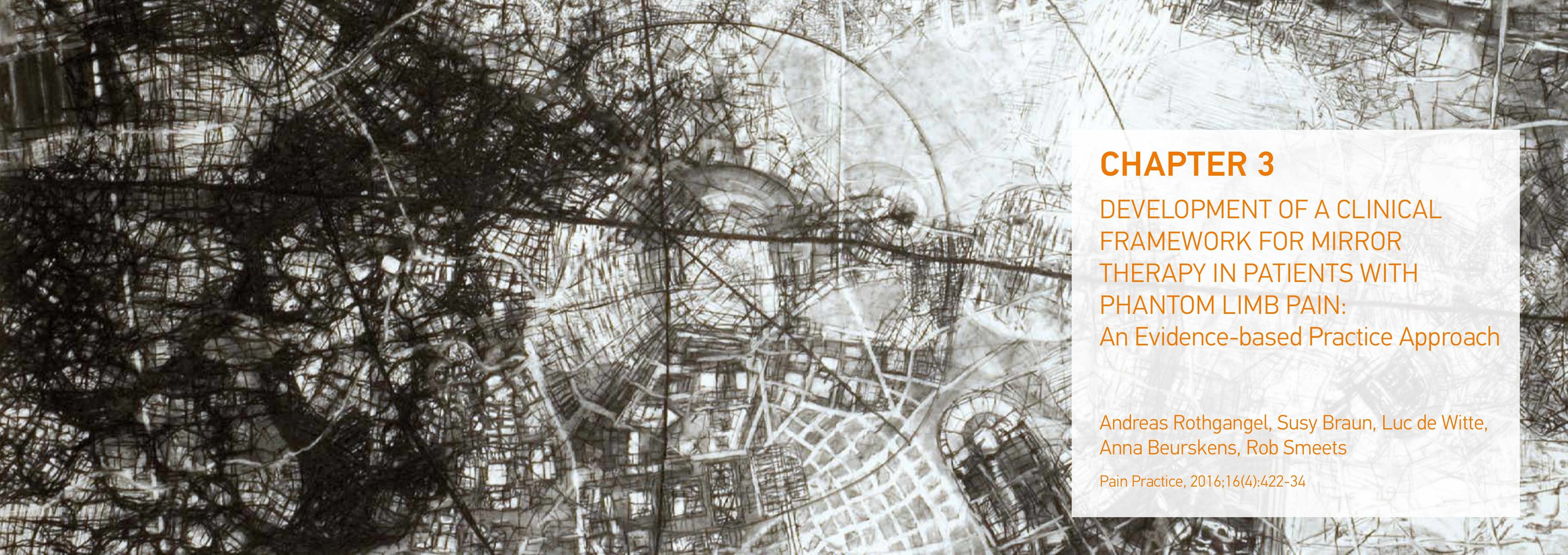


ABSTRACT

OBJECTIVE

To describe the development and content of a clinical framework for mirror therapy (MT) in patients with phantom limb pain (PLP) following

METHODS

Based on an a priori formulated theoretical model, 3 sources of data collection were used to develop the clinical framework. First, a review of the literature took place on important clinical aspects and the evidence on the effectiveness of MT in patients with phantom review of the literature took place on important clinical aspects and the evidence on the effectiveness of MT in patients with phantom
limb pain. In addition, questionnaires and semi-structured interviews were used to analyze clinical experiences and preferences of physical and occupational therapists and patients suffering from PLP regarding the application of MT. All data were finally clustered into main and and occupational therapists and patients suffering from PLP regarding the apdicat
subcategories and were used to complement and refine the theoretical model.

\section{RESULTS}

For every main category of the a priori formulated theoretical model, several subcategories emerged from the literature search, patient, and therapist interviews. Based on these categories, we developed a clinical flowchart that incorporates the main and subcategories in a logical way according to the phases in methodical intervention defined by the Royal Dutch Society for Physical hinera. In addition, we developed a

\section{CONCLUSIONS}

In this study, a structured clinical framework for the application of MT in patients with PLP was developed. This framework is currently being tested for its effectiveness in a multicenter randomized controlled trial. 


\section{INTRODUCTION}

One of the most important complaints of patients following amputation is the existence of phantom limb pain (PLP), which is perceived in the missing Despite the high number of PLP, there is curently no standard effective treatment? Treatment of PLP mainly consists of pain medication despite potential side effects, ${ }^{10}$ high costs, "1 and only low quality of evidence regarding its long-term efficacy 12

Reorganization of the somatosensory $y^{1,14}$ and motor cortex $x^{15.16}$ has been proposed to contribute to PLP. It was shown that the invasion of areas neighboring the representation of the amputated limb positively correlates with the intensity of PLP. ${ }^{17}$ In this context alternative, nonpharmacological interventions such as mirror therapy (MT) are gaining increased attention in the treatment of PLP18-20 During MT, the patient sits in front of a mirror that is oriented parallel to the patients' midline and consequently blocks the view of the amputated limb This reduce phantom limb pain ${ }^{2}$

In a recent systematic review 22 it has been reported that despite the potential merits of $M T$, the quality of evidence in patients with PLP is still low and a detailed description of how to deliver MT is missing In addition interventions do not seem to be comparable, becouse dat on important clinical aspects of MT, such as patient and intervention characteristics, are scarce. With regard to the application of MT in patients with PLP a variety of clinical methods exists, ranging from graded motor imagery ${ }^{20}$ a combination of MT and mental practice 23244 to solely using MT $18.25 \mathrm{In}$ most studies, only motor exercises are used, even though exercises using sensory stimulation seem to be equally important ${ }^{26}$ Taking together many varitions in applying MT exist, whereas detailed information and a standardized evidence based treatment protocol implementation of MT in clinical care.

Aim

The aim of this article was to describe the development and content of a clinical framework for MT in patients with PLP following amputation that is based on the best available evidence, patient preferences, and clinical expertise of physical and occupational therapists.

\section{METHODS}

Three sources of data collection were used to develop the clinical framework corresponding to the evidence-based practice approach. ${ }^{27}$ We reviewed the literature on important clinical aspects regarding MT and the evidence on the effectiveness in patients with PLP. In addition. we used questionnaires and semi-structured interviews with patients suffering from PLP who had experience in performing MT as well as physical and occupational therapists regarding their experiences and preferences regarding the application of $\mathrm{MT}$.

\section{Theoretical Model}

As a starting point, we defined a priori the theoretical model that should guide the development of the clinical framework. This theoretical model represents the phases in methodical intervention defined by the Royal Dutch Society for Physical Therapy $y^{28}$ including informing the patient, history taking physical examination, diagnosis, and indication for treatment, treatment (plan) and evaluation. These phases reflect the steps physical therapists take during the process of clinical reasoning.

In addition, we wanted to collect data on clinically relevant aspects such as facilitators, barriers, and effects of the treatment and general requirements such as exercise materials, frequency of therapy, or duration of sessions. Finally, we clustered the topics mentioned above to build a theoretical model that consists of 6 main categories: general requirements, history taking. physical examination and diagnosis.

For each category of this theoretical model, we tried to provide detailed information based on the best available evidence, patient preferences, and clinical experiences of physical and occupational therapists. 


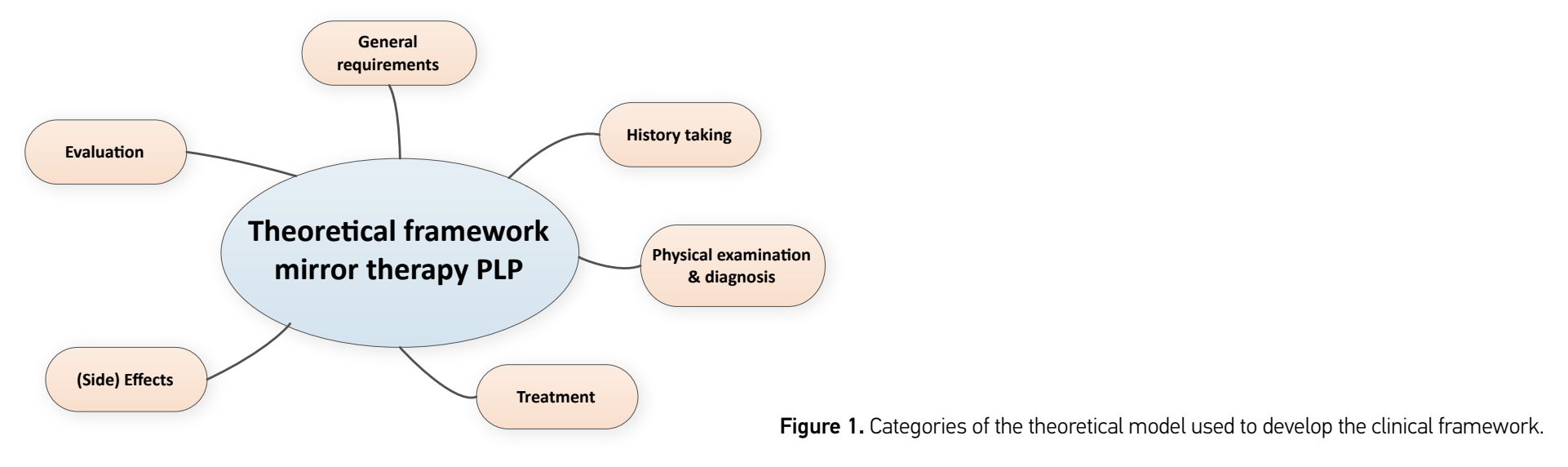

\section{Best Available Evidence}

In the following, we describe the criteria used to consider literature for this study.

Types of Studies. We included all available literature in English, German, French, and Dutch language that provided relevant information of MT in adult patients with PLP with regard to the categories of our theoretical model.

Types of Participants. All studies that addressed adult patients (aged $>18$ years) with PLP following amputation were included. No restrictions were made regarding the etiology, localization, or level of amputation.

Types of Interventions. We defined MT as the use of a mirror reflection of unaffected limb movements superimposed on the affected limb. Other similar techniques such as immersive virtual reality and studies that investigated the neurophysiological background of MT only were excluded. MT had to be provided as the only intervention or in combination with other types of treatment strategies.

Search Strategy. A computer-supported literature search from August 2010 through June 2014 was performed to update our systematic review on the clinical aspects of MTI2 using the following databases: Cochrane Central Register of Controlled Trials, PubMed/MEDLINE, CINAHL, EMBASE, PEDro, and German database DIMDI. The search strategy that was used for the databases PubMed and Cochrane served as the main protocol and was then modified for searching other databases. The following keywords were used: mirror therapy, mirror visual feedback, imagery (Psychotherapy), feedback/psychological, physical therapy, occupational therapy, amputation, amputees, phantom limb, and phantom pain. In addition, we screened reference lists and searched for publications of investigators of identified articles to retrieve additional studies. The detailed search strategy for each database is available on request from the first author (AR)

Data Collection and Analysis. Relevant data from the retrieved literature with respect to the a priori formulated theoretical model were extracted systematically using a standardized extraction form and were used to complement and specity the main categories of the theoretical model.

\section{Analysis of Clinical Expertise of Therapists and Patient Preferences}

Questionnaire. Based on our theoretical model, we developed a structured questionnaire for patients and therapists covering mainly openended questions on the following categories:

$$
\begin{aligned}
& \text { Characteristics of patient/therapist (eg, number of patients treated with MT so far, date, side, and level } \\
& \text { of amputation) } \\
& \text { Relevant aspects of MT according to theoretical model (eg, general requirements, history taking, } \\
& \text { content, and sequence of exercises) }
\end{aligned}
$$

Further examples of the questions used in the questionnaire are given in Table 1. In the therapist questionnaire, we also included a case was performed to check whether we had identified the most important aspects through the literature search.

Semi-structured Interviews. The questionnaire was checked on integrity and comprehensibility by 5 therapists and 1 patient representative. the interviews took place re 
guideline for the semi-structured interviews that were conducted by the principal investigator (AR) and were used to get in-depth information on the different categories.

Recruitment of Therapists. The principal investigator recruited German physical and occupational therapists by e-mail or phone via existing networks (eg, www.spiegeltherapie.com) using convenience sampling. At the same time, we also tried to achieve a wide range of variation in therapist characteristics (eg, profession, age, experience, work setting) to ensure rich data collection. The professionals needed to have sufficient experience in using MT for patients with PLP; "sufficient" was defined as having treated at least 3 patients during the past 12 months.

Recruitment of Patients. Patients were recruited through the treating therapists, who participated in the interviews by mail or personal communication. Furthermore, the principal investigator contacted orthopedic technicians, patient support groups and placed onle range of variation in patient characteristics (eg, age, gender, reason for amputation) to ensure rich data collection

Patients had to fulfill the following selection criteria:

- Adult patient with unilateral amputation of the lower limb.

- Sufficient experience using MT; "sufficient" was defined as a minimum of 3 sessions during the last year.

Sufficint cognitive and linguistic capaities to

questionnaire; this was based on a clinical judgment of recruiting therapists.

Patients with severe comorbidity (eg, stroke), visual constraints, or pain in the intact limb were excluded because this could prevent active engagement in the MT treatment. We recruited new patients and therapists until saturation of the data was achieved.

\section{Data Collectio}

Questionnaire. The data from the questionnaires with respect to patient and therapist characteristics were extracted and displayed in frequency table. Data regarding the clinical aspects of MT were extracted and used together with the data from the interviews to complement and refine the categories of the theoretical model.

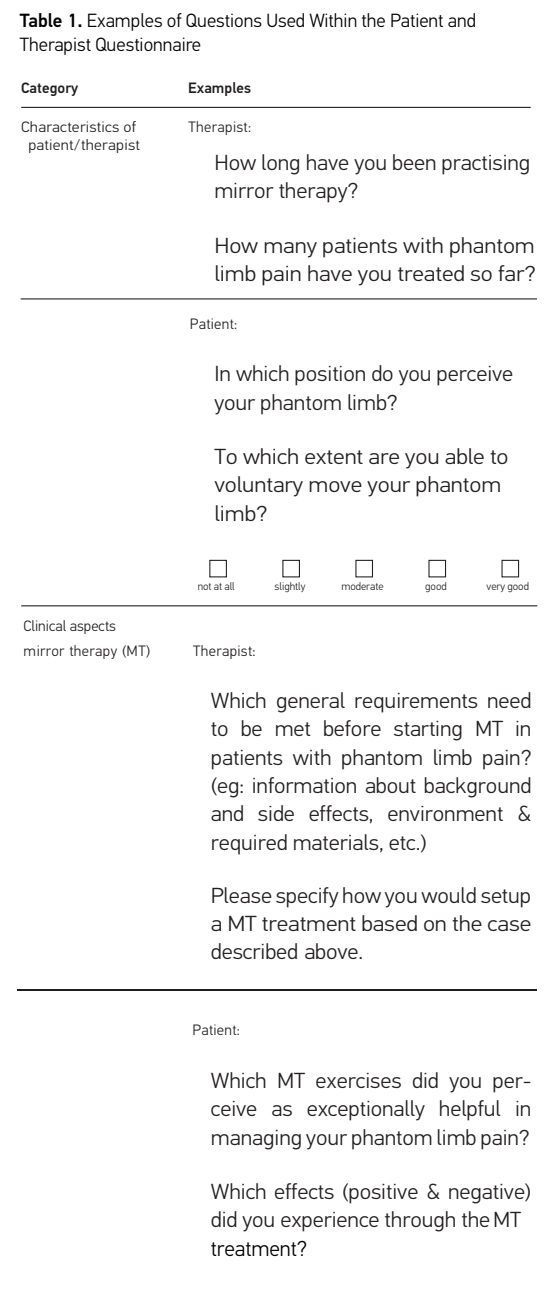

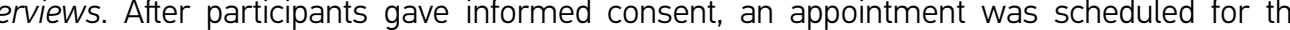
interview. All individual semi-structured interviews took place in a quiet room at patients' home or at the professional's clinic respectively, and lasted approximately 1 hour. The interviews were digitally audio recorded and subsequently transcribed using the 4 software (audiotranskription.de, Marburg, Germany). Additional field notes were made after every interview by the principal investigator (AR), describing the context of the interview.

\section{Data Analysis}

Only information with respect to our theoretical model was transcribed in German language by the principal investigator. All interview data were analyzed by directed content analysis ${ }^{29}$ The initial coding scheme was based on the a priori formulated theoretical model This scheme was used to analyze the interviews and was extended through analysis of the data. All data were summarized in a table and were subsequently sent to the interviewee who was asked to check the data on completeness and correctness (member check). The interviewee then replied the approved summary of data. Another researcher (SB) independently transcribed a sample of 3 patient and 3 therapist interviews and discussed the results with the principal investigator $A$ consensus method was used to resolve disagreements with respect to the data analysis. All data from the literature search, questionnaires, and semi-structured interviews were finally clustered into main and subcategories and were used to complement and refine the theoretical model. Finally. the mat and subcategories of the clinical framework were visually displayed using mind maps (Omnigrafle, OmniGroup), and quotes of patients were translated into English to illustrate the results. 


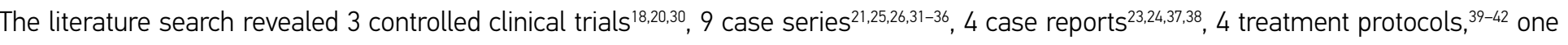
narrative review, ${ }^{43}$ and one Delphi study. ${ }^{44}$

No additional controlled clinical trials in patients with PLP were found since the publication of our systematic review.22 Data that could be extracted from existing controlled clinical trials/820,30 were sparse and mainly contained information regarding selection criteria used to ants, basic information on exercises and assessments used to evaluate effects of the in

However, the identified case studies ${ }^{21,23-26,31-38}$ provided additional information mainly on the categories history taking and content of the treatment. Three studies ${ }^{21,26,31}$ highlighted the importance of establishing and assessing the vividness of the mirror illusion (defined as the feeling that the mirror image is part of one's body), as this seems to be correlated with the effects of the training. ${ }^{2}$

Two studies $2^{2,31}$ performed a detailed interview on additional aspects regarding the phantom limb beside questions concerning PLP. These aspects include among others the usual posture and length of the phantom limb and the ability of the patient to voluntary move the phantom. In the study of Mercier and Sirigu, ${ }^{31}$ the natural position of the phantom limb was used as starting point for the motor exercises and the difficulty level of the movements was adjusted to the capacity of the phantom limb. Regarding the content of the exercises, most studies used (a) the mirror image before starting motor exercises.

Only one study' additionally used more complex functional movements with materials (eg, grasping objects). In 3 studies, ${ }^{23252.6}$ no structured exercise program was provided and patients were free to choose exercises on their own. One study's pointed out the relevance of tactile stimulation that could have additional effects above motor exercises alone. The majority of studies facilitated unsupervised training of patients as soon as possible using logs to specify exercises and to monitor frequency and quality of the training.

The 4 clinical protocol $\mathrm{s}^{39-42}$ contributed extensive information to the different categories of the theoretical model. Only one protocoliti specifically addressed patients with PLP. Two protocols ${ }^{3,4.2}$ were applied in a mixed pain population and one protocol ${ }^{60}$ mainly focused on patients with complex regional pain syndrome (CRPS) but also provided some basic information on PLP.

All studies emphasized that patients must sufficiently be instructed about the background, working mechanism, and potential side effects of the intervention. In addition, a variety of selection criteria to choose eligible patients such as sufficient cognitive abilities, trunk control psychological capacities, and a pain-free, intact limb were mentioned. With respect to the intact limb, all protocols agreed that visual marks such as jewelry, tattoos, or scars should be removed or covered to facilitate embodiment of the mirror image. Two protocols $4 \mathrm{~s}^{4,12}$ recommended

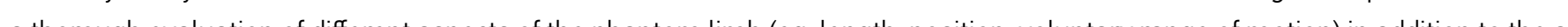

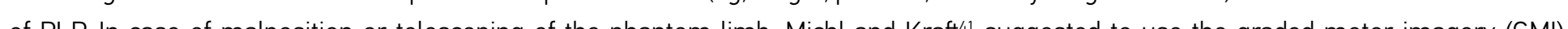
approach ${ }^{20.45}$ instead of solely using MT. Two protocols $s^{60.41}$ emphasized that the mirror illusion should be established first before starting motor exercises. The latter were performed with he unaffected limb first and as soon as patients were able to pefform pain-free movements also with the phantom limb, bilateral movements were facilitated.

With regard to the content of MT exercises, 4 different categories were identified in the different protocols.

Observation of different postures in the mirror without movement. ${ }^{39-41}$

Simple motor exercises without using objects. 39-22

Sensory exercises using different textures ${ }^{39-42}$

Complex motor exercises using objects. .11,42 $^{4}$

Mental practice and limb laterality recognition training are seen as optional additional components in the treatment program for some patients. $40-122$ In the protocol of McCabe, ${ }^{40}$ imagined movements are performed before the treatment with MT is started, to give insight into the
motor planning pathways. In another protocol, ${ }^{42}$ imagined movements of the phantom limb are preceded by mental visualization of different joints of the intact and phantom limb ("body scan"). In the same protocol, the MT treatment was divided into an evaluation and a training phase. Within the evaluation phase, which comprises 4 sessions, the therapist checks the eligibility of the patient for MT using the exercises categories described above. Eligible patients will then be trained using a tailored exercise program within the following phase consisting of up to 10 sessions. The same treatment approach was described in the narrative review by Schwarzer et al.o 
In all protocols, an individualized home program was facilitated as soon as possible and monitored using logs. Two protocols 39,40 pointed out that the treatment should be stopped in case patients do not longer perceive any henefit, symptoms have resolved, or side effects (eg nausea, dizziness, emotional reactions) are too strong.

We identified one Delphi study tht that assessed the experience of several international experts on the modalities and side effects regarding MT in patients with PLP. In this study, 11 international experts reached full consensus that sufficient education, an individual setup, face-toface guidance, and thorough monitoring and re-assessment of MT are important clinical aspects of the intervention. In addition, 5 different treatment plans were identified through the expert interviews:

1. Remote MT (instruction through leaflet and DVD, remote follow-up).

2. Intense MT (one lengthy session up to 3 hours in length)

3. Graded motor imagery. 20.45

4. Structured and supervised MT.

5. Prerecorded MT (prerecorded movements of intact limb projected onto a mirror).

In addition to the side effects mentioned in the clinical protocols, 6 of 11 experts also reported that an increase in PLP could be evoked by the intervention.

In the next phase, the data extracted from the literature were complemented by the clinical expertise of therapists and patient preferences

\section{Clinical Expertise of Therapists and Patient Preferences}

Eleven patients ( 6 female) and 10 therapists $(8$ female) were recruited for the interviews until saturation of data was achieved. The sample

of therapists consisted of 5 occupational and 5 physical therapists (age range 23 t0 57) with a range in work experience from 5 to 28 years of three therapists worked in a hospital 4 an a rehabilitation center and 3 ing a 23 rivate practice. The therapists used MT for 2 to 5 years and the majority hed treated at 30 patients with PLP during the past 12 moths and between 5 and 20 patients in total One therapist working an academic hospital had treated more than 100 patients.

The sample of patients was very heterogeneous as shown in Table 2. Only 3 patients were currently using MT either as a self-management or as guided individual treatment.
The results of the questionnaires and interviews showed that the majority of therapists used a similar approach of applying MT in patients with PLP. First, therapists screened eligibility of patients by applying several selection criteria such as sufficient cognitive abilties (eg, attention, working memory, and concentration) and the status of the intact limb or visual impairments. Eligible patients were then informed about the background of PLP and MT as well as possible side effects of the intervention.

\section{Table 2. Characteristics of Patients Participating in this Study}

\begin{tabular}{|c|c|c|c|c|c|c|c|c|c|}
\hline Patient & $\begin{array}{l}\text { Age } \\
\text { (in years) }\end{array}$ & 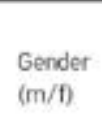 & $\begin{array}{l}\text { Work } \\
\text { Status }\end{array}$ & $\begin{array}{l}\text { Time Since } \\
\text { Amputation } \\
\text { (months) }\end{array}$ & $\begin{array}{l}\text { Side } \\
\text { Amputation }\end{array}$ & $\begin{array}{l}\text { Level } \\
\text { Amputation }\end{array}$ & $\begin{array}{l}\text { Reason } \\
\text { Amputation }\end{array}$ & $\begin{array}{l}\text { MT Sessions } \\
\text { Followed }\end{array}$ & $\begin{array}{l}\text { Stull Using } \\
\text { MT }\end{array}$ \\
\hline $\begin{array}{l}1 \\
2 \\
3 \\
4 \\
5 \\
6 \\
7 \\
7 \\
8 \\
9 \\
10 \\
11\end{array}$ & $\begin{array}{l}22 \\
49 \\
56 \\
56 \\
49 \\
70 \\
39 \\
49 \\
49 \\
59 \\
59 \\
24\end{array}$ & $\begin{array}{l}F \\
M \\
F \\
M \\
F \\
M \\
M \\
F \\
M \\
M \\
F \\
F\end{array}$ & 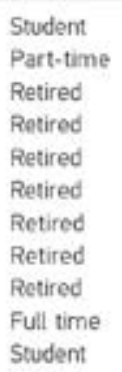 & $\begin{array}{r}15 \\
12 \\
5 \\
116 \\
27 \\
36 \\
39 \\
328 \\
35 \\
3 \\
45\end{array}$ & $\begin{array}{l}\text { Leth } \\
\text { Right } \\
\text { Right } \\
\text { Right } \\
\text { Right } \\
\text { Leth } \\
\text { Leth } \\
\text { Right } \\
\text { Right } \\
\text { Right } \\
\text { Left }\end{array}$ & 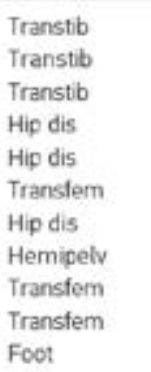 & 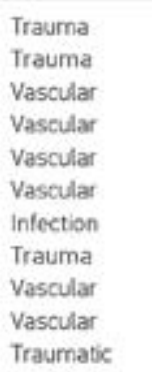 & $\begin{array}{r}6 \\
39 \\
8 \\
3 \\
6 \\
12 \\
8 \\
5 \\
5 \\
50 \\
3 \\
15\end{array}$ & \\
\hline
\end{tabular}

Before treatment started, all therapists assessed PLP (eg, intensity, localization, duration) and aspects of the phantom limb (eg, position, range of motion). Three therapists also systematically assessed limitations in daily activities and participation.

Additional aspects that were not mentioned in the literature concerned the use of trigger point therapy in case of perceived malposition of the phantom limb, as this could normalize the perceived position of the phan limb and thereby facilitate the effects of MT. In adtion of the positioning of the patient in front of the mirror, the majority of theraists asked patients to place the intact limb in the same position as the phantom limb in order to facilite the mirror illusion. 
After this initial "creation" of the mirror illusion, all therapists used exercises from 4 different categories: Basic motor exercises without using objects (eg, flexion/ extension movements), sensory stimuli (eg, brushes, vibration, warmth), functional motor exercises using objects (eg, grasping marbles with the toes) and mental practice of motor exercises (facilitated with or without the mirror). Most of the therapists used
voluntary movements of only the intact limb first, and in case patients were also able to perform pain-free movements with their phantom limb, voluntary movements of the phantom limb were initiated.

Only one therapist additionally used limb laterality recognition training as recommended in the study of Moseley as part of graded motor imagery program 20 All therapists agreed that a tailor-made exercise program depending on patient preferences should be used and that self-management of patients should be facilitated as soon as possible The majority of therapists used pain diaries and logs to monitor selfmanagement and evaluate the effects of the intervention.

\section{Patient Preferences}

Additional information was derived from the patients' interviews referring to the categories of the theoretical model "general requirements," quotes made by patients.

With regard to the category "general requirements," the majority of patients was skeptical about the treatment when MT was introduced of 2 intact limbs and the real situation of only one limb being present which evoked emotional reactions.

On the one hand you have to accept that you don't have a left foot anymore, but then

the

foot suggesting it is still present...

Patients suggested that sufficient information about the background and relation between amputation, PLP and MT, success stories of other patients, and a clear formulation of treatment aims should be used to facilitate patient engagement in MT.
With regard to the category "treatment," many patients had problems to create the "mirror illusion" in the beginning of the treatment, which means that they did not perceive the limb in the mirror as their affected limb (embodiment of the mirror image). One patient mentioned that the malposition of her phantom limb prevented her from creating the illusion, as the intact limb was not positioned in a similar way, which is in correspondence with the literature. $26,3,3,33$

I perceived my phantom leg behind the mirror as being strutted apart and this didn't

match with the mirror image. I rather had the feeling of having 3 legs.

As the vividness of the mirror illusion seems to be correlated with the effects of the training, ${ }^{21}$ it is important to facilitate embodiment of the mirror image. This could be achieved by adopting a similar position with the intact limb as perceived in the phantom limb. $26.3 .1,33$ Furthermore, 2 patients indicated that the mirror illusion was facilitated through the instruction of the therapist to imagine looking through a window instead of a mirror and by intense focusing of the mirror image as well as fading out the image of the intact limb. However, another patient mentioned that the starting position of the intact limb was of minor importance for him because the perception of the phantom limb immediately adapted to the mirror image.

...the moment I am looking into the mirror I don't feel the faulty position of the phantom

any more... the phantom adopts the posture simulated by the mirror image.

The majority of patients also mentioned that they achieved more effects through passive sensory stimuli than through self-administered sensory stimuli. It was also indicated by 2 patients that therapists should carefully apply these sensory stimuli because a too intense treatment dose could result in side effects such as increased cramping and pain.

...otherwise I had the feeling of stimulus satiation and then it (the phantom) clenched.

For that reason, the content and treatment dose of the exercise program should be adapted to the individual preferences of the patient. With regard to motor exercises 4 patients emphasized that movements should in the beginning be performed with the intact limb only, as 
voluntary movements of the phantom limb are more effortful and sometimes even evoked cramping and PLP. The latter was the case in 2 patients who were not able to voluntary move the phantom but were asked to do so.

I. was told to move the toes up and as soon as I had elevated the toes, I usually wasn't

able to move them down again and they began to cramp.

In addition to the literature, many patients reported additional positive effects of the intervention such as improved body image and self-efficacy or a decrease in medication intake. With regard to potential side effects of the intervention (eg, dizziness, nausea) 3 patients confirmed the findings from the literature. However, patients also mentioned that most of the side effects resolved through the first sessions.

This is like getting new glasses, you have to get accustomed to it.

One patient recommended the following graded approach in case of side effects such as nausea or dizziness: Patients should be instructed to observe the miror image only over a short period of time and then turn their gaze away toward the unaffected limb This procedure should be repeated several times, until the side effects resolve.

\section{Categories of the Clinical Framework}

For every main category of the a priori formulated theoretical model, several subcategories emerged from the literature search patient and therapist interviews as shown in Figure 2.

The number of subcategories per main category varied from 2 to 5 . The main category "general requirements," for example, was divided into the subcategories "patient characteristics" "environmenta information" "The main category "evaluation" was divided into the subcategories "freeuency and quality of exercises" and "(side) effects." Some subcategories were further divided into more subcategories. The main categor "effects" for example was divided into the subcategories "positive and negative effects of the intervention" Five addition subcategories were found for the categor "positive effects" and 3 additionat subcategories for the category "negative effects."
Clinical Flow Chart

Based on these categories, we developed a clinical flowchart that incorporates the main and subcategories in a logical way according to the phases in methodical intervention defined by the Royal Dutch Society for Physical Therapy 28.46 This clinical flowchart (see Figure 3) allows therapists to directly implement the intervention in their daily routine. We then used detailed information mainly from the interviews with therapists to complement each category to create the final clinical framework as a comprehensive booklet that illustrates the individual steps of the clinical flowchart (see Appendix).

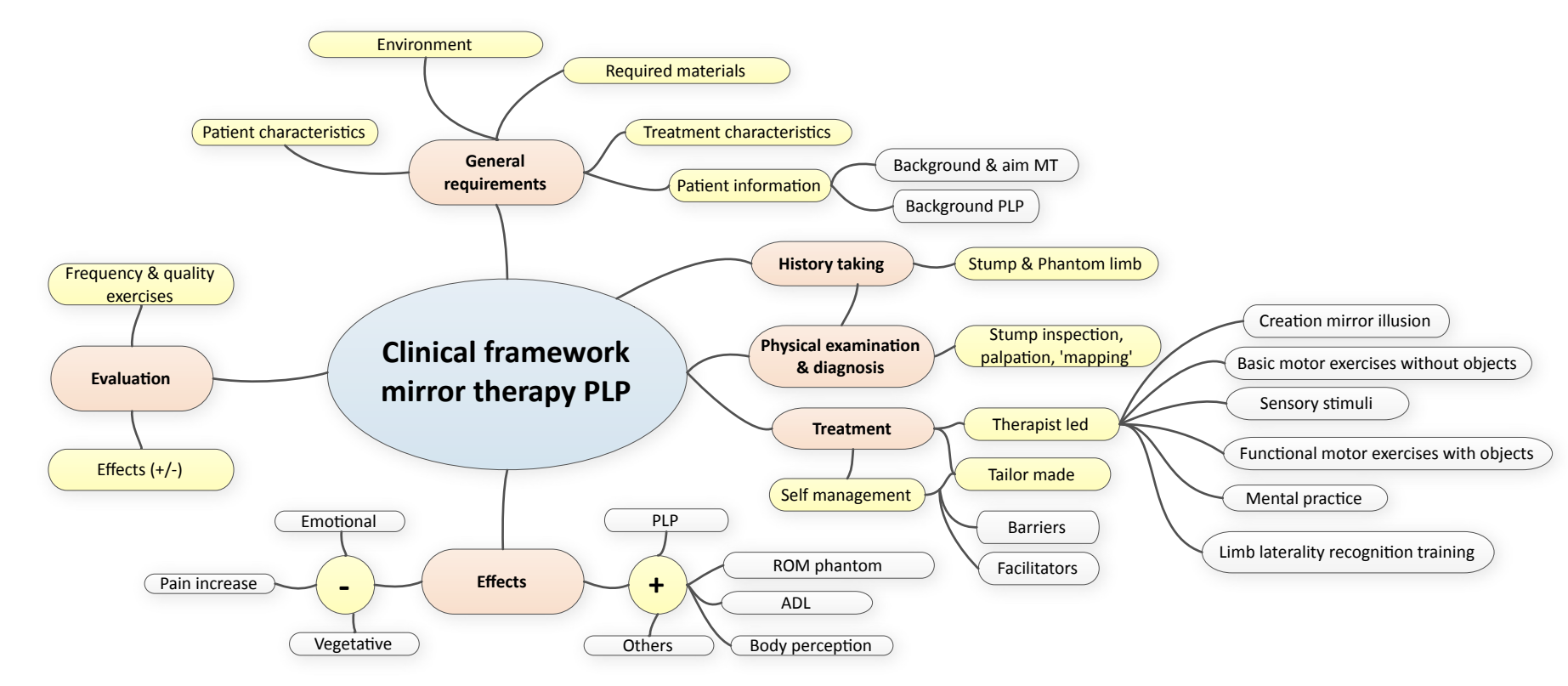

Figure 2. Mind map of main and subcategories of the clinical framework 


\section{DISCUSSION}

The aim of this article was to describe the development of a clinical framework for MT in patients with phantom limb pain following amputation. We tried to incorporate the best available evidence, clinical experiences of therapists, and preferences of patients suffering from PLP who have experience in using MT. The available literature shows moderate quality of evidence that MT is effective as an additional intervention in improving recovery of arm and hand function after stroke. 22.47.48 Despite its promising results in existing PLP clinical trials, the quality of evidence in patients with PLP is still low. ${ }^{22}$ Nevertheless, MT is increasingly being used in clinical practice, and its effects on PLP and cortical plan for MT in patients with PLP is still missing. Our clinical framework contributes to a more structured approach of applying MT in clinical practice and could also be used in upcoming framework is still weak, with only 2 controlled trials 1820 investigating effects of MT in patients with PLP.

\section{(In)congruence between Literature and Interview Data}

Remarkably, many of the data drawn from the literature matched with the information derived from interviews with therapists and patients. For example many therapists highlighted the importance of establishing and assessing the vividness of the mirror illusion, which is in line with results from the literature 21.26.3.141.142 Furthermore, the majority of therapists also used the different exercise categories described above that evolved through the analysis of the literature. As described above, patients reported that voluntary movements of the phantom limb could result in an increase in cramping and pain when the range of motion or complexity of the task far exceeds the motor ability of the phantom limb For the same reason, many therapists asked patients to voluntary move their phantom only within the individual painfree range of motion of the phantom limb. This finding is in line with the studies of Glaudo $0^{42}$ and Mercier et al ${ }^{31}$ who also point out that . increased feeling of cramping and pain. Similar observations have been made by Moseley et al ${ }^{50}$ who showed that motor imagery of specific

the literature, $39,0,0,2,4,4,51$ one should systematically assess such negative effects and sufficiently inform patients before starting the treatment.

Interestingly, only one therapist used limb laterality recognition training, which is recommended within the graded motor imagery approach. 20.45
In the protocols of Mich ${ }^{4 l}$ and Glaudo, ${ }^{42}$ limb laterality recognition training is seen as complementary tool for some patients. For this reason we included limb laterality recognition training as optional part in our framework.

The results also showed that therapists should take the individual preferences of patients for motor and sensory stimuli into account. Some patients did respond more to specific sensory stimuli (eg, brushes or warmth) and experienced bigger effects than to motor exercises, which is in line with the findings of Schmalzz et a ${ }^{2}{ }^{26}$ In our view, it is important to create an individual tailored exercise program consisting of exercises from different categories matching the individual preferences of the patient which corresponds with existing treatment protocols 39.22

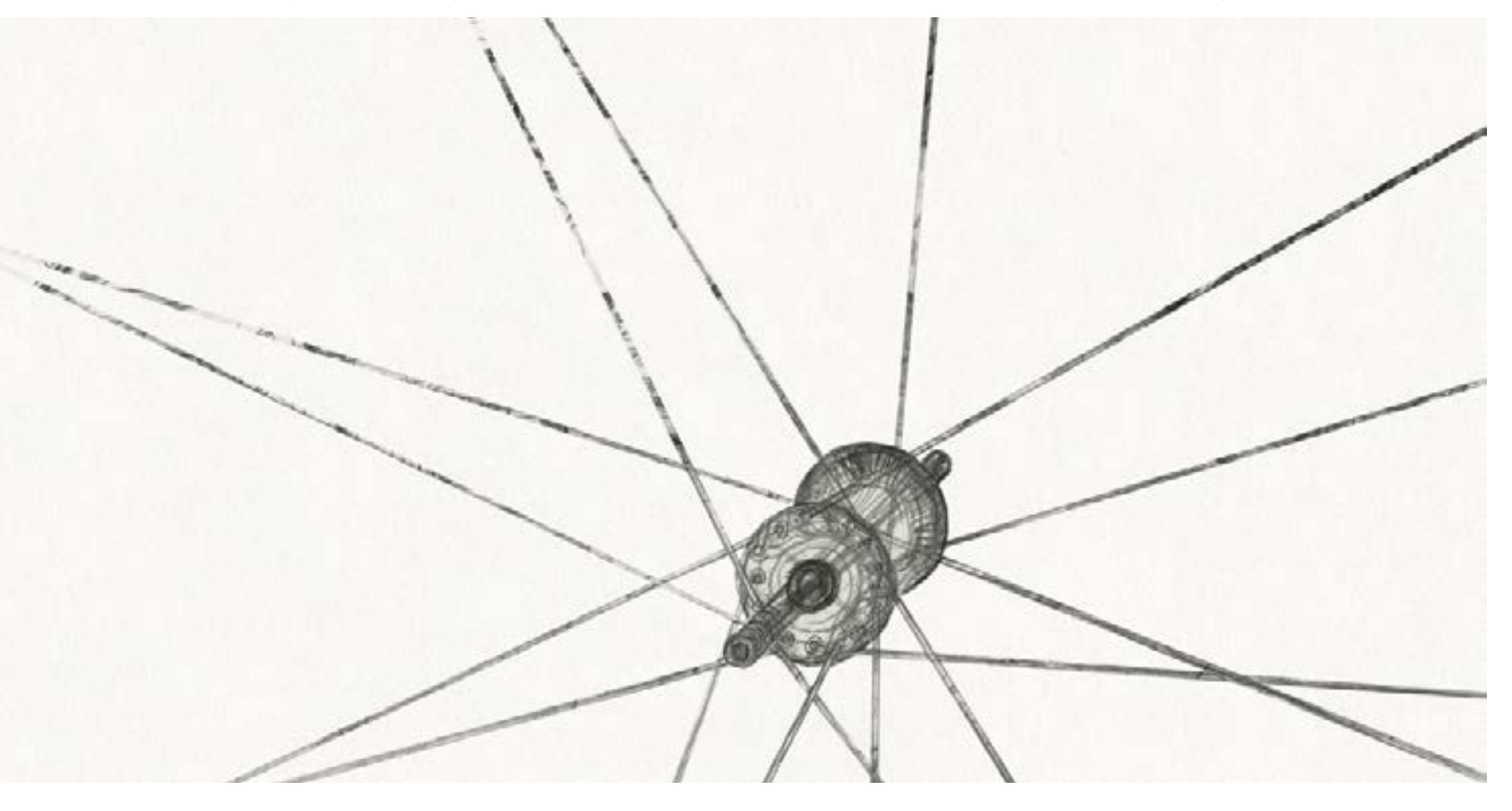




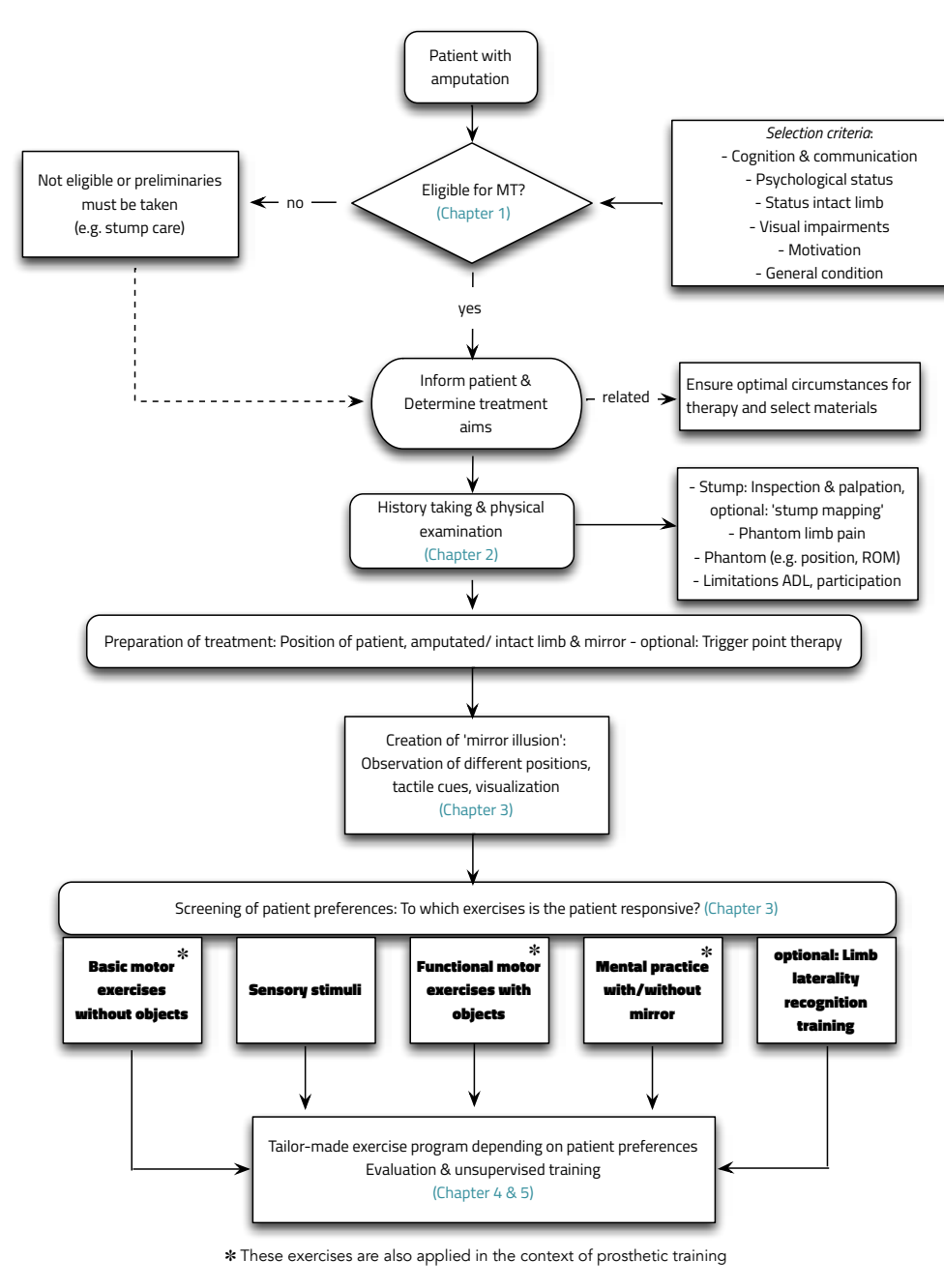

Figure 3. Flowchart of the clinical framework based on main and subcategories.
User-centered Approach

Data regarding our theoretical model that could be extracted from clinical trials were sparse. Therefore, additional clinically important information was collected through a user-centered approach using questionnaires and interviews with patients and therapists to complement the information extracted from the literature.27 However, therapists were mainly recruited via existing networks of one of the authors (AR), introducing the risk of selection bias. The inclusion of a more diverse international group of experts such as in the study of Hagenberg et al.4.4 could have led to other results.

Detailed vs. More General Framework

In the beginning of our study, we deliberated about whether we should develop a more detaled or general clinical frameworksimilartothestudies more teares more freedom for persona completion. In adion. leaves more freedom for personal completion. In addition, we realized that the practical protocol we developed for MT in stroke patient $\mathrm{s}^{53} \mathrm{met}$ the needs of many therapists as it is frequently downloaded and bookmarked. This gave reason to use a similar approach for the development of a clinical framework for MT in patients with PLP. However, a too detailed framework bears the risk of guiding therapists too much into one specific direction and to limit the incorporation of their own clinical experiences. Therefore, on the one hand, we tried to guide therapists through the whole clinical process from patient intake to discharge but on the other hand leave enough space for individual adaption.

Final Remarks

We are currently testing our clinical framework for its effectiveness in patients with PLP following lower limb amputation in a multicenter randomized controlled trial. . $^{9}$ In preparation of this trial, it was important to develop a structured protocol in order to instruct therapists how to deliver the intervention in a standardized way.

In the same trial, we are evaluating the additional effects of a telerehabilitation including, among other treatments, a novel "mobile" approach to MT. In this method, the tablet-integrated camera detects movements of the intact limb and displays them as movements of the amputated limb (Figure 4). Preliminary results from our trial suggest that the effects of this new approach on PLP are comparable to the effects of traditional MT. Finally, through our multicenter trial, we hope to gain more insight into the practicability and clinical relevance of our clinical framework.

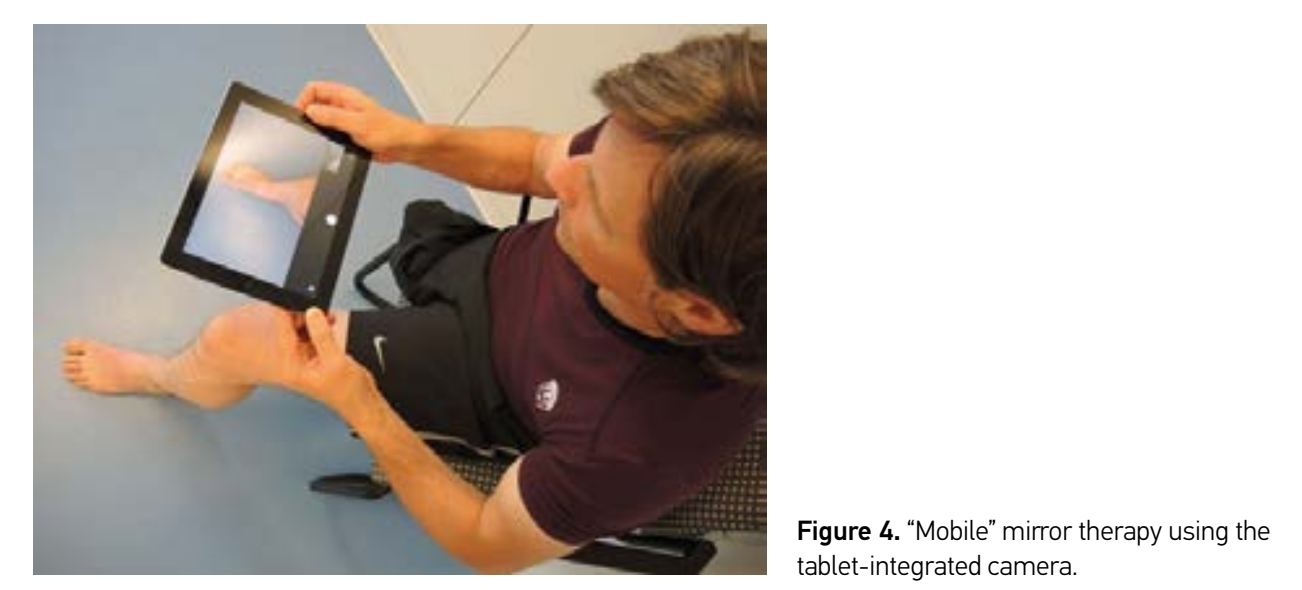




\section{REFERENCES}

Ephraim PL, Wegener ST, Mackenzie EJ, Dillingham TR, Pezzin LE. Phantom pain, residual limb pain, and back pain in amputees: results of a national survey. Arch Phys Med Rehabil. 2005;86:1910-1919. Kern U, Busch V, Rockland M, Kohl M, Birklein F. Prevalence and risk factors of phantom limb pain and phantom limb sensations in Germany. A nationwide field survey. Schmerz. 2009;23:479-488. Kooijman CM, Dijkstra PU, Geertzen JH, Elzinga A, Van Der Schans CP. Phantom pain and phantom sensations in upper limb amputees: an epidemiological study. Pain. 2000;87:33-41. Richardson C, Glenn S, Nurmikko T, Horgan M. Incidence of phantom phenomena including phantom limb pain 6 months after major lower limb amputation in patients with peripheral vascular disease. Clin J Pain. 2006;22:353-358.

Bosmans JC, Suurmeijer TP, Hulsink M, Van Der Schans CP, Geertzen JH, Dijkstra PU. Amputation, phantom pain and subjective well-being: a qualitative study. Int J Rehabil Res. 2007;30:1-8. Hanley MA, Ehde DM, Jensen M, Czerniecki J, Smith DG, Robinson LR. Chronic pain associated with upper-limb loss. Am J Phys Med Rehabil. 2009;88:742-751.

Sinha R, van den Heuvel WJ, Arokiasamy P. Factors affecting quality of life in lower limb amputees. Prosthet Orthot Int. 2011;35:90-96.

Van der Schans CP, Geertzen JH, Schoppen T, Dijkstra PU. Phantom pain and health-related quality of life in lower limb amputees. J Pain Symptom Manage. 2002;24:429-436.

Griffin SC, Tsao JW. A mechanism-based classification of phantom limb pain. Pain. 2014;155:2236-2242 Abbass K. Efficacy of gabapentin for treatment of adults with phantom limb pain. Ann Pharmacother. 2012;46:1707-1711.

Barrett AM, Lucero MA, Le T, Robinson RL, Dworkin RH, Chappell AS. Epidemiology, public health burden, and treatment of diabetic peripheral neuropathic pain: a review. Pain Med. 2007;8(suppl 2):S50-S62. Alviar MJ, Hale T, Dungca M. Pharmacologic interventions for treating phantom limb pain. Cochrane Database Syst Rev. 2011;(12):CD006380.
3. Birbaumer N, Lutzenberger W, Montoya P, et al. Effects of regional anesthesia on phantom limb pain are mirrored in changes in cortical reorganization. J Neurosci. 1997;17:5503-5508.

14. Flor $\mathrm{H}$, Elbert $\mathrm{T}$, Knecht S, et al. Phantom-limb pain as a perceptual correlate of cortical reorganization following arm amputation. Nature. 1995:375:482-484.

15. Karl A, Birbaumer N, Lutzenberger W, Cohen LG, Flor H. Reorganization of motor and somatosensory cortex in upper extremity amputees with phantom limb pain. J Neurosci. 2001;21:3609-3618.

Koppelstaetter F, Siedentopf CM, Rhomberg P, et al. Functional magnetic resonance imaging before motor cortex stimulation for phantom limb pain. Nervenarzt. 2007;78:1435-1439.

17. Flor H, Nikolajsen L, Staehelin Jensen T. Phantom limb pain: a case of maladaptive CNS plasticity? Nat Rev Neurosci. 2006; 7:873-881.

18. Chan BL, Witt R, Charrow AP, et al. Mirror therapy for phantom limb pain. N Engl J Med. 2007;357:2206- 2207. Maclver K, Lloyd DM, Kelly S, Roberts N, Nurmikko T. Phantom limb pain, cortical reorganization and the therapeutic effect of mental imagery. Brain. 2008;131(Pt 8):2181- 2191.

20. Moseley GL. Graded motor imagery for pathologic pain: a randomized controlled trial. Neurology. 2006;67:2129-2134.

21. Foell J, Bekrater-Bodmann R, Diers M, Flor H. Mirror therapy for phantom limb pain: brain changes and the role of body representation. Eur J Pain. 2014;18:729-739.

22. Rothgangel AS, Braun SM, Beurskens AJ, Seitz RJ, Wade DT. The clinical aspects of mirror therapy in rehabilitation: a systematic review of the literature. Int J Rehabil Res. 2011;34:1-13.

23. Darnall BD. Self-delivered home-based mirror therapy for lower limb phantom pain. Am JPhys Med Rehabil. 2009;88:78-81.

24. Maclachlan M, McDonald D, Waloch J. Mirror treatment of lower limb phantom pain: a case study. Disabil Rehabil. 2004;26:901-904.

25. Darnall $\mathrm{BD}, \mathrm{Li}$ H. Home-based self-delivered mirror therapy for phantom pain: a pilot study. J Rehabil Med. 2012;44:254-260.

26. Schmalz L, Ragno C, Ehrsson HH. An alternative to traditional mirror therapy: illusory touch can reduce phantom pain when illusory movement does not. Clin J Pain. 2013;29: e10-e18. 
27. Sackett DL, Rosenberg WM, Gray JA, Haynes RB, Richardson WS. Evidence based medicine: what it is and what it isn't. BMJ. 1996;312:71-72.

28. de Vries C, Hagenaars L, Kiers H, Schmitt M. The Professional Profile of the Physical Therapist. Amersfoort Royal Dutch Society for Physical Therapy; 2006 .

Hsieh HF, Shannon SE. Three approaches to qualitative content analysis. Qual Health Res. 2005:15:1277-1288. Brodie EE, Whyte A, Niven CA. Analgesia through the looking-glass? A randomized controlled tria J Pain. 2007;11:428-436.

31. Mercier C, Sirigu A. Training with virtual visual feedback to alleviate phantom limb pain. Neurorehabll Neural Repair. 2009;23:587-594

32. Giraux P, Sirigu A. Illusory movements of the paralyzed limb restore motor cortex activity. Neurolmage 2003;20 (suppl 1):S107-S111.

33. Seidel S, Kasprian G, Furtner J, et al. Mirror therapy in lower limb amputees - a look beyond primary motor cortex reorganization. Rofo. 2011:183:1051-1057.

34. Hanling SR, Wallace SC, Hollenbeck KJ, Belnap BD Tulis MR. Preamputation mirror therapy may prevent development of phantom limb pain: a case series. Anest Analg. 2010;110:611-614.

35. Brodie EE, Whyte A, Waller B. Increased motor control of a phantom leg in humans results from the visual feedback of a virtual leg. Neurosci Lett. 2003;341:167-169.

Sumitani M. Miyauchi S. MCCabe CS, et al. Mirror visual feedback alleviates deafferentation pain, depending on qualitative aspects of the pain: a preliminary report. Rheumatology (Oxford). 2008;47:1038-1043. Kim SY, Kim YY. Mirror therapy for phantom limb pain. Korean J Pain. 2012;25:272-274

38. Wilcher DG, Chernev I, Yan K. Combined mirror visual and auditory feedback therapy for upper limb phantom pain: a case report. J Med Case Rep. 2011;5:41.

Gruenert-Pluess N. Hufschmid U. Santschi L., Gruenert 」 Mirror therapy in hand rehabilitation: a review of the literature, the St Gallen protocol for mimor therapy and evalualonc Br J Hand Ther. 2008; 13:4-9.
McCabe CS. Mirror visual feedback therapy. A practical approach. J Hand Ther. 2011;24:170-178. Michl K, Kraft E. Mirror therapy in the treatment of phantom limb pain. ZKM. 2014;5:48-52. Glaudo S. Mirror therapy in pain patients. Praxis Ergotherapie. 2010;23:268-273.

Schwarzer A, Glaudo S. Zenz M, Maier C. Mirror feedback - a new method for the treatment of neuropathic pain. Dtsch Med Wochenschr. 2007;132:2159-2162.

44. Hagenberg A, Carpenter C. Mirror visual feedback for phantom pain: international experience on modalities and adverse effects discussed by an expert panel: a Delphi study. PM R. 2014;6:708-715. randomised controlled trial. Pain. 2004;108:192-198.

46. Heerkens YHH de Graaf-Peters V. KNGF-Richtlin Fysiotherapeutische Verslaglegging. Amersfoort Koninklijk Nederlands Genootschap voor Fysiotherapie; 2011.

47. Thieme H, Mehrholz J, Pohl M, Behrens J, Dohle C. Mirror therapy for improving motor function after stroke. Cochrane Database Syst Rev. 2012: (3):CD008449.

4. Veerbeek JM, van Wegen $\mathrm{E}$, van Peppen $\mathrm{R}$, et al. What is the evidence for physical therapy poststroke? A systematic review and meta-analysis. PLoS ONE. 2014;9: e87987.

49. Rothgangel AS, Braun S, Schulz RJ, et al. The PACT trial: PAtient Centered Telerehabilitation: Effectiveness of software-supported and traditional mirror therapy in patients with phantom limb pain following lower limb amputation: protocol of a multicentre randomised controlled trial. J Physiother. 2015:61:42.

50. Moseley GL, Zalucki N Birklein F Marinus J, van Hilten Jt, Luomajoki H. Thinking about movement 2008:59: 623-631.

51. Casale R. Damiani C, Rosati V. Mirror therapy in the rehabilitation of lower-limb amputation: are there any contraindications? Am J Phys Med Rehabil. 2009;88:837-842.

52. Braun S, Kleynen M, Schols J, Schack T, Beurskens A, Wade D. Using mental practice in stroke rehabilitation. a framework Clin Rehabil 2008:22579-591. D

53. Rothgangel AS, Braun SM. Mirror Therapy: Practical Protocol for Stroke Rehabilitation. Munich: Pflaum Verlag; 2013 


\section{APPENDIX CHAPTER 3}

MIRROR THERAPY

Clinical framework for treatment of phantom limb pain

Andreas Rothgangel, Susy Braun, Luc de Witte

Anna Beurskens, Rob Smeets

Pain Practice, 2016;16(4):Suppl 1 
Preface

This clinical framework for the application of mirror therapy in patients with phantom limb pain after amputation was developed in preparation of the PACT (PAtient Centered Telerehabilitation) trial. In this controlled clinical trial the effectiveness of mirror therapy supported by telerehabilitation with regard to the intensity, duration and frequency of phantom limb pain and daily activities is assessed in patients following lower limb amputation. This experimental intervention is compared to both traditional mirror therapy and usual care without mirror therapy. Many variations in applying mirror therapy exist whereas detailed information and a standardized, evidence based treatment protocol for mirror therapy in patients with phantom limb pain is missing. Therefore, a structured protocol was developed in order to instruct therapists how to delver mirror therapy in a standardized way in a prelminany phase. This evidence based clnical framework was not only developed to serve as a structured guideline for therapists who deliver the treatment but also to support implementation of mirror therapy in routine care.

Three sources of data collection (in accordance with the evidence based practice approach²) were used to develop this clinical framework: We reviewed the literature on important clinical aspects regarding mirror therapy and the evidence on the effectiveness in patients with phantom limb pain. In addition, we used questionnaires and semi-structured interviews in both patients with phantom limb pain who already had experience with mirror therapy and physical and occupational therapists to assess their experiences and preferences regarding the application of mirror therapy.

Comparable to almost all specific rehabilitation interventions, effect sizes for mirror therapy are still relatively small and new evidence might overturn existing evidence. Mirror therapy should therefore be considered as one of several therapy interventions within a rehabilitation programme to reduce phantom limb pain in which other interventions can be offered as well, or sometimes may even be preferred.

The present protocol should be seen as a framework, not a predefined recipe for all patients. Within the protocol the basic principles and many examples of how to apply mirror therapy are given. The framework however leaves enough room for the therapist to adjust the mirror therapy treatment for each individual patient.
We hope that this clinical framework facilitates the tailored treatment of patients suffering from phantom limb pain with mirror therapy in routine care.

\section{Acknowledgemen}

We would like to thank all patients and therapists who were involved in the development of this framework. Thank you for sharing you valuable experiences and thoughts with us.

Andreas Rothgangel, Susy Braun, Luc de Witte, Anna Beurskens and Rob Smeets January 2015 


\section{CONTENT}

INTRODUCTION

CHAPTER 1 General requirements

CHAPTER 2 History taking $\&$ physical examination

CHAPTER 3 First therapy sessions

CHAPTER 4 Developing a tailored exercise program

CHAPTER 5 Facilitating unsupervised training

\section{APPENDIX}

APPENDIX 1 Patient information sheet for mirror therapy

120

APPENDIX 2 Mirror therapy log

APPENDIX 3 Clinical flow chart mirror therapy 
One of the most important complaints of patients following amputation is the existence of phantom limb pain, which is perceived in the missing limb. Up to $80 \%$ of patients after amputation suffer from chronic phantom limb pain, ${ }^{3-6}$ leading to limitations in daily activities and quality of life.4.710 Despite the high number of phantom limb pain there is currently no standard effective treatment." Treatment of phantom limb pain mainly consists of pain medication despite potential side effects, ${ }^{12}$ high $\operatorname{costs}^{13}$ and only low quality of evidence regarding its longterm efficacy. ${ }^{14}$

Reorganization of the somatosensory $y^{15,16}$ and motor cortex $x^{17,18}$ has been proposed to contribute to phantom limb pain. It was shown that the invasion of cortical areas neighbouring the representation of the amputated limb positively correlates with the intensity of phantom limb pain. ${ }^{19}$ In this context alternative, non-pharmacological interventions such as mirror therapy are gaining increased attention in the treatment of phantom limb pain.20-22 During mirror therapy the patient sits in front of a mirror that is oriented parallel to the patients' midline and consequently blocks the view of the amputated limb (fig. 1). This arrangement facilitates an illusion of two existing intact limbs that can therapeutically be used to reverse cortical reorganization and thereby reduce phantom limb pain. ${ }^{27}$ In a recent systematic review ${ }^{24}$ it has
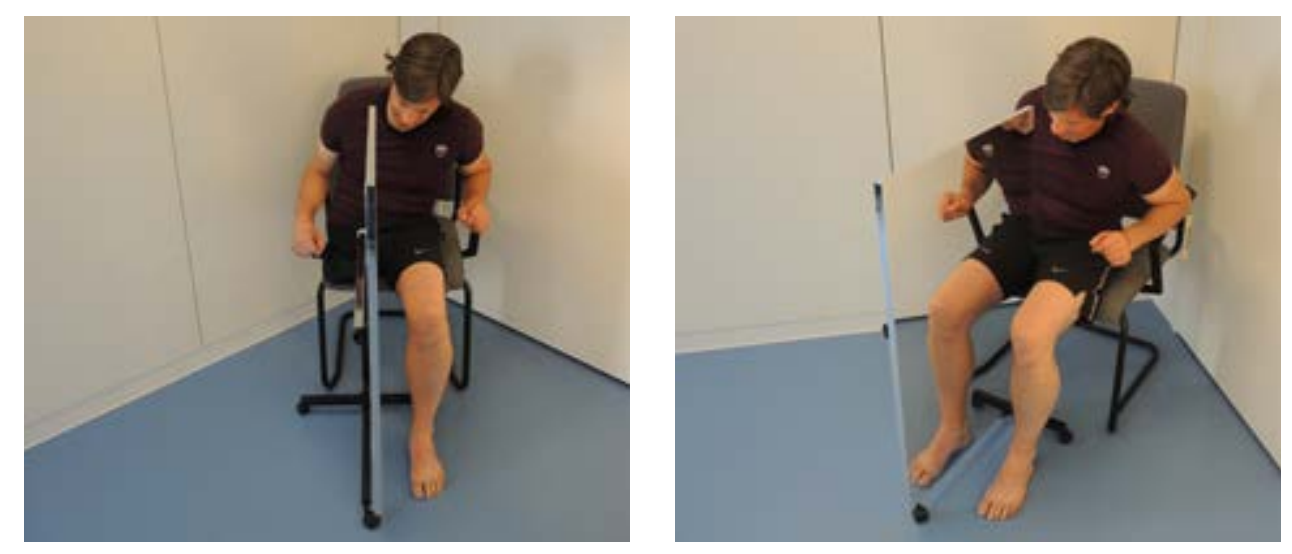

been reported that despite the potential merits of mirror therapy the quality of evidence in patients with phantom limb pain is still low and detalled description of how to deliver mirror therapy is missing. In addition, interventions do not seem to be comparable, because data on mportant clinical aspects of mirror therapy, such as patien

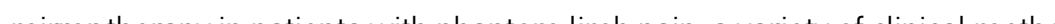
of mirror therapy and mental pact exercises using sensory stimulation seem to be equally important.2. whereas detaledinformation and a sandandzed, evidence basedr. missing. Therefore, we developed this evidence based clinical framework to support structured and standardized implementation of mirror therapy in clinical care.

This protocol was specifically designed according to the different steps of methodical intervention of therapists defined by the Royal Dutch Society for Physical Therapy $y^{29}$ to facilitate embedment of mirror therapy into daily practice. These steps include information on selecting and informing eligible patients, history taking and physical examination, diagnosis and indication for treatment, treatment (plan) and evaluation of the treatment. These steps are also in line with the process of clinical reasoning and we hope that this will facilitate quick and easy orientation. allowing therapists to get a generalidea about the basic approach when using mirror therapy in patients with phantom limb pain following amputation.

Notes: The emphasis of this clinical framework is on the lower limb as the majority of patients suffer from amputations of the lower limb. However, the principles described in this protocol also apply to the upper limb The examples are given to show the scope of application possibilities.

Figure 1. The principle of mirror therapy 

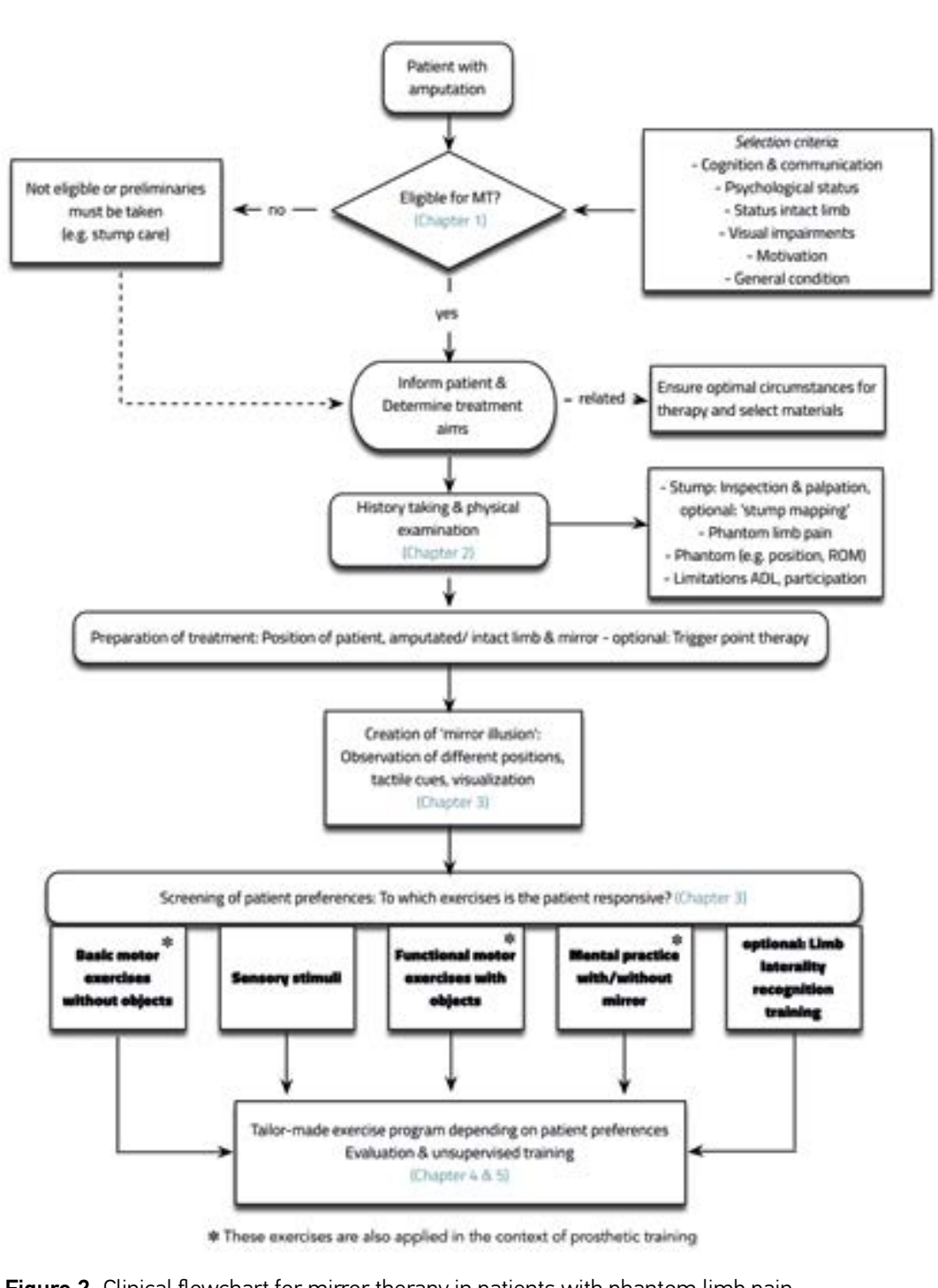

Chapter 1: General requirements

First, characteristics that are important when choosing eligible patients are described, followed by aims of the

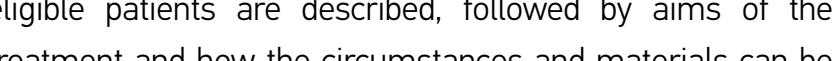
cosen in retrion to the cicums chesen diferent interventioncharactict before starting treatment. Figure 2 gives an overview of the entire clinical process from patient selection to the design of a tailored exercise program. An addition in the form of a removable version of this clinical flow-chart is given in the appendix of this framework.

\section{Patient characteristics}

The following patient characteristics are important to consider when choosing patients for a mirror therapy treatment These characteristics were derived for publisted sudies and clinical exp he sefria

Cognitive \& communicative abilities

Eligible patients should have sufficient cognitive and communicative abilities (e.g. attention, working memory and concentration) to focus at least for ten minutes on the mirror reflection and follow instructions given by the therapist. The treating therapist should make a clinical judgement, whether the patient has sufficient understanding of the background and aim of the intervention. It is favorable if patients are able to engage in this kind of treatment and to imagine the mirror image as their affected side as the vividness of the mirror illusion (defined as the feeling that the mirror image is part of one's body), seems to be correlated with the effects of the training. ${ }^{23}$

Psychological status

Patients with mental disorders (e.g. post-traumatic stress disorders) should only perform mirror therapy after prior assessment through psychologist, as the mirror image of two intact limbs might elicit memories associated with the trauma and thereby could evoke emotional

Condition of intact limb

The intact limb should ideally have a normal and pain-free range of motion. Severe constraints of the intact limb (e.g. range of motion, pain) could hamper execution of mirror therapy exercises. The same applies to severe alterations in visual image of the intact limb such as extensive scars following burns. The mirror image should match the perception of the affected limb as much as possible in order to facilitate the vividness of the mirror illusion. This means that all visual marks such as jewellery, tattoos or scars should be removed or covered before starting the treatment as far as it hinders the patient when looking into the mirror.

Vision

In case of visual impairments, therapists should determine if a patient can see a clear image of the entire limb and its movements in the mirror.

General condition

The patients' general condition should enable him to sit stable for the entire session, which could be restricted in the acute phase after amputation. Furthermore, very impatient and / or unsettled persons can experience difficulties with this kind of treatment, as it requires slow and focused execution of movements. 
Preliminary steps

In some patients mirror therapy might not be indicated at the moment due to limitations in (pain-free) sitting balance, coping with the disease or insufficient wound healing. In this case, additional preliminary steps should be taken such as residual limb care. Besides psychological interventions, residual limb care (e.g. applying cream and other sensory stimuli to the residual limb) and incorporation of the amputated limb in everyday activities as much as possible can be helpful to facilitate acceptance of the amputated limb.

\section{Aims of treatment}

In most cases the primary aim of the treatment is to decrease intensity and / or frequency and duration of phantom limb pain. A reduction in phantom limb pain often leads to oher desirable effects, such as a reduction in limitations of daily activities and participation (e.g. sleep, visiting friends). Based on the published literature and clinical experience, mirror therapy could also positively affect the following domains:

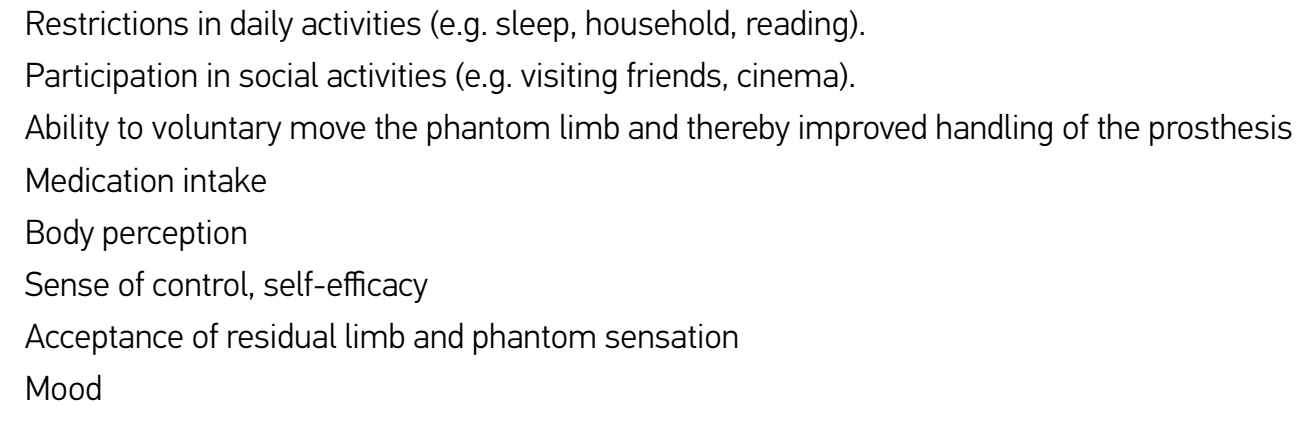

The individual aims have little impact on the structure and content of the exercises, with the exception of prosthesis training. In prosthesis training only motor exercises are used to improve motor control of the phantom limb.
Possible side effects

The mirror image of two intact limbs can evoke emotional reactions $30-32$ Other reactions like dizziness, nausea or sweating can be triggered in individual patients when observing the mirror reflection. In such cases, patients are instructed to no longer look into the mirror but to ing should then be instructed to observe the mirror image on then turn their gaze away towards the unaffected limb. This procedure should be repeated several times, until the side effects resolve. In case of persisting negative side effects it is recommended to stop the mirror therapy treatmen

\section{Informing the patient}

Before the first session, patients should be sufficiently instructed about the background and aims of mirror therapy as well as possible side effects of the treatment. In this context the mechanism of cortical reorganization ${ }^{16,17.33}$ in relation to the amputation and phantom limb pain can be explained using an illustration of the homunculus. The extent and detail of the information given depends on the cognitive abilities of the individual patient. Before the patient is seated in front of the mirror the principle of mirror therapy can first be demonstrated by the therapist himself. In addition, patients can be instructed to describe their perception of the intact and amputated limb with eyes closed to become aware of the discordance between how the limb is perceived by the brain and how it actually is ${ }^{34}$ The therapist could explain that

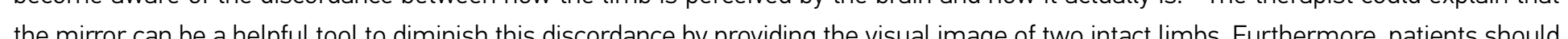
the mercen be a

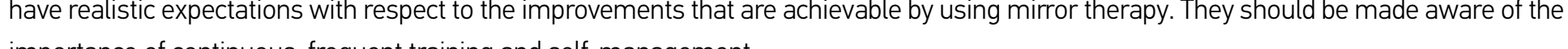
importance of continuous, frequent training and self-management. 
Environment and required materials

In this paragraph information with regard to the environment en required materials when applying mirror therapy is given.

\section{Surroundings}

As stated before, patients need to have sufficient attention and concentration when using mirror therapy, which implies that at least during the first sessions the environment should be free of other stimuli that might attract the patients' attention. For the same reason, at least the first sessions should be delivered individually instead of in a group, especially in easily distracted patients.

Jewellery and other marks

As described above there are indications that the mirror image should match the perception of the affected limb as much as possible in order to facilitate a vivid mirror illusion. ${ }^{23}$ This means that jewellery should be removed from both limbs before starting the treatment as far as it hinders the patient when looking into the mirror. The same applies to other visual marks on the intact limb such as birth marks, scars or tattoos that should be covered if they prevent a vivid image (e.g. with a plaster, glove or make-up).

Mirror

There are several mirrors commercially available, which are made of different materials (glass, foil, acrylic glass). When choosing a mirror one should pay attention to the following aspects:

- It should provide a coherent mirror image without any noteworthy distortion.

- There should be no risk of injury, e.g. through the edges of the mirror.

The mirror should be big enough to cover the entire affected limb and should allow patients to see all major movements in the mirror (fig. 3). A size of $25 \times 20$ inches $(60 \times 50 \mathrm{~cm})$ for the upper limb and at least $35 \times 25$ inches $(90 \times 60 \mathrm{~cm})$ for the lower limb should be large enough for everyday usage.

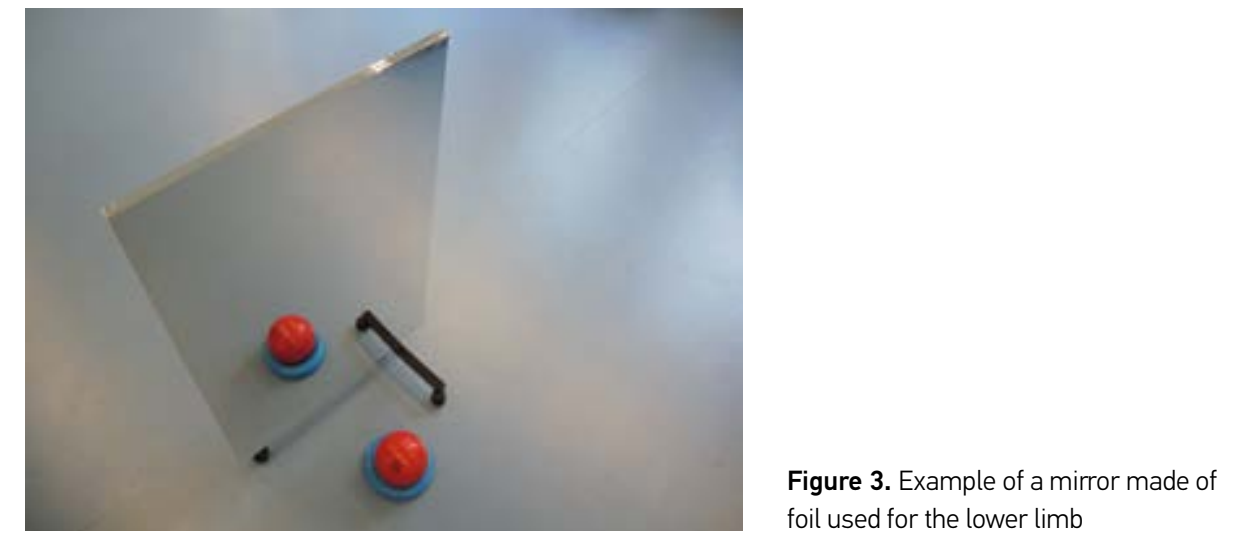

Exercise materials

For every patient, a tailored exercise program will be composed consisting of various motor and sensory exercises based on individual preferences. For this reason, various materials with more sensory input (fig. 4) should be used besides objects that are needed for functional motor training (e.g. cups, towels, marbles)

- Plastic bowl or tub filled with sand, rapeseed or peas

- Hedgehog ball

- Temperature stimuli (heat, cold)

- Different brushes

- Washing up gloves

- Vibration

- Wooden boards covered with different textures (e.g. fleece, sand paper, carpet)

- Cotton wool 
In addition, bedding materials such as cubes, sand- or balance pads can be used to position the lower limb, so that patients can see the entire limb more easily in the mirror and at the same time additional sensory stimuli are given.

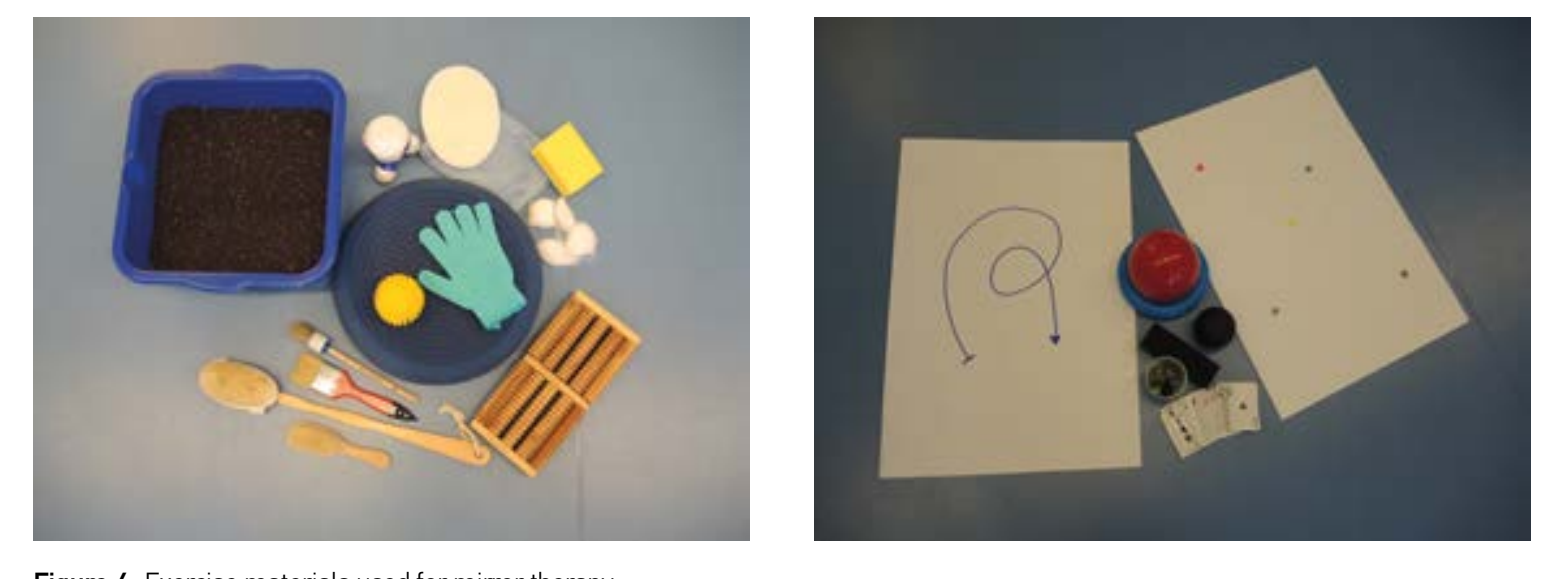

Figure 4. Exercise materials used for mirror therapy

\section{Treatment characteristics}

The paragraph on treatment characteristics is divided into aspects of the intensity of therapy and positions of the limbs and mirror.

Frequency of therapy and duration of sessions

The available literature ${ }^{24}$ recommends performing mirror therapy at least once a day with a minimum duration of ten minutes. The maximum Dossive side effects, but in most cases will range from 20 to 45 minutes. A daily treatment session using mirror therapy will be beyond the possibilities in many clinical settings. In such cases, patients will require instruction about unsupervised training using the mirror as early as possible within the treatment plan to enhance treatment intensity. Also, patients need a short instruction on how to use a corresponding log to monitor the intervention (see appendix 2 and chapter 5).

\section{Position of affected limb}

The patient sits in front of the mirror without wearing the prosthesis while the affected limb is situated in a safe and comfortable position behind the mirror. Occasionally, some patients are wearing their prosthesis during therapy in order to use the additional sensory input (e.g. approximation) for the exercises. For the same reason the lower limb is positioned in a closed-chain position in the beginning so that the foot has contact to the ground or balance pad respectively. In case of upper limb amputation, the affected limb should be positioned on a height adjustable table so that its position can be adjusted to the length of the patient's trunk and arm.

\section{Position of intact limb}

Some patients, in particular following traumatic injury, perceive their phantom limb in a malposition such as cramping or clenching. In such cases the natural position of the phantom limb can be adopted with the intact limb to facilitate the mirror illusion and can subsequently be used as starting point for the exercises. If patients do not indicate such a malposition of the phantom limb, the intact limb should be positioned in a way that matches the perception of the phantom as much as possible.

\section{Position of the mirror}

Generally, the mirror is positioned in front of the patient's midline, so that the affected limb is fully covered by the mirror and the reflection of the unaffected limb is completely visible (fig. 1). However, in some patients with malposition of the phantom limb it is important to ensure that the perceived position of the phantom limb can be adopted with the intact limb. In such cases the position of the mirror can be adjusted in such a way that it points more diagonally towards the intact limb. 
General therapy suggestions

Mainly based on cinical experience the following suggestions have been proven useful in order to achieve effective exercise performance and to avoid negative side effects such as an increase in pain:

- Start with simple motor and sensory exercises and slowly increase the complexity of

exercises towards more complex, functional exercises including objects.

- Try to incorporate tasks that are familiar to the patient within the exercise program

- When using bilateral movements, adjust the range and complexity of the movements

to the capacity of the phantom limb.

- Try to aim for an as high as possible number of repetitions (at least 15 reps per exercise), at the same time include variations of separate exercises with regard to range of motion, direction and starting position.

- In patients with better cognitive abilities unsupervised training is usually facilitated

- Pay close attention to a slow movement performance ("slow motion").

- Pay close attention to a slow movement performance ("slow motion").
Check the gaze direction of the patient regularly in the mirror and give feedback about the

exercise performance.

- Tailor the exercises to the patient's individual performance level.

- Try to create a tailored exercise program based on individual patient preferences.

Try to create a tailored exercise program based on individual patient preferences.
- Exercises should always be performed below the pain threshold; the intensity of phantom

- Exercises should always be performed below the pain threshold; the intensity
limb pain should also not be increased in the course after the treatment.

- Be careful to prepare the patient for the, real situation' at the end of a session (see 'ending

therapy sessions').

The length of a single session depends on the abilities of the patient. If necessary, incorporate sufficient breaks.
Ending therapy sessions

At the end of a therapy session patients should be prepared for viewing their amputated limb again when the mirror is removed. One possibility is to ask patients to direct their gaze away from the mirror image to the intact limb or another point in the room while preparing the patient verbally for the real image of the affected limb. Another possibility is to end the session with motor imagery exercise (see chapter 3) of the phantom limb with eyes closed. The entire treatment should be evaluated with appropriate measurement instruments (e.g. intensity of phantom limb pain and vividness of mirror illusion with NRS/VAS) 
After informing the patient about the background and aims of the treatment, history taking and physical examination takes place. In addition to specific questions regarding stump and phantom pain the therapist assesses the medical history as well as physical capacity and cognitive abilities of the patient With regard to the assessment of stump and phantom sensations the phantom and stump phenomena interview can be used.

History of phantom limb pain

History taking with respect to phantom limb pain includes questions regarding the localization, intensity and type of phantom and stump pain. In addition, the frequency and duration of pain episodes should be recorded, as well as the course of the phantom limb pain during the last week. Furthermore, provoking and relieving factors for phantom limb pain and limitations in daily activities and participation in social life should be assessed.

Phantom sensations

With regard to sensations in the phantom limb the following aspects should be assessed

- Posture, size and length of phantom limb (verbal description/demonstration through intact limb)

- Perceived pain-free active range of motion of phantom limb (verbal description/demonstration through intact limb)

- Localization and intensity of other, non-painful phantom sensations (e.g. cold, heat, tingling)

Inspection and palpation of amputated limb

Inspection of the stump includes assessment of wound healing around the localization of the scar and the condition of the skin in areas of high weight loading. Palpation can be used to localize trigger points or increased tone in the muscles of the stump area that could be related to the provocation of phantom limb pain.
Stump mapping

In some patients, specific stimulation points can be found in the stump that elicit referred sensations in the phantom limb. These points can be identified by 'brushing' or stroking the distal part of the stump using a small paintbrush. ${ }^{28}$ These points are marked on the stump and the corresponding parts of the intact limb (fig. 5) and are subsequently used for sensory stimulation exercises during the mirror therapy the corresponding parts
treatment (see chapter 3 .

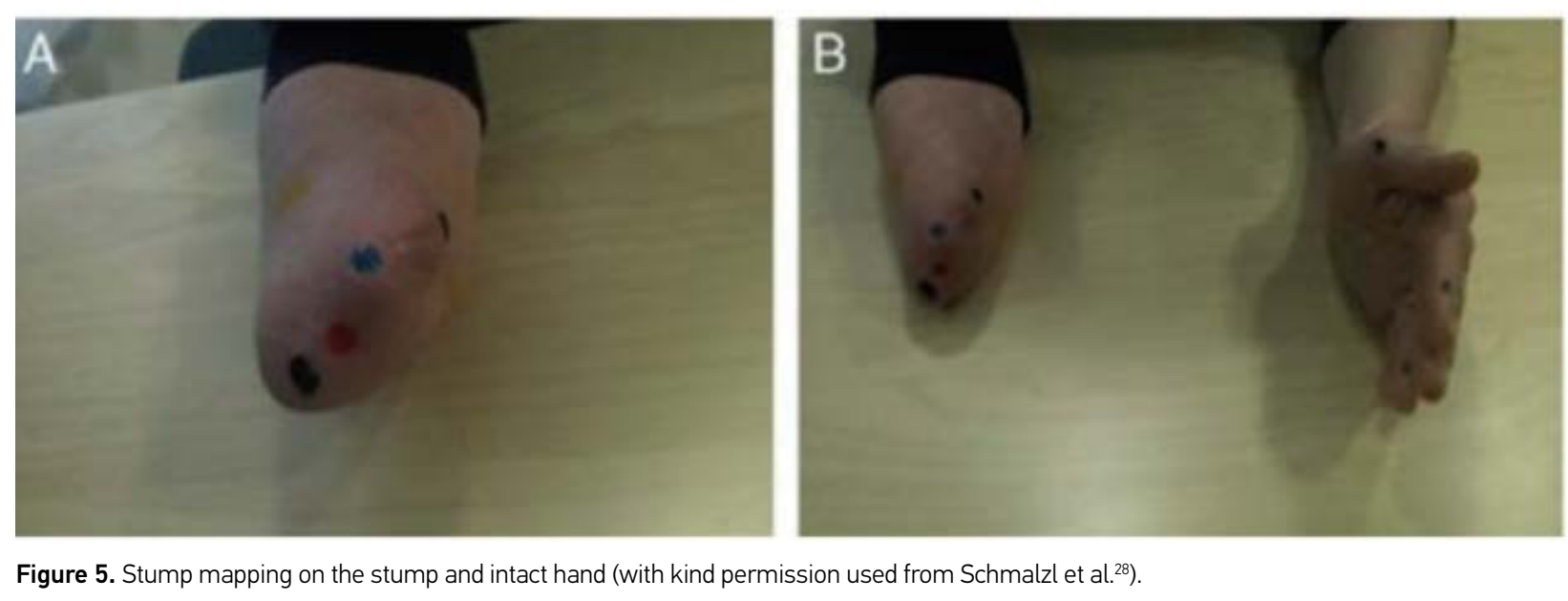

Figure 5. Stump mapping on the stump and intact hand (with kind permission used from Schmalzl et al.?. 
Chapter 3: First therapy sessions

If trigger points are identified during physical examination these points can be treated prior to mirror therapy in order to positively affect malposition of the phantom limb.

The starting position of the limbs and the mirror has already been described in the first chapter. After the limbs and the mirror have been positioned, patients are asked to focus on the mirror image.

Facilitation of visual Illusion

The aim of the first step is to facilitate the mirror illusion. This can be done by instructing patients to observe the mirror reflection for one to instructed to imagine looking through a window instead of a mirror, to enhance the vividness of the mirror illusion. In addilion, he herapist can use bilateral, synchronous stimulation (e.g. tactile) of the stump and corresponding area of the intact limb taking into account the level of amputation and sensitivity of the stump to further facilitate the mirror illusion. After initial bilateral stimulation, the therapist continues to stimulate the intact limb only at the level of amputation. This procedure can be repeated in different positions of the amputated and intact limb. The first exercises can start when the patient indicates that he perceives the mirror image as the affected limb.

\section{Screening of patient preferences}

In order to create a tailored treatment program for every patient, the individual patient preferences should be evaluated during the first sessions. This is done by completing the following exercise categories and selecting the exercises from each category to which the individual patient is responsive. Only exercises that are perceived as pleasant by the patient and lead to motor and / or sensory sensations in the phantom limb should be selected.

Basic motor exercises without objects

This category includes basic motoric exercises such as flexion-extension movements of toes, ankle or knee. In principle, all degrees of freedom of the joints may be addressed. Most common is to start with movements in pain free areas and then slowly proceeding to the more painful regions of the intact limb. The therapist first demonstrates the chosen movement verbally and visually to the patient, who subsequently imitates the movement with the intact limb.

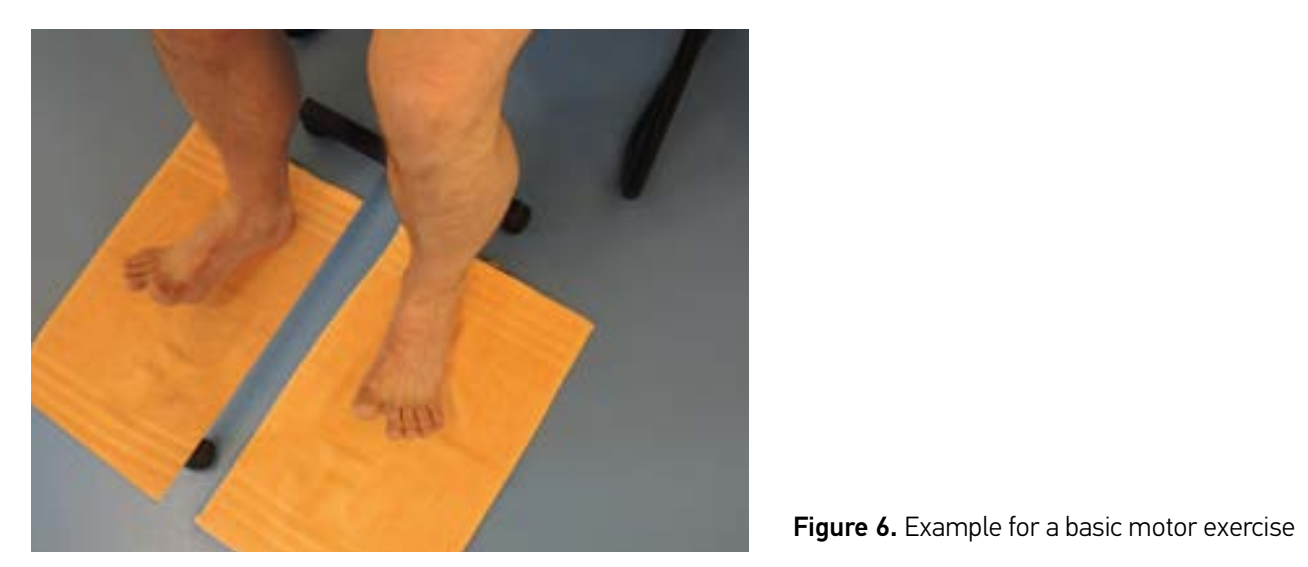

Complexity \& range of movements

The complexity and range of movements usually depends on the patients' ability to voluntary move the phantom limb. If a patient is not able to voluntary move the phantom limb at all, one should start with small movements of the intact limb only, slowly increasing the range and complexity of the movements ' 'shaping'). As soon as the patient feels that he is also able to voluntary move the phantom limb bilateral and complexity of the movements ('shaping). As soon as the patient feels that he is also able to voluntary move the phantom limb bilateral
movements can be initiated. When performing bilateral movements, the natural position of the phantom limb should be used as starting movements can be initiated. When performing bilateral movements, the natural position of the phantom limb should be used as starting
point and the range and complexity of the movements should be adjusted to the capacity of the phantom limb. All movements should be performed below the individual pain threshold.

Sensory Exercises

In this category, many different sensory stimuli can be applied to the intact limb by the therapist or the patient himself (see fig. 7 and chapter 1). Again, one should start with sensory stimuli in pain free areas and then slowly proceed to the more painful regions on the intact limb. With 
regard to the selection of sensory stimuli it is important to assess the individual stimulus preference of the patient; most common is to start with mild stimuli (e.g. soft brushes, heat) before progressing to harder sensory stimuli (e.g. washing up gloves, hard brushes).

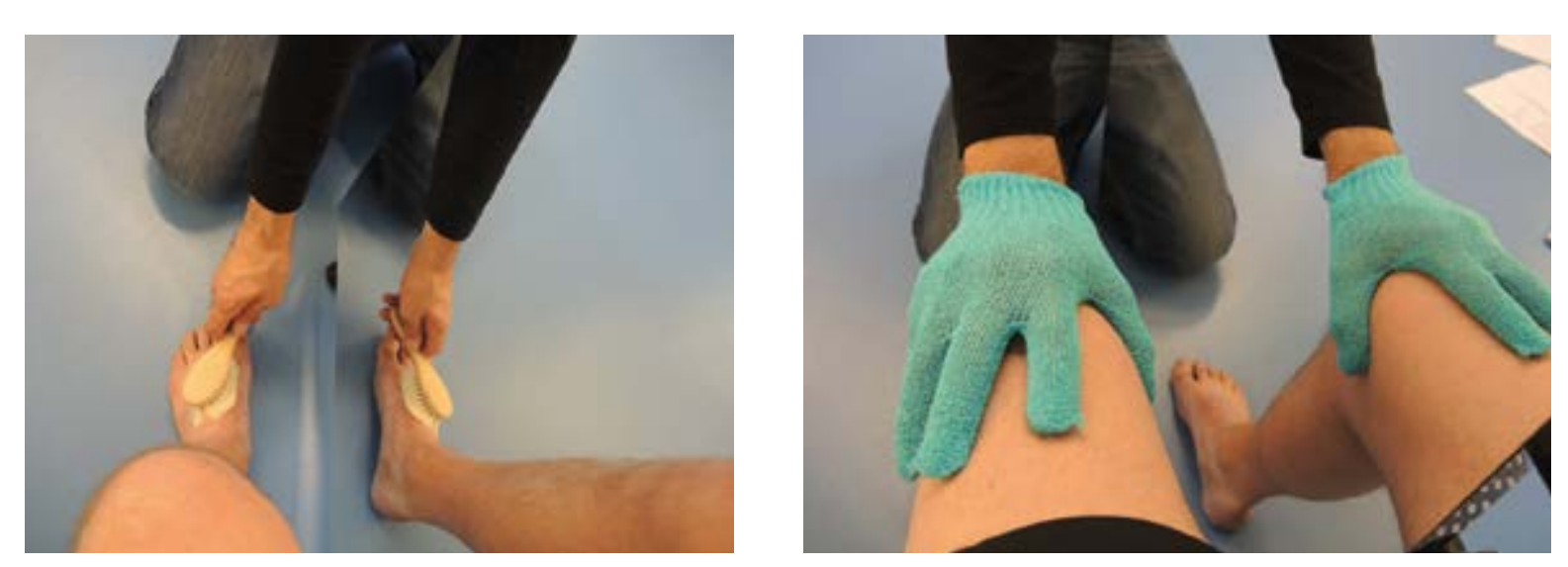

Figure 7. Exercises using sensory stimul

In some patients, a more intense sensory stimulation in the phantom limb can be achieved through first using bilateral, synchronous stimulation of the stump and the corresponding area of the intact limb before applying sensory stimuli to the intact limb only (see 'visual illusion').

In addition, stimulation of the points of the stump map (see chapter 2) can be used. This is done by simultaneously stimulating the points of the stump evoking the strongest referred sensations in the phantom and the corresponding part of the intact limb using different materials (e.g. small brush, cotton wool).

\section{Functional motor exercises with objects}

Following the first sessions (consisting of basic motor exercises and sensory stimulation) additional functional tasks with different objects (e.g. cups, marbles or balls) are integrated into the treatment program. Again, the range and complexity of the movements should be adjusted to the capacity of the phantom limb and patients should pay close attention to slow and gentle movement execution.

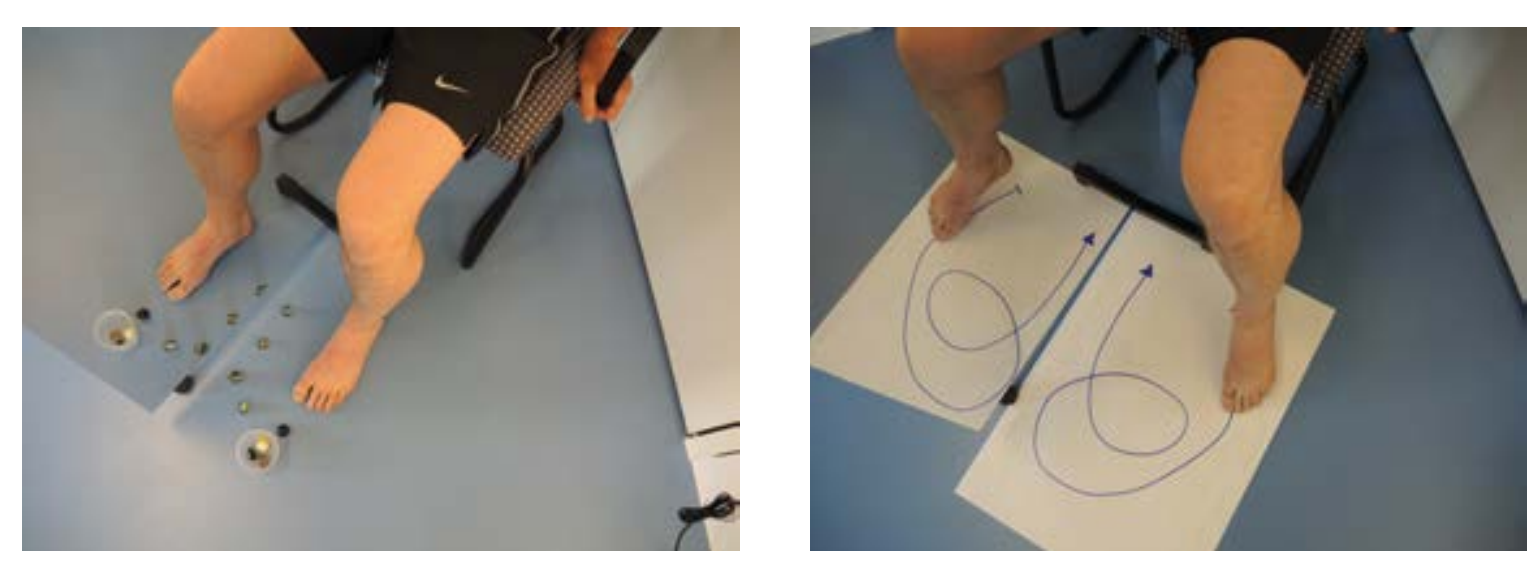

Figure 8. Functional motor exercises using objects

Mental practice

Motor and sensory exercises using the mirror can be complemented by mental practice to enable patients to perform exercises in daily Ire whe Imagined movements of the phantom limb can be preceded by relaxation exercises such as progressive muscle relaxation or mental visualization of different joints of the intact and phantom limb ('body scan').'

Facilitating mental practice using the mirror

Mental practice can be facilitated with or without using the mirror. When using the mirror, the therapist might choose a basic motor exercise that ideally had a positive effect on phantom limb pain. First, the chosen movement is performed with the intact limb in front of the mirror while the patient focuses on the mirror image. Movements of the phantom limb are also executed if the patient is able to voluntary move the phantom without provoking phantom limb pain. The movement is repeated as long as the patient confirms that he sufficiently visualized 
and memorized the movement. Subsequently, the movement is mentally rehearsed with eyes closed using the same speed and range of motion until the mental image of the movement fades out. This phase of mental practice is followed by execution and observation of the movement in front of the mirror (see above). Again, the patient performs the movement as long as he confirms that he sufficiently visualized and memorized the movement. Then movement execution and observation is followed by mental practice of the movement. These phases of movement execution, observation and mental practice alternate each other up to ten times depending on the cognitive abilities of the patient. It is recommended to start with movements the patient is already familiar with (e.g. from mirror therapy or work, sports / hobby) when applying mental practice, as these movements are easier to learn. Functional motor exercises with objects or exercises using sensory stimulation can also be used according to the same principle described above. However in most cases mental practice of these tasks is more difficult.

Facilitating mental practice without the mirror

Mental practice can also be facilitated without the mirror. Patients are comfortably seated on a chair or in bed with their eyes closed. As described above, mental practice of the phantom limb can be preceded by relaxation exercises such as progressive muscle relaxation or mental visualization of different joints of the intact and phantom limb ('body scan'). Patients can focus on sensations from any part of their body, starting with the intact limb before progressing to the residual limb, phantom and the location of phantom limb pain. Patients should body, starting with the intact limb before progressing to the residual limb, phantom and the location of phantom limb pain. Patients should
verbally describe the felt position and other perceptions such as heat or cold in the different parts of the body. Next, patients can be instructed to imagine slow and gentle movements and sensations in the phantom limb.

Perspective of Imagination

Most patients use the first-person-perspective during mental practice, similar to the perspective they already know from observing the mirror image. Some patients prefer the third-person-perspective, as if they observe themselves or others, from a distance' while performing the movement. When performing mental practice visual as well as kinesthetic information can be used to facilitate the vividness of imagery. ${ }^{2,136}$
Limb laterality recognition training

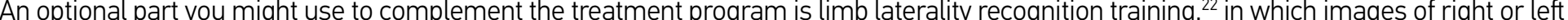
feet are shown in different postures and angles on a screen (fig. 9). These images have to be identified by the patient whether being a left or right limb. In general, one starts with three series of 30 pictures each, slowly increasing the number and complexity of the images shown. Limb laterality recognition training is available on DVD for PC (Physiofun Left Right Training, Kaasa health, Germany) as well as mobile application for iPad ${ }^{\oplus}$ and iPhone ${ }^{\oplus}$ (Limbs by Dr. Becker, Kaasa health, Germany).

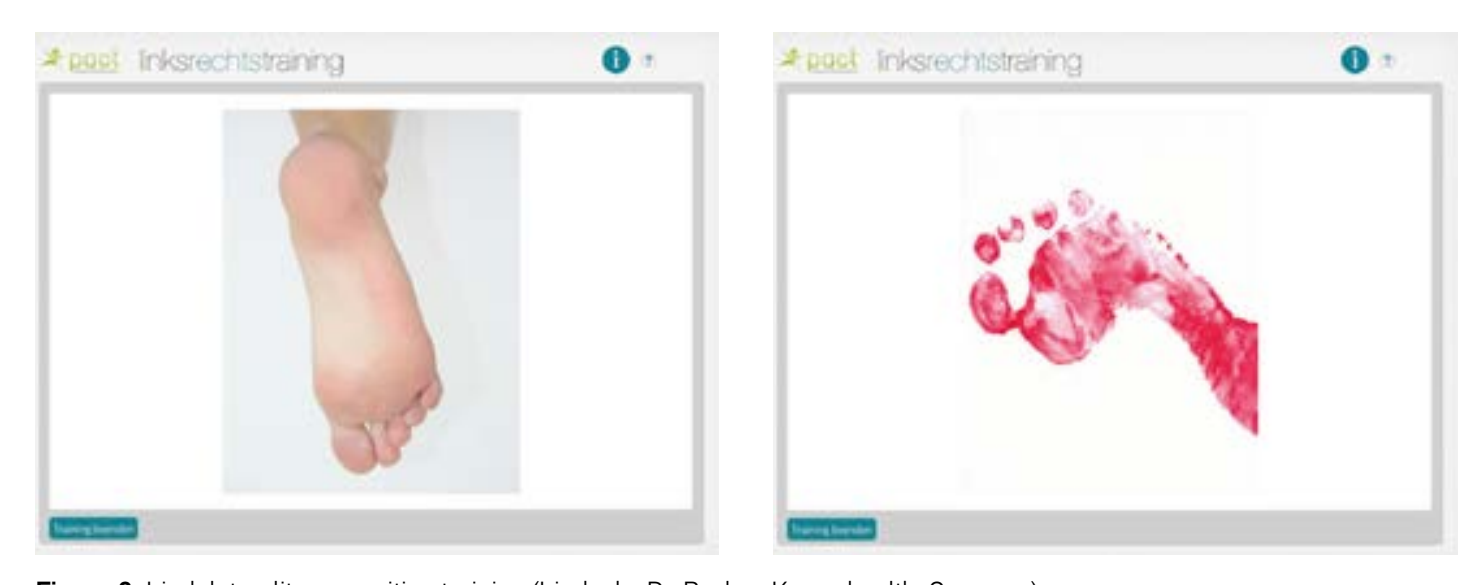

Figure 9. Limb laterality recognition training (Limbs by Dr. Becker, Kaasa health, Germany) 
Chapter 4: Developing a tailored treatment program

Different exercises from the categories described above should be selected according to the individual preferences of the patient in order to create a tailored treatment program. An example of an individual treatment program is given in table 1. This treatment program should always include motor exercises as well exercises using sensory stimuli. However, the emphasis can be shifted to either motor or sensory exercises depending on the patient's preferences (e.g. $70 \%$ sensory and 30\% motor exercises). Furthermore, it is recommended to integrate mental practice as well, in order to enable mobile self-management of patients in daily life when no mirror is available. The tailored treatment program can subsequently be deepened and varied in the following sessions and unsupervised training should gradually be increased.

Table 1. Example of a tailored treatment program using mirror therapy*

\begin{tabular}{|c|c|}
\hline & Exercise \\
\hline Basic motor exercises & $\begin{array}{l}\text { - Rolling foot from heel to toe } \\
\text { - Rotating the foot } \\
\text { - Fexioion-Extension of toes }\end{array}$ \\
\hline Sensory exercises & 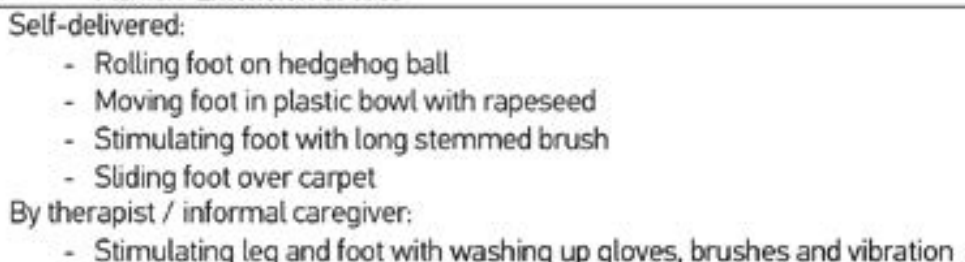 \\
\hline $\begin{array}{l}\text { Functional exercises with } \\
\text { objects }\end{array}$ & $\begin{array}{l}\text { - Putting marbles with the toes in abowl } \\
\text { - Writing numbers with the foot in the air } \\
\text { - Sorting playing cards with the foot }\end{array}$ \\
\hline Mental practice & $\begin{array}{l}\text { - Flexion-Extension of toes } \\
\text { - Rotating the foot } \\
\text { Putting marbles with the toes in a bowl }\end{array}$ \\
\hline
\end{tabular}

\section{Chapter 5: Facilitating unsupervised training}

As soon as possible, patients should be instructed to perform unguided training in order to increase the intensity of the training. Once patients have understood the exercises and are able to perform mirror therapy without the guidance of a therapist, self-directed treatment should ent $3-4$ sessions (after the screening phase of patient preferences is ended). In order to facilitate unguided mirror therapy, it is useful to give written instructions (information sheet) and to ask patients to keep a log on their progress. An example of a mirror therapy log is given below (appendix 2). In addition, providing the patient with the required exercise materials until he has purchased the materials himself facilitates unsupervised training. However, it is useful to enable face-to-face contact with the therapist on the patient's request during the phase of unsupervised training.

\section{When to stop mirror therapy?}

A minimum frequency of ten sessions over a period of four weeks mirror therapy should be performed in order to evaluate possible effects of the treatment. The total duration of the treatment depends on how long improvements in pain or other outcomes are perceived by the individual patient and / or the therapist or to which extend the patient thinks that the treatment is beneficial or necessary to achieve sustainable effects. The treatment should be stopped in case of persistent negative side effects or if unguided training only is sufficient. 


\section{REFERENCES}

Rothgangel AS, Braun S, Schulz RJ, et al. The PACT trial: PAtient Centered Telerehabilitation: effectiveness of software-supported and traditional mirror therapy in patients with phantom limb pain following lower limb amputation: protocol of a multicentre randomised controlled trial. J Physiother. 2015; 61: 42; discussion

2. Sackett DL, Rosenberg WM, Gray JA, Haynes RB and Richardson WS. Evidence based medicine: what it is and what it isn't. BMJ. 1996; 312: 71-2.

Ephraim PL, Wegener ST, Mackenzie EJ, Dillingham TR and Pezzin LE. Phantom pain, residual limb pain . and back pain in amputees: results of a national survey. Arch Phys Med Rehabil. 2005; 86: 1910-9, Kern U, Busch V, Rockland M, Kohl M and Birklein F. Prevalence and risk factors of phantom limb pain and phantom limb sensations in Germany. A nationwide field survey]. Schmerz. 2009; 23: 479-88. Kooijman CM, Dijkstra PU, Geertzen JH, Elzinga A and van der Schans CP. Phantom pain and phantom

sensations in upper limb amputees: an epidemiological study. Pain. 2000; 87: 33-41.
Richardson C, Glenn S, Nurmikko T and Horgan M. Incidence of phantom phenomena including phantom limb pain 6 months after major lower limb amputation in patients with peripheral vascular disease. Clin $J$ Pain. 2006; 22: 353-8.

Bosmans JC, Suurmeijer TP, Hulsink M, van der Schans CP, Geertzen JH and Dijkstra PU. Amputation, phantom pain and subjective well-being: a qualitative study. Int J Rehabil Res. 2007; 30: 1-8.

Hanley MA, Ehde DM, Jensen M, Czerniecki J, Smith DG and Robinson LR. Chronic pain associated with upper-limb loss. Am J Phys Med Rehabil. 2009; 88: 742-51; quiz 52, 79.

Sinha $R$, van den Heuvel WJ and Arokiasamy P. Factors affecting quality of life in lower limb amputees. Prosthet Orthot Int. 2011; 35: 90-6.

10. van der Schans CP, Geertzen JH, Schoppen T and Dijkstra PU. Phantom pain and health-related quality of life in lower limb amputees. J Pain Symptom Manage. 2002; 24: 429-36.

11. Griffin SC and Tsao JW. A mechanism-based classification of phantom limb pain. Pain. 2014; 155: 2236-42.
12. Abbass K. Efficacy of gabapentin for treatment of adults with phantom limb pain. Ann Pharmacother. 2012; 46: 1707-11.

13. Barrett AM, Lucero MA, Le T, Robinson RL, Dworkin RH and Chappell AS. Epidemiology, public health burden, and treatment of diabetic peripheral neuropathic pain: a review. Pain Med. 2007; 8 Suppl 2: S50-62.

14. Alviar MJ, Hale T and Dungca M. Pharmacologic interventions for treating phantom limb pain. Cochrane Database Syst Rev. 2011: CD006380.

15. Birbaumer N, Lutzenberger $W$, Montoya $P$, et al. Effects of regional anesthesia on phantom limb pain are mirrored in changes in cortical reorganization. J Neurosci. 1997; 17: 5503-8.

16. Flor $\mathrm{H}$, Elbert $\mathrm{T}$, Knecht $\mathrm{S}$, et al. Phantom-limb pain as a perceptual correlate of cortical reorganization following arm amputation. Nature. 1995 ; 375: 482-4.

17. Karl A, Birbaumer N, Lutzenberger $W$, Cohen $L G$ and Flor $H$. Reorganization of motor and somatosensory cortex in upper extremity amputees with phantom limb pain. J Neurosci. 2001; 21: 3609-18.

18. Koppelstaetter $F$, Siedentopf $\mathrm{CM}$, Rhomberg P, et al. Functional magnetic resonance imaging before motor cortex stimulation for phantom limb pain]. Nervenarzt. 2007; 78: 1435-9.

19. Flor H, Nikolajsen $L$ and Staehelin Jensen T. Phantom limb pain: a case of maladaptive CNS plasticity? Nat Rev Neurosci. 2006; 7: 873-81.

20. Chan BL, Witt R, Charrow AP, et al. Mirror therapy for phantom limb pain. N Engl J Med. 2007; 357: 2206-7. 21. Maclver K, Lloyd DM, Kelly S, Roberts $N$ and Nurmikko T. Phantom limb pain, cortical reorganization and the therapeutic effect of mental imagery. Brain. 2008; 131: 2181-91.

22. Moseley GL. Graded motor imagery for pathologic pain: a randomized controlled trial. Neurology. 2006; 67:2129-34.

23. Foell J, Bekrater-Bodmann R, Diers M and Flor H. Mirror therapy for phantom limb pain: brain changes and the role of body representation. Eur J Pain. 2014; 18: 729-39.

24. Rothgangel AS, Braun SM, Beurskens AJ, Seitz RJ and Wade DT. The clinical aspects of mirror therapy in rehabilitation: a systematic review of the literature. Int J Rehabil Res. 2011; 34: 1-13. 
25. Darnall BD. Self-delivered home-based mirror therapy for lower limb phantom pain. Am J Phys Med Rehabil. 2009; 88: 78-81.

MacLachlan M, McDonald D and Waloch J. Mirror treatment of lower limb phantom pain: a case study. Macsachlan M, MCDonald D and

27. Darnall BD and Li H. Home-based self-delivered mirror therapy for phantom pain: a pilot study. J Rehabil Darnall BD and LiH.

28. Schmalz L, Ragno C and Ehrsson HH. An alternative to traditional mirror therapy: illusory touch can reduce phantom pain when illusory movement does not. Clin J Pain. 2013; 29: e10-8.

29. de Vries C, Hagenaars L, Kiers H, Schmitt M. 2006. The professional profile of the physical therapist. Amersfoort: Royal Dutch Society for Physical Therapy.

30. Casale R, Damiani C and Rosati V. Mirror Therapy in the Rehabilitation of Lower-Limb Amputation: Are There Any Contraindications? Am J Phys Med Rehabil. 2009; 88: 837-42.

31. Hagenberg $A$ and Carpenter C. Mirror visual feedback for phantom pain: international experience on modalities and adverse effects discussed by an expert panel: a delphi study. PM R. 2014; 6: 708-15.

Diers M. Christmann C, Koeppe C. Ruf M and Flor H. Mirrored imagined and executed movements differentially activate sensorimotor cortex in amputees with and without phantom limb pain Pain. 2010; differentially activate sensorimotor con ex in

33. Lotze M, Flor H, Grodd W, Larbig W and Birbaumer N. Phantom movements and pain. An fMRI study in upper limb amputees. Brain. 2001; 124: 2268-77.

McCabe C. Mirror visual feedback therapy. A practical approach. J Hand Ther. 2011; 24: 170-8; quiz 9 . Winter C, Fritsche K, Karl A, et al. The phantom and stump phenomena interview (PSPI)]. Schmerz. 2001; 15: 172-8.

36. Beaumont G, Mercier C, Michon PE, Malouin F and Jackson PL. Decreasing phantom limb pain through observation of action and imagery: a case series. Pain Med. 2011; 12: 289-99. 
APPENDIX CLINICAL FRAMEWORK MIRROR THERAPY

APPENDIX 1 Patient information sheet for mirror therapy

APPENDIX 2 Mirror therapy Log

APPENDIX 3 Clinical flow chart mirror therapy 
Mirror therapy - important recommendations for patients (information sheet)

- Consult your therapists or doctor when you are using mirror therapy and ask for feedback when you are unsure if you are performing the exercises correctly.

- The illusion in the mirror should be as realistic as possible. Therefore - if it confuses you - visible marks on the intact limb such as jewellery, scars or tattoos should be covered or taken off.

- Important: Adjust the intensity of the exercises with regard to speed, range of motion and complexity depending on unpleasant sensations (e.g. pain) you might be experiencing. You may also want to vary exercises or change to another kind of exercise. You should always practice below your pain threshold Neither during practice nor afterwards should you experience more pain than usual.

- Mirror therapy is more likely to be successful if you practice regularly. You should therefore try to perform your mirror therapy exercises at least once a day for at least 10 minutes.

- When starting with mirror therapy you should perform your exercises in a quiet surrounding to avoid distraction as much as possible.

- The amputated limb should be completely hidden by the mirror while you are practising.
- It is essential that you concentrate on the limb in the mirror during the entire time you are practising. Try to imagine that the reflection of your intact limb in the mirror actually is your affected limb. In most cases the exercises will be more beneficial the more vivid or realistic the mirror illusion is

- Try to avoid looking at your intact limb during practice.

- Perform the movements slowly and with focus. The longer the symptoms have been existing, the slower you should proceed.

- You might want to add extra exercises yourself and / or vary existing exercises. You should always feel comfortable when performing the exercises.

- In most cases the exercises will be more beneficial the more and continuously you practise. Try to practise at least once daily with a minimum duration of 10-15 minutes.

- Use a log to record your exercise progress: How often and for how long have you performed which exercises? What effect does the mirror therapy have on your complaints? Are there any unintended side effects? Have you taken less or extra medication? 
APPENDIX 2. Mirror therapy log example

Name patie

Mirror therapy log

$$
\text { Week }
$$

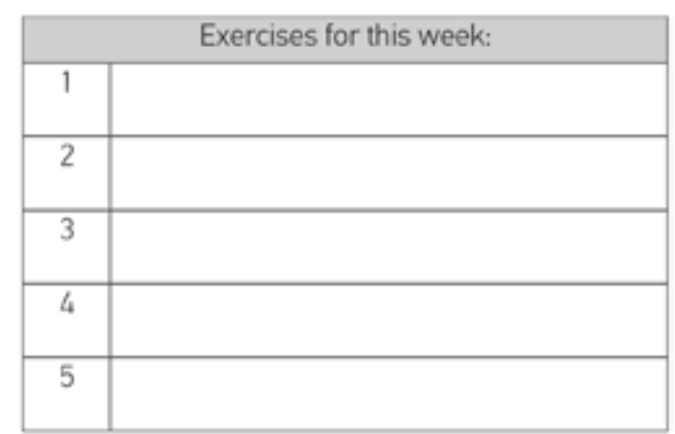

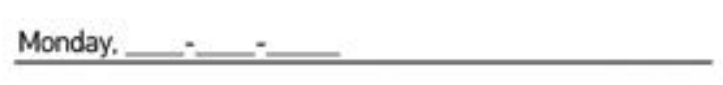

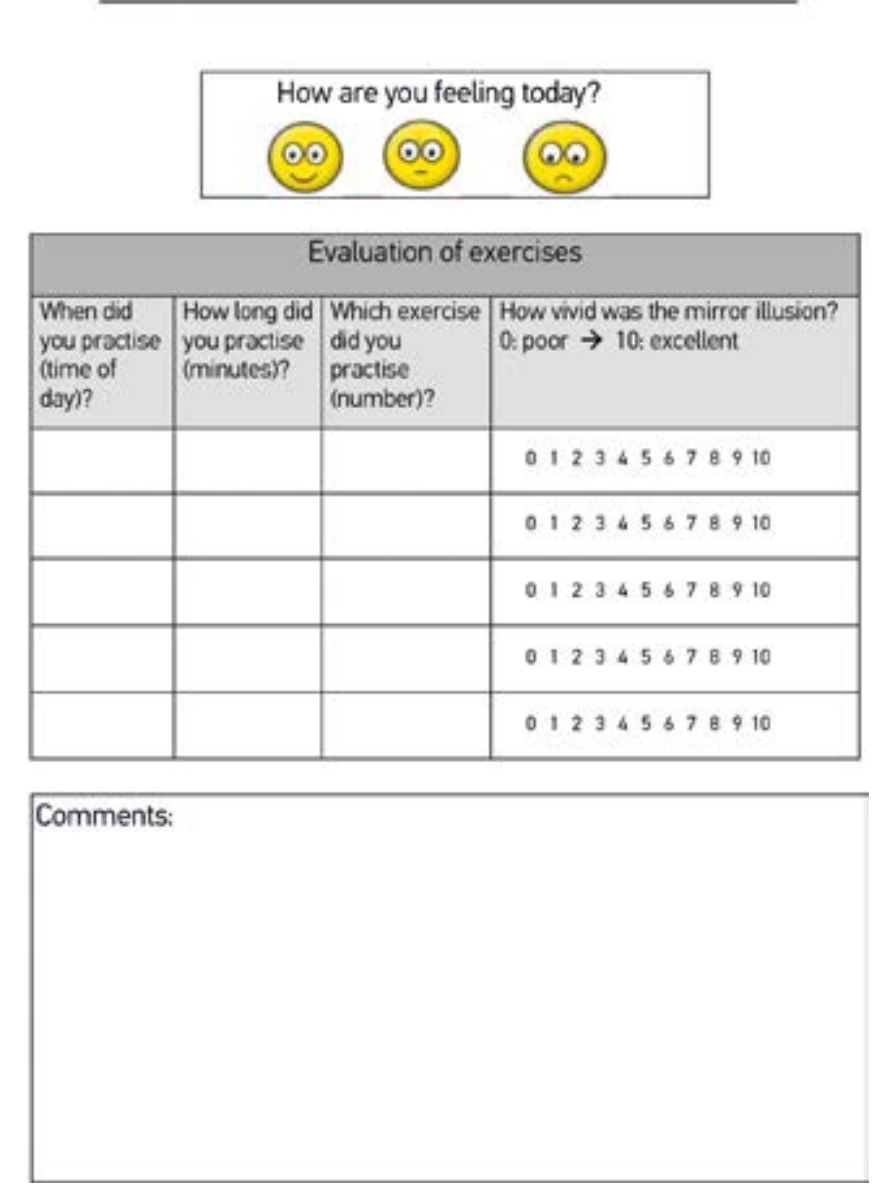

APPENDIX 3. Clinical flow chart mirror therapy

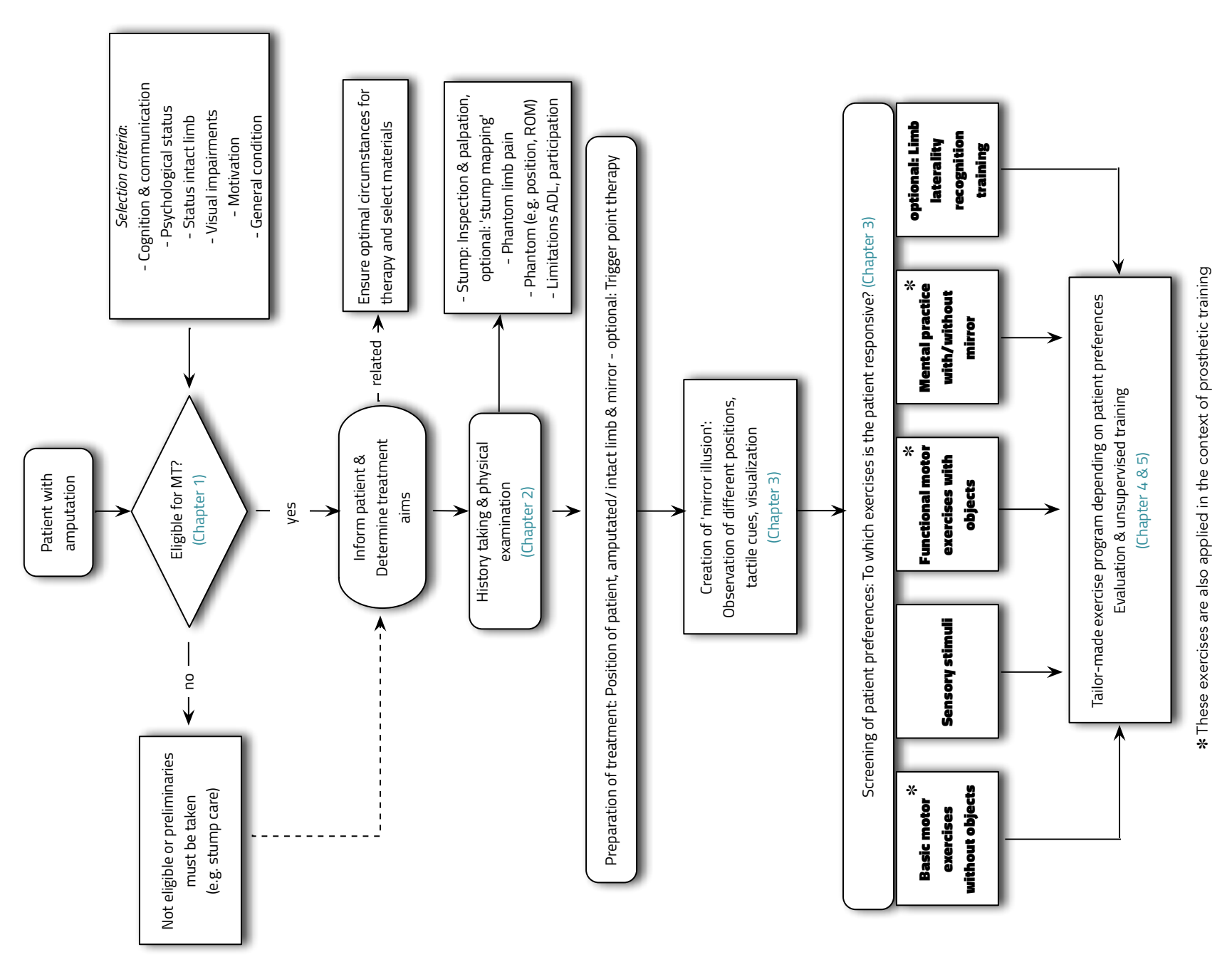





\section{ABSTRACT}

\section{BACKGROUND}

Phantom limb pain is a frequent and persistent problem following amputation. Achieving sustainable favorable effects on phantom limb pain requires therapeutic interventions such as mirror therapy that target maladaptive neuroplastic changes in the central nervous ation to support long-term self-managem

\section{OBJECTIVE}

The main aim of this study was to describe the user-centered approach that guided the design and development of a telerehabilitation platform for patients with phantom limb pain. We addressed 3 research questions: (1) Which requirements are defined by patients and therapists for the content and functions of a telerehabilitation platform and how can these requirements be prioritized to develop a firs prototype of the platform? (2) How can the user interface of the telerehabilitation platform be designed so as to match the predefined with pha rem limb routine care and how can the platform be redesigned based on their feedback to achieve a high-fidelity prototype?

\section{METHODS}

The telerehabilitation platform was developed using an iterative user-centered design process. In the first phase, a questionnaire followed by a semistructured interview was used to identify the user requirements of both the patients and their physical and occupational therapists, which were then prioritized using a decision matrix. The second phase involved designing the interface of the telerehabilitation platform using design sketches, wireframes, and interface mock-ups to develop a low-fidelity prototype. Heuristic evaluation resulted in a medimW

RESULTS

A total of 7 categories of patient requirements were identified: monitoring, exercise programs, communication, settings, background information, log-in, and general requirements. One additional category emerged for therapists: patient management. Based on these requirements, patient and therapist interfaces for the telerehabilitation platform were developed and redesigned by the software development team in an iterative process, addressing the usability problems that were reported by the users during 4 weeks of field testing in routine care.

\section{CONCLUSIONS}

Our findings underline the importance of involving the users and other stakeholders early and continuously in an iterative design process, as well as the need for clear criteria to identify critical user requirements. A decision matrix is presented that incorporates the views of various stakeholders in systematically rating and prioritizing user requirements. The findings and lessons learned might help health care providers, researchers, soffware designers, and other stakeholders in designing and evaluating new teletreatments, and hopefully increase the likelihood of user acceptance. 


\section{INTRODUCTION}

Phantom limb pain is a frequent and persistent problem following amputation. Despite many pharmacological and nonpharmacological Interventions, up to $80 \%$ of patients still suffer from phantom limb pain many years after the amputation..$^{-3} \mathrm{According}$ to a recent trial, ${ }^{3} 63 \%$ of a sample of 3234 amputees with an average time since amputation of 33 years, were still suffering from phantom limb pain. These data illustrate the chronic nature of this disorder, which is accompanied and maintained by a wide range of changes in the peripheral' and central nervous system. ${ }^{5}$ Achieving sustainable favorable effects on phantom limb pain requires therapeutic interventions such as mirror therapy ${ }^{6}$ that target these maladaptive neuroplastic changes in the central nervous system.

Two recent systematic reviews 78 reported that despite the potential merits of mirror therapy, the quality of evidence for patients with phantom limb pain is still low and a detailed description of how to deliver the intervention is lacking Therefore, we recently developed an evidence-based clinical framework for mirror therapy for patients with phantom limb pain? that is currently being tested for effectiveness in a multicenter randomized controlled trial $1{ }^{10}$ Given the chronic nature of phantom limb pain continuous training with at least one session a day over a period of several weeks to months seems to be needed to achieve sustainable treatment effects. ${ }^{7}$ However, resources in clinical practice (the desired training intensity Unfortunately, patients' adherence to unsupervised training is generally poor. 11 i mplying the need for effective strategies to support long-term self-management by patients with phantom limb pain.

the use of information and communication technology such as telerehabilitation, which allows patients

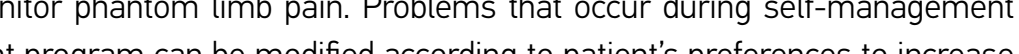
long-term adherence to self-administered exercises. 12,13 The use of telerehabilitation has been shown to enhance treatment intensity ${ }^{14}$ selfefficacy, 15,16 and compliance with self-administered exercises, that in turn correlates positively with the effects of the intervention ${ }^{17}$ Moreover: the implementation of these potential time- and cost-saving strategies might lead to increased accessibility and enhanced continuity of care 18 $^{8}$ Data regarding the effects of telerehabilitation in patients with phantom limb pain is sparse In a recent study 19 a teletreatment for 2 patients with phantom limb pain using mirror therapy was described This teletreatment solely consisted of email instructions by a physician on how to deliver self admistered miror therapy. Both the patients reported complete recory from phantom limb pain after daily execises for teletreatment was developed and whether the end users were involved during the design of the system.

To facilitate user acceptance, such teletreatments have to be easy to use, ${ }^{20}$ match the requirements and preferences of the end users ${ }^{21}$ and $\mathrm{fit}$ in their personal context. ${ }^{22}$ This is supported by theoretical models such as the technology acceptance model (TAM) ${ }^{23.24}$ and the unified theory of acceptance and use of technology (UTAUT) 25.26 that assume that user acceptance and the intention to use a telemedicine service is predicted by factors such as perceived usefulness, perceived ease of use, as well as intrinsic motivation and social influence. Therefore, it is essential to involve the end users in the design and development of any new telerehabilitation platform. In the PAtient Centered Telerehabilitation (PACT) project, ${ }^{10}$ we developed an innovative mobile telerehabilitation platform using mirror therapy for patients with phantom limb pain following lower limb amputation. Patients and physical and occupational therapists were involved throughout the entire platform development process.

The aim of this study was to describe the user-centered approach that guided the design and development of the telerehabilitation platform.

The following research questions were addressed:

Which requirements are defined by patients with phantom limb pain following lower limb amputation and the occupational and physical

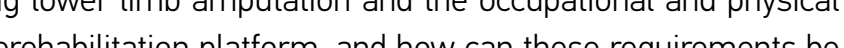
prioritized to develop a first prototype of the platform?

How do patients with phantom limb pain and their treating therapists judge the usability of the medium-fidelity prototype of the telerehabilitation platform in routine care, and how can the platform be redesigned based on their feedback to achieve a high-fidelity prototype?

Our description of this process and the lessons learned along the way aims to offer insights into the complexity of the user-centered design the daily routines of the users. Our findings might help health care providers, researchers, software designers, and other stakeholders in designing and evaluating new teletreatments. 
The framework to improve the uptake and impact of eHealth technologies ${ }^{27}$ and the method of agile soffware development ${ }^{28}$ were used in an iterative user-centered design process to develop the telerehabilitation platform in 3 phases (Figure 1).

Important topics that are mentioned in the framework of van Gemert-Pinen ${ }^{27}$ such as a participatory development and design approach, value specification through identification of user requirements, as well as persuasive design techniques and continuous evaluation cycles were also addressed in this study.

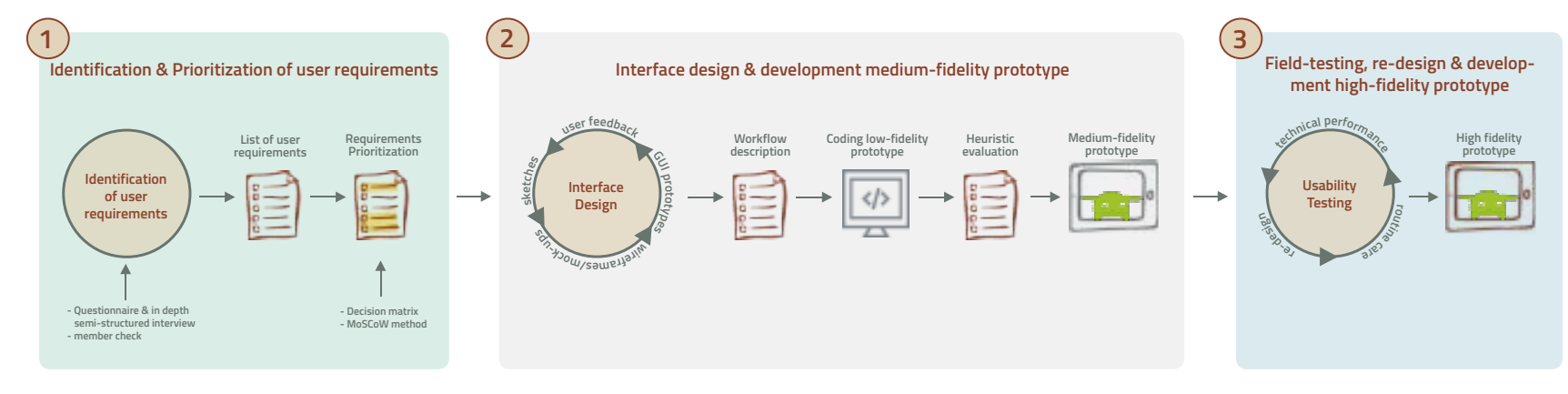

Figure 1. Overview of the 3 phases and methods used throughout the user-centered approach.

Recruitment of Patients

We used purposive sampling to achieve a wide range of patient characteristics (eg, age, gender, reason for amputation, time since amputation) to obtain a rich date collection The principl investigator (AR) identifed eligible patients by co and orthopedic technicins and placing Web-based advertisements in Germany In addition, the therapists who participted in the interviews selected patients whom they had treated in the past or whom they were currently treating. Adult patients with unilateral amputation of the lower limb and suffcient coonitive and lingristic capacities to participate in a 1 hour intervew were included In addition patients needed to have sufficient experience lin using minor theray, which tos defined as having attended at months. Selection of patients was based on the judgment of the recruiting principal investigator or therapists.

Recruitment of Therapists

The principal investigator identified physical and occupational therapists by email or phone viaexisting networks in Germany. The professionals needed to have sufficient experience in using mirror therapy for patients with phantom limb pain, which was defined as having treated at least three patients during the past 12 months. Again, we tried to include a wide range of therapist characteristics (eg profession, age, experience, work setting) to obtain a rich data collection.

Phase 1: Identification and Prioritization of User Requirements (Research Question 1)

In the first phase, a questionnaire followed by a semistructured interview was used to identify the user requirements of both the patients suffering from phantom limb pain and the physical and occupationat therapists the reported requrem requir w decision matrix.

Collection and Analysis of Dato

We developed a structured questionnaire for patients and therapists that contained questions on patient and therapist characteristics such as level and side of ampution a case description of a patent with phon and 3 general items regarding the content and functions of the platform (es "which information, content or functions should be included in the telerehabilitation platform enabling tailored support of your patients regarding self-delivered exercises?") In addition. 3 theranist respectively 6 patient questions regarding user acceptance, barriers and facilitators, and context of use were included (eg which aspects are relevant to increase patient and therapist acceptance of the telerehabilitation platform?) The questionnaire was checked on integrity and comprehensibility by 5 therapists and 1 patient representative. After some minor text revisions and ffter participants gave informed consent the principal investigator sent the questionnaire by email to all patients and therapists who were to participate in the interviews 2 weeks before the interview took place The completed a bestionnire was to be returned at least on day before the interview. The principet investigator checked the dat recarding the telerehabilitation platform before the interview took place to prepare for the interview and refined in-depth questions on the various topics.

All interviews were conducted by the principal investigator in a quiet room at the patient's home or at the professional's clinic. The interviews 
lasted approximately 1 hour and were digitally audio-taped and subsequently transcribed using the $f 4$ software (audiotranskription, Marburg Germany) In addition the principal investigator took field notes after each interview describing the context of the interview Atter 6 interviews had been transcribed, the principal investigator used data analysis to check which topics emerged, and recruited additional patients and therapists until data saturation was achieved.

The data regarding patient and therapist characteristics were extracted from the questionnaires and displayed in a frequency table. Data regarding the topics relating to the telerehabilitation platform were analyzed using directed content analysis. ${ }^{29}$ The initial coding scheme was based on the topics of the questionnaire. This scheme was extended as new topics emerged from the data analysis. After each interview, the data were summarized by topic in a table and were subsecuently sent to the interviewee, who was asked to check the data for integrity and correctness (member check). The interviewees returned the adjusted summary of the data to the principal investigator by email A sample of 2 patient and 2 therapist interviews was independently analyzed by another researcher (SB) and the results were discussed with the requirements regarding each topic were specified in a table to create a requirements catalog

Requirements Prioritization

The user requirements were subsequently prioritized to decide which requirements from the requirements catalog were critical to include in the first prototype of the telerehablitation platform. We developed a decision various stakeholders in the project (patients, therapists, researchers, and software development team, see also Table 2)

Best available evidence: A systematic literature review regarding the clinical framework of mirror therapy for patients with phantom limb

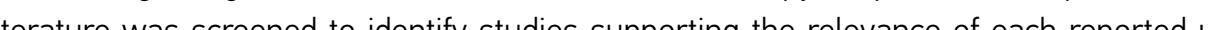
pain was conducted in a preliminary stage. requirement.

Technical complexity: Members of the software development team were also asked to rate the different requirements in order to determine the technical complexity of each requirement. They were asked whether implementation of each requirement would be time-consuming or expensive. The technical complexity of each requirement was assessed by 3 engineers from the software development team (Kaasa health, Düsseldorf, Germany) using an 11 -point numeric rating scale (0=very low, $10=$ very high complexity).

Importance of requirements: The importance of the requirement was primarily defined by the number of respondents who mentioned the requirement and whether or not there was agreement between patients and therapists (eg. the more respondents mentioned the same requirement, the more important the requirement). However, an exception was made for requirements that were only mentioned by a minority of users but were nevertheless regarded as important by the research team that rated the priority of requirements.

Based on these criteria, 3 members of the research team (RS, AJB, AR) rated the priority of each user requirement independently on 作 rating scale according to the MoSCoW prioritization method (1=Must have, $2=$ Should have, $3=$ Could have, $4=$ Won't have at this time. ${ }^{30}$

Only requirements that were scored as priority stage 1 or 2 by at least two of the 3 raters were defined as critical for the first prototype of the telerehabilitation platform.

Phase 2: Interface Design and Development of Medium-Fidelity Prototype (Research Question 2)

Based on the critical user requirements defined in phase 1, the interface of the telerehabilitation platform was designed using design sketches, wireframes, and interface mock-ups (Balsamiq Mockups, version 2210, Balsamiq Studios, Sacramento). All critical user requirements belonging to 1 specific category were used to build the first design sketches incorporating these requirements. In the next step the interface designer of the software development team converted these mock-ups into graphical user interface (GUI) prototypes. The GUI prototypes were shown in several iterative phases, on screen or paper, to a sample of 6 patients and 5 therapists who had been interviewed in phase 1 , to provide feedback regarding the content and design of the prototypes. Their feedback was summarized and discussed with the interface designer, to refine the GUI prototypes. Evaluation of GUI prototypes continued until the majority (>50\%) of patients and therapists made no further comments, and the finl interface design emerged For each category of user requirements, a workflow description was composed in which the finl GUl was used to illustrate the sequentil steps tobe taken by the users when operating the application. Based on this work description, the source code was programed for each application to develop a low-fidelity prototype of the telerehabilitation platform.

Heuristic Evaluation

The usability of the low-fidelity prototype was tested in a laboratory situation by 3 therapists who had already been involved in phase 1, as

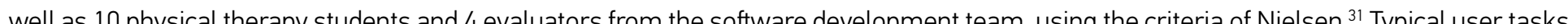
such as logging in and recording a pin score or selecting a tilo

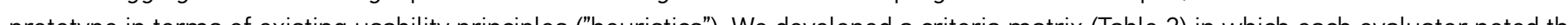
feedback on 
this is a usability problem at all, $5=$ Usability catastrophe) according to the frequency and persistence of the usability problem and its impact on the workflow. ${ }^{32}$ The results of the heuristic evaluation were reported to the software development team, who fixed usability problems with a minimal severity score of 3 to create a medium-fidelity prototype of the telerehabilitation platform.

Table 1. Characteristics of patients participating in the interviews.

Following the heuristic evaluation, the medium-fidelity prototype was tested for usability and technical performance in routine care by 2 physical and 3 occupational therapists who had already taken part in phase 1 and also participated in the multicenter trial. ${ }^{\circ}$ Each therap 2 was asked to select 2 patients with phantom limb pain whom they were currently treating. The participating therapists were trained regarding the content and application of the telerehabilitation plattorm. Subsequently each therapist was asked to instruct patients with phantom limb pain on how to use the telerehabilitation platform before patients were discharged from the rehabilitation center. After discharge, patients and therapists used the telerehabilitation platform for a period of 4 weeks. During this period, the users were encouraged to use various aspect

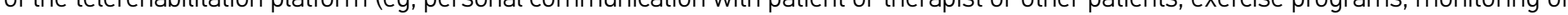
phantom limb pain) and were asked to note any usability problem by means of an in-app feedback system that automatically transferred the user feedback to the solware development team.

In addition, patients and therapists were phoned once a week by the principal investigator to assess usability problems that were not automatically recorded through the in-app feedback system. All usability problems were listed in a standardized bug log and scored by the principal investigator for pilonty (low, medum, high). The technical pefor users reported no more major bugs and a high-fidelity prototype of the telerehabilitation platform had been achieved

Ethical Approval

This study has been approved by the Ethics Committee of the Medical Faculty of Cologne University, Cologne, Germany (approval no. 12-029). RESULTS

Inse 1: Identification and Prioritization of User Requirements (Research Question 1)

In of patients was very heterogeneous as shown in Table 1 .

\begin{tabular}{|c|c|c|c|c|c|c|c|c|}
\hline Patient & $\begin{array}{l}\text { Age } \\
\text { (years) }\end{array}$ & Gender ${ }^{2}$ & Work status & $\begin{array}{l}\text { Time since amputa- } \\
\text { tion (months) }\end{array}$ & $\begin{array}{l}\text { Side of } \\
\text { amputa- } \\
\text { tion }\end{array}$ & $\begin{array}{l}\text { Level of } \\
\text { amputation }\end{array}$ & $\begin{array}{l}\text { Reason for } \\
\text { amputation }\end{array}$ & $\begin{array}{l}\text { Information and communi } \\
\text { cations technology experi- } \\
\text { ence }\end{array}$ \\
\hline 1 & 22 & $\mathrm{~F}$ & Student & 15 & Left & $\Pi^{b}$ & Trauma & High \\
\hline 2 & 49 & M & Part-time & 12 & Right & $\pi$ & Trauma & Medium \\
\hline 3 & 56 & $\mathrm{~F}$ & Retired & 5 & Right & $\pi$ & Vascular & Low \\
\hline 4 & 64 & M & Retired & 116 & Right & $H D^{c}$ & Vascular & High \\
\hline 5 & 49 & $\mathrm{~F}$ & Retired & 27 & Right & HD & Vascular & High \\
\hline 6 & 70 & M & Retired & 36 & Left & $\mathrm{TF}^{\mathrm{d}}$ & Vascular & Low \\
\hline 7 & 39 & $\mathrm{~F}$ & Retired & 39 & Left & HD & Infection & High \\
\hline 8 & 49 & M & Retired & 328 & Right & $H P^{e}$ & Trauma & High \\
\hline 9 & 47 & M & Retired & 35 & Right & TF & Vascular & Medium \\
\hline 10 & 59 & $\mathrm{~F}$ & Full time & 3 & Right & TF & Vascular & Low \\
\hline 11 & 24 & $\mathrm{~F}$ & Student & 45 & Left & $F^{f}$ & Trauma & High \\
\hline
\end{tabular}


The occupational $(n=5)$ and physical $(n=5)$ therapists (age range 23-57 years) had extensive work experience in treating amputees ranging low level, 3 reported a medium, and 4 reported a high level of experience in using information and communication technology.

Requirements Defined by Patients and Therapists

A total of 63 patient requirements and 64 therapist requirements were identified. After the prioritization process, 24 patient requirements and 35 therapist requirements remained that were classified as critical for the first prototype of the telerehabilitation platform (Table 2). Seven categories of patient requirements were identfied: Monitoring (eg. monitoring of phantom pain and self-administered exercises) training programs (eg mirror therapy mental practice) communication (eg text messages, videoconferencing), settings (eg, personal data reminder) background information (eg phantom pain, training programs), and log in and general requirements (eg, privacy, gamification) overview).

We decided to develop a mobile app of the telerehabilitation platform as the majority of the patients and therapists preferred mobile access to the platform in order to be more flexible regarding the time and place of platform use.
Table 2. Prioritization of user requirements using the decision matrix (example shows 4 out of 64 therapist requirements from the category" monitioring")

10 Category 1: Monitoring Deasion criteria

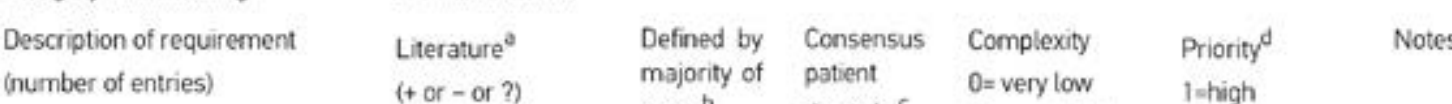

Gumber of entriess)

$\begin{array}{lll}\text { users }^{b} & \text { therapist } & 0=\text { very low } \\ 10=\text { very high } & \text { 1-high }\end{array}$

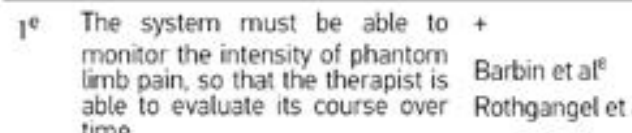
able to
time
$(10 / 10)$ 2 The system has torecord the per-
ceves postion and range of mo-
tion of the phantiom limb (1/10)

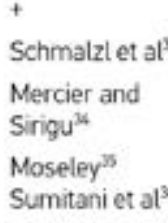

$3^{e}$ The system must enable the Moseley

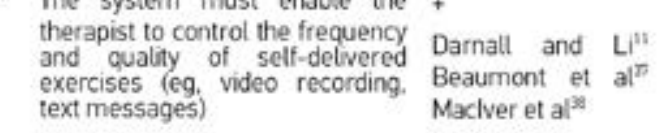

4 The system has to record the per.

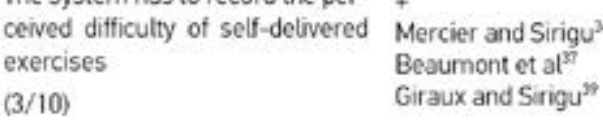


Phase 2: Interface Design and Development of Medium-Fidelity Prototype (Research Question 2)

Based on the 7 categories of user requirements identified, a mobile app was developed for each category. incorporating all user requirements belonging to this category. using an iterative design process. The development process is illustrated in the following section using the example of phantom limb pain monitoring.

Ten patients and all therapists agreed that the telerehabilitation platform should be able to monitor the frequency, duration, type, and intensity of phantom limb pain (Figure 2).

These mock-ups resulted in the first graphical user interface (GUI) prototypes (Figure 3). The feedback from patients and therapists regarding the GUI prototypes showed that 6 patients and 5 therapists required a more compact and comprehensive overview of the most important Whe for monitoring phantom limb pain emerged after 7 iterative rounds with patients and therapists.
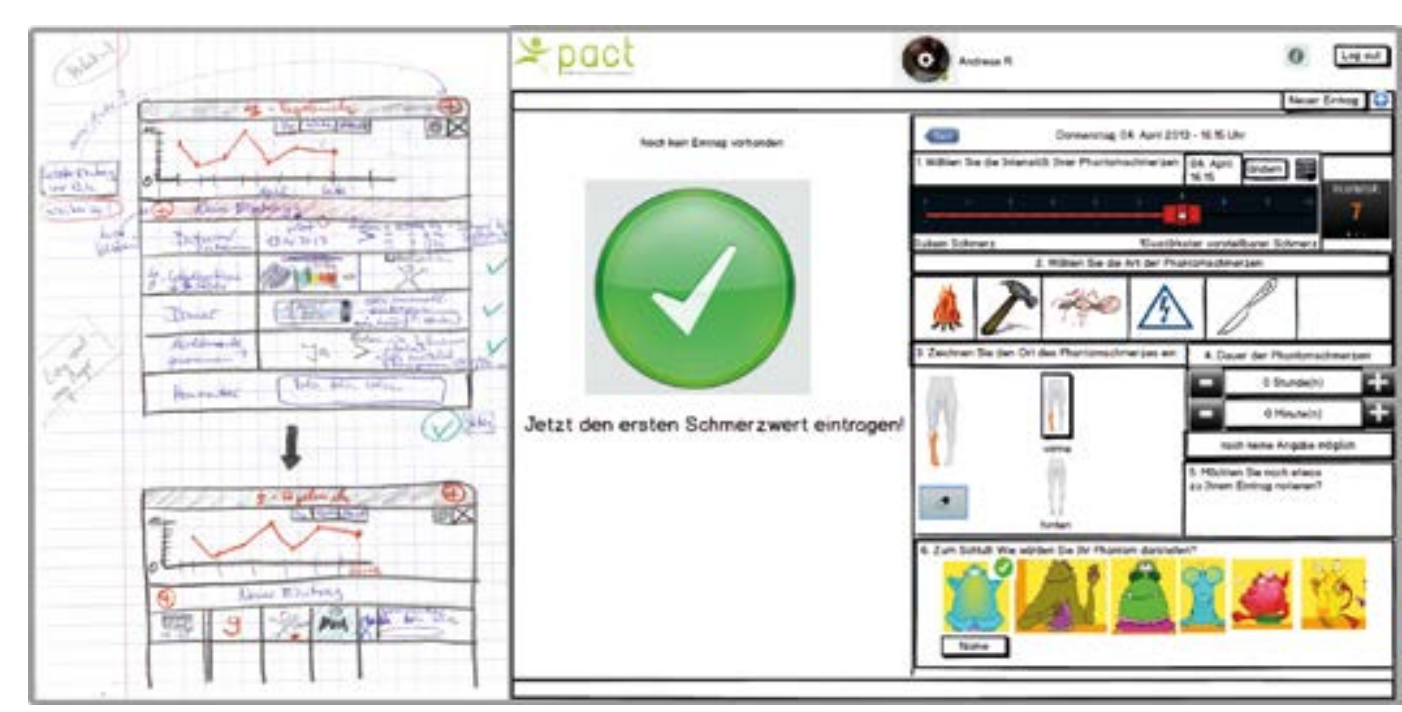

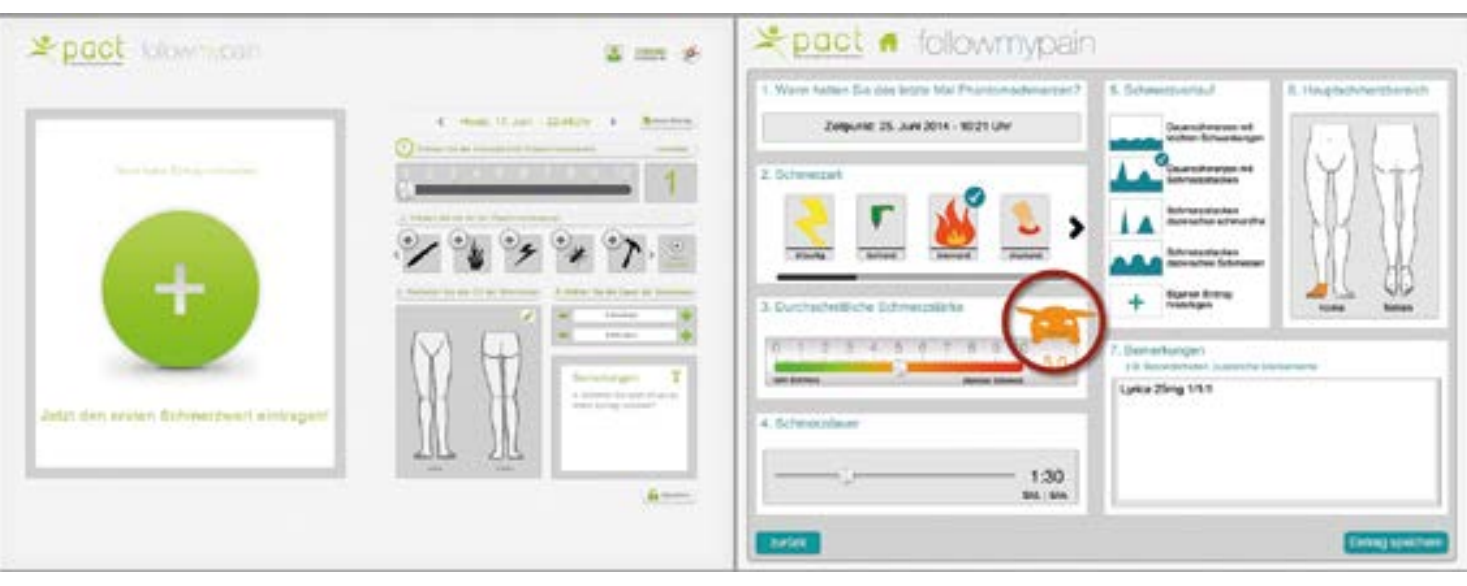

Figure 3. First graphical user interface (GU) prototype and final interface design of phantom limb pain monitoring after 7 iterative rounds

From Low to Medium-Fidelity Prototype

The coding process based on the workflow description resulted in a low-fidelity prototype of 5 different individual applications that were included in the main menu of the patient interface of the telerehabilitation platform (Figure 4): monitoring phantom limb pain, traditional mirror therapy, mobile mirror therapy facilitated by augmented reality using the tablet-integrated camera (Figure 5; Multimedia Appendix 1), mental practice including relaxation exercises and limb laterality recognition training.

The main menu was also coded as 1 individual application and featured additional functions such as an overview of exercise programs and training history, background information, personal settings, or communication with a personal therapist and other patients (eg, short message system, videoconferencing).

The main menu of the therapist interface of the low-fidelity prototype integrated 4 different applications in a coherent overview, to enable easy access for the professional: personal and medical data of patients, monitoring of phantom limb pain and self-administered exercises, creation of individual exercise programs, and communication with individual patients (Figure 4). In addition, the main menu contained

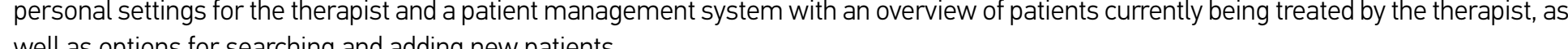




(1)

Figure 4. Low-fidelity prototype of patient and therapist interfaces of the telerehabilitation platform.

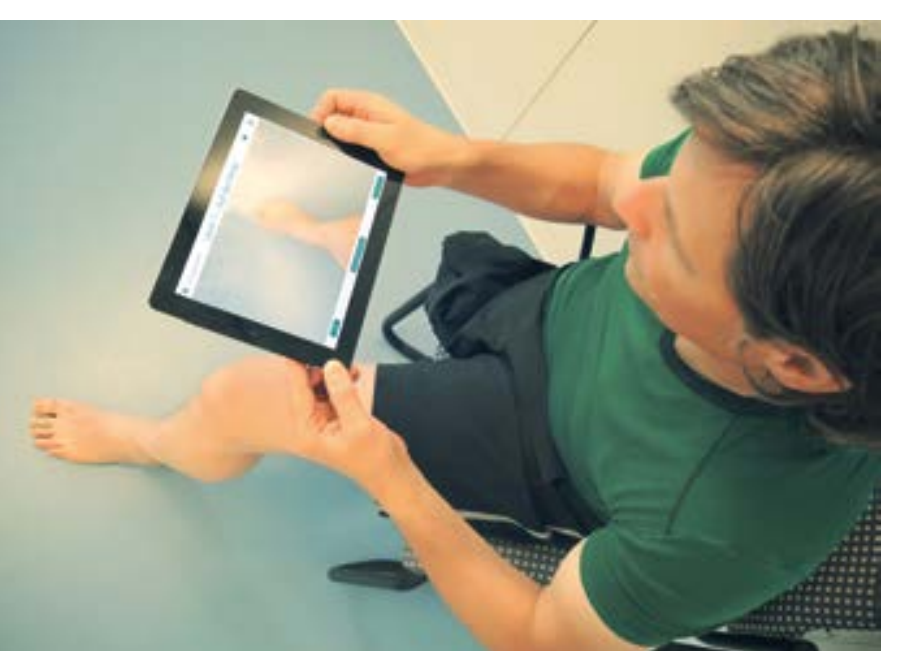

Figure 5 . Mobile mirror therapy facilitated by augmented reality using the
tablet-integrteg
Heuristic Evaluation

The group of evaluators who rated the usability according to Nielsen criteria identified several usability problems in the low-fidelity prototype, as shown in Table 3. Usability problems were found to occur in different areas of the prototype (eg, log-in, profile settings, exercise programs). All usability problems the did not provide sufficient information about the system status during various tasks such as sending messages. medium-fidelity prototype of the telerehabilitation plator

\begin{tabular}{|c|c|c|c|c|c|}
\hline Tipe of heuristax & 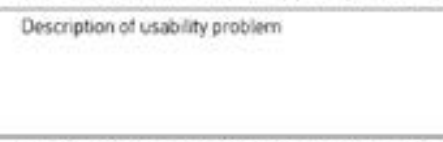 & 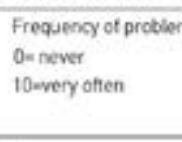 & 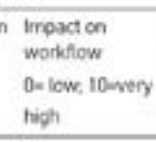 & 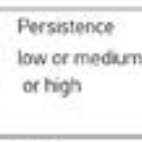 & 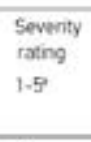 \\
\hline 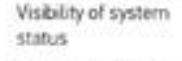 & 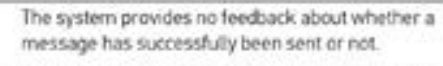 & & & High & \\
\hline 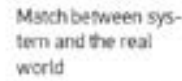 & 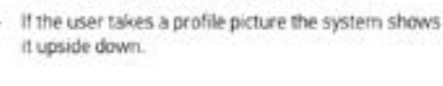 & & 3 & Medum & 3 \\
\hline 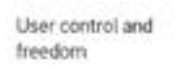 & 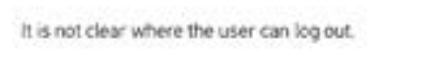 & 10 & ? & Low & “ \\
\hline 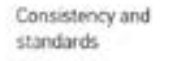 & 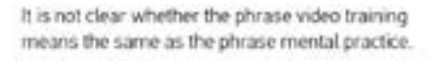 & 2 & 0 & Low" & 2 \\
\hline Erece prevention & 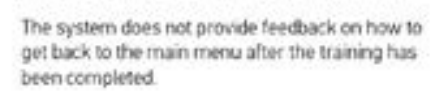 & 10 & 8 & Medum & 4 \\
\hline 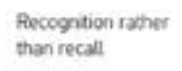 & 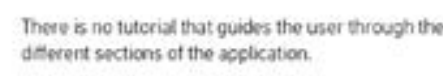 & 2 & ${ }^{3}$ & High & 3.4 \\
\hline 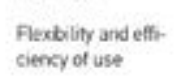 & 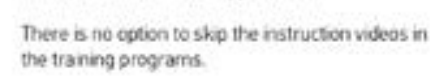 & 10 & 5 & Medum & 4 \\
\hline 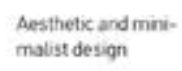 & 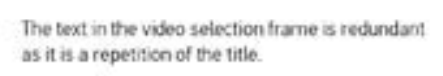 & 8 & 0 & Low & ${ }^{2}$ \\
\hline 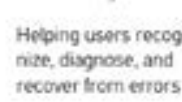 & 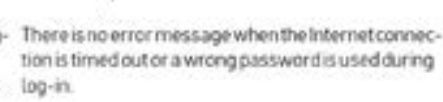 & & 10 & Modum & “ \\
\hline 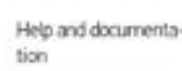 & 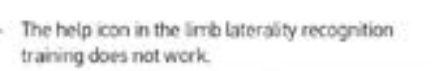 & ${ }^{2}$ & 1 & High & 2 \\
\hline
\end{tabular}


Phase 3: Field Testing in Routine Care, Redesign and Development of High-Fidelity Prototype

During the 4 weeks of field testing of the medium-fidelity prototype in routine care, patients and therapists reported additional usability

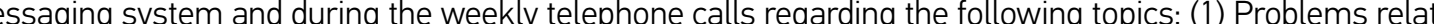
to the Internet connection (eg, delayed data transfer and log-in): (2) Messaging system (eg. message is not completely visible in the text fields, no confirmation it the message was successfully sent, message not received by user): (3) Data management (eg. system displays wrong dates and patient scores); (4) Patient management (eg, failure to add new patients and save a tailored exercise program): and (5) Interface design (eg, overlap of text and icons, missing icons).

The software development team continuously redesigned the medium-fidelity prototype. As soon as a new version of the telerehabilitation prototype was available, the soffware for patients and therapists was updated so they were able to test it in routine care.

High-Fidelity Prototype

After all major bugs had been fixed, additional graphics such as a home button were added to the patient interface. In addition, some elements to facilitate patient compliance The button to select a training program was replaced by a button "immediate action" to enable patients to immediatly start mobile mintor therapy in case of an acute attack of phantom limb pain. Tapping on the colored circles starts the individual exercise programs A new tutorial delete patients was included in the therapist interface (Figure 6).

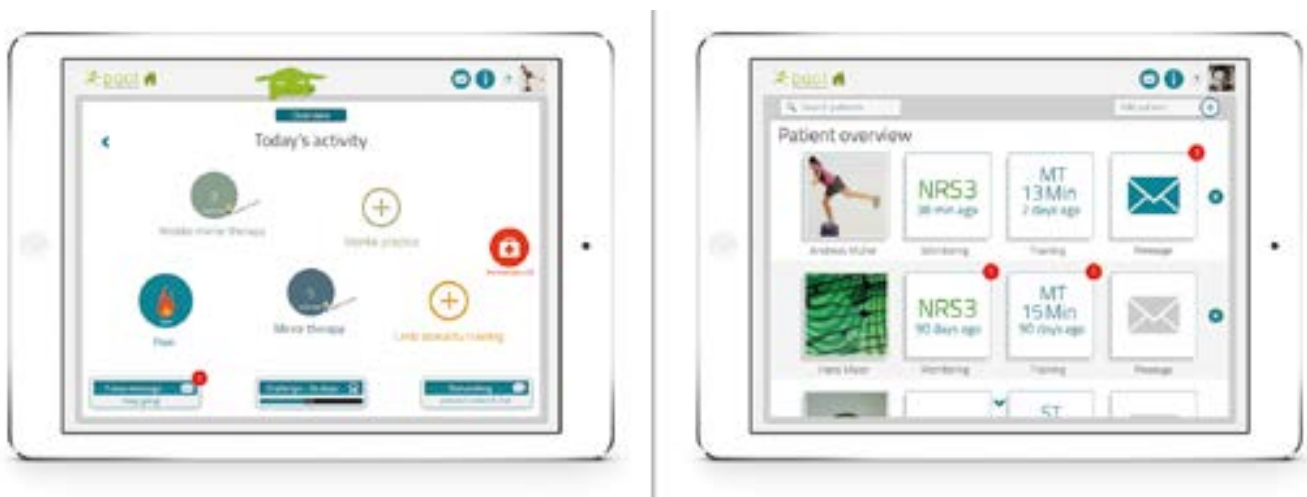

Figure 6. High-fidelity prototype of patient and the-
rapist interfaces of the telerehabilitation platorm.
DISCUSSION

In this project, an interdisciplinary soffware development team consisting of several stakeholders (patients, health care professionals, researchers, and information technology IIT experts) took part in designing and developing a mobile telerehabilitation platform for patients with phantom limb pain by means of an iterative user-centered design process. Each of the 3 research questions was answered in a separate phase of the process.

Principal Findings

The first phase of the study aimed to identify the requirements defined by patients and therapists regarding the content and functions of a telerehabilitation platform and how these requirements could be prioritized to develop a first prototype of the platform.

The users defined an extensive list of requirements ( $N=127$ ) regarding the topics of monitoring, training programs, communication, settings, background information, log-in, general requirements, and patient management The limited time and budget available meant that not all requirements could be incorporated in the platform. Hence, it was essential to have a decision aid based on clear criteria that enabled the first prototype of the telerehabilitation platform. To this end we developed a decision matrix reflecting the views of various stakeholders based on 3 different criteria best avalable evidence 9 importance of the requirement, and the technical complexity (time or money) of implementing the requirement in the platform.

The first 2 criteria were clear and straightforward to use The last criterion, however required frequent discussion with the software team and turned out to be an important and restricting factor in deciding whe such as "monitoring the phantom limb pain" were technologically easy to deve lop and implement whereas some others, such as "perceived position and range of motion of phantom limb" were technologically complex to design. It has to be mentioned that depending on the user characteristics (eg. age, experience in using IT) it was difficult for some users to provide reasonable information regarding the content and functionalities of the platform. For this reason, some requirements were only mentioned by 1 or 2 users nonetheless providing valuable information In order to also meet the needs that were mentioned by minority of users, 3 members of the researh team that rat the priority of requirements decided hether these requienents provided important information that should be taken into account Overall the decision matrix was very helpful and enabled us to systematically rate and prioritize all requirements.

The second phase of the study was used to assess how the user interface of the telerehabilitation platform could be designed to match the 
critical user requirements and how the interface could best be translated into a medium-fidelity prototype.

It appeared to be crucial to involve the users and other stakeholders early and often in the design process, that is in line with results from a recent scoping review .0 $^{40}$ The potential future users were shown mock-ups and prototypes of graphical user interfaces of the low and medium-fidelity prototypes of the platform, incorporating the predefined user requirements. During this iterative process, the users were able to check whether their requirements had been sufficiently addressed. They highly appreciated the possibility to co-create the application with the interdisciplinary software team. In particular, participants were enthusiastic about discussing with other users their ideas regarding the functions and interface design, and to see how their feedback was incorporated in the subsequent prototypes. In addition, some functions and interface design issues that were suggested by the software team, such as adding a Facebook sign-in button, were rejected because the users did not consider them relevant. As soon as the final interface design emerged, it was important to provide the software developers with a structured and logical workflow description so that they were able to code a first prototype matching the critical user requirements. However, continuous redesign of the first prototype was required to achieve a medium-fidel ty prototype, as several usability problems were identified through heuristic evaluation. This close cooperation with the users and other stakeholders gave us valuable insights into critical requirements and resulted in a telerehabilitation platform that will most likely fit the main requirements and wishes of the end users.

Phase 3 of the project assessed the usability of the medium-fidelity prototype of the telerehabilitation platform in routine care as judged by patients with phantom limb pain and their treating therapists. This information was necessary to redesign the platform into a high-fidelity prototype. An important step during the iterative design process was field testing the platform in routine care, which contributed greatly to improving the usability of the platform. During this process, the users continuously identifed additional problems that had not been detected before through heuristic evaluation. When feld testing started, the users rated the usability of the medium-fidelity prototype as poor because of several problems such as dela What dransfer or problems regarding the login process It was important to discuss the usability problems continuously with the sofware development team and to regulary provide the users with an improved version of the platform to gradually increase its usability to achieve a high-fidelity prototype. However, at a certain point in the development process we had to stop improving the platform and start the multicenter trial in order to evaluate the effects of the plafform 10 This time was diffeut to set as there are no forma criteria to decide when to stop the prototype design process. Development of the platform stopped after all critical issues had been resolved and time ad budaet testrictions did not allow any more reported bugs to be addressed despite the fact that tess critical malfunctions kep occurring. The latter imples that in the platform that is currently being evaluated in a multicenter trial $1^{10}$ there could still be some minor malfunctions which can potentially influence user acceptance.

Strengths and Limitations

In our experience, it is important to take sufficient time for the different stakeholders to get to know and understand each other. It is necessary that the different stakeholders learn to speak each other's language in order to work effectively together and correctly transform the wishes and requirements of the users into the design of the tool. Even though the involvement of the users and other stakeholders made the process time-consuming, we believe that it is a crucial factor in building an eventually successful and user-friendly platform.

A potential limitation of this study could be that the same sample of patients and therapists (except for the patients who were recruited for usability testing in routine care) was used throughout the development process of the telerehabilitation platform. This enabled patients and therapists to check whether the requirements, which they defined, were sufficiently addressed in the first prototypes of the platform. However using the same sample also carries the risk that the views of novel users without prior knowledge regarding the platform are insufficiently addressed. This may have resulted in a lower number of reported usability problems. This potential underestimation of usability problems was tackled by including novel patients who were not familiar with the technology during field-testing in routine care Patients and therapists who participated in field testing had limited time to practice in using the telerehabilitation platform. However this time frame seemed appropriate to evalute the usability and ease of use of the system as it reflected the situation of a first-time user ${ }^{4 i}$ Field multicenter trial, 10 in which patients use the telerehabilitation platform over a period of 6 months.

\section{Comparison With Prior Work}

Prioritization of user requirements is still a challenge in software engineering. ${ }^{42}$ Recently, it has been recommended that requirements should be prioritized from a user point of view.22 There are many difficulties in defining which factors should be taken into account when setting the priorities. For example. Moisiadic43 argues that prioritizing requirements should involve representatives from different stakeholders with a vested interest in the success of the development project To our knowledge ours is one of he fir incorperting the views of different stakholders to systematically rate and prioritize user requirements within a teleheath project.

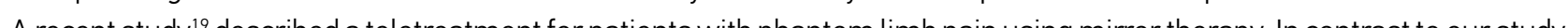
consisted solely of email instructions by a physicin on how to deliver self-administered mimor her 
have many other requirements regarding the functionalities of a telerehabilitation platform, such as monitoring the phantom limb pain, communication with a personal therapist and other patients, as well as tailored management of the training programs.

In recent years, several telerehabilitation plattorms have been developed for different patient groups, such as those with musculoskeletal neurological 45 or pulmonary conditions. 46 However, it remains unclear whether these platforms were developed following a strict usercentered approach. Lack of user acceptance is one of the major barriers to the deployment of services in many telehealth projects 4.4 .48 mainly because relevant user preferences and usability issues have not been taken into account.4. Early and frequent involvement of end users the design process, as presented in this study, could prevent some of the problems described previously. We followed the human-centered design principlest ${ }^{49}$ with the goal of designing a system that is modeled in accordance with the characteristics, tasks, and requirements of the end users. However in software engineering herea and evaluation method might therefore have led to different results.

\section{Recommendations for Future Research}

Given the limited research efforts being invested to systematically involve the end users in the design of new teletreatments, the findings of this study (eg the use of a decision matrix) could be applied in future telehealth projects. Sharing the experiences with tools for human-centere design processes will eventually lead to a better understanding of ways to develop user-friendly teletreatments, will enable comparison with products and the efficacy of different methods, and will ultimately lead to higher degrees of user acceptance for eHealth solutions. Mirror therapy has shown promising results in reducing phantom limb pain in 3 controlled studies, however the evidence is still limited 7.8 It is stil through mirror therapy could be more suitable for alternative methods such as virtual or augmented reality 50 Compared with the mirror ing thereby making the visul illusion more vivid and real, which has been shown to be correlated with the effects of the treatment ${ }^{6}$ The results of our multicenter trial10 will yield information about the potential effects of mirror therapy and the telerehabilitation platform in treating phantom limb pin in routie care, and will indicate further points for improvement of the platform. Within this trial we will also assess user acceptance of the service using a questionnaire based on the technology acceptance model. .3.2. $^{2}$
Conclusions

This study involved developing a mobile telerehabilitation platform for patients with phantom limb pain through an iterative user-centered design process. Our findings underline the importance of involving the users and other stakeholders in an iterative design process by our project, as well as the need for clear criteria to identify critical user requirements. The decision matrix presented here incorporates the views of various stakeholders and might help others systematically rate and prioritize user requirements. The reported findings and lessons learned might be of interest to health care providers, researchers, software designers, and other stakeholders when designing and evaluating new teletreatments. They may also potentially increase the likelihood of user acceptance of these applications.

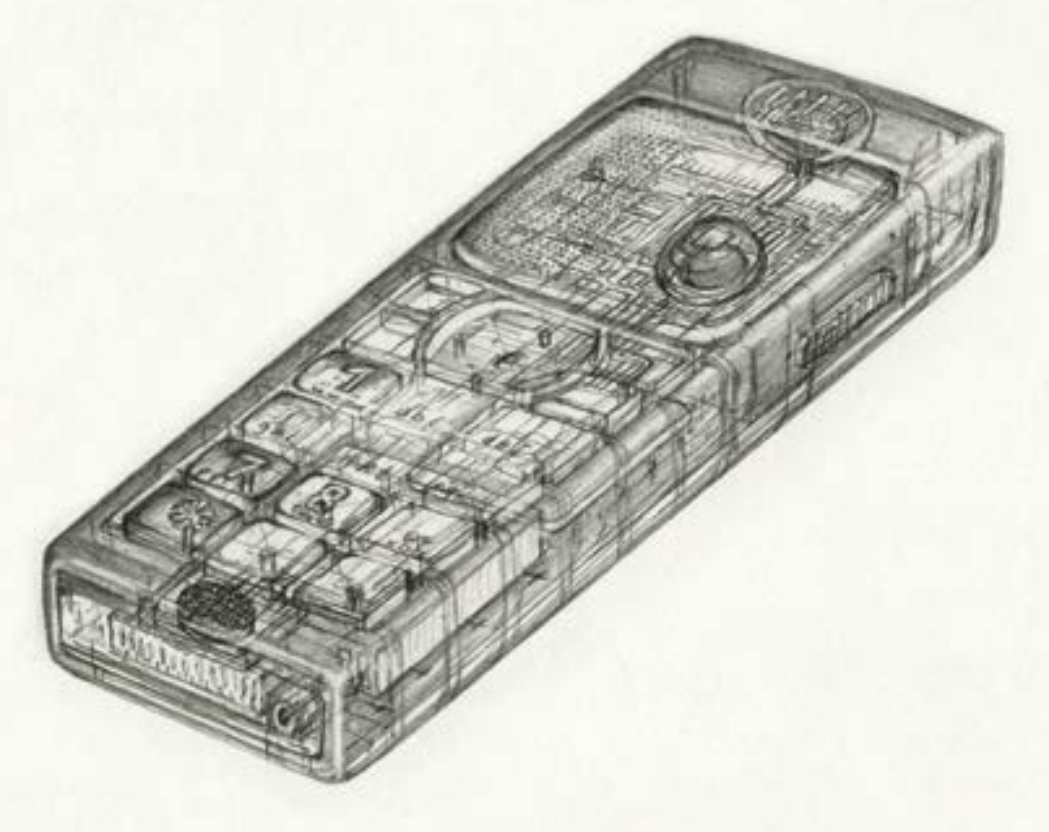




\section{REFERENCES}

Ephraim PL, Wegener ST, Mackenzie EJ, Dillingham TR and Pezzin LE. Phantom pain, residual limb pain, and back pain in amputees: results of a national survey. Arch Phys Med Rehabil. 2005; 86: 1910-9. Hanley MA, Ehde DM, Jensen M, Czerniecki J, Smith DG and Robinson LR. Chronic pain associated with upper-limb loss. Am J Phys Med Rehabil. 2009; 88: 742-51; quiz 52, 79.

3. Bekrater-Bodmann R, Schredl M, Diers M, et al. Post-amputation pain is associated with the recall of an impaired body representation in dreams-results from a nation-wide survey on limb amputees. PloS one. 2015; 10: e0119552.

Vaso A, Adahan HM, Gjika A, et al. Peripheral nervous system origin of phantom limb pain. Pain. 2014; 155: 1384-91.

5. Flor $\mathrm{H}$, Nikolajsen $\mathrm{L}$ and Staehelin Jensen T. Phantom limb pain: a case of maladaptive CNS plasticity? Nat Rev Neurosci. 2006; 7: 873-81.

Foell J, Bekrater-Bodmann R, Diers M and Flor H. Mirror therapy for phantom limb pain: brain changes and the role of body representation. Eur $J$ Pain. 2014; 18: 729-39,

. Rothgangel AS, Braun SM, Beurskens AJ, Seitz RJ and Wade DT. The clinical aspects of mirror therapy in rehabilitation: a systematic review of the literature. Int J Rehabil Res. 2011; 34: 1-13.

Barbin J, Seetha V, Casillas JM, Paysant J and Perennou D. The effects of mirror therapy on pain and motor control of phantom limb in amputees: A systematic review. Ann Phys Rehabil Med. 2016; 59: 270-5, Rothgangel A, Braun S, de Witte L, Beurskens A and Smeets R. Development of a Clinical Framework for Mirror Therapy in Patients with Phantom Limb Pain: An Evidence-based Practice Approach. Pain Pract. 2016; 16: 422-34.

10. Rothgangel AS, Braun S, Schulz RJ, et al. The PACT trial: PAtient Centered Telerehabilitation: effectiveness of limb amputation: protocol of a multicentre randomised controlled trial. J Physiother. 2015; 61: 42; discussion
11. Darnall BD and Li H. Home-based self-delivered mirror therapy for phantom pain: a pilot study. J Rehabil Med. 2012; 44: 254-60.

12. Kairy D, Tousignant M, Leclerc N, Cote AM, Levasseur M and Researchers $T$. The patient's perspective of in-home telerehabilitation physiotherapy services following total knee arthroplasty. Int J Environ Res Public Health. 2013; 10: 3998-4011.

13. Finkelstein J, Lapshin O, Castro H, Cha E and Provance PG. Home-based physical telerehabilitation in patients with multiple sclerosis: a pilot study. J Rehabil Res Dev. 2008; 45: 1361-73.

4. Agostini M, Moja L, Banzi R, et al. Telerehabilitation and recovery of motor function: a systematic review and meta-analysis. J Telemed Telecare. 2015; 21: 202-13.

15. Brennan DM, Mawson S and Brownsell S. Telerehabilitation: enabling the remote delivery of healthcare, rehabilitation, and self management. Stud Health Technol Inform. 2009; 145: 231-48.

16. Hailey D, Roine R, Ohinmaa A and Dennett L. Evidence of benefit from telerehabilitation in routine care: a systematic review. J Telemed Telecare. 2011; 17: 281-7.

17. Huis in 't Veld RM, Kosterink SM, Barbe T, Lindegard A, Marecek T and Vollenbroek-Hutten MM. Relation between patient satisfaction, compliance and the clinical benefit of a teletreatment application for chronic pain. J Telemed Telecare. 2010; 16: 322-8.

18. Kairy D, Lehoux P. Vincent C and Visintin M. A systematic review of clinical outcomes, clinical process, Ssociated with telerehabilitation. Disabil Rehabil. 2009; 31: 427-47.

19. Gover-Chamlou A and Tsao JW. Telepain Management of Phantom Limb Pain Using Mirror Therapy. Telemed J E Health. 2016; 22: 176-9.

20. Holden RJ and Karsh BT. The technology acceptance model: its past and its future in health care. Journal of biomedical informatics. 2010; 43: 159-72.

21. Shah SG, Robinson I and AlShawi S. Developing medical device technologies from users' perspectives a theoretical framework for involving users in the development process. Int J Technol Assess Health Care. 2009; 25: 514-21. 
22. Finch TL, Mair FS, O'Donnell C, Murray E and May CR. From theory to ,measurement' in complex interventions: methodological lessons from the development of an e-health normalisation instrument. BMC Med Res Methodol. 2012: 12:69.

23. Davis FD. Perceived Usefulness, Perceived Ease of Use, and User Acceptance of Information Technology. MIS Quarterly. 1989; 13: 319-40.

24. Davis FD, Bagozzi RP and Warshaw PR. User Acceptance of Computer Technology: A Comparison of Two Theoretical Models. Management Science 1989: 35: 982-1003.

25. Venkatesh, Morris, Davis and Davis. User Acceptance of Information Technology: Toward a Unifed View. MIS Quarterly. 2003; 27: 425.

26. Venkatesh V. Creation of Favorable User Perceptions: Exploring the Role of Intrinsic Motivation. MIS Quarterly. 1999; 23: 239.

27. van Gemert-Pijnen JE, Nijland $\mathrm{N}$, van Limburg M, et al. A holistic framework to improve the uptake and impact of eHealth technologies. J Med Internet Res. 2011; 13: el11.

28. Dingsøyr T, Nerur S, Balijepally $V$ and Moe N. A decade of agile methodologies: Towards explaining agile software development. J Sys Soft. 2012; 85: 1231-21.

29. Hsieh HF and Shannon SE. Three approaches to qualitative content analysis. Qual Health Res. 2005; 15: 1277-88. 30. Clegg D and Barker R. Case Method Fast-Track: A RAD Approach. Reading: Addison-Wesley; 2004 Clegg D and B WA.1990, p. 249-56.

2. Nielsen J. Heuristic evaluation. In: Nielsen J, and Mack, R.L., (ed). Usability Inspection Methods. New York NY. John Wiley \& Sons, 1994

33. SchmalzL L, Ragno $\mathrm{C}$ and Ehrsson $\mathrm{HH}$. An alternative to traditional mirror therapy: illusory touch can reduce phantom pain when illusory movement does not. Clin J Pain. 2013; 29: e10-8.

34. Mercier $\mathrm{C}$ and Sirigu A. Training with virtual visual feedback to alleviate phantom limb pain. Neurorehabil Neural Repair. 2009; 23: 587-94
Moseley GL. Graded motor imagery for pathologic pain: a randomized controlled trial. Neurology. 2006; 67: 2129-34

36. Sumitani M. Miyauchi S. McCabe CS, et al. Mirror visual feedback alleviates deafferentation pain, depending on qualitative aspects of the pain: a preliminary report Rheumatology. 2008; 47: 1038-43.

37. Beaumont $\mathrm{G}$, Mercier C, Michon PE, Malouin F and Jackson PL. Decreasing phantom limb pain through observation of action and imagery: a case series. Pain Med. 2011; 12: 289-99.

38. Maclver K, Lloyd DM, Kelly S, Roberts $N$ and Nurmikko T. Phantom limb pain, cortical reorganization and the therapeutic effect of mental imagery. Brain. 2008; 131: 2181-91.

Giraux P and Sirigu A. Illusory movements of the paralyzed limb restore motor cortex activity. Neuroimage. 2003; 20 Suppl 1: S107-11

40. Matthew-Maich N, Harris L, Ploeg J, et al. Designing, Implementing, and Evaluating Mobile Health echnologies for Managing Chronic Conditions in Older Adults: A Scoping Review. JMIR Mhealth Uhealth. 2016; 4: e29.

4. Johnson $\mathrm{CM}$, Johnson TR and Zhang J. A user-centered framework for redesigning health care interfaces. J Biomed Inform. 2005; 38: 75-87.

2. 2 Lehtola L Kappinen Mand Kujal S Requirements Prioritization Challenges in Practice Proceedings of the 5th International Conference on Product Focused Software Process Improvement. Kansai Science City, Japan 2004, p. 497- 508.

43. Moisiadis F. The fundmentas of prititing requirements Proceedings of Systems Engineering Test \& Evaluation Conference. Sydney, Australia 2002.

44. Cottrell MA, Galea OA, O'Leary SP, Hill AJ and Russell TG. Real-time telerehabilitation for the treatment of musculoskeletal conditions is effective and comparable to standard practice: a systematic review and meta-analysis. Clin Rehabil. 2017; 31: 625-38.

45. Khan F, Amatya B, Kesselring J and Galea M. Telerehabilitation for persons with multiple sclerosis, Cochrane Database Syst Rev. 2015: CDO10508. 
46. Lundell S, Holmner A, Rehn B, Nyberg A and Wadell K. Telehealthcare in COPD: a systematic review and meta-analysis on physical outcomes and dyspnea. Respir Med. 2015; 109: 11-26.

Broens $\mathrm{TH}$, Huis in't Veld RM, Vollenbroek-Hutten MM, Hermens $\mathrm{HJ}$, van Halteren AT and Nieuwenhuis 2007; 13: 303-9.

48. Huis in 't Veld RM, Widya IA, Bults RG, Sandsjo L, Hermens HJ and Vollenbroek-Hutten MM. A scenario

guideline for designing new teletreatments: a multidisciplinary approach. J Telemed Telecare. 2010; 16: 302-7.

Maguire M. Methods to support human-centred design. Int J Human-Computer Studies. 2001; 55: 587-634.

Ortiz-Catalan M, Guethmundsdottir RA, Kristoffersen MB, et al. Phantom motor execution facilitated by

machine learning and augmented reality as treatment for phantom limb pain: a single group, clinical trial in patients with chronic intractable phantom limb pain. Lancet. 2016; 388: 2885-94. 


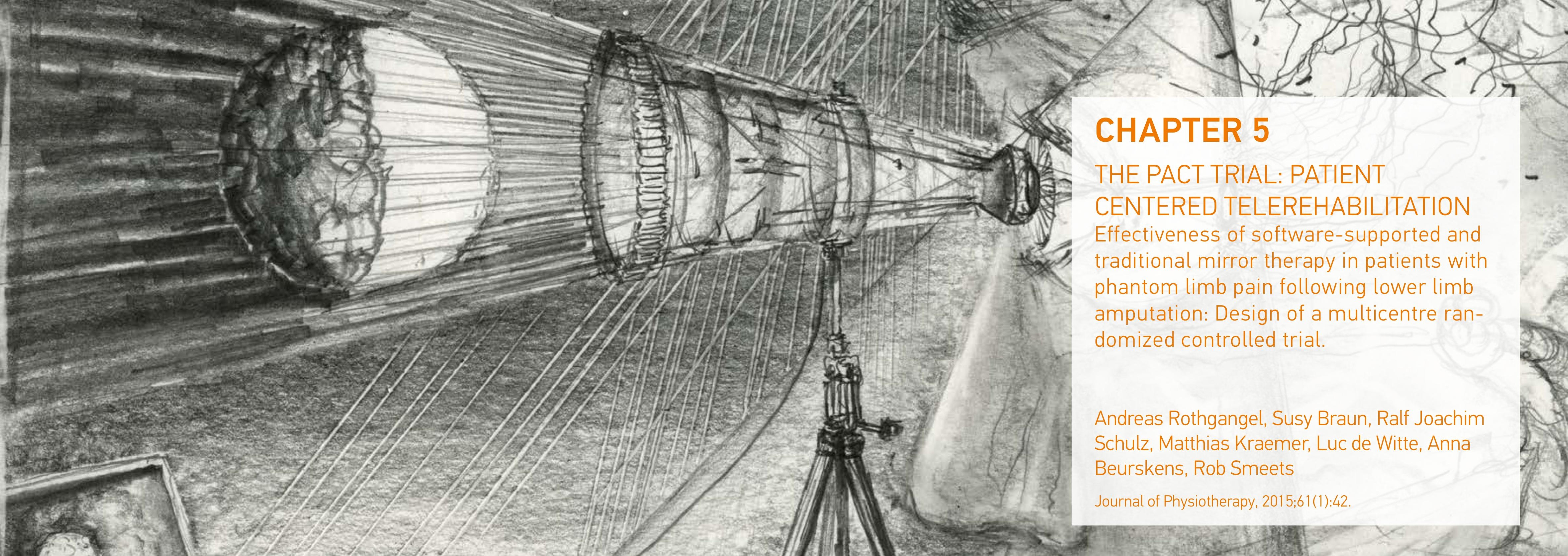




\section{ABSTRACT}

INTRODUCTION

Non-pharmacological interventions such as mirror therapy are gaining increased recognition in the treatment of phantom limb pain (PLP).

However, the evidence in patients with PLP is still weak. In addition, compliance to self-delivered exercises is generally low. The aim of this

However, the evidence in patients with PLP is stil weak. In adartion,
randomized controlled study is to investigate the effectiveness of mirror therapy supported by telerehabilitation on the intensity, duration and randomized controlled study is to investigate the effectiveness of mirror therapy supported by telerehabilitation on the intensity, duration and
frequency of phantom limb pain and limitations in daily activities compared to traditional mirror therapy and care as usual in patients following lower limb amputation.

\section{METHODS}

A three-arm multi-centre randomized controlled trial will be performed. Patients will be randomly assigned to care as usual, traditional mirror

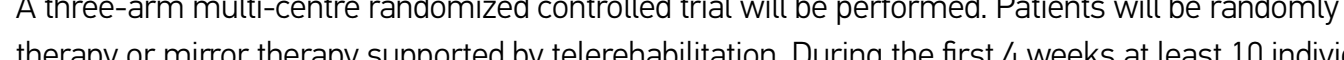
therapy or mirror therapy supported by telerehabilitation. During the first 4 weeks at least 10 individual sessions will take place in every group. After the frs 4 weeks patients are encouraged to perform self-delivered exercises over a periodo 6 weeks. Oncomes will be assessed at 4 and 10 weeks atter basello and a 6 months follow-up. Primary outcome measures include he average intensity or phantom limb pain during the peek. Secondary outcome measures include the different perceived effect pain specific selfefficacy and quality of life.

\section{DISCUSSION}

Several questions concerning the study design that emerged during the preparation of this trial are discussed. It is described how these questions were addressed and arguments for the choices made are given 


\section{INTRODUCTION}

Significant differences exist in the incidence of lower limb amputations worldwide, ranging from 46.1 to 9.600 per 100.000 in the diabetic population and 5.8-31 per 100.000 in the total population.' The existence of phantom limb pain (PLP) is a major complaint of patients following amputation. Up to $90 \%$ of patients after amputation suffer from chronic PLP, ${ }^{2 \cdot 6}$ leading to limitations in daily activities and reduced quality of life. ${ }^{2}$-10 ${ }^{-10}$ As many patients with amputation live at home, there is need for efficient self-management strategies to handle phantom limb pain sustainably. These

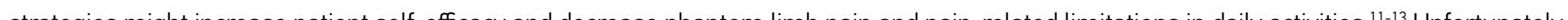

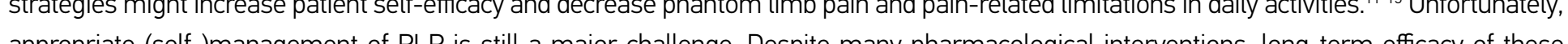
作 aldition int additional recent systematic review we showed that to date, only two small randomized controlled trials (RC) demonstrate that mirror herapy is effective in reducing phantom of treatment protocols showing positive results, one could advise that mirror therapy should be conducled with a minimum frequency of one session per day over a period of several weeks. However, this treatment frequency is often beyond the resources available in clinical practice. In addition, long-term adherence to self-delivered exercises is generally low.2 It has been suggested that additional support can be useful to discuss problems that occur during self-management, to individually modify the treatment program and to increase long-term adherence to treatment." The latter can be achieved by using telerehabiltation, which enables remote support of patient's autonomy and monitoring of selfmanagement.2-25 An important element in the development and implementation of telerehabilitation systems is a thorough analysis of user requirements to prevent lack of user acceptance. $2.0-50$ Until now, user involvement and participation is offen neglected when such applications are designed. $2 ., 29$ To date, no telerehabilitation exists, that is tailored to the needs of patients with phantom limb pain and the preferences of physical and occupational therapists who are treating those patients with mirror therapy.

This article describes the study protocol of the randomized controlled study of the PAtient Centred Telerehabilitation (PACT) project (fig. 1).

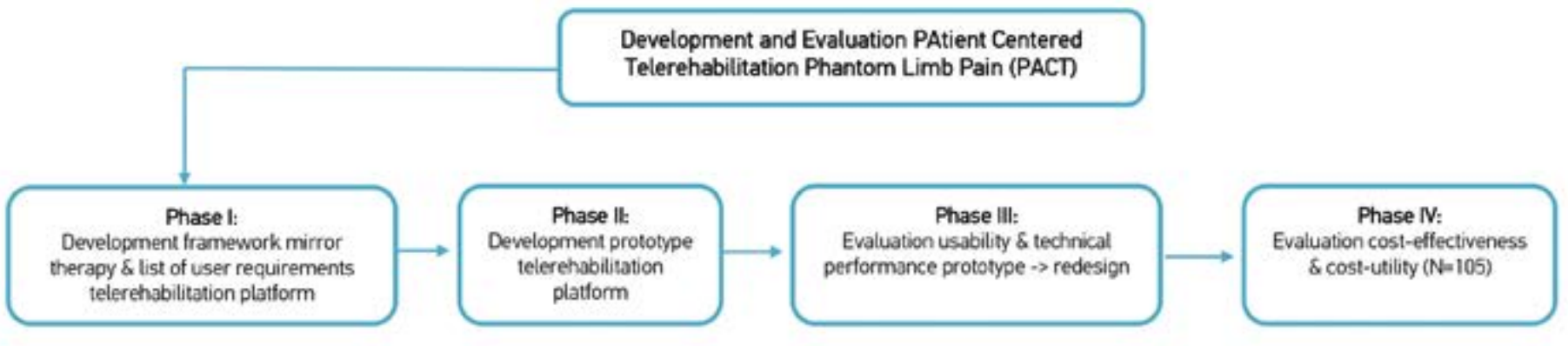

Figure 1. Overview of the different phases within the PACT project

\section{Objectives}

The overall aim of the three-arm randomized controlled study is to investigate the effectiveness of mirror therapy supported by telerehabilitation on the intensity, duration and frequency of phantom limb pain and daily activities compared to traditional mirror therapy and usual care without mirror therapy in patients following lower limb amputation.

In the PACT project, we applied a user-centered approach to develop a telerehabilitation for patients with phantom limb pain following lower limb amputation. Figure 1 shows an overview of the different phases within the PACT project. An extensive description of this developing process and results will be described in another publication. 
Research questions

For further information see also figure 2 and table 1 .

1) Are there any differences in treatment effect between a 4-weeks intervention using care as usual (group A) and 4-weeks traditional mirror therapy (group $B \& C$ ) on intensity duration and frequency of phantom limb pain and pain related limitations in daily activities in patients with therapy (group B\&C) on intensity, duration and requen,

2) Are there any differences in treatment effect between traditional mirror therapy followed by mirror therapy supported by telerehabilitation (group C) compared to traditional mirror therapy followed by self-delivered mirror therapy (group B) and care as usual without traditiona mirror therapy (group A) on intensity, duration and frequency of phantom limb pain, pain related limitations in daily activities, pain specific self-efficacy and quality of life?

3) What is the cost-effectiveness and cost-utility of traditional mirror therapy followed by mirror therapy supported by telerehabilitation (group C) compared to traditional mirror therapy followed by self-delivered mirror therapy (group B) and care as usual without traditional mirror therapy (group A) from a societal perspective?

\section{METHOD}

Design

A three-arm multi-centre randomized controlled trial will be performed involving patients following lower limb amputation from multiple centres (rehabilitation clinics and private practices). Patients will be randomly assigned to one of the three following conditions. A: 4-weeks sensomotor exercises to the intact limb without a mirror (care as usual) followed by 6 weeks self-delivered care as usual; B: 4-weeks traditional mirror therapy followed by 6-weeks self-delivered mirror therapy without support (experimental condition 1) or C: 4-weeks traditional mirror therapy followed by 6-weeks self-delivered mirror therapy supported by telerehabilitation (experimental condition 2). All baseline measurements (TO) will be obtained after recruitment of participants and before random assignment to either the care as usual or experimental groups (see fig. 2). Endpoints of the trial will be assessed directly after the first four weeks intervention phase (T1), after six weeks of self-management (T2), and at six months follow-up (T3).

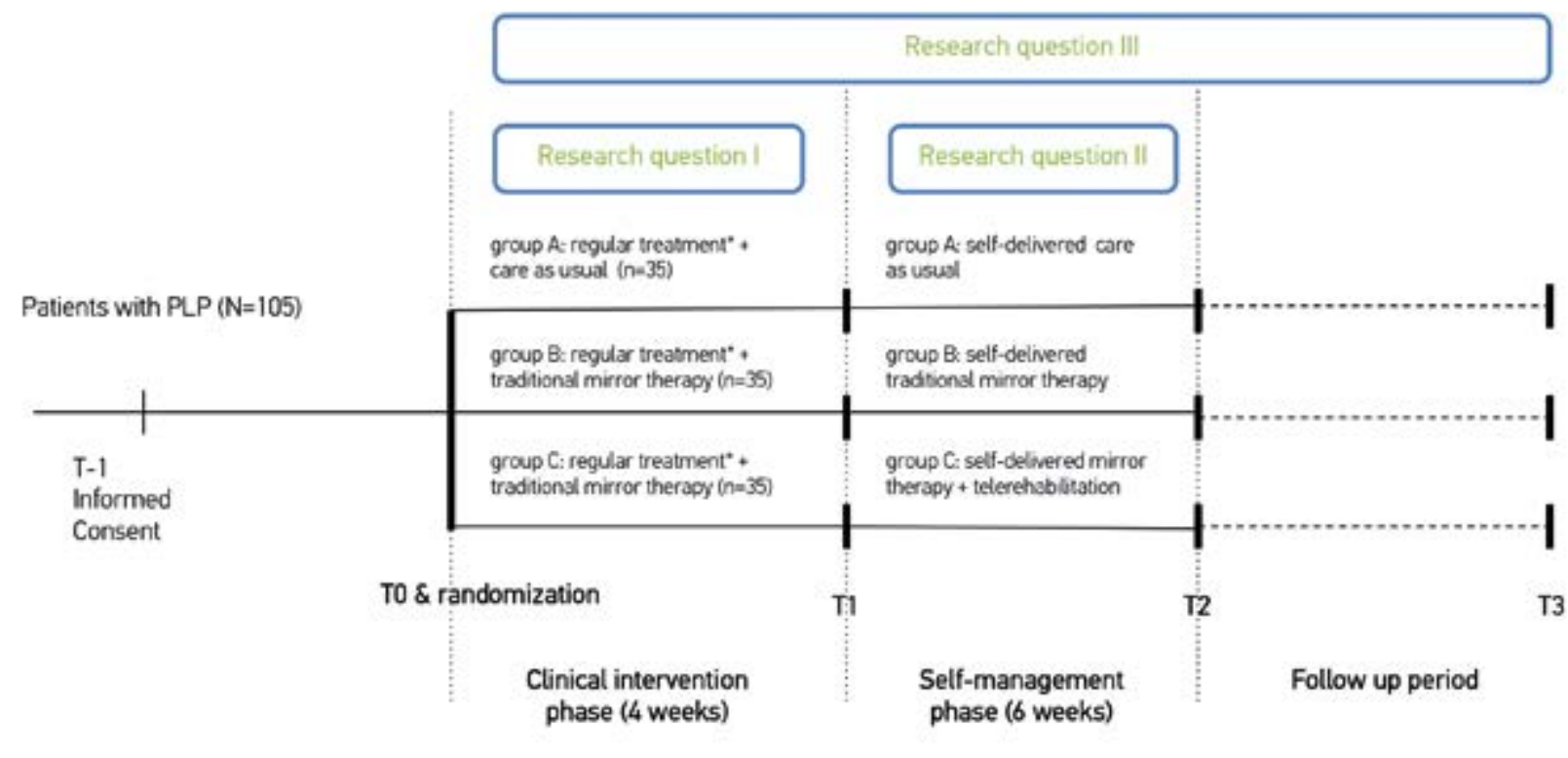

Legend: T-1 11-4 days before T0; TO=baseline; $T 1=4$ weeks following T0; T2=10 weeks following T0; T3= 6 months following T0; PLP= phantom limb pain; Figure 2. Overview on the study design of the randomized controlled study of the PACT projed 
Participants

This trial will commence recruitment in May 2014 and is expected to be completed in July 2015. Patients after lower limb amputation will be recruited through treating physicians at participating centers or allied health professionals. In addition, confederative centers, patient support groups and online advertisement assist in recruiting eligible participants living at home. To be engaged in this trial, patients have to fulfill the following selection criteria

a) Lower limb amputation

At least since one week constant or intermittent phantom limb pain (PLP) with an average intensity of at least score 3 on the 11-point numeric rating scale (NRS) and a minimum frequency of one episode of PLP per week

c) Sufficient cognitive, communicative and motor functions to be able to use the telerehabilitation service, to concentrate for at least 15 minutes on the mirror image and to follow instructions and questionnaires; this is based on clinical judgment of recruiting physicians or therapists.

Exclusion criteria:

d) Not able to follow at least 10 individual sessions during the first 4 weeks.

Bilateral amputation, severe co-morbidity (e.g. stroke) or pain affecting the intact limb; this prevents

engagement in the prescribed exercise programs of the study.
Severe psychiatric disorder that precludes the patient from participating in the trial.

) Intensive course of mirror heraph in

\section{Sample size calculation}

The calculation of sample size is based on the primary endpoint, the mean intensity of the last episode of PLP, measured on an 11-point
NRS. A mean difference of 2 (sd=2.25) points on the NRS between condition A (control group) and B (traditional mirror therapy) is regarded as a clinically relevant difference $31-33$ While assuming an intra-class correlation of $10 \%$, for a power of $80 \%$ and a significance level of $0.05,30$ patients are required per condition ${ }^{34}$ However, we expect a dropout rate of approximately $20 \%$ so we aim to include 35 patients per condition, 105 in total.

Randomization

Participants will be individually randomized per center using a computerized, blocked randomization scheme, with block sizes of six, to achieve an equal distribution of participants across all groups after every sixth patient in each centre. No further stratification will take place. ${ }^{35} \mathrm{An}$ independent blinded research assistant outside the participating centers will administer the randomization sequence. For every center, the randomization scheme and corresponding group allocation will be stored on the personal mobile phone of the research assistant secured by password. Only the administering person and its deputy will have access to the file. After recruitment and baseline measurement, each patient will be registered and the principal investigator (AR) will be informed by phone. The latter will contact the administering person to disclose group allocation and will communicate the assigned treatment to the treating therapist. This randomization procedure will be identical for all participating centers.

Interventions

Participating physicians and therapists will be trained before the beginning of the trial regarding the following topics: (1) selection criteria and process of patient recruitment: (2) aims, design and measurements of the study: (3) content of the interventions. For all interventions, a standardized treatment protocol has been developed

In the rehablititich all interventions will be given additionally to the regular treatments 'add-on') The regular treatment is defined as

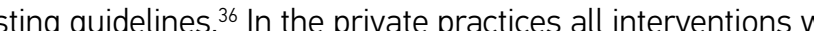
any other regular treatment.

vention phase of four weeks (TO-T1), treatment frequency will account for at least ten individual sessions lasting 30 minutes for every condition. Beside the face-to-face sessions, all patients will be encouraged to conduct exercises on their own as much as they want. Appropriate exercise material and a diary to record treatment frequency will be handed out to every patient. The same therapist 
will treat patients in the two experimental groups. Another therapist, who does not treat patients from the experimental groups, will treat patients in the control group. During the self-management phase of six weeks (T1-T2) until the follow-up measurement six months after To (T3) patients will perform self-delivered exercises as much as they want Table 1 gives an overview of the content of all interventions used within the RCT.

Table 1. Content of interventions used in this study

\begin{tabular}{|c|c|c|c|}
\hline \multirow[t]{2}{*}{ Group } & \multicolumn{3}{|c|}{ Content intervention } \\
\hline & $\begin{array}{l}\text { Clinical intervention phase } \\
(\mathrm{TO}-\mathrm{T} 1)\end{array}$ & $\begin{array}{l}\text { Self-management } \\
\text { phase (T1-T2) }\end{array}$ & $\begin{array}{l}\text { Follow-up period } \\
(12-T 3)\end{array}$ \\
\hline $\begin{array}{l}\text { Control intervention } \\
\text { (group A) }\end{array}$ & Regular treatment" + care as usual & Self-delivered care as us & al without support \\
\hline $\begin{array}{l}\text { Experimental } \\
\text { intervention I }\end{array}$ & $\begin{array}{l}\text { Regular treatment }{ }^{*}+\text { traditional } \\
\text { mirror therapy }\end{array}$ & $\begin{array}{l}\text { Self-delivered traditiona } \\
\text { support }\end{array}$ & mirror therapy without \\
\hline $\begin{array}{l}\text { Experimental } \\
\text { intervention II } \\
\text { (group C) }\end{array}$ & $\begin{array}{l}\text { Regular treatment" }+ \text { traditional } \\
\text { mirror therapy. } \\
\text { Introduction to telerehabilitation } \\
\text { during the last week }\end{array}$ & $\begin{array}{l}\text { Self-delivered mirror } \\
\text { therapy supported by } \\
\text { telerehabilitation }\end{array}$ & $\begin{array}{l}\text { Self-delivered mirror } \\
\text { therapy supported by } \\
\text { telerehabilitation } \\
\text { without contact to } \\
\text { therapist }\end{array}$ \\
\hline
\end{tabular}

Control intervention (group A)

Patients in the control group will conduct the same sensomotor exercises with the intact limb using the same treatment dose as patients in the traditional mirror therapy group (group B), but without using a mirror (=care as usual). During all exercises patients will observe the movements of the intact limb. At the end of the clinical intervention phase, patients will be encouraged to continue exercises on their own until the follow-up measurement (T3).
Experimental intervention I (group B) categories:

Observation of various positions in the mirror (creation of the 'mirror illusion')

b) basic motor exercises (e.g. flexion-extension movements)

c) sensory stimulation exercises (e.g. using different brushes)

e) mental practice of phantom exercises using the mirror (e.g. alternately observing movements in the mirror and mentally practicing these movements with the phantom)

In the first sessions, the therapist will determine for every patient which exercises are most effective in achieving a vivid sensomotor sensation in the phantom limb. The latter seems to be an important factor regarding the effects of a mirror therapy intervention. $3{ }^{37.38}$ Subsequently, these exercises will be trained during the remaining sessions. At the end of the clinical intervention phase (T1), patients will be encouraged to continue mirror therapy on their own until the follow-up measurement (T3).

\section{Experimental intervention II (group C)}

The second experimental intervention consists of traditional mirror therapy followed by self-delivered mirror therapy supported by addition patients will be trained on how to use the telerehabilition at the end of the clinical intervention phase before dischen $B$. patient will be loaned a tablet- $\mathrm{PC}$ and a set of training materials for the duration of the self-manament phase. The telereh bilitation uses perent components

Background information on phantom limb pain and given interventions

b) Monitoring of phantom limb pain (e.g. intensity \& frequency of pain) 
Self-delivered exercises to treat phantom limb pain (videos on mirror therapy and mental practice, augmented reality using the tablet-integrated camera, limb laterality recognition training, relaxation

d) Communication with therapist and other patients suffering from phantom limb pain

At the end of the clinical intervention phase patients will be instructed to use the telerehabilitation as often as they want in the daily situation During the six-weeks self-management phase (T1-T2) patients can communicate with the treating therapist in case of problems arising with the exercises. During the follow-up period (T2-T3) patients will be allowed to use the telerehabilitation but without support of the treating therapist.

Outcome measures \& procedure

The recruting therapist whl assess all outcomes at baseline (TO). All measurements at the end of the intervention and follow-up phases (T) T3) will be performed by an independent, blinded research assistant. The research assistant will mail all questionnaires to the patients and assist patients by phone in completing the questionnaires. The assistant will ask patients not to reveal their assigned treatment during the measurement Table 2 gives an overview of al measurements oblained duing his sudy. As is com Ill not be possible to blind patients or therapists for treatment condition. ${ }^{3}$
Table 2. Overview of outcomes, measurement instruments and -moments used in the PACT study Data Time point Aim of measurement

Patient charocteristics

Comparison of baseline characteristics

Progenostic variables

TO

Prediction of treatment effect

$\begin{array}{ll}\text { CEQ Expectancy regarding treatment effect } & \text { TO } \\ \text { Treatment frequency, prosthesis usage, position } & \text { TO, T1, T2, T3 } \\ \text { of phantom limb, etc. } & \end{array}$

Primayour

(1)

Frequens. Itensity of PLP

To, T1. T2, T3

Limitations on 'body functions/structures' level

TO. T1, T2. T3

PSFS \&. PDI: Pain related limitations in daily

TO, T1, T2, T3

TO, T1, T2, T3

Limitations on 'body functions/structures'

Q $0-50-51$.

To, T2, T3

GPE: Overall treatment effect

T1. T2.

Limitations on 'activities' leve

GPE: Overall treatment effect

$T 0, T 1, T 2, T 3 \quad$ Analysis of environmental factor

Limitations on 'activities' and 'participation' level

During intervention period

og: Treatment frequency, medication intake

Acceptance questionn

Daily

$\begin{array}{ll}\text { Dally } & \text { Monitoring of treatment } \\ \text { T1. T2 T3 } & \text { Monitoring of direct/indirect costs }\end{array}$

Co-Interventions, integrity check

T2 2 T

Assessment of acceptance of telerehabilitation
Process evaluation

Legend $T-1=1-4$ days before $T 0 ; T 0=$ baseline; $T 1=4$ weeks following $T 0 ; T 2=10$ weeks following $T 0 ; T 3=6$ months following $T 0$;

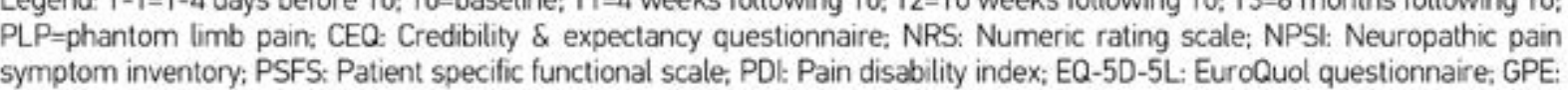

symptom inventory; PSFS: Patient specific functional scale, PDI: Pain disability index; EQ-5D-5L: EuroQuol
Global perceived effect scale. FESS: Fragebogen zur Erfassung der schmerzpezifischen Selbstwirksamkeit. 
Patient characteristics

At baseline (To) the following patient characteristics will be assessed: age, gender, educational level, profession and family status. In addition, the following variables regarding the amputation will be recorded: side, level, date and reason for amputation, type and frequency of usage of value ${ }^{38}$ After randomisation and treatment allocation, patients' treatment expectancy and rationale credibility will be measured with the credibility and expectancy questionnaire (CEQ), ${ }^{0}$ as these factors might represent non-specific reed regarding their prognostic value and to compare characteristics of the three groups at baseline.

\section{Primary outcome measures}

The mean intensity of phantom limb pain during the last week will be assessed using an 11-point numeric rating scale (NRS) ( $0=$ no pain, $10=$ pain at its worst). Additionally, the frequency and duration of pain episodes will be scored. The NRS shows good validity and reliability. The minimal clinically relevant difference on the NRS on group level is 2 points.

\section{Secondary outcome measures}

The different dimensions of PLP will be assessed through the German Version of the Neuropathic Pain Symptom Inventory (NPSI-G).2. The NPS includes 10 descriptors and two temporal items to discriminate and quantify clinically relevant dimensions of neuropathic pain. Each of the 10 descriptors uses an 11-point NRS (0=no pain, 10=pain at its worst) to score the intensity of the pain description. The NPSI shows good construct validity, high test-retest reliability, is sensitive to change and has been translated into several languages. 43.44

Pain related limitations in daily activities will be assessed by the German version of the patient specific functional scale (PSFS) (Heldmann in press). Three, for each individual patient important activities that are hampered due to phantom limb pain will be scored using an 11-point numeric rating scale (NRS) (0=no limitations, $10=$ not possible to perform activity).5.47 Sufficient validity, reliability and sensitivity to change have been established in patients with different pain syndromes with a minimal clinically relevant difference of 2 points or $30 \%$ on group level. $5.47-4.49$
Additionally, limitations in daily activities will be assessed by a more generic measure, the German version of the Pain Disability Index (PDI).50 The degree of limitations in daily activities will be scored on seven topics using an 11-point numeric rating scale (NRS) ( $0=n o$ limitations, 10=not possible to perform activity). The seven topics from the PDI will be complemented by two items, sleep and mood, from the brief pain

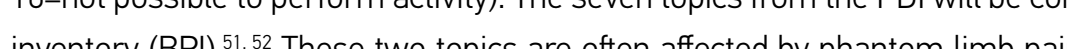

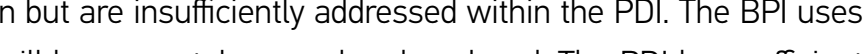
psychometric properties with a minimal clinically relevant difference of 9 points. ${ }^{53.5}$

Quality of Life will be measured on five domains using the German version of the EuroQol Questionnaire (EQ-5D-5L). ${ }^{5.59}$ Each item is scored using a five-point scale (1=no problems, 2=slight problems, $3=$ =moderate problems, $4=$ =evere problems, $5=$ =nable to do/extreme problems). Additionally, the EQ-5D-5L uses a visual analogue scale (VAS) to score overall health ( $0=$ worst imaginable health; $100=$ best imaginable health). Psychometric properties of the EQ-5D-5L are sufficient, except a ceiling effect. ${ }^{59}$

Global perceived effect (GPE) of treatment will be rated on a 7-point scale (-3=extreme worsening; +3=extreme improvement) to assess patients' subjective perceptions of recovery. Test-retest reliability of the GPE scale is excellent. However, GPE ratings seem to be strongly influenced by current status. ${ }^{60}$

Pain specific self-efficacy will be assessed using the German version of the pain self-efficacy questionnaire (PSEQ).61 The questionnaire (a) confident). ${ }^{62}$ The PSEQ has good internal consistency and test-retest reliability.

\section{Additional variables}

Several additional variables will be assessed to analyse feasibility, integrity and compliance of the treatment.

Feasibility \& Integrity

At the end of the self-management phase (T2) patients' and therapists' satisfaction and acceptance of the telerehabilitation service will be 
evaluated with 9 items on an 11-point scale ( $0=$ strongly disagree, $10=$ absolutely agree). The items on acceptance have been derived from the Technology Acceptance Model (TAM) ${ }^{63,64}$ including questions on intention to use, perceived usefulness, ease of use and application-specific self-efficacy. In order to evaluate integrity of the treatment, all therapists will be encouraged to record any deviation from the treatment protocol, adverse effects or other particularities after every session in a log.

Additional information on co-interventions or medical problems will be registered in a log as well and a standardized drop out evaluation takes place over the study period (TO-T3). An independent research assistant will register reasons for drop out and possible adverse effects if patients give their consent. As our statistical analysis is based on intention to treat, we will ask patients to give consent on registering data after withdrawal.

Compliance

In the telerehabilitation group, software will assess exercise frequency and duration through data logging. In the mirror therapy and control group a log will be used to assess frequency of (self-delivered) exercises. The treating therapist will regularly check these data.

\section{Economic Evaluation}

Costs and effects will be evaluated from a societal perspective. In order to assess direct and indirect costs, a cost questionnaire will be used in every group at all measurement moments following baseline (T1-T3). Direct costs include health care utilization in general (e.g. visits to health care providers, drug of consulations will be multiplied by the cost of each visit to calculate thal drect costs. Idrect costs include data from loss of productivity (e.g. illness related absence from paid and unpaid work). Patients will be encouraged to register only resources that are used in relation to phantom limb pain. Costs for development and implementation of the telerehabilitation service will also be calculated

\section{Data analysis}

Demographic data of patients as well as primary and secondary outcomes will be analysed at baseline (TO) on significant differences between the groups. In case of significant differences between groups analysis of covariance will be performed. For all measurement moments following baseline (T1-T3) mean differences between groups and effect sizes (Cohens' d) will be calculated for the outcome variables. In addition, a repeated measures design withe used with primary and secondary outcomes as dependentvariables, group as between-subjects factor and moment of measurement as the within-subjects factor. Prognostic variables will be identified through regression analysis and data from the logs will be analysed qualitatively. A subgroup analysis will be performed on the variables age and gender. Statistical analysis of group differences will be performed according to the intention to treat principle.

In the economic evaluation differences in costs and effects between all groups will be compared using the incremental cost-effectiveness ratio including the net costs per reliable and clinically relevant improved case of pain. The costs and effectiveness of the interventions will be displayed by a cost effectiveness plane. In addition, an incremental cost-utility ratio will be calculated incorporating the net costs per quality adjusted life years (QALY) gained

Ethical considerations

Before study inclusion, each participant

will be sufficiently informed about the

study purposes and content by providing

study purmoses and coviding

sufficient time (at least 2 work

sufink abou (ady past 2 working days)

think about study participation and to sign

informed consent. Table 3 gives an overview

of the ethical considerations. The study has

been approved by the Ethics committee of

the Medical Faculty of Cologne University,

Cologne, Germany (approval no. 13-304).

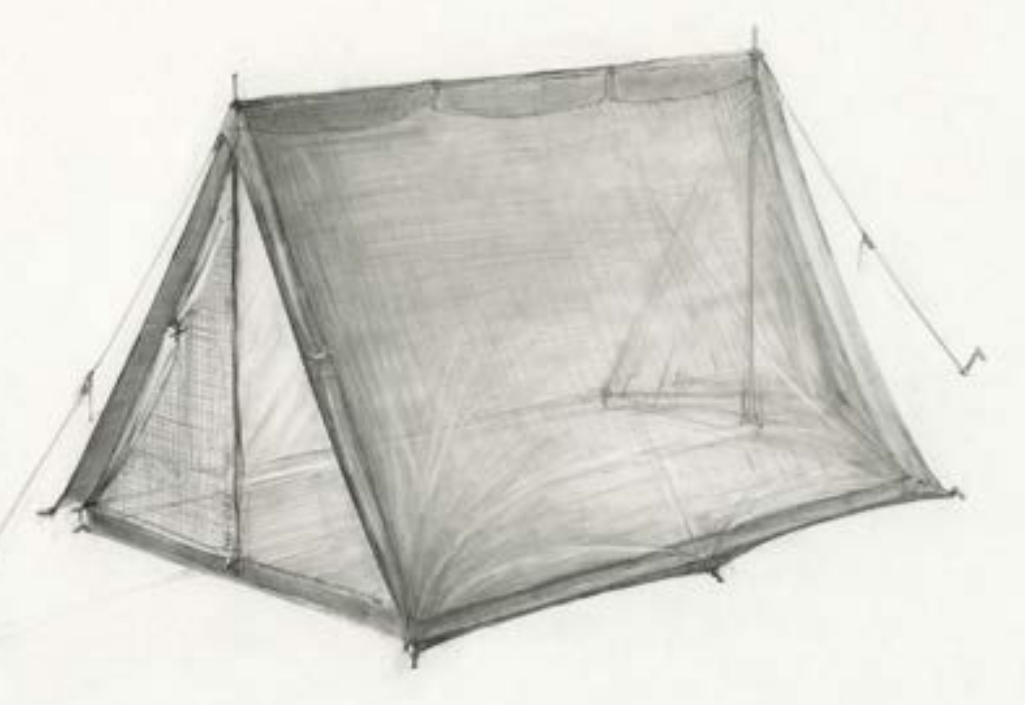


Table 3. Overview of ethical considerations

\begin{tabular}{|c|c|}
\hline Ethical aspect & Comment \\
\hline $\begin{array}{l}\text { Are participants sufficiently informed } \\
\text { about the study? }\end{array}$ & $\begin{array}{l}\text { Before study inclusion, each participant is sufficientlty informed about the study } \\
\text { purposes and content by providing an informed consent form. Patients have sufficient } \\
\text { time (at least } 2 \text { days) to think about study participatition and sign informed consent. } \\
\text { Patients are free to withdraw from study participation without giving any reason and } \\
\text { any consequences on their medical treatment }\end{array}$ \\
\hline $\begin{array}{l}\text { What are the additional risks for } \\
\text { participants? }\end{array}$ & $\begin{array}{l}\text { The existing literature suggests, that during mirror therapy adverse effects (e.g. } \\
\text { dizziness, nausea) might occur over a short period of time. }\end{array}$ \\
\hline $\begin{array}{l}\text { What are the potential benefits for } \\
\text { participants? }\end{array}$ & $\begin{array}{l}\text { The treatment can have a positive effect on phantom limb pain, disability and quality } \\
\text { of life. Patients are informed about the background of phantom limb pain and are } \\
\text { trained in self-management strategies. Participants can get in contact with other } \\
\text { patients suffering from phantom limb pain. }\end{array}$ \\
\hline $\begin{array}{l}\text { What are potential benefits from a } \\
\text { societal perspective? }\end{array}$ & $\begin{array}{l}\text { The treatment might induce positive socioeconomic effects. Several publications are } \\
\text { submitted and researchers and health care providers are trained regarding research } \\
\text { methodology and interventions. }\end{array}$ \\
\hline $\begin{array}{l}\text { What is the extra burden for } \\
\text { participants? }\end{array}$ & $\begin{array}{l}\text { Moderate extra time load through additional measurements not incorporated in usual } \\
\text { care and self-delivered exercises. }\end{array}$ \\
\hline
\end{tabular}

DISCUSSION

The overall aim of this randomized controlled study is to investigate the effectiveness of mirror therapy supported by telerehabilitation on the intensity, duration and frequency of phantom limb pain and daily activities compared to traditional mirror therapy and care as usual in patients following lower limb amputation.

The available literature shows good auality of evidence that mirror therapy is effective as an additional intervention in improving recovery of

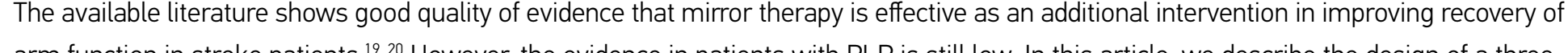
arm function in stroke patients. Dowever, he vidence in palients With PLP is still low. In this antic, we describe the design of a threearm multi-centre ran the effects of mirror therapy on phantom limb pain and the second question will determine the additional effects of the telerehabilitation. The latter is an important question as a sufficient frequency of face-to-face visits is not possible given the fact that resources in clinical practice are scarce. In addition, long-term adherence to self-delivered exercises is generally low. ${ }^{21}$ In the near future, this discrepancy between therapy demand and avallable resources will even increase due to demographic changes. Growing financial pressures in the health care system and the increase in chronic diseases will shift rehabiltation more and more towards self-management of patients.11-13,65 Telerehabilitation could help to solve at least some of these problems.

During the preparation of the PACT-project several questions concerning the study design needed to be addressed. II the following section, we describe how we dealt with these questions and argue the choices made.

\section{User-centred design}

Putting the users at the centre during the development of an e-health application is essential to prevent lack of user acceptance..$^{29}$ The latter is often neglected when such applications are designed resulting in barriers to deployment 28.29,66 In the developmental phase of the PACT a wer-centred design, performing semi-structured interviews to elicit user requirements concerning the content of the telerehabilitation (publication in preparation). This process resulted in a multitude of data making it impossible to integrate all individual requirements into the design of the telerehabilitation. Accordingly, we developed a criterion checklist to structure and prioritize functions which should be integrated within the telerehabilitation and which should not. This checklist contains criteria on 'the available evidence from the literature', whether 'the majority of users mentioned the item', whether there was 'agreement between patients' and therapists' wishes' and 'how technically complex it would be to build the designated function'. Based on these four criteria we graded the priority of 
the individual requirements enabling us to choose the most important functions that were consequently integrated into the design of the telerehabilitation. After the first prototype was established we tested its usability through an iterative process in which user feedback was continuously incorporated into the design of the revised prototype. In our view, this user-centred design was very helpful to facilitate user acceptance of the telerehabilitation.

Justification of the intervention

In our systematic review on the clinical aspects of mirror therapy ${ }^{20}$ we showed that there is still no consensus on treatment and patient characteristics when designing a mirror therapy treatment. In order to standardize the intervention we developed a clinical protocol for mirror therapy in stroke patients. ${ }^{67}$ Development of the protocol was guided by an evidence-based approach in which we merged the best available evidence, clinical experiences of a group of physical and occupational therapists and the preferences and experiences of stroke patients. Using the same approach, we have developed a similar protocol for mirror therapy in patients with phantom limb pain (in preparation). This protocol contains the following exercise categories that were also incorporated into the telerehabilitation: creation of a vivid mirror illusion, basic motor exercises, sensory training, functional motor exercises and mirror-facilitated mental practice. In addition, based on analysis of user requirements, we developed 'mobile' interventions that can be used by patients outside their homes without a mirror such

In our view, the treatment frequency of at least ten individat sessions in addition to self-delivered exercises during the four weeks clinical intervention phase should be sufficient to achieve a clinically revent reduction derived from clinical experience and the fact that daily sessions would not be practical for patients living at home. Nevertheless, patients and therapists are encouraged to maximize treatment intensity as far as possible.

The control intervention consists of senso-motor exercises to the intact limb without a mirror (care as usual). This was chosen to ensure sufficient contrast between groups but on the other hand to provide an intervention that also could have at least some effect on phantom limb pain. Results from other studies suggest that treatments to the contralateral limb might also alleviate phantom pain. ${ }^{7.78}$ However, we believe that the effects of mirror therapy are superior to the control intervention.
Justification of selection criteria

Little is known about which patient characteristics are important when choosing eligible patients for mirror therapy. ${ }^{20}$ Therefore, we kept selection criteria as pragmatic as possible. Given the fact that a clinically relevant change in pain on the NRS is 2 points $5^{3-33}$ patients must have a minimum average intensity of phantom limb pain of score 3 on the NRS to be able to detect significant differences between groups. We will exclude patients who followed an intensive course of mirror therapy in the recent past that is defined as more than six individual sessions during the last three months. This cut-off was chosen because in the German health care system mirror therapy as part of physical therapy is during the last three months. This cut-off was chosen because in the German health care system mirror therapy as part of physical therapy is
often prescribed once with an amount of six sessions. In our view, to achieve sustainable effects through mirror therapy, at least ten sessions are required. I If a patient followed a more intensive course of mirror therapy before this time frame of three months, we think that possible

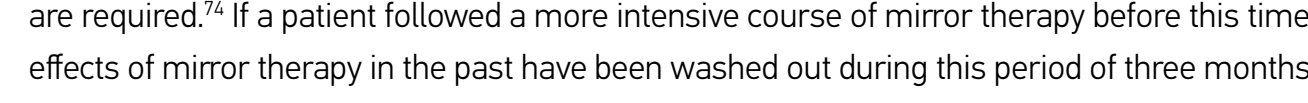

Justification of outcome measures

We tried to follow the recommendations from the Initiative on Methods, Measurements, and Pain Assessment in Clinical trials (IMMPACT) ${ }^{79}$ and the guidelines from the Neuropathic Pain Special Interest Group (NeuPSIG) ${ }^{80}$ as far as possible when choosing appropriate measurement instruments. We considered choosing additional instruments to monitor physical performance (e.g. activity monitor) $)^{81}$ but as many patients suffer from PLP in situations in which they are less active, ${ }^{2.7}$ we felt that the value of these data could not justify the additional load imposed an patients. Regarding the economic evaluation we delibered about whether we should use a cost diay or auestionnire in order to (2) questionnaire because of pragmatic reasons and reduced patient burden.

Final remark

Non-pharmacological interventions such as mirror therapy are getting increased recognition in the treatment of patients with phantom limb pain. We hope that this study will contribute to the body of evidence for mirror therapy in PLP and expand the knowledge on how to deliver mirror therapy in clinical practice and increase compliance after discharge by using information and communication technology. 


\section{REFERENCES}

Moxey PW, Gogalniceanu P, Hinchliffe RJ, et al. Lower extremity amputations--a review of global variability in incidence. Diabet Med. 2011; 28: 1144-53.

Kern U, Busch V, Muller R, Kohl M and Birklein F. Phantom limb pain in daily practice--still a lot of work to do! Pain Med. 2012; 13: 1611-26.

Ephraim PL, Wegener ST, Mackenzie EJ, Dillingham TR and Pezzin LE. Phantom pain, residual limb pain

and back pain in amputees: results of a national survey. Arch Phys Med Rehabil. 2005; 86: 1910-9.

Clark RL, Bowling FL, Jepson F and Rajbhandari S. Phantom limb pain after amputation in diabetic

patients does not differ from that after amputation in nondiabetic patients. Pain. 2013; 154: 729-32.

Kooijman CM, Dijkstra PU, Geertzen JH, Elzinga A and Van Der Schans CP. Phantom pain and phantom

sensations in upper limb amputees: an epidemiological study. Pain. 2000; 87: 33-41.

Richardson C, Glenn S, Nurmikko T and Horgan M. Incidence of phantom phenomena including phantom limb pain 6 months after major lower limb amputation in patients with peripheral vascular disease. Clin $J$ Pain. 2006; 22: 353-8.

Bosmans JC, Suurmeijer TP, Hulsink M, Van Der Schans CP, Geertzen JH and Dijkstra PU. Amputation, phantom pain and subjective well-being: a qualitative study. Int J Rehabil Res. 2007; 30: 1-8.

Hanley MA, Ehde DM, Jensen M, Czerniecki J, Smith DG and Robinson LR. Chronic pain associated with upper-limb loss. Am J Phys Med Rehabil. 2009; 88: 742-51.

Sinha R, van den Heuvel WJ and Arokiasamy P. Factors affecting quality of life in lower limb amputees. Prosthet Orthot Int. 2011; 35: 90-6.

Van Der Schans CP, Geertzen JH, Schoppen T and Dijkstra PU. Phantom pain and health-related quality of life in lower limb amputees. J Pain Symptom Manage. 2002; 24: 429-36.

Coleman K, Austin BT, Brach C and Wagner EH. Evidence on the Chronic Care Model in the new millennium. Health Aff(Millwood). 2009; 28: 75-85.
12. Wagner EH. Chronic disease management: what will it take to improve care for chronic illness? Ef Clin Pract. 1998; 1: 2-4.

3. Wagner EH, Bennett SM, Austin BT, Greene SM, Schaefer JK and Vonkorff M. Finding common ground: patient-centeredness and evidence-based chronic illness care. J Altern Complement Med. 2005; 11 Suppl patient-cen.

14. Alviar MJ, Hale Tand Dungca M. Pharmacologic interventions for treating phantom limb pain. Cochrane Database Syst Rev. 2011: CD006380.

15. Chan BL, Witt R, Charrow AP, et al. Mirror therapy for phantom limb pain. N Engl J Med. 2007; 357: 2206-7.

6. Maclver K, Lloyd DM, Kelly S, Roberts $\mathrm{N}$ and Nurmikko T. Phantom limb pain, cortical reorganization and the therapeutic effect of mental imagery. Brain. 2008; 131: 2181-91.

17. Moseley GL. Graded motor imagery for pathologic pain: a randomized controlled trial. Neurology. 2006; 67:2129-34

18. Ulger O, Topuz S, Bayramlar K, Sener $G$ and Erbahceci F. Effectiveness of phantom exercises for phantom limb pain: a pilot study. J Rehabil Med. 2009; 41: 582-4.

19. Thieme H, Mehrholz J, Pohl M, Behrens J and Dohle C. Mirror therapy for improving motor function after stroke. Cochrane Database Syst Rev. 2012; 3: CD008449.

20. Rothgangel AS, Braun SM, Beurskens AJ, Seitz RJ and Wade DT. The clinical aspects of mirror therapy in rehabilitation: a systematic review of the literature. Int J Rehabil Res. 2011; 34: 1-13.

21. Darnall BD and Li H. Home-based self-delivered mirror therapy for phantom pain: a pilot study. J Rehabil Med. 2012; 44: 254-60.

22. Brennan DM, Mawson S and Brownsell S. Telerehabilitation: enabling the remote delivery of healthcare, rehabilitation, and self management. Stud Health Technol Inform. 2009; 145: 231-48.

23. Hailey D, Roine R, Ohinmaa A and Dennett $L$. Evidence of benefit from telerehabilitation in routine care: a systematic review. J Telemed Telecare. 2011; 17: 281-7. 
24. Kairy D, Lehoux P, Vincent C and Visintin M. A systematic review of clinical outcomes, clinical process, healthcare utilization and costs associated with telerehabilitation. Disabil Rehabil. 2009; 31: 427-47.

25. Rogante M, Grigioni M, Cordella D and Giacomozzi C. Ten years of telerehabilitation: A literature overview of technologies and clinical applications. NeuroRehabilitation. 2010; 27: 287-304

26. Berg M. Patient care information systems and health care work: a sociotechnical approach. Int J Med Inform. 1999; 55: 87-101.

27. Berg M, Langenberg $\mathrm{C}, \mathrm{vd} \mathrm{Bl}$ and Kwakkernaat J. Considerations for sociotechnical design: experiences with an electronic patient record in a clinical context. Int J Med Inform. 1998; 52: 243-51.

28. De Rouck S, Jacobs A and Leys M. A methodology for shifting the focus of e-health support design onto user needs: a case in the homecare field. Int J Med Inform. 2008; 77: 589-60 I.

29. Huis in 't Veld RM, Widya IA, Bults RG, Sandsjo L, Hermens HJ and Vollenbroek-Hutten MM. A scenario guideline for designing new teletreatments: a multidisciplinary approach. J Telemed Telecare. 2010; 16: $302-7$.

30. Koch S and Hagglund M. Health informatics and the delivery of care to older people. Maturitas. 2009; 63. 195-9.

31. Farrar JT, Portenoy RK, Berlin JA, Kinman JL and Strom BL. Defining the clinically important difference in pain outcome measures. Pain. 2000; 88: 287-94.

32. Farrar JT, Young JP, Jr., LaMoreaux L, Werth JL and Poole RM. Clinical importance of changes in chronic pain intensity measured on an 11-point numerical pain rating scale. Pain. 2001; 94: 149-58.

Rowbotham MC. What is a ,clinically meaningful" reduction in pain? Pain. 2001; 94: 131-2. Twisk JW. Longitudinal data analysis. A comparison between generalized estimating equations and random coefficient analysis. Eur J Epidemiol. 2004; 19: 769-76,

35. Hewitt CE and Torgerson DJ. Is restricted randomisation necessary? BMJ. 2006; 332: 1506-8.

36. Greitemann B, Brueckner L, Bork A, Koller H, Middeldorf S and Schröter J. Rehabilitation nach Majoramputation an der unteren Extremitat. AWMF-Leitlinien-Register. 2010; Nr. 033/044.
37. Schmalzl L, Ragno C and Ehrsson HH. An Alternative to Traditional Mirror Therapy: Illusory Touch Can Reduce Phantom Pain When Illusory Movement Does Not. Clin J Pain. 2013;29: e10-e18.

38. Foell J, Bekrater-Bodmann R, Diers M and Flor H. Mirror therapy for phantom limb pain: Brain changes and the role of body representation. Eur J Pain. 2014; 18: 729-39.

39. Olivo SA, Macedo LG, Gadotti IC, Fuentes J, Stanton T and Magee DJ. Scales to assess the quality of randomized controlled trials: a systematic review. Phys Ther. 2008; 88: 156-75.

40. Devilly $G J$ and Borkovec TD. Psychometric properties of the credibility/expectancy questionnaire $J$ Behav Ther Exp Psychiatry. 2000; 31: 73-86.

41. Myers SS, Phillips RS, Davis RB, et al. Patient expectations as predictors of outcome in patients with acute low back pain. J Gen Intern Med. 2008; 23: 148-53.

42. Sommer C, Richter H, Rogausch JP, Frettloh J, Lungenhausen M and Maier C. A modified score to identify and discriminate neuropathic pain: a study on the German version of the Neuropathic Pain Symptom Inventory (NPSI). BMC neurology. 2011; 11: 104

43. Bouhassira D, Attal N, Fermanian J, et al. Development and validation of the Neuropathic Pain Symptom Inventory. Pain. 2004: 108. 248-57.

44. Crawford B, Bouhassira D, Wong A and Dukes E. Conceptual adequacy of the neuropathic pain symptom inventory in six countries. Health Qual Life Outcomes. 2008; 6: 62.

45. Stratford PW, Gilron I, Westaway MD and Binkley JM. Assessing disability and change on individual patients: a report of a patient-specific measure. Physiotherapy Canada. 1995; 47: 258-63.

46. Beurskens $\mathrm{AJ}$, de Vet $\mathrm{HC}$ and Koke AJ. Responsiveness of functional status in low back pain: a comparison of different instruments. Pain. 1996; 65: 71-6.

47. Beurskens AJ, de Vet HC, Koke AJ, et al. A patient-specific approach for measuring functional status in low back pain. J Manipulative Physiol Ther. 1999; 22: 144-8.

48. Cleland JA, Fritz JM, Whitman JM and Palmer JA. The reliability and construct validity of the Neck Disability Index and patient specific functional scale in patients with cervical radiculopathy. Spine (Phila Pa 1976). 2006; 31: 598-602. 
49. Pengel LH, Refshauge KM and Maher CG. Responsiveness of pain, disability, and physical impairmen outcomes in patients with low back pain. Spine (Phila Pa 1976). 2004; 29: 879-83.

50. Dillmann U, Nilges P, Saile H and Gerbershagen HU. [Assessing disability in chronic pain patients. Schmerz. 1994; 8: 100-10.

51. Cleeland CS, Ladinsky JL, Serlin RC and Nugyen CT. Multidimensional measurement of cancer pain: comparisons of US and Vietnamese patients. J Pain Symptom Manage. 1988; 3: 23-7.

52. Radbruch L, Loick G, Kiencke P, et al Validation of the German version of the Brief Pain Inventory. J Pain Symptom Manage. 1999; 18: 180-7.

53. Chibnall JT and Tait RC. The Pain Disability Index: factor structure and normative data. Arch Phys Med Rehabil. 1994; 75: 1082-6.

54. Nagel B, Gerbershagen HU, Lindena $G$ and Pfingsten M. [Development and evaluation of the multidimensional German pain questionnaire. Schmerz. 2002; 16: 263-70.

Pollard CA. Preliminary validity study of the pain disability index. Percept Mot Skills. 1984; 59: 974

56. Soer R, Reneman MF, Vroomen PC, Stegeman P and Coppes MH. Responsiveness and Minimal Clinically Important Change of the Pain Disability Index in patients with chronic back pain. Spine (Phila Pa 1976) 2011.

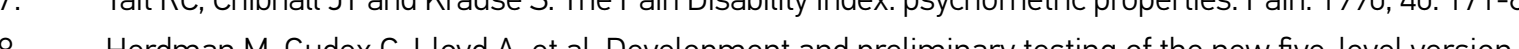
Herdma $M$ G J

59. Hinz A, Kohlmann T, Stobel-Richter Y, Zenger M and Brahler E. The quality of life questionnaire EQ-5D$5 \mathrm{~L}$ : psychometric properties and normative values for the general German population. Qual Life Res.2013

60. Kamper SJ, Ostelo RW, Knol DL, Maher CG, de Vet HC and Hancock MJ. Global Perceived Effect scales provided reliable assessments of health transition in people with musculoskeletal disorders, but ratings are strongly influenced by current status. J Clin Epidemiol. 2010; 63: 760-6 e1.

61. Mangels M, Schwarz S, Sohr G, Holme M and Rief W. Der Fragebogen zur Erfassung der schmerzspezifischen Selbstwirksamkeit (FESS). Dagnostica. 2009:55: 84-93.
N2. Nicholas MK. The pain self-efficacy questionnaire: Taking pain into account. Eur J Pain. 2007: 11: 153-63. Holden RJ and Karsh BT. The technology acceptance model: its past and its future in health care $J$ Biomed Inform. 2010; 43: 159-72.

64. Venkatesh VD, F. A theoretical extension of the technology acceptance model: Four longitudinal field studies. Management science. 2000; 46: 186-204

65. Esser PE and Goossens RH. A framework for the design of user-centred teleconsulting systems $J$ Telemed Telecare. 2009; 15: 32-9.

66. Broens TH, Huis in 't Veld RM, Vollenbroek-Hutten MM, Hermens HJ, van Halteren AT and Nieuwenhuis

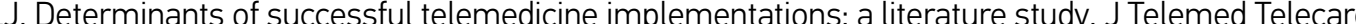
2007: 13: 303-9.

67. Rothgangel $A S$ and Braun SM. Mirror therapy: Practical protocol for stroke rehabilitation. Munich. Pflaum Verlag, 2013

68. Bowering KJ, O'Connell NE, Tabor A, et al. The effects of graded motor imagery and its components on chronic pain: a systematic review and meta-analysis. J Pain. 2013; 14: 3-13.

69. Trojan J, Diers M, Fuchs $X$, et al. An augmented reality home-training system based on the mirror training and imagery approach. Behav Res Methods. 2014 Sep;46(3):634-40.

70. Diers M and Flor H. [Phantom limb pain. Psychological treatment strategies]. Schmerz. 2013: 27: 205-11; quiz 12-3.

71. Murray CD, Pettifer S, Howard T, et al. The treatment of phantom limb pain using immersive virtual reality: three case studies. Disabil Rehabil. 2007; 29: 1465-9.

72. Cole J, Crowle $S$, Austwick $G$ and Slater DH, Exploratory findings with virtual reality for phantom limb pain; from stump motion to agency and analgesia. Disabil Rehabil. 2009; 31: 846-54.

73. Beaumont $\mathrm{G}$, Mercier C, Michon PE, Malouin F and Jackson PL. Decreasing phantom limb pain through observation of action and imarer: acase series. Pain Med 2011: 12:289-99.

74. Seidel S, Kasprian G, Furtner J, et al. Mirror therapy in lower limb amputees--a look beyond primary motor cortex reorganization. RoFo. 2011; 183: 1051-7. 
75. Bradbrook D. Acupuncture treatment of phantom limb pain and phantom limb sensation in amputees. Acupunct Med. 2004; 22: 93-7.

76. Casale R, Ceccherelli F, Labeeb AA and Biella GE. Phantom limb pain relief by contralateral myofascial injection with local anaesthetic in a placebo-controlled study: preliminary results. J Rehabil Med. 2009 41: 418-22. Davies A. Acupuncture treatment of phantom limb pain and phantom limb sensation in a primary care setting. Acupunct Med. 2013; 31: 101-4

78. Mulvey MR, Bagnall AM, Johnson MI and Marchant PR. Transcutaneous electrical nerve stimulation (TENS) for phantom pain and stump pain following amputation in adults. Cochrane Database Syst Rev. 2010: CD007264

79. Dworkin RH, Turk DC, Farrar JT, et al. Core outcome measures for chronic pain clinical trials: IMMPACT recommendations. Pain. 2005; 113: 9-19.

80. Haanpaa M, Attal N, Backonja M, et al. NeuPSIG guidelines on neuropathic pain assessment. Pain. 2011; 152: 14-27.

81. Verbunt JA, Huijnen IP and Seelen HA. Assessment of physical activity by movement registration systems in chronic pain: methodological considerations. Clin J Pain. 2012; 28: 496-504

82. van den Brink M, van den Hout WB, Stiggelbout AM, Putter $H$, van de Velde $C J$ and Kievit J. Self-reports of health-care utilization: diary or questionnaire? Int J Technol Assess Health Care. 2005; 21: 298-304 



\section{ABSTRACT}

OBJECTIVE: To compare the effects of traditional mirror therapy (MT), a patient-centred teletreatment (PACT) and sensomotor exercises without a mirror on phantom limb pain (PLP).

DESIGN: Three-arm multicentre randomized controlled

SETTING: Rehabilitation centres, hospital and private practices.

SUBJECTS: Adult patients with unilateral lower limb amputation and average PLP intensity of at least 3 on the 0-10 Numeric Rating Scale (NRS).

INTERVENTIONS: Subjects randomly received either four weeks of traditional MT followed by a teletreatment using augmented reality MT traditional MT followed by self-delivered MT or sensomotor exercises of the intact limb without a mirror followed by self-delivered exercises. MAIN MEASURES: Intensity, frequency and duration of PLP and patient-reported outcomes assessing limitations in daily life at baseline, 4 weeks, 10 weeks and 6 months.

RESULTS: In total, 75 patients received traditional MT ( $n=25)$, teletreatment $(n=26)$ or sensomotor exercises $(n=24)$. Mean (SD) age was 61 .

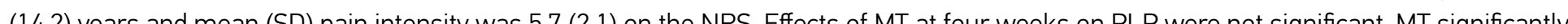
reduced the duration of PLP at six months compared to the teletreatment $(P=0.050)$ and control group $(P=0.019)$. Subroup analyses suggested signifcant effects on PLP in women, patients with telescoping and patients with a motor component in PLP. The teletreatyes suggeste signtion of PLP a six mis

CONCLUSION: Traditional MT over four weeks was not more effective than sensomotor exercises without a mirror in reducing PLP, although significant effects were suggested in some subgroups. 


\section{INTRODUCTION}

Despite the existence of many different interventions to treat patients with phantom limb pain (PLP), none has yet proven to achieve longterm effects ${ }^{1-3}$ PLP seems to be caused by maladaptive neuroplastic changes, such as the invasion of areas neighbouring the cortical representation of the amputated limb, ${ }^{4-7}$ reduced interhemispheric functional connectivity and preserved functional activity in primary sensory and motor cortices. ${ }^{8}$

Given the chronic nature of PLP' effective approaches, which address this central malplasticity, are urgently needed, since they can potentially reduce PLP sustainably. Non-pharmacological interventions such as mental practice and mirror therapy (MT) have shown promising results in reducing PLP. 10.11 However, over 20 years after Ramachandran et al. ${ }^{12}$ published the first study on MT in patients with PLP, evidence for its effectiveness is still low..$^{13.14}$ Only three controlled studies including a total of 42 amputees ${ }^{15-17}$ reported positive effects of MT during several weeks on PLP. Despite the potential merits of MT, not all patients seem to benefit from this approach.1.11,19 It seems crucial that patients routinely perform self-delivered exercises after discharge from rehabilitation to achieve long-lasting effects in the central nervous system. ${ }^{14}$ Patient-centred teletreatments (PACTs) using the principle of MT could be used to facilitate self-delivered exercises and to enhance the frequency and intensity of training, ${ }^{20}$ Within the PACT study, ${ }^{21}$ a telerehabilitation platform was developed specifically for patients with PLP, in which augmented reality MT is facilitated using the tablet-integrated camera (Supplementary Figure 1 and Video). The results of the multicentre trial within the PACT study are presented here.

The first aim of the PACT trial was to compare the immediate effects of four-week traditional MT with four weeks of sensomotor exercises without a mirror on the intensity, duration and frequency of PLP and pain-related limitations in daily activities in patients following lower limb amputation. The second aim was to assess after four weeks of traditional MT the effects of a six-week teletreatment using augmented reality MT compared to six weeks of self-delivered MT or six weeks of self-delivered sensomotor exercises without a mirror at 10 -week and 6-month follow-ups.

\section{METHODS}

The study protocol'21 of the PACT trial was approved by the Ethics committee of the Medical Faculty of Cologne University, Germany (reference no. 13-304) and registered in the Clinicaltrials.gov Register (ID NCT02076490). The principal investigator recruited nine German centres (six rehabilitation clinics, two private practices and one hospital) through existing clinical networks. The first patient registration took place in May 2014 and the last follow-up measurement was completed in September 2016. Maastricht and Zuyd University Heerlen, The Netherlands. were responsible for the conduct of the study.

Recruitment

Patients after lower limb amputation were recruited and screened for eligibility through their treating physician or allied health professional at the participating centre In addition, patients wer recruted through patient support groups and online advertisement All adult patients who had a unilateral lower limb amputation and reported an average intensity of PLP of 3 or more on the 11-point Numeric Pain Rating Scale 23 $^{23}$ and minimally one episode of PLP per week were included No restrictions were made regarding gender, age type of pain senstion or the time since amputation. In addition, eligible patients needed to have sufficient cognitive and communicative skills and motor functions in order to use the teletreatment follow instructions and understand and fill out questionnaires. The recruting healthcare professionals judged this clinically. Exclusion criter posttraumatic stress disorder), living more than $50 \mathrm{~km}$ away from a participating centre and having received more than six sessions of MT during the previous three months. All eligible participants provided written informed consent before enrolment in the study.

The principal investigator electronically generated concealed, block-randomized assignment for every centre separately with block sizes of six. He was the only person who had information to break the randomization code. No further stratifcation took place. The pacticizating centres informed the principal investigator about any new eligible patient who was registered for the study The principal investigator then provided the treating theraist with information about the assigned treatment based on a blocked random number sequence. The research assistant as well as the statisticin who alysed the data was u aware of treatment assignments. It was not possible to mask petients to treatment as they were aware of the treatment content.

Interventions After giving informed consent, patients were randomly allocated to one of the following three interventions: four weeks of traditional MT dew delvedMT (group B) 
before the start of the trial. To avoid contamination of treatments as much as possible, patients who received traditional MT during the first four weeks (groups A and B) were treated by other therapists than patients allocated to the control group (group C).

During the first four weeks, all therapists were instructed to deliver at least 10 individual sessions of the allocated intervention, each lasting 30 minutes. Before discharge at four weeks, the treating therapist instructed patients on how to perform the allocated exercises for the next six weeks themselves and provided the questionnaires that were required for follow-up measurements at 10 weeks and 6 months.

Patients in group A received traditional MT24 followed by a teletreatment including augmented reality MT. During the first four weeks, they performed exercises from the following categories with the intact limb in front of the mirror: observation of different positions, basic motor exercises, exercises using sensory stimuli. motor exercises using various objects and mental practice of phantom limb exercises. Patients were instructed to also perform the exercises with the phantom limb as soon as they perceived voluntary, pain-free movements of the phantom limb. During the last session, patients were given a tablet and a set of training materials. They received detailed verbal and written instructions on how to use the teletreatment The design and content of the teletreatment are described in detail in another publication ${ }^{22}$ The main funcionalities of the teletreatment included (1) monitoring of PLP. (2) digital exercise programmes using traditional MT (3) augmented (5) limb laterality recognition training (6) communication with the personal therapist and other patients and (7) background information on different topics. Patients were encouraged to use the teletreatment as often as they wished

the first four weeks but without further use of the materials were provided.

Patients in group C received the same amount and frequency of sensomotor exercises performed with the intact limb as those in groups sensomotor exercises with the intact limb at home, without handing out training materials.

\section{Measures}

Demographic characteristics such as date, reason and level of amputation were assessed through a self-developed questionnaire. In order to assess non-specific treatment effects, treatment expectancy and credibility of the treatment rationale after the patients had received their first allocated treatment session were scored using the credibility and expectancy questionnaire. ${ }^{25}$ The masked research assistant contacted all patients by phone at baseline and follow-up measurements at 4 weeks, 10 weeks and 6 months to guide patients through the questionnaires and to check completeness of data. The assistant asked patients not to reveal the assigned treatment during the measurement The primary outcome measures were the average intensity of PLP during the preceding week before outcome assessment on a Numeric Rating Scale (NRS) ${ }^{26}(0=n o$ pain, $10=$ worst pain), the frequency of PLP measured with a six-point scale $(0=n e v e r, 5=c o n s t a n t l y)$ and the duration of PLP measured with a seven-point scale ( $0=$ none, $6=$ constantly).

Secondary outcome measures were the different dimensions of PLP that were assessed through the German version of the Neuropathic Pain Specific Functional Scale Index rated on a 11 -point scale $\left(0=\right.$ no limitation, $10=$ complete limitation). ${ }^{30-32}$ Two additional questions about pain-related disturbances in sleep and mood were measured using an 11 -point NRS $(0=$ no limitation, $10=$ complete limitation). Qualtity of life was measured using the German version of the 5 -dimensional EuroQol questionnaire $-1(1=n 0$ problems, $5=$ unable to do/extreme problems) and a Visual Analogue Scale to score overall health ( $0=$ worst imaginable health; $100=$ best imaginable health). Index values are calculated from 0 (death) to 1 (full heath). The overall real

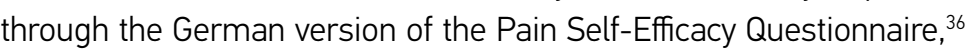
consisting of 10 items

In addition, patients were asked to provide the name, frequency and dose of pain medication at each follow-up measurement. Data regarding the frequency and type of teletreatment usage were automatically assessed by data logging. All patients were asked to register the frequency and type of self-delivered exercises and any adverse events in a log. Therapists were also asked to register the log. All completed questionnaires and logs were returned to the research assistant

Statistical analysis

The power calculation was based on the primary outcome, the average intensity in PLP of the preceding week on an 11-point NRS. For

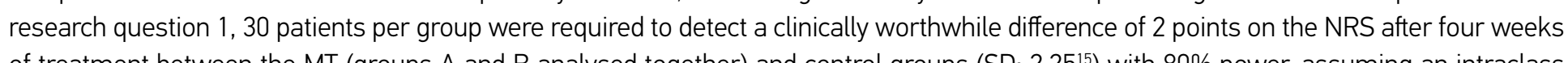
corelation (hesting within cente) of 0.10 and a $5 \%$ sigiftence 
105 participants (35 per group).

Statistical intention-to-treat analysis followed a predefined protocol21 using IBM SPSS Statistics for Windows (version 22.0). First, we checked whether the missing outcome data depended on baseline characteristics using Fisher's exact test for categorical variables and MannWhitney $U$ test for numerical variables. Variables significantly related to missingness were included in the linear mixed model, which uses all available data, deals with correlated data due to repeated measures and nesting of patients within centres, corrects for baseline differences and assumes missing data to be missing at random (MAR). ${ }^{30}$

Treatment effects on numerical outcomes were then assessed by including group, time, group ${ }^{*}$ time as the categorical variables. A random the main analysis was repeated with centre as between groups. Thereafter, the same mixed model analyses for the primary and secondary outcomes were repeated with correction for these differences in baseline demographics.

Next to intention-to-treat analyses, per-protocol analyses (with and without correction for baseline demographics) were performed. For research question 1 , patients in the MT group were considered as per protocol if at least 10 treatments were provided during the first four weeks. No further restrictions were made for palients in the control group. In addilion, patients in the teletrealment group who adhered to the protocol during the first four weeks and used at least 10 teletreatments with a minimal duration of 5 minutes during the following six weeks were considered as per protocol for research question 2 .

Predefined treatment interactions with gender (men vs. women) and post hoc with perceived length of the phantom limb (telescoping vs. normal) and type of PLP (cramping and unnatural position vs. other types) were performed as the literature suggests different effects of MT in these subgroups. ${ }^{39}$ Before these subgroup analyses were performed, we tested whether these were indeed significant effect modifiers

The frequency and duration ofLPP are frst descriplively analysed and visually displayed using bar graphs. In addition, to compare treatment ef che equivalent dily dosa (MED) was consideredstisically signifant was consideretstatistically significant.
RESULTS

In total. 75 patients were enrolled and randomized, of which 68 participants $(91 \%)$ were followed up at 4 weeks and $62(83 \%)$ at 10 weeks and 6 months. Figure 1 shows the reasons for ineligibility and discontinuation of treatment and illustrates the flow of participants.

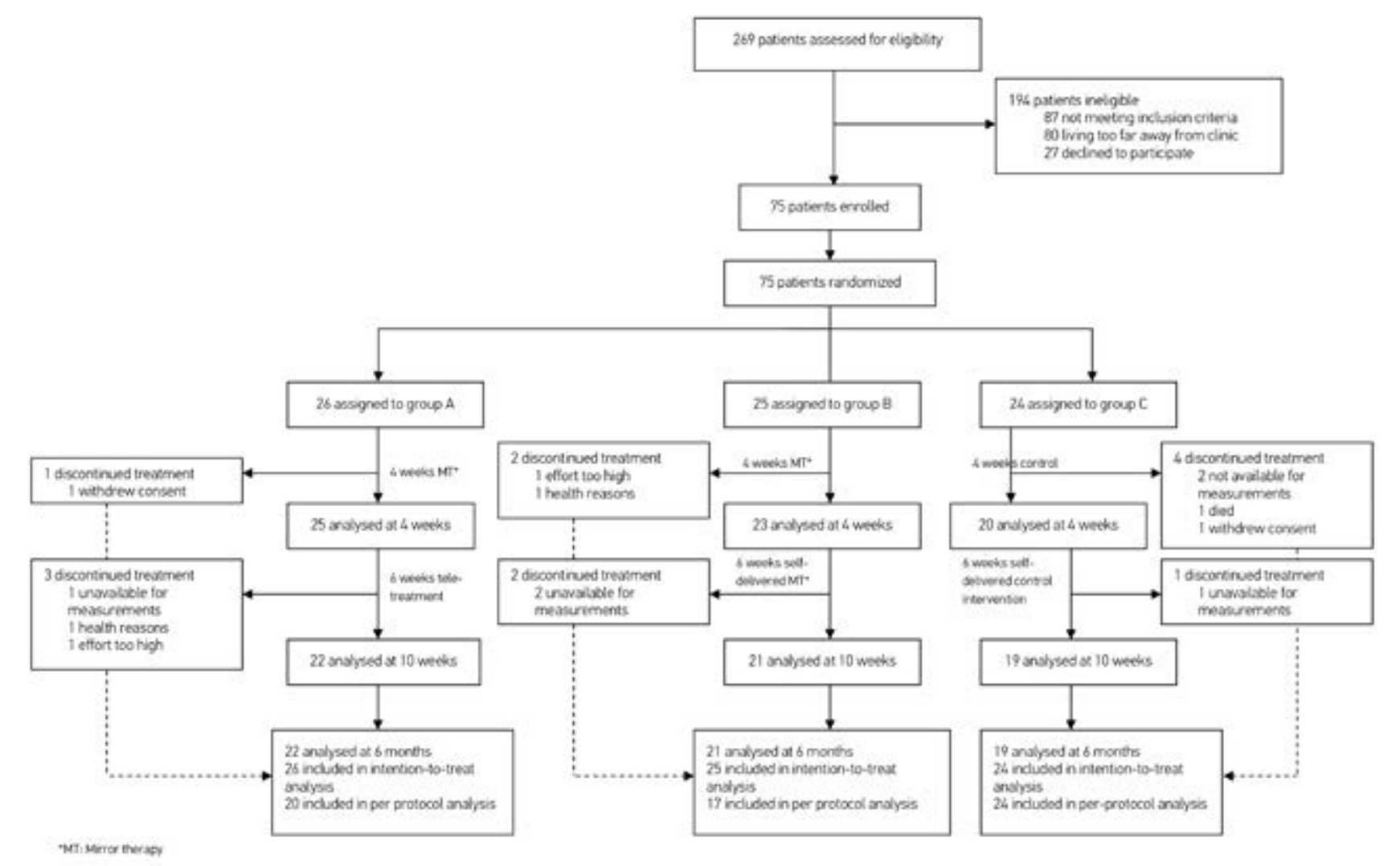

Figure 1. CONSORT flowchart of the PACT trial. 
Baseline differences between groups existed regarding gender, reason for amputation, prosthetic use, telescoping and perceived range of motion of the phantom limb (Table 1). Four patients in the MT group ( $A$ and B) and one patient in the control group (C) reported short events of transpiration in the beginning of the treatment.

Table 2 presents the observed means (SD) or \% (number of patients) per group and timepoint and the estimated treatment effects of MT (groups A and B) versus the control group (group C) at four weeks, corrected for baseline differences. During the first four weeks, 37 patients (73\%) in the MT group adhered to the predefined treatment protocol. Regarding the primary outcomes, the intention-to-treat analysis showed no significant treatment effect of MT over the control group on the average intensity of PLP in the preceding week at four weeks (treatment effect: $-1.2 ; 95 \%$ confidence interval (CI): -2.4 to $0.0 . P=0.054$ ) after correction for baseline differences. The effect size did also not reach the clinically worthwhile threshold specified in the trial protocol ( $>2.0$ points between groups). ${ }^{21}$

Table 1. Baseline characteristics of participants.

\begin{tabular}{|c|c|c|c|c|}
\hline Variable & Group $A^{*}(n=26)$ & Group $B^{*}(n=25)$ & Group $A+B^{\prime}(n=51)$ & Group $C^{d}(n=24)$ \\
\hline Age, mean (SD) & $59.7(16.1)$ & $62.5(11.4)$ & $61.1(13.9)$ & 61.0 (15.2) \\
\hline $\begin{array}{l}\text { Gender, male } \\
\text { Time post amputation, }\end{array}$ & $\begin{array}{l}80.8(21) \\
56.5(245-226.3)\end{array}$ & $\begin{array}{l}55.0 .0(14) \\
38.0(26-185.5)\end{array}$ & $\begin{array}{l}68.6(35) \\
38.0(25-219)\end{array}$ & $\begin{array}{l}70.8(17) \\
31.0(18.3-73.3)\end{array}$ \\
\hline $\begin{array}{l}\text { median (IQR), in months } \\
\text { Side of amputation, right }\end{array}$ & & & $52.9(27)$ & \\
\hline Level of amputation & & & & \\
\hline Foot & $7.6(2)$ & $0(0)$ & $4.0(2)$ & $0(0)$ \\
\hline Transtibial & $26.9(7)$ & $20.0(5)$ & $23.5(12)$ & $41.7(10)$ \\
\hline Knee disarticulation & $11.5(3)$ & $0(0)$ & $5.9(3)$ & $8.3(2)$ \\
\hline $\begin{array}{l}\text { Transsemoral } \\
\text { Hip disarticulation }\end{array}$ & $50.0(13)$ & $80.0(20)$ & $64.7(33)$ & 50.0 (12) \\
\hline $\begin{array}{l}\text { Hild disartriculation } \\
\text { Reason for amputation }\end{array}$ & $3.8(1)$ & & & \\
\hline Trauma & $38.5(10)$ & $32.0(8)$ & $35.3(18)$ & $29.2(7)$ \\
\hline Diabetes & & & & \\
\hline Dysvascular & 23.1 (6) & $24.0(6)$ & $23.5(12)$ & $41.7(10)$ \\
\hline $\begin{array}{l}\text { Tumour } \\
\text { Other }\end{array}$ & $15.4(4)$ & $20.0(5)$ & $17.6(9)$ & $42(1)$ \\
\hline Other (e.g. infection) & & & & \\
\hline
\end{tabular}

Table 1. (continued)

Variable Group $A^{*}(n=26) \quad$ Group $B^{*}(n=25) \quad$ Group $A+B^{\prime}(n=51) \quad$ Group $C^{(}(n=24)$

Prosthesis, yes

Usage time of prosthesis,
median (IRR), in hours/days

$88.0(22)$

Group $A+B(n)$

$70.8(17)$
$2.5(0-12)$

Perceived posture phantom
limb normal

$84.6(22)$

$88.0(22)$
$6.0(0.3-12$

$86.3(44)$
$6.0(1-14)$

Timb, normal

$69.2(18)$

$30.0(20)$

$74.5(38)$

91.7 (22)

Perceived range of motion
phantom limb

Very good

Good
Medium

Low

Nype of phantom pain
Buming

Buming

Cramping
Stabbing
Throbbing

Glowing

Cutting
Eltcectic shocks

lectric shocks
Pain because of unnatural

$20.0(5)$

21.6(11)

$33.3(8)$

$\begin{array}{rrr}7.7(2) & 0(0) & 3.9(2) \\ 11.5(3) & 20.0(5) & 15.7(8) \\ 1925 & 320(5) & 19.6(1)\end{array}$

$\begin{array}{lll}30.8(8) & 32.0(8) & 3.1 .4(16) \\ 19.2(5) & 20.0(5) & 19.6(10) \\ 30.8(8) & 28.0(7) & 29.4(15)\end{array}$

$12.5(3)$
$45.8(11)$

$35.3(18)$

$\begin{array}{ll}38.5(10) & 32.0(8) \\ 53.8(14) & 28.0(7) \\ 57.7(15) & 40.0(10)\end{array}$

$\begin{array}{ll}57.7(15) & 40.0(10) \\ 15.4(4) & 12.0(3) \\ 0(0) & 16.0(4) \\ 531(6) & 160(4)\end{array}$

$23.1(6)$
$53.8(14)$

Squeezing

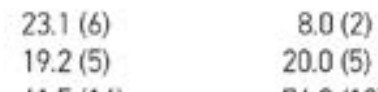

$16.0(4)$
$44.0(11)$
$0(0)$

$41.2(21)$
$49.0(25)$
$13.7(7)$

$13.7(7)$
$7.8(4)$
$19.6(10)$

$49.6(125)$
$3.9(2)$

$12.5(3)$
$8.3(2)$
$20.8(5)$

Work status, unemployed/

$15.7(8)$
$19.6(10)$
$68.6(35)$

$41.7(10)$

$29.2(7)$
50.0 (12)

$20.8(5)$
$1.25(3)$
$125(3)$

$12.5(3)$
$41.7(10)$
$0(0)$

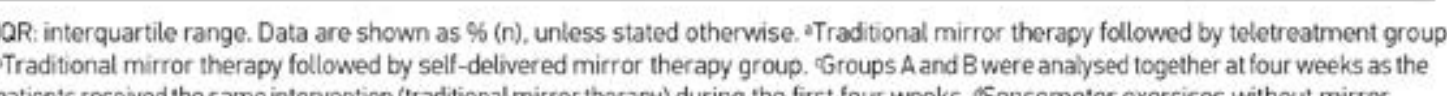

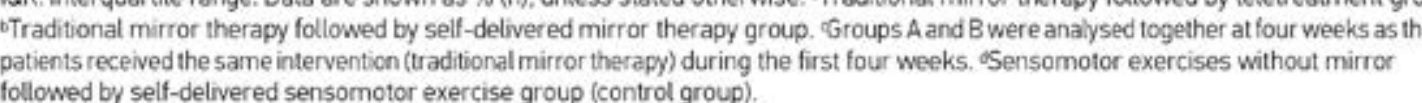


The frequency of PLP showed a positive change in all groups, with 22 patients (47\%) in the MT group and 6 patients (32\%) in the control group reporting improvement (Table 3) Particularly, patients who had constant pain benefitted (Tables 2 and 3, Supplementary Figure 3 , blue bar). Two patients in the MT group showed complete recovery of PLP.

Table 3. Frequency of phantom limb pain at baseline and after four weeks of intervention. Mirror therapy $(\mathrm{N}=47)$

\begin{tabular}{lccccc} 
& \multicolumn{2}{c}{} & & & \\
\cline { 2 - 3 } & Baseline & Four weeks & & Baseline & Four weeks \\
\hline Constantly & $44.7(21)$ & $25.5(12)$ & & $21.1(4)$ & $10.5(2)$ \\
Few per day & $25.5(12)$ & $23.4(11)$ & & $36.8(7)$ & $36.8(7)$ \\
Once per day & $6.4(3)$ & $2.1(1)$ & & $0(0)$ & $0(0)$ \\
Few per week & $8.5(4)$ & $25.5(12)$ & & $31.6(6)$ & $42.1(8)$ \\
$1-2$ per month & $14.9(7)$ & $19.1(9)$ & & $10.5(2)$ & $10.5(2)$ \\
Never & $0(0)$ & $4.3(2)$ & $0(0)$ & $0(0)$ \\
\hline
\end{tabular}

Dat from intention-to-treat analysis are shown as \% (n): "Groups A and B were analysed together a tour weeks as the patients received the

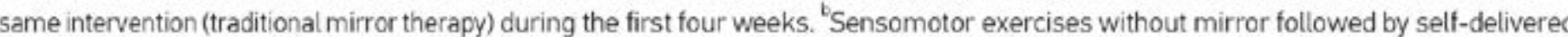
sensomotor exercises.

The duration of PLP improved in 17 patients (35\%) in the MT group and in 3 patients (16\%) in the control group. Again, the longer the pain estimating equation analyses showed no significant treatment effects between the groups recarding the frecuency and duration of PLP. The per-protocol analysis reveated a significant tieatri F
The secondary outcomes showed no significant effects in favour of any group. The per-protocol analysis revealed additional significant treatment effects of MT on pain-specific self-efficacy and global perceived effect (Supplementary Table 4).

The tests for effect modification showed a significant interaction of treatment with gender $(\mathrm{P}=0.045)$ and type of phantom pain (cramping and unnatural position; $P=0.040$ ), while interaction with telescoping was not significant $(P=0.367)$. The subgroup analyses suggested a significant and clinically worthwhile treatment effect of MT on the average PLP intensity in women ( $n=23$; treatment effect: $-2.4: 95 \% \mathrm{Cl}:-4.5$ to -0.4 ) but not in men ( $n=52$; treatment effect: $-0.3 ; 95 \% \mathrm{Cl}-1.7$ to 1.1). Similar significant and clinically worthwhile results on the average intensity of PLP were found for patients with telescoping ( $\mathrm{n}=19$; treatment effect: $-3.2 ; 95 \% \mathrm{Cl}-5.8$ to -0.6 ) and for patients perceiving a motor component (cramping or unnatural position) in PLP ( $\mathrm{n}=30$; treatment effect: $-3.1 ; 95 \% \mathrm{Cl}:-5.7$ to -0.5 ).

No reliable analysis of credibility and expectancy scores was possible due to too many missing values ( $n=50$ ), as many patients forgot to fill in the credibility and expectancy questionnaire after the first treatment Most of the patients used anti-epileptics and opioids and pain medication intake was reduced in the MT and control groups as shown in Supplementary Table 5.

At 10 weeks, 14 patients (54\%) in the traditional MT followed by the teletreatment group (group A) adhered to the predefined treatment protocol The main reasons for non-acherence were technical problems, insuffient instruction by therapists on how to use the platform and PLP already being sufficiently reduced by traditional MT during the first four weeks.

Table 4 shows the observed means (SD) or \% (n) per group and timepoint and the estimated treatment effects of the treatment groups at 10 weeks and 6 monhs corrected for baseline differences Regard ng the primary outcone intensity of PLP at 10 weeks and 6 months No statistically significant differences between the groups were found in the average intensity of PLP according to the intention-to-treat and per-protocol analyses.

The frequency of PLP showed a positive change at 10 weeks and 6 months in all groups at 6 months (Table 5). Patients who had constant pain 作 complete recovery of PLP at six months. Similar results were found for the duration of PLP with patients suffering from longer pain episodes and constant pain improving more than patients with shorter episodes of PLP.

At six months, 8 patients (36\%) in the teletreatment group. 14 patients (67\%) in the MT group and 5 patients (28\%) in the control groun showed a reduction in the duration of PLP episodes (Table L) The generalized estimating Ther the control $(P=0.019)$ and 
Regarding the secondary outcomes, patients in the teletreatment group showed significant and clinically worthwhile benefitsal over the control group regarding their overall health status at six months measured with the Visual Analogue Scale of the EuroQol questionnaire and both experimental groups showed significant and clinically worth- while effects' ${ }^{21}$ over the control group regarding the intrusion of PLP in daily life at all follow-up measurements (Table 4). The majority of secondary outcomes were not significantly different. The per-protocol analysis showed similar results (Supplementary Table 8). No significant interaction effects on the average intensity of PLP were found at 10 weeks and 6 months.

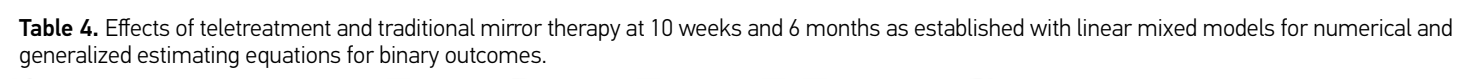

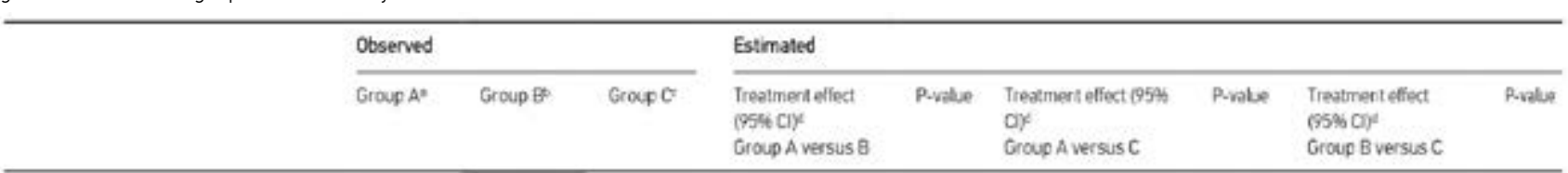

\begin{tabular}{|c|c|c|c|c|c|c|c|c|c|}
\hline \multirow{2}{*}{\multicolumn{10}{|c|}{ 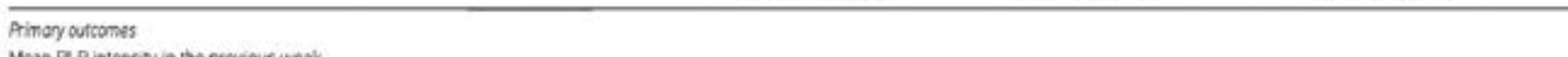 }} \\
\hline 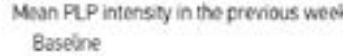 & & & & & & & & & \\
\hline 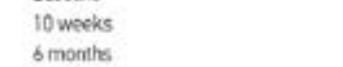 & 460199 & 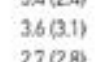 & $41028)$ & $\begin{array}{l}-0.3(-201014) \\
\end{array}$ & 0735 & $-03(-20.101 .5)$ & 0782 & $0.0(-18101.9)$ & 0.961 \\
\hline 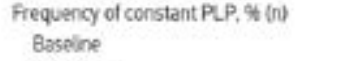 & & & & & & & & & \\
\hline 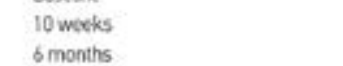 & 2736(6) & 10008 & 63019) & 190404084 & 0.378 & 20104010999 & 0.398 & $10020210677)$ & 0987 \\
\hline 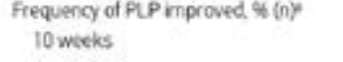 & 4800117) & 6500(3) & 579 (11) & 0502021017 & 0225 & $07021026)$ & 057 & 140.410507 & \\
\hline 6 memins & 5910139 & 700046 & 477.197) & $0.6021023)$ & 0.499 & 1.70510622 & 0.26 & 277071010.040 & 0.157 \\
\hline $\begin{array}{l}10 \text { wetens } \\
\text { ommontr }\end{array}$ & $300(9)$ & 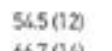 & $316(6)$ & 0.50101019 & 0.196 & $12(2035063)$ & 0781 & $26107109 / 4)$ & 0.163 \\
\hline \multirow{2}{*}{\multicolumn{10}{|c|}{ 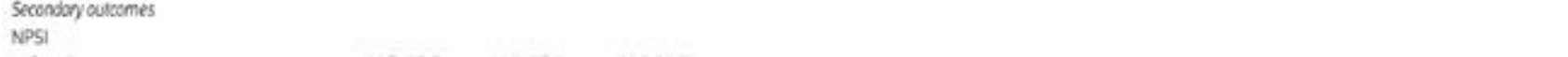 }} \\
\hline Bosenterte & & & & & & & & & \\
\hline 10wens & $228(132)$ & 181(193\}) & & $12(-8801011.1)$ & 0818 & $09(-110.0093)$ & 06864 & $-21(-12810877$ & 0708 \\
\hline 6 monilis & $194(133)$ & $141(20.6)$ & 156(129) & $-098-10.81090$ & 0.859 & $-0.2(-10.7510 .37\}$ & 0.966 & $07(-10101011.9)$ & 0503 \\
\hline
\end{tabular}

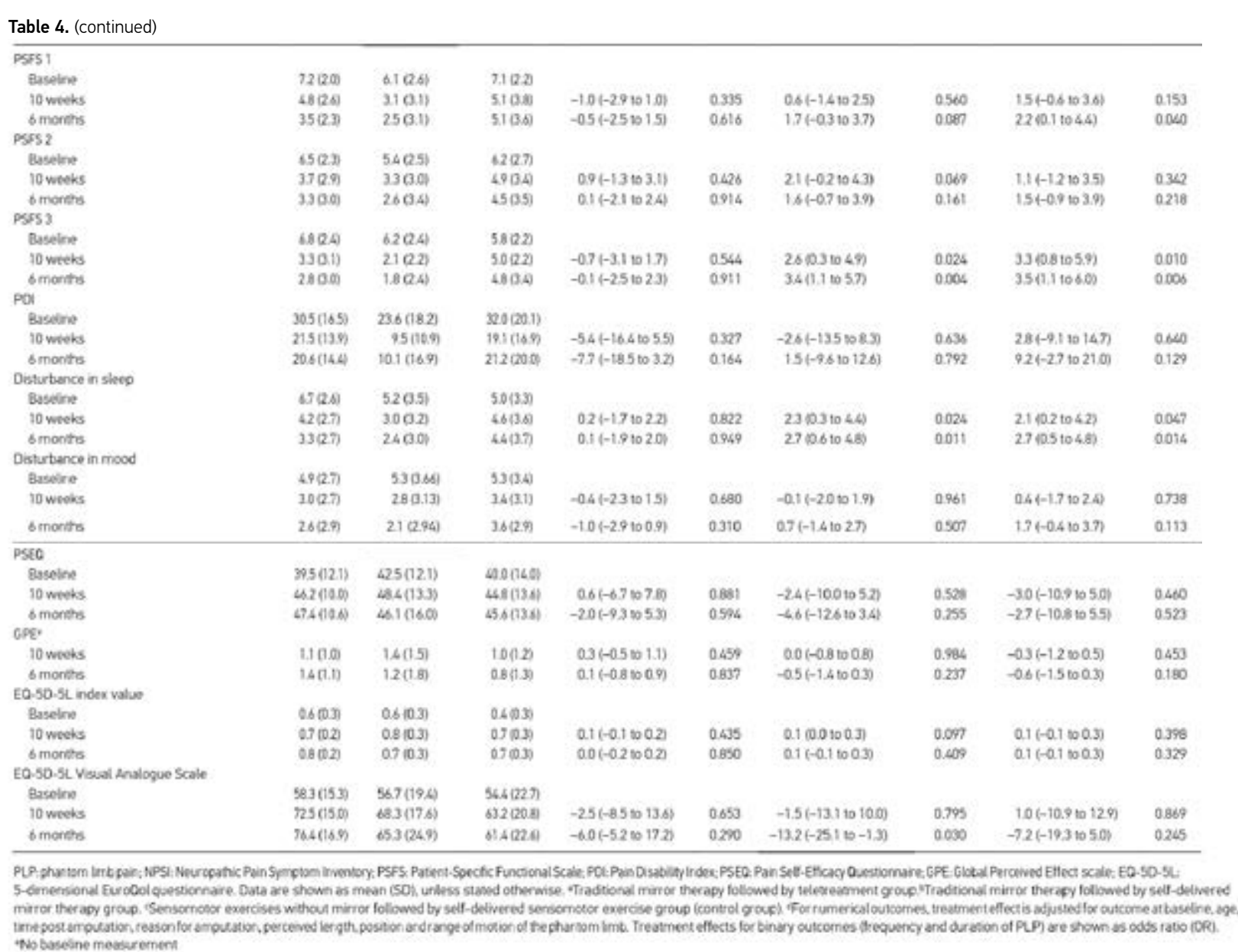


Table 5. Frequency of phantom limb pain at baseline and 10 weeks and 6 months of follow-up.

Group $A(\mathrm{~N}=22)^{\mathrm{a}} \quad \operatorname{Group} \mathrm{B}(\mathrm{N}=18)^{\mathrm{b}} \quad \operatorname{Group} \mathrm{C}(\mathrm{N}=16$

Baseline 10 weeks 6months Baseline 10 weeks 6months

$\begin{array}{llllllllll}\text { Constantly } & 50.0(11) & 27.3(6) & 31.8(7) & 33.3(6) & 10.5(2) & 10.5(2) & 25.0(4) & 6.3(1) & 12.5(2)\end{array}$

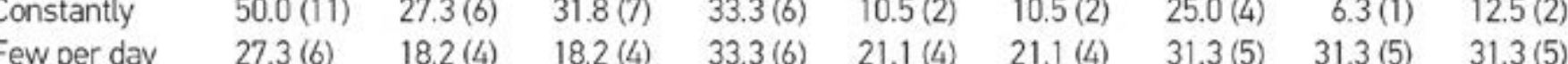

$\begin{array}{lrrrrrrrrr}\text { ew per day } & 27.3(6) & 18.2(4) & 18.2(4) & 33.3(6) & 21.1(4) & 21.1(4) & 31.3(5) & 31.3(5) & 31.3(5) \\ \text { Once per day } & 4.5(1) & 9.1(2) & 9.1(2) & 5.6(1) & 5.3(1) & 0(0) & 0(0) & 0(0) & 6.3(1)\end{array}$

$\begin{array}{lrrrrrrrrr}\text { Once per day } & 4.5(1) & 9.1(2) & 9.1(2) & 5.6(1) & 5.3(1) & 0(0) & 0(0) & 0(0) & 6.3(1) \\ \text { Few per week } & 9.1(2) & 31.8(7) & 18.2(4) & 11.1(2) & 21.1(4) & 15.8(3) & 37.5(6) & 37.5(6) & 31.3(5)\end{array}$

$\begin{array}{llllllllll}\text { few per week } & 9.1(2) & 31.8(7) & 18.2(4) & 11.1(2) & 21.1(4) & 15.8(3) & 37.5(6) & 37.5(6) & 31.3(5) \\ 1-2 \text { per month } & 9.1(2) & 13.6(3) & 22.7(5) & 16.7(3) & 31.6(6) & 36.8(7) & 6.3(1) & 25.0(4) & 18.8(3)\end{array}$

\begin{tabular}{lrrrrrrrrr}
$1-2$ per month & $9.1(2)$ & $13.6(3)$ & $22.7(5)$ & $16.7(3)$ & $31.6(6)$ & $36.8(7)$ & $6.3(1)$ & $25.0(4)$ & $18.8(3)$ \\
Never & $0(0)$ & $0(0)$ & $0(0)$ & $0(0)$ & $10.5(2)$ & $15.8(3)$ & $0(0)$ & $0(0)$ & $0(0)$ \\
\hline
\end{tabular}

Only complete data sets at six months are shown as \% (n): Traditional mirror therapy followed by teletreatment group. Traditiona sensomotor exercise group (control group).

DISCUSSION

A four-week intervention with traditional MT provided no statistically significant effects compared to sensomotor exercises without a mirror on the average intensity, frequency and duration of PLP at four weeks. Only the per-protocol analysis revealed significant effects of MT on the average intensity of PLP in the preceding week.

Subgroup analyses suggested significant and clinically worthwhile effects of traditional MT on the average intensity of PLP in women, patients with telescoping and in patients with a motor component regarding the type of PLP (cramping or unnatural position) at four weeks.

The use of a six-week teletrealment after four weeks of traditional MT did not provide significant additional benefit over self-delivered MT and self-delvered sensomer exesces wh
Methodological quality of the study

(2) (hessibility that the intervention itself did not work, his might also be explained by other aspects related to the population size and characteristics, the intervention, outcome measures and potential sources of bias.

Population size (power) and outcomes. The PACT trial is at present the largest randomized controlled trial on MT for patients with PLP using an intervention over 4-10 weeks and a long-term follow-up at 6 months. The three published controlled trials on MT with similar intervention periods ${ }^{15-17}$ had very small sample sizes ranging from $9^{15}$ to 18 amputees. ${ }^{16}$ Despite being the biggest trial so far, our study did not reach the calculated sample size and was therefore underpowered, which might explain why this study was unable to detect a significant but possibly worthwhile effect. The power calculation was based on a 2-point difference on the 11-point NRS regarding the average intensity of PLP in the preceding week between the groups. The effect sizes between the groups that were reported in the other controlled trials using similar intervention periods $15-17$ ranged from 12.915 to $27.2 \mathrm{~mm}^{17}$ on the Visual Analogue Scale. Compared to these studies, we found an estimated treatment effect on the average intensity of PLP of 12 in the preceding week between the groups on the NRS, which just did not reach statistical significance.

Looking back, the clinically worthwhile threshold of $>2.0$ points used for the power calculation might have been too strict as the study by Smith et al 43 defined a reduction of $1.15 \mathrm{~cm}$ on the Visual Analogue Scale as being clinically relevant for patients suffering from PLP. According to the Initiative on Methods, Measurement, and Pain Assessment in Clinical Trials (IMMPACT) recommendations 44 a $10 \%$ PLP. reduction in pain intensity reflects a minimally important change in chronic pain patients. In our study patients in the MT group showed a reduction in the average pain intensity of $263 \%$ (1.5 points on NRS) compared to $6.9 \%$ (0.4 points on NRS) in the control group at four weeks.

In addition, patients with PLP represent a very heterogeneous group with regard to the perceived intensity, frequency, duration and type of PLP. We also included people with infequent episodes of phant reveal any efect outcome. This study used the average intensity of PLP in the preceding week as a primary outcome, whereas other trials $15-17$ used the current level of PLP. A recent study $y^{45}$ suggested that a 
intensity or the number of pain episodes. The choice of the primary pain outcome measure in this study might have influenced the chance to demonstrate statistically significant effects.

Intervention characteristics. Prior clinical trials which used intervention periods shorter than one week did not show effects of $\mathrm{MT},{ }^{46,67}$ whereas studies using intervention periods of several weeks did. $15-17$ In line with these findings, this study demonstrates that only patients who adhered to the predefined treatment protocol and followed at least 10 sessions over four weeks showed a significant treatment effect on the primary outcome after four weeks.

A recent study by Griffin et al. ${ }^{\text {.8 }}$ suggested that patients with more severe PLP required up to 21 treatment sessions to obtain pain relief. Thus with more severe PLP.

Furthermore, the per-protocol analyses showed that almost half of the patients in the teletreatment group did not reach the predefined treatment intensity, which also could have influenced the contrast between the groups. It is possible that the teletreatment effect was less that might have led to lack of embodiment

Sources of bias. Potential sources of bias in this study might be related to spontaneous recovery of PLP, changes in medication intake, cointerventions, multiple testing and masking of patients and therapists.

Some studies suggest that PLP is decreasing over time without providing clear cut-offs for spontaneous recovery, 4.500 whereas other studies show no decrease or even an increase in PLP.50.51 In this study, spontaneous recovery of PLP is unlikely as the patients had an average time post amputation of about three years. As the majority of patients had no increase in pain medication it is unlikely that the effects on PLP were caused by chances in medication intake. As co-interventions were not monitored, we do not know whether patients in the control group. for example also had MT or other co-interventions after the first four weeks, which might have influenced the contrast between the groups. In addition, this study assessed many secondary outcomes resulting in multiple statistical testing, which in turn increases the probality false-positive results In this study, the statistician and the research a However, it was not possible to mask patients and therapists, which might have influenced the results.
Effects in relation to patient characteristics

A prior stud ${ }^{39}$ shows that MT is more effective in patients reporting motor qualities in their phantom limb sensation such as cramping or an unnatural postion, which is also suggested by our study. This might be explained by the hypothesis that MT targets the maladaptive neuroplastic changes that correlate with the degree of PLP and the ability to move the phantom limb. 4.6 .7 Recent studies have demonstrated that mental practice and MT are able to restore primary sensory and motor cortex organization ${ }^{10.11}$ and are able to improve voluntary motor control over the phantom limb, 12.46 which in turn might reduce PLP.

Furthermore, the study by Foell et al." suggests that MT is less effective in patients with a telescoping phantom, which was not supported by our results. The study by Schmalzl and Ehrsson ${ }^{52}$ showed that the perceived length of the phantom limb can dynamically be manipulated by congruent visuo-tactile information and thereby revoking the telescoping sensation. This altered telescoping sensation could result in a reduction of PLP, as the perception of telescoping seems to be positively correlated with the intensity in PLP.53 Similar results were found in lleled by an effect on the telescoping sensation and the perceived posture of the phantom (closed fist).

In addition to the existing literature, our subgroun analyses suggest that women benefit more from the intervention than men This could Then that women might be more capable of engaing in the mirror illusion and hence achieve higher levels of body ownership of the mirrored limb. The batter is thought to be positively correlated with activation of the deprived sensorimotor cortex and reduction in PLP. 55

However, any conclusions that are drawn from subgroup analyses with a small sample size need to be interpreted with caution ${ }^{56}$ and clear evidence for these assumptions is missing as the precise working mechanism of MT remains speculative.

Implications for research and clinical practice

Based on the literature ${ }^{42}$ and our results, it is evident that applying a complex intervention to a heterogeneous patient group is challenging. Future research should focus on identifying eligible patients for MT as several subtypes of patients showed better response to treatment as suggested by our subgroup analysis

In addition to selecting eligible patients, the intervention should also be tailored to the characteristics and preferences of patients with PLP.

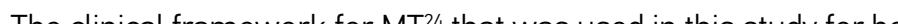

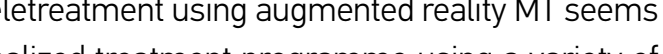


exercises from the different categories of our framework is essential as some patients gain less benefit from basic motor exercises only. We however only found a small effect of the framework in this study, which might be explained by various limitations described above. Furthermore, future studies should focus on identifying appropriate primary outcome measures for patients with PLP that match the individual perception of the phantom limb. It would also be useful to develop a questionnaire that is able to assess patient engagement in and the vividness of the mirror illusion to select eligible patients.

Recently, augmented and virtual reality approaches have been proposed for patients with PLP who did not respond to the traditional MT approach ${ }^{19}$ In our study the novel teletreatment using augmented reality MT had no additional effects compared to self-delivered traditional MT and limited positive effects on secondary outcomes compared to the control group. Thus, the additional value of such approaches needs further investigation.

Clinical Messages
- Four weeks of MT had small but non-significant effects on the
duration and average intensity of PLP.
- The clinical framework that was evaluated in this study seems to
be feasible and can be used to personalize MT in daily care.
- The teletreatment showed no additional effects.

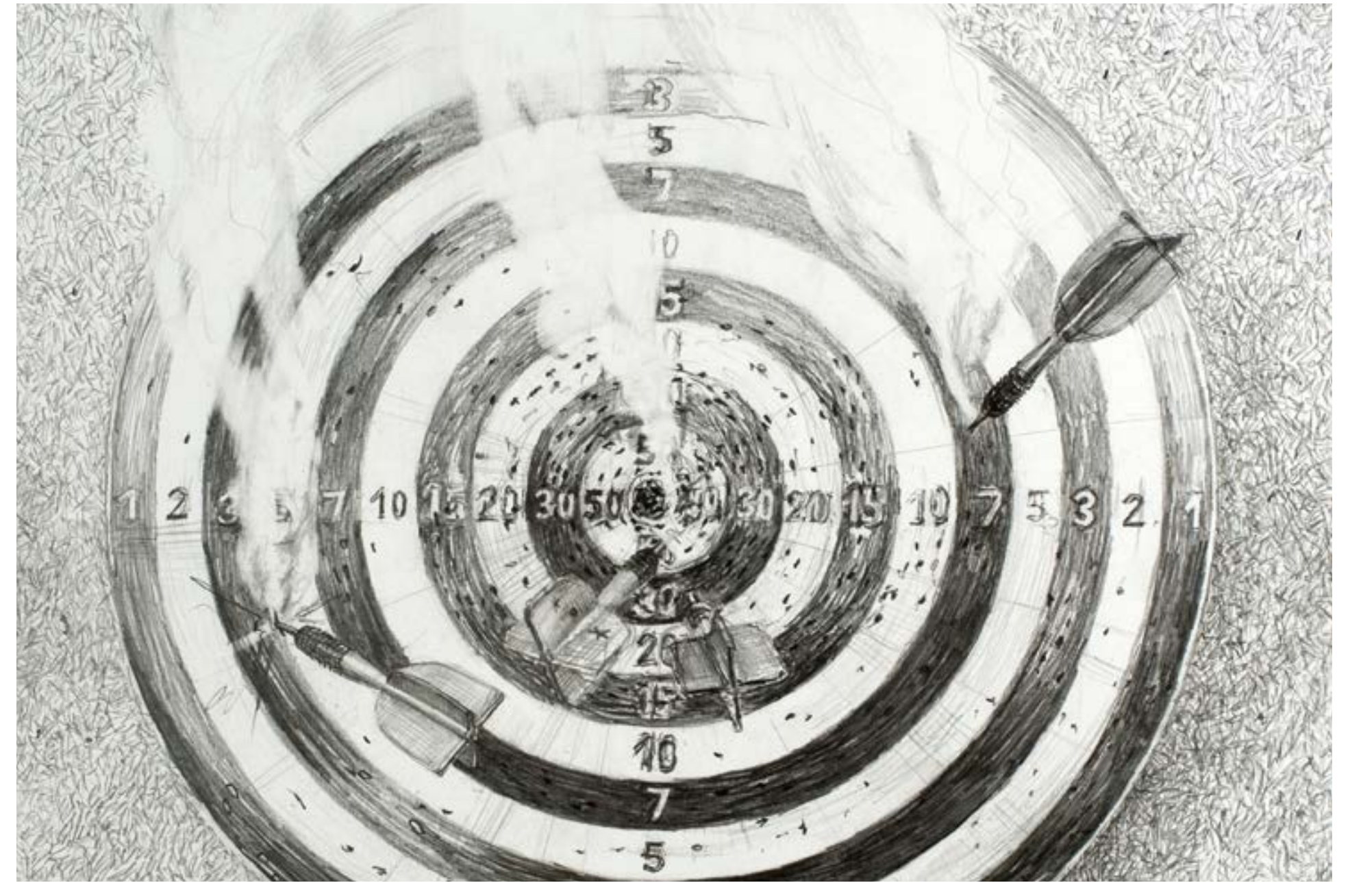




\section{REFERENCES}

Alviar MJ, Hale T and Dungca M. Pharmacologic interventions for treating phantom limb pain. Cochrane Database Syst Rev 2016; 12: CD006380.

Aiyer R, Barkin RL, Bhatia A, et al. A systematic review on the treatment of phantom limb pain with spina cord stimulation. Pain Manag 2017; 7: 59-69.

Johnson MI, Mulvey MR and Bagnall AM. Transcutaneous electrical nerve stimulation (TENS) for phantom pain and stump pain following amputation in adults. Cochrane Database Syst Rev 2015; 8: CD007264. Flor $\mathrm{H}$, Elbert T, Knecht S, et al. Phantom-limb pain as a perceptual correlate of cortical reorganization following arm amputation. Nature 1995; 375: 482-484.

Knecht S, Henningsen $\mathrm{H}$, Hohling $\mathrm{C}$, et al. Plasticity of plasticity? Changes in the pattern of perceptual correlates of reorganization after amputation. Brain 1998; 121(Pt 4): 717-724.

Lotze M, Grodd W, Birbaumer N, et al. Does use of a myoelectric prosthesis prevent cortical reorganization and phantom limb pain? Nat Neurosci 1999; 2: 501-502

7. Raffin E, Richard N, Giraux P, et al. Primary motor cortex changes after amputation correlate with phantom limb pain and the ability to move the phantom limb. Neuroimage 2016; 130: 134-144. Makin TR, Scholz J, Filippini N, et al. Phantom pain is associated with preserved structure and function in the former hand area. Nat Commun 2013; 4: 1570.

9. Bekrater-Bodmann R, Schredl M, Diers M, et al. Post-amputation pain is associated with the recall of an impaired body representation in dreams - results from a nation-wide survey on limb amputees. PLoS ONE 2015; 10: e0119552.

10. Maclver K, Lloyd DM, Kelly S, et al. Phantom limb pain, cortical reorganization and the therapeutic effect of mental imagery. Brain 2008; 131: 2181-2191.

11. Foell J, Bekrater-Bodmann R, Diers M, et al. Mirror therapy for phantom limb pain: brain changes and the role of body representation. Eur J Pain 2014; 18: 729-739.
2. Ramachandran VS, Rogers-Ramachandran D and Cobb S. Touching the phantom limb. Nature 1995; 377 489-490

13. Barbin J, Seetha V, Casillas JM, et al. The effects of mirror therapy on pain and motor control of phantom limb in amputees: a systematic review. Ann Phys Rehabil Med 2016; 59: 270-275.

14. Rothgangel AS, Braun SM, Beurskens AJ, et al. The clinical aspects of mirror therapy in rehabilitation: a systematic review of the literature. Int J Rehabil Res 2011;34: 1-13.

15. Moseley GL. Graded motor imagery for pathologic pain: a randomized controlled trial. Neurology 2006; 67:2129-2134.

16. Chan BL, Witt R, Charrow AP, et al. Mirror therapy for phantom limb pain. N Engl J Med 2007; 357: 22062207.

17. Finn SB, Perry BN, Clasing JE, et al. A randomized, controlled trial of mirror therapy for upper extremity phantom limb pain in male amputees. Front Neurol 2017; 8: 267.

18. Schmalzl L, Ragno $\mathrm{C}$ and Ehrsson HH. An alternative to traditional mirror therapy: illusory touch can reduce phantom pain when illusory movement does not. Clin J Pain 2013; 29: e10-e18.

19. Ortiz-Catalan M, Guethmundsdottir RA, Kristoffersen MB, et al. Phantom motor execution facilitated by

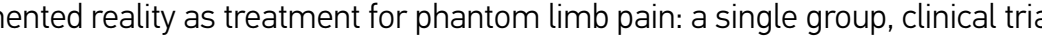
in patients with chronic intractable phantom limb pain. Lancet 2016; 388: 2885-2894.

20. Agostini M, Moja L, Banzi R, et al. Telerehabilitation and recovery of motor function: a systematic review and meta-analysis. J Telemed Telecare 2015; 21: 202-213.

21. Rothgangel AS, Braun S, Schulz RJ, et al. The PACT trial: PAtient Centered Telerehabilitation: effectiveness of software-supported and limb amputation: protocol of a multicentre randomised controlled trial. J Physiother 2015; 61: 42.

22. Rothgangel A, Braun S, Smeets R, et al. Design and development of a telerehabilitation platform for patients with phantom limb pain: a user-centered approach. JMIR Rehabil Assist Technol 2017; 4: e2. 
23. Jensen MP and McFarland CA. Increasing the reliability and validity of pain intensity measurement in chronic pain patients. Pain 1993; 55: 195-203.

24. Rothgangel A, Braun S, de Witte L, et al. Development of a clinical framework for mirror therapy in Devilly GJ and Borkore Behav Ther Exp Psychiatry 2000; 31: 73-86,

Ineme measures for chronic pain clinical trials: IMMPACT recommendations. Pain 2005; 113: 9-19.

27. Sommer $\mathrm{C}$, Richter $\mathrm{H}$, Rogausch JP, et al. A modified score to identify and discriminate neuropathic pain: a study on the German version of the Neuropathic Pain Symptom Inventory (NPSI). BMC Neurol 2011;11:104

28. Bouhassira D, Attal N, Fermanian J, et al. Development and validation of the neuropathic pain symptom inventory. Pain 2004; 108: 248-257.

29. Heldmann P, Schoettker-Koeniger T and Schaefer A. Cross-cultural adaption and validity of the patien specific functional scale. Int J Health Prof 2015; 2: 73-82.

Pollard CA. Preliminary validity study of the pain disability index. Percept Mot Skills 1984; 59:974. Dillmann U, Nilges P, Saile H, et al. Assessing disability in chronic pain patients. Schmerz 1994; 8: 100-

32. Tait RC, Pollard CA, Margolis RB, et al. The pain disability index: psychometric and validity data. Arch Phys Med Rehabil 1987; 68: 438-441.

3. Hinz A, Kohlmann T, Stobel-Richter Y, et al. The quality of life questionnaire EQ-5D-5L: psychometric properties and normative values for the general German population. Qual Life Res 2014; 23: 443-447.

34. Herdman M, Gudex C, Lloyd A, et al. Development and preliminary testing of the new five-level version of EQ-5D (EQ-5D-5L). Qual Life Res 2011; 20: 1727-1736.
35. Kamper SJ, Ostelo RW, Knol DL, et al. Global perceived effect scales provided reliable assessments of health transition in people with musculoskeletal disorders, but ratings are strongly influenced by current status. J Clin Epidemiol 2010; 63: 760-766.

36. Mangels M. Schwarz S. Sohr G et al. Der Fragebogen zur Erfassung der schmerzspezifischen Selbstwirksamkeit (FESS). Diagnostica 2009; 55: 84-93.

Nicholas MK. The pain self-efficacy questionnaire: taking pain into account. Eur J Pain 2007; 11: 153-163. Verbeke $G$ and Molenberghs $G$. Linear mixed models for longitudinal data. New York: Springer, 2000, Sumitani M, Miyauchi S, McCabe CS, et al. Mirror visual feedback alleviates deafferentation pain, depending on qualitative aspects of the pain: a preliminary report. Rheumatology 2008; 47: 1038-1043. National Institute for Care and Health Excellence. What are the equivalent doses of oral morphine to other oral opioids when used as analgesics in adult palliative care? https://www.evidence.nhs.uk Chen P, Lin KC, Liing RJ, et al. Validity, responsiveness, and minimal clinically important difference of EQ5D-5L in stroke patients undergoing rehabilitation. Qual Life Res 2016; 25: 1585-1596.

42. Craig P, Dieppe P, Macintyre S, et al. Developing and evaluating complex interventions: the new Medical Research Council guidance. BMJ 2008; 337: a 1655.

43. Smith DG, Ehde DM, Hanley MA, et al. Efficacy of gabapentin in treating chronic phantom limb and residual limb pain. J Rehabil Res Dev 2005; 42: 645-654.

44. Dworkin RH, Turk DC, Wyrwich KW. et al. Interpreting the clinical importance of treatment outcomes in chronic pain clinical trials: IMMPACT recommendations. J Pain 2008; 9: 105-121.

45. Richardson $\mathrm{C}$ and Kulkarni J. A review of the management of phantom limb pain: challenges and solutions. J Pain Res 2017; 10: 1861-1870.

46. Brodie EE, Whyte A and Niven CA. Analgesia through the looking-glass? A randomized controlled trial investigating the effect of viewing a 'virtual' limb upon phantom limb pain, sensation and movement. Eur J Pain 2007; 11: 428-436. 
47. Tilak M, Isaac SA, Fletcher J, et al. Mirror therapy and transcutaneous electrical nerve stimulation for

management of phantom limb pain in amputees - a single blinded randomized controlled trial. Physiother

Res Int 2016; 21: 109-115.

48. Griffin SC, Curran S, Chan AWY, et al. Trajectory of phantom limb pain relief using mirror therapy: retro-

spective analysis of two studies. Scand J Pain 2017; 15: 98-103.

Houghton AD, Nicholls G, Houghton AL, et al. Phantom pain: natural history and association with

rehabilitation. Ann R Coll Surg Engl 1994; 76: 22-25.

50. Nikolajsen L, Ilkjaer S, Kroner $\mathrm{K}$, et al. The influence of preamputation pain on postamputation stump and phantom pain. Pain 1997; 72: 393-405.

Richardson C, Crawford K, Milnes K, et al. A clinical evaluation of postamputation phenomena including phantom limb pain after lower limb amputation in dysvascular patients. Pain Manag Nurs 2015; 16:561-569. Schmalzl L and Ehrsson HH. Experimental induction of a perceived 'telescoped' limb using a full-body llusion. Front Hum Neurosci 2011; 5: 34

53. Flor $\mathrm{H}$, Nikolajsen $\mathrm{L}$ and Jensen TS. Phantom limb pain: a case of maladaptive CNS plasticity? Nat Rev Neurosci 2006; 7: 873-881.

Cof Ortiz-Catalan M, Sander N, Kristoffersen MB, eat Treatment of phat

Schaefer M, Flor H, Heinze HJ, et al. Morphing the body: illusory feeling of an elongated arm affects somatosensory homunculus. Neuroimage 2007; 36: 700-705

56. Wang R, Lagakos SW, Ware JH, et al. Statistics in medicine - reporting of subgroup analyses in clinical trials. N Engl J Med 2007; 357: 2189-2194 


\section{APPENDIX CHAPTER 6}

SUPPLEMENTARY FIGURES AND TABLES 


\section{Global Perceived Effect scale}

Please indicate below to which extent your phantom limb pain has changed through the treatment:

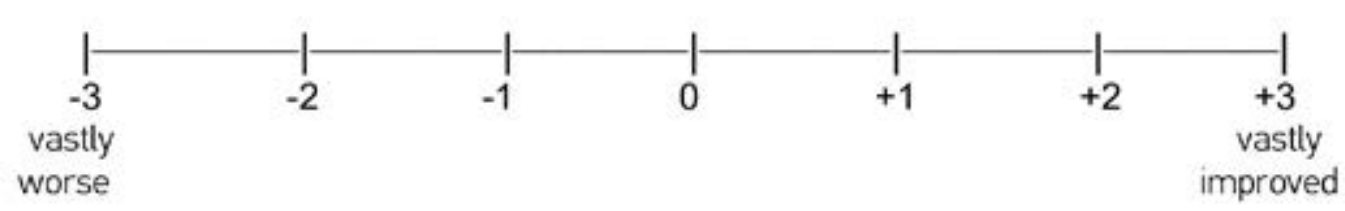

Figure 1. Teletreatment using augmented reality mirror therapy: Movements of the intact limb are filmed by conventional camera in the tablet and mirrored on the tablet screen. Virtual objects can be added to the exercise program.

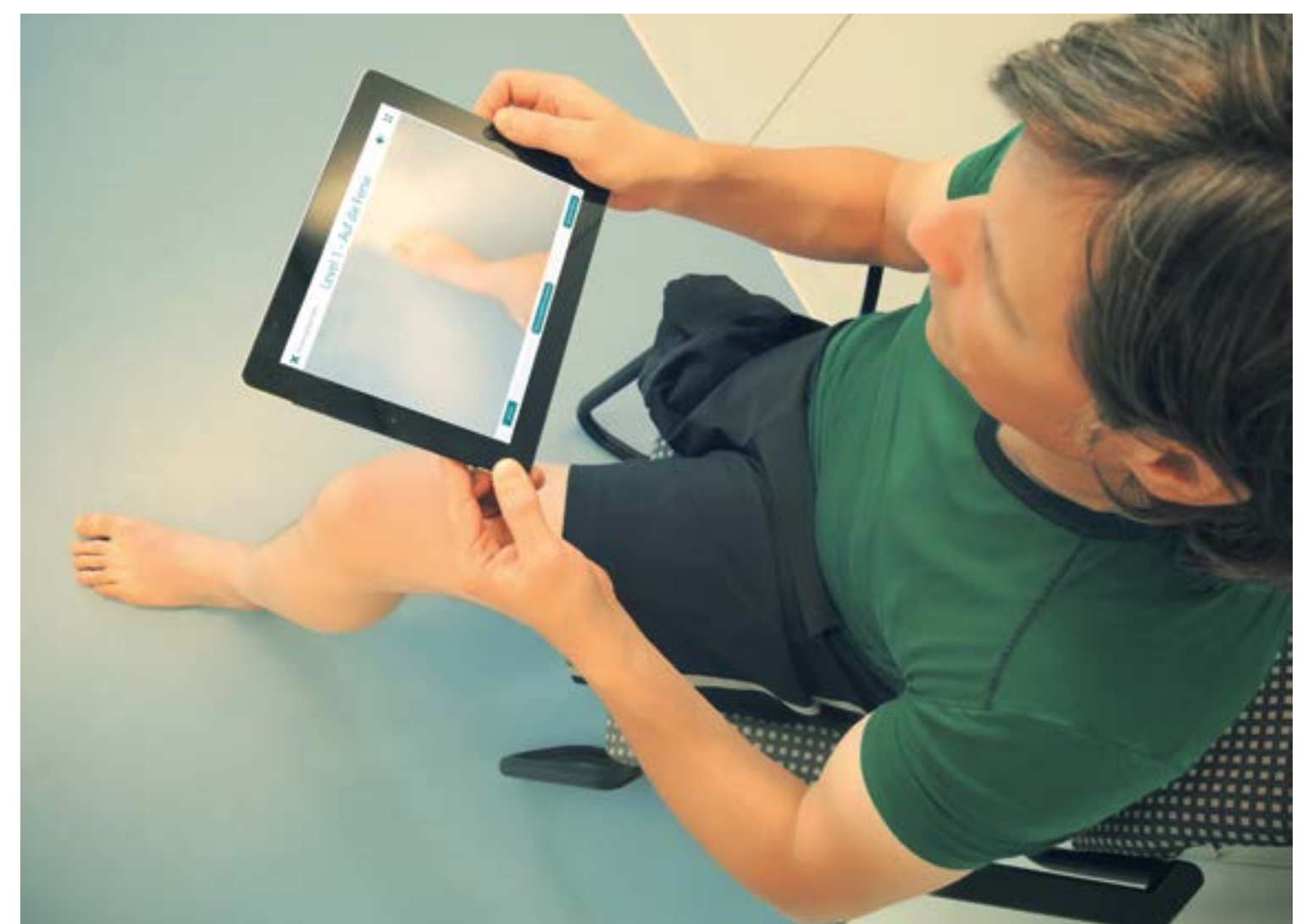


Figure 3. Frequency of phantom limb pain measured at baseline and 4 weeks

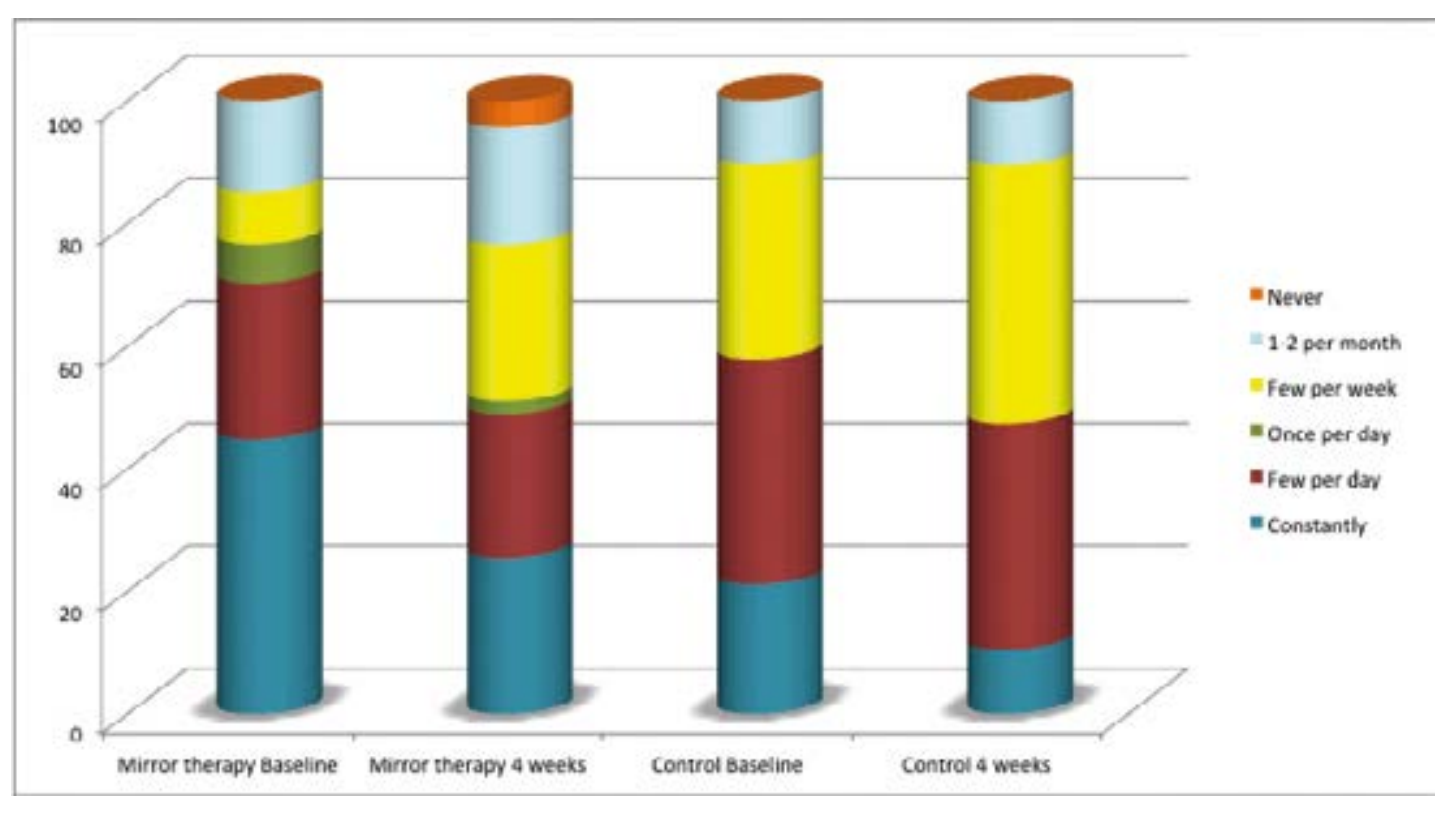

Mirror therapy: Group A and B were analysed together as patients received the same intervention (traditional mirror therapy) during the first 4 weeks. Control group: Sensomotor exercises without mirror followed by self-delivered sensomotor exercises.
Figure 4. Frequency of phantom limb pain measured at baseline, 10 weeks and 6 months

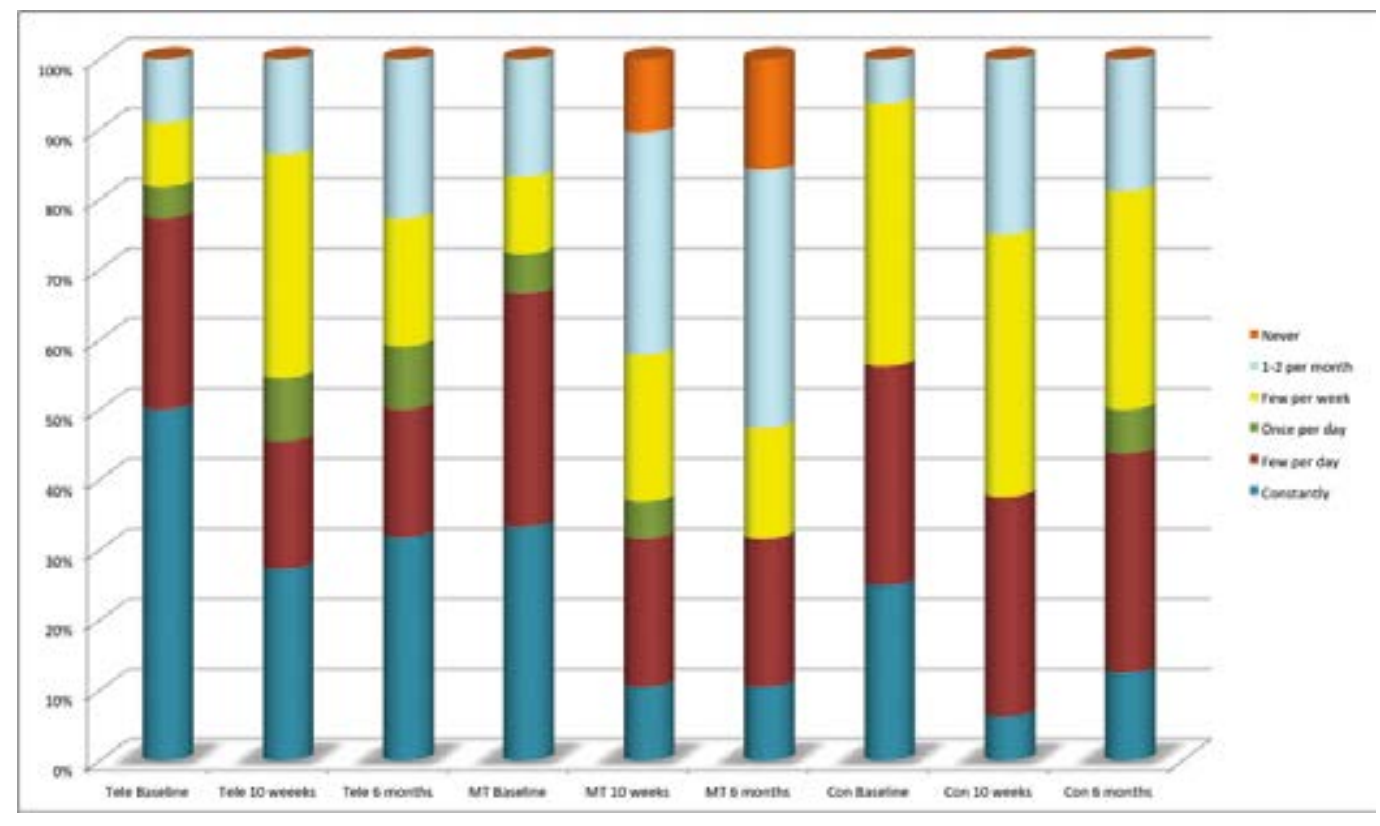

Tele:Traditional mirror therapy followed by teletreatment group (Group A); MT: Traditional mirror therapy followed by self-delivered mirror therapy group (Group B); Con: Sensomotor exercises without mirror followed by self-delivered sensomotor exercises group (control group C). 


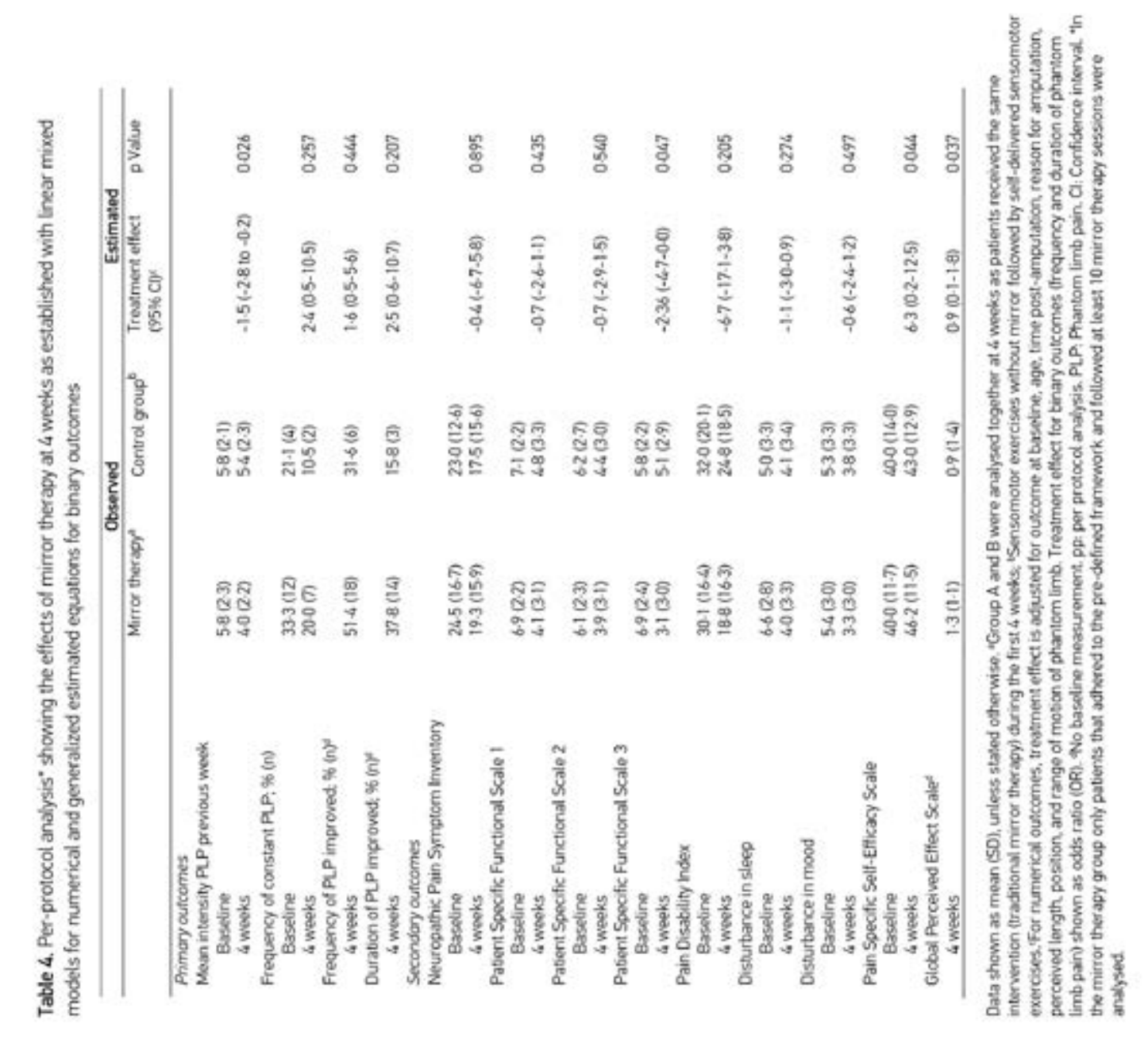

Table 5. Changes in pain medication of patients in the mirror therapy and control group at baseline and different

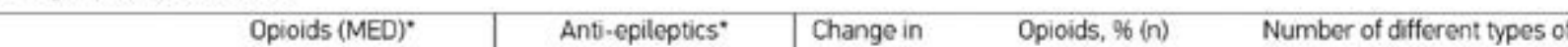

\begin{tabular}{|c|c|c|c|c|c|c|c|c|c|}
\hline & \multicolumn{2}{|c|}{ Opioids (MED)" } & \multicolumn{2}{|c|}{ Ant-epileptics" } & \multirow{2}{*}{$\begin{array}{l}\text { Change in } \\
\text { medication } \\
\text { intake }\end{array}$} & \multicolumn{2}{|c|}{ Opioids, $96(n)$} & \multicolumn{2}{|c|}{$\begin{array}{l}\text { Number of different types of } \\
\text { medication used, } \%(n)\end{array}$} \\
\hline & $\begin{array}{l}\text { Mirror } \\
\text { therapy }\end{array}$ & Control & $\begin{array}{l}\text { Mirror } \\
\text { therapy }\end{array}$ & Controle & & $\begin{array}{l}\text { Mirror } \\
\text { therapy }\end{array}$ & Control & $\begin{array}{l}\text { Mirror } \\
\text { therapy }\end{array}$ & Control \\
\hline $\begin{array}{l}\text { Baseline } \\
\% \text { (n) } \\
\text { Total daily dose. } \\
\text { mean (SD) } \\
4 \text { weeks }\end{array}$ & $\begin{array}{l}100(16) \\
65.8(47.8)\end{array}$ & $\begin{array}{l}100(10)(10) \\
59.8(70.5)\end{array}$ & $100(23)$ & $100(12)$ & & & & & \\
\hline 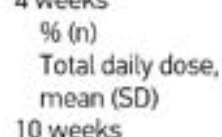 & $\begin{array}{l}75.0(12) \\
54.5(5.44)\end{array}$ & $\begin{array}{l}80.0(8) \\
46.3(57.8)\end{array}$ & 82. (19) & $91.7(11)$ & $\begin{array}{l}\text { No change } \\
\text { Less } \\
\text { More }\end{array}$ & $\begin{array}{l}41.2(7) \\
41.2(7) \\
17.6(3)\end{array}$ & $\begin{array}{l}41.7(5) \\
417(5) \\
16.7(2)\end{array}$ & $\begin{array}{l}69.0(20) \\
27.6(8) \\
3.4(1)\end{array}$ & $\begin{array}{l}75.0(12) \\
25.0(4) \\
0(0)\end{array}$ \\
\hline $\begin{array}{l}96(n) \\
\text { Total daliy dose, } \\
\text { mean (SD) } \\
6 \text { months }\end{array}$ & $\begin{array}{l}62.5(10) \\
40.5(42.2)\end{array}$ & $\begin{array}{l}60.0 .0(6) \\
332(49.0)\end{array}$ & $87.0(20)$ & $83.3(10)$ & $\begin{array}{l}\text { No change } \\
\text { Less } \\
\text { More }\end{array}$ & $\begin{array}{l}33.3(6) \\
55.6(10) \\
11.1(2)\end{array}$ & $\begin{array}{l}25.0(3) \\
44.7(5) \\
33.3(4)\end{array}$ & 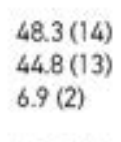 & 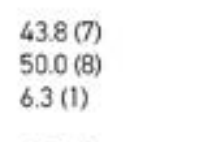 \\
\hline $\begin{array}{l}90 \text { (n) } \\
\text { Total daily dose, } \\
\text { mean (SO) }\end{array}$ & $\begin{array}{l}56.3(9) \\
48.3(69.8)\end{array}$ & 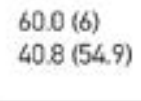 & $7.3 .9(17)$ & $75.0(9)$ & $\begin{array}{l}\text { No change } \\
\text { Less } \\
\text { More }\end{array}$ & $\begin{array}{l}22.2(4) \\
61.1(11) \\
16.7(3)\end{array}$ & $\begin{array}{l}27.3(3) \\
63.6(1) \\
9.1(1)\end{array}$ & $\begin{array}{l}48.3(14) \\
51.7(15) \\
0(0)\end{array}$ & $\begin{array}{l}53.3(8) \\
46.7(7) \\
0(0)\end{array}$ \\
\hline
\end{tabular}

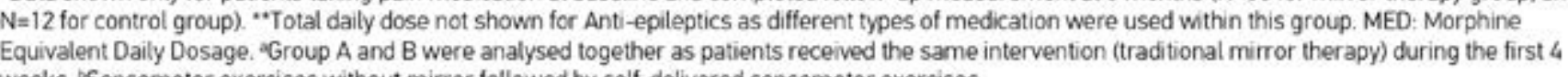

weeks. S.Sensomotor exercises without mirror followed by selt-delivered sensomotor exercises. 
Table 8. Per-protocol analysis showing the effects of teletreatment and traditional mirror therapy at 10 weeks and 6 months as flor numerical and generalzed estimated equations for binary outcomes

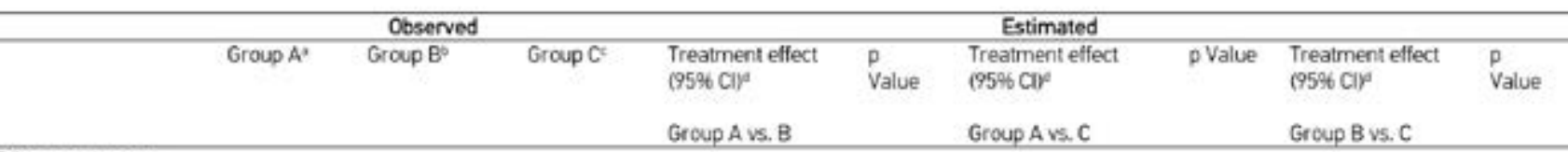

\begin{tabular}{|c|c|c|c|c|c|c|c|c|c|}
\hline 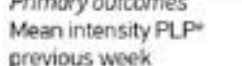 & & & & & & & & & \\
\hline $\begin{array}{l}\text { Bassenen } \\
100 \text { ereks }\end{array}$ & $\begin{array}{l}6.2(1.9) \\
4.7(2.1)\end{array}$ & $\begin{array}{l}55(2.7) \\
35(322)\end{array}$ & $\begin{array}{l}58(2.1) \\
412.6)\end{array}$ & & & & & & \\
\hline $\begin{array}{l}6 \text { months } \\
\text { Epeoponof constrot }\end{array}$ & $44(26)$ & $28(30)$ & 452.88 & $-1 \cdot 1(-34-12)$ & 0.343 & $06(-17-28)$ & 0.616 & $1.7(-04-3.8)$ & 0.121 \\
\hline$P L P, \%(n)$ & & & & & & & & & \\
\hline 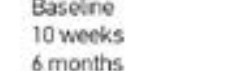 & 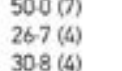 & $\begin{array}{l}1863 \\
118082 \\
1252\end{array}$ & 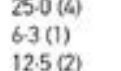 & $\begin{array}{l}1.2(0.1-15.0) \\
07(0.150)\end{array}$ & $\begin{array}{l}0.075 \\
0.74\end{array}$ & $\begin{array}{l}1.9(0.1-35.9) \\
07(0.1-52)\end{array}$ & 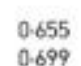 & $\begin{array}{l}1.6(0.1-36.0) \\
090(0.1-6.6)\end{array}$ & $\begin{array}{l}0.771 \\
0.954\end{array}$ \\
\hline 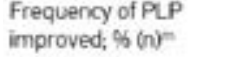 & & & & & & & & & \\
\hline $\begin{array}{l}\text { io weens } \\
6 \text { months }\end{array}$ & 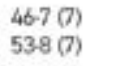 & 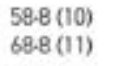 & $\begin{array}{l}577(1) \\
67.1(8)\end{array}$ & $\begin{array}{l}0.5(0.122) \\
08(02-346)\end{array}$ & $\begin{array}{l}0.399 \\
0.770\end{array}$ & $\begin{array}{l}0.50 .1-2.6) \\
20(0.5-9.1)\end{array}$ & $\begin{array}{l}0.434 \\
0.347\end{array}$ & $\begin{array}{l}1.0(0.2-2.6) \\
2.5(0.5-118)\end{array}$ & $\begin{array}{l}0.979 \\
0.234\end{array}$ \\
\hline 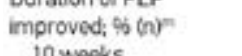 & & & & & & & & & \\
\hline $\begin{array}{l}10 \text { weens } \\
6 \text { 6months }\end{array}$ & $\begin{array}{l}40(6) \\
538(0)\end{array}$ & $\begin{array}{l}50(9) \\
647(11)\end{array}$ & 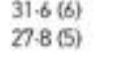 & 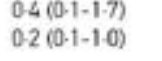 & $\begin{array}{l}0.215 \\
0.056\end{array}$ & 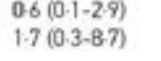 & 0.519 & $\begin{array}{l}7.5(1.36-35) \\
7.0(14-35.99\end{array}$ & $\begin{array}{l}0.609 \\
0.020\end{array}$ \\
\hline NPSI & & & & & & & & & \\
\hline 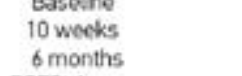 & 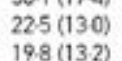 & 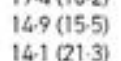 & $\begin{array}{l}180(106) \\
18.51029)\end{array}$ & $\begin{array}{l}50(-77-176) \\
5.5(-699.798)\end{array}$ & $\begin{array}{l}0.437 \\
0.329\end{array}$ & 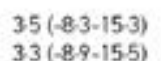 & $\begin{array}{l}0.556 \\
0.526\end{array}$ & 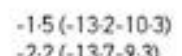 & 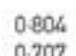 \\
\hline 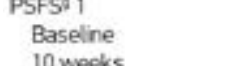 & $7.5(1.9)$ & $66(2.5)$ & 7.1228 & & & & & & \\
\hline 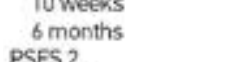 & $2.9(2.3)$ & 25327 & $\begin{array}{l}5.1(36) \\
5.136)\end{array}$ & $0.1(-23-2.5) ?$ & 0.937 & $\begin{array}{l}28(0.10-5.51) \\
28\end{array}$ & 0.023 & 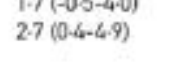 & . \\
\hline 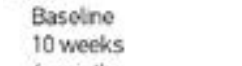 & $\begin{array}{l}6.7(20) \\
3.8(28)\end{array}$ & $\begin{array}{l}56(2.6) \\
32(2.8)\end{array}$ & $\begin{array}{l}62.27) \\
69364\end{array}$ & & & & & & \\
\hline $\begin{array}{l}\text { mimonths } \\
\text { SSFS } 3\end{array}$ & 2.62.7) & $26(35)$ & 45(35) & $08(-20 .-35)$ & 0.575 & $27(100.53)$ & 0.048 & $1.9(-0 .-45)$ & 0.151 \\
\hline $\begin{array}{l}\text { Bassene } \\
10 \text { weeks }\end{array}$ & $\begin{array}{l}7.023) \\
302993 \\
3\end{array}$ & $\begin{array}{l}68(22) \\
232.4)\end{array}$ & $\begin{array}{l}508227 \\
500227\end{array}$ & $-100(-377-18)$ & . & $31(0,-75)$ & 0.012 & $41(146-67)$ & $0: 003$ \\
\hline 6 months & & $14(2.2)$ & & $0.87-36-1]$ & 0.566 & $40(14-65)$ & 0.003 & $67(2.1-73)$ & 0.001 \\
\hline
\end{tabular}

Table 8. (continued)

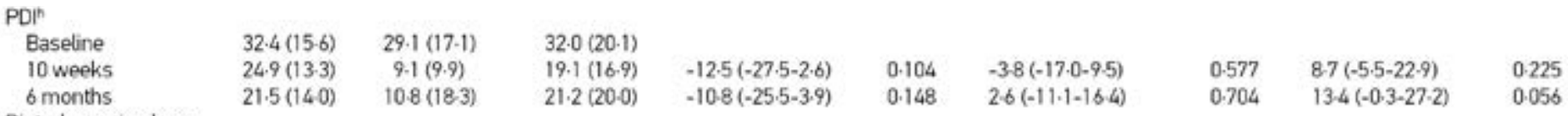

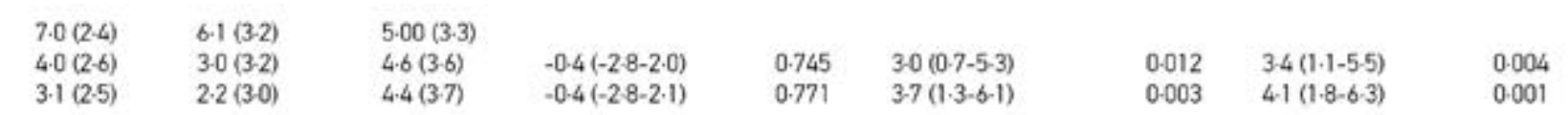

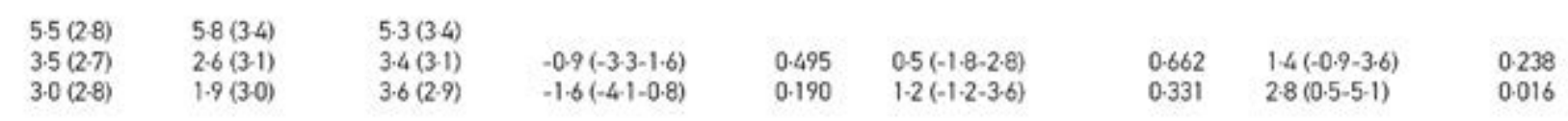

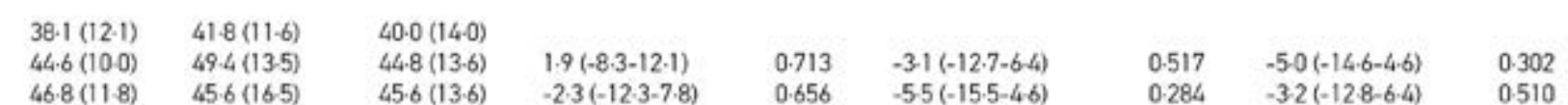

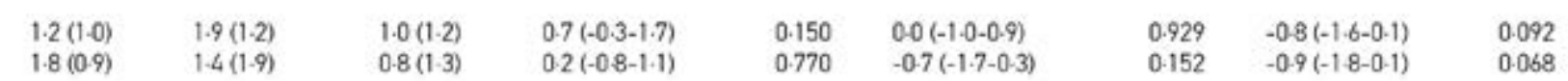

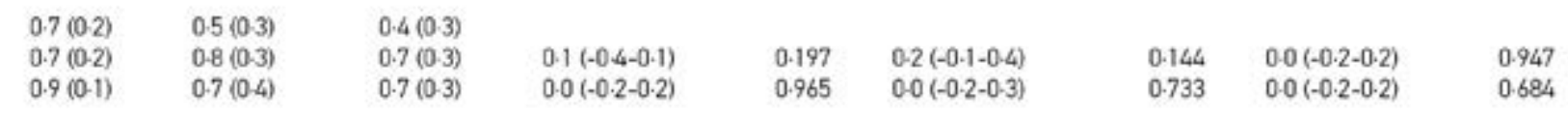

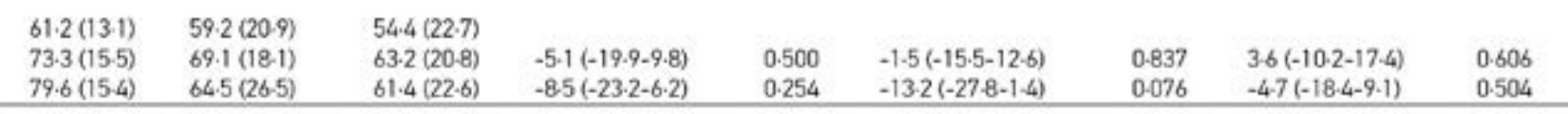
6 months

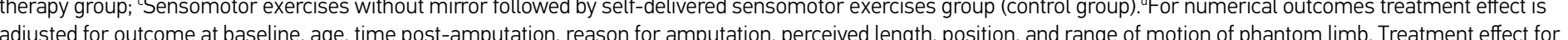

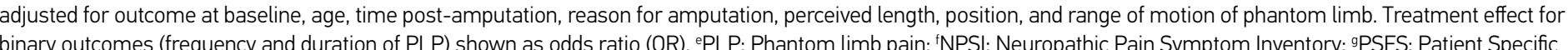

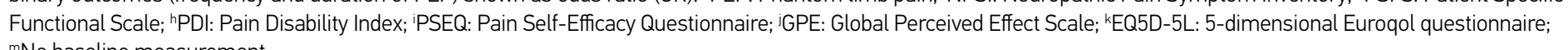




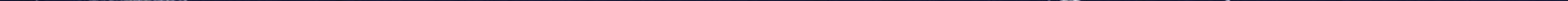




\section{ABSTRACT}

OBJECTIVE: To evaluate the delivery, acceptance and experiences regarding a traditional and teletreatment approach to mirror therapy as delivered in a randomized controlled trial

DESIGN: Mixed method, prospective study.

SETTING: Rehabilitation centres, hospital and private practices.

SUBJECTS: Adult patients with phantom pain following lower limb amputation and their treating physical and occupational therapists.

INTERVENTIONS: All patients received 4weeks of traditional mirror therapy $(n=51)$, followed by 6 weeks of teletreatment $(n=26)$ or 6 weeks of self-delivered mirror therapy $(n=25)$.

MAIN MEASURES: Patient files, therapist logs, log files teletreatment, acceptance questionnaire and interviews with patients and their therapists

RESULTS: In all, 51 patients and 10 therapists participated in the process evaluation. Only 16 patients (31\%) received traditional mirror therapy according to the cli according to he 5 pal Wh sufcient dose. Telearme usage decreased from a therapists Potential bapiers for implementaion of the teltreatment perceived hy patients and therapists were related to insufficient training and support as well as the frequency of technical problems.

CONCLUSION: Traditional mirror therapy and the teletreatment were not delivered as intended in the majority of patients. Implementation of 作 the teletreatment in daily routines was challenging, and more research is needed to evaluate user characteristics that influence adherence and 
INTRODUCTION

Phantom limb pain is a chronic painful sensation following the amputation of a limb that seems to be caused by maladaptive neuroplastic changes in the central and peripheral nervous system. ${ }^{12}$ Up to $80 \%$ of amputees suffer from phantom limb pain ${ }^{34 .}$ that shows no or only

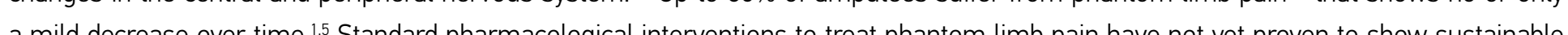
tarcting nemroplastic chas in the (the central nervous system have gained increasing interest during the past years in the treatment ofpatents with phantom limb pain ${ }^{7.8}$ However, the quality of evidence for the effectiveness of these approaches is still low?

Given the limited evidence, a large three-arm multicentre, randomized controlled trial (PAtient Centered Telerehabilitation (PACT) trial) $)^{10.11}$ including a total of 75 lower limb amputees was conducted, in which both a clinical framework for traditional mirror therapy'12 as well as a novel teletreatment using augnented realty mirror therapy ${ }^{\text {Is }}$ were embedded. This randomized controlled trial did demonstrate only small, non-significant effects of the traditional and teletreatment approach to mirror therapy. ${ }^{10}$ One reason for these limited effects may be that treating physical and occupational therapists did not deliver the interventions according to the clinical framework and patients did not use the teletreatment with sufficient dose. The present process evaluation tests this hypothesis and helps to gain more insights on how the interventions were actually used and delivered, and which experiences patients and their treating therapists made. These insights may help to improve the feasibility of the clinical framework for mirror therapy and teletreatments for patients and health care professionals by identifying potential barriers and facilitators for successful implementation.

The following research questions were addressed:

1) Did physical and occupational therapists deliver traditional mirror therapy according to the pre-defined clinical framework?

2) Which digital exercise programs of the novel teletreatment did patients use and to what extent?

3) What were the acceptance rates and experiences of patients and health care professionals regarding

the novel teletreatment?
METHODS

In this prospective process evaluation performed alongside a randomized controlled trial, both quantitative and qualitative methods were used sequentially or concurrently to evaluate the feasibility of two novel interventions. 12.13 The protocol of the randomized controlled triall1 was approved by the ethics committee of the Medical Faculty of Cologne University, Germany (reference no. 13-304) and registered in the ClinicalTrials.gov Register (ID NCT02076490). The main report on the results of the randomized trial was recently published.10

Participants

The process evaluation was conducted at six rehabilitation clinics, one hospital and two private practices in Germany between May 2014 and September 2016. Data were collected firm all controlled trial10 that received at least one session of traditional mirror therapy or the teletreatment respectively. The selection criteria for the trial results. ${ }^{10,1}$

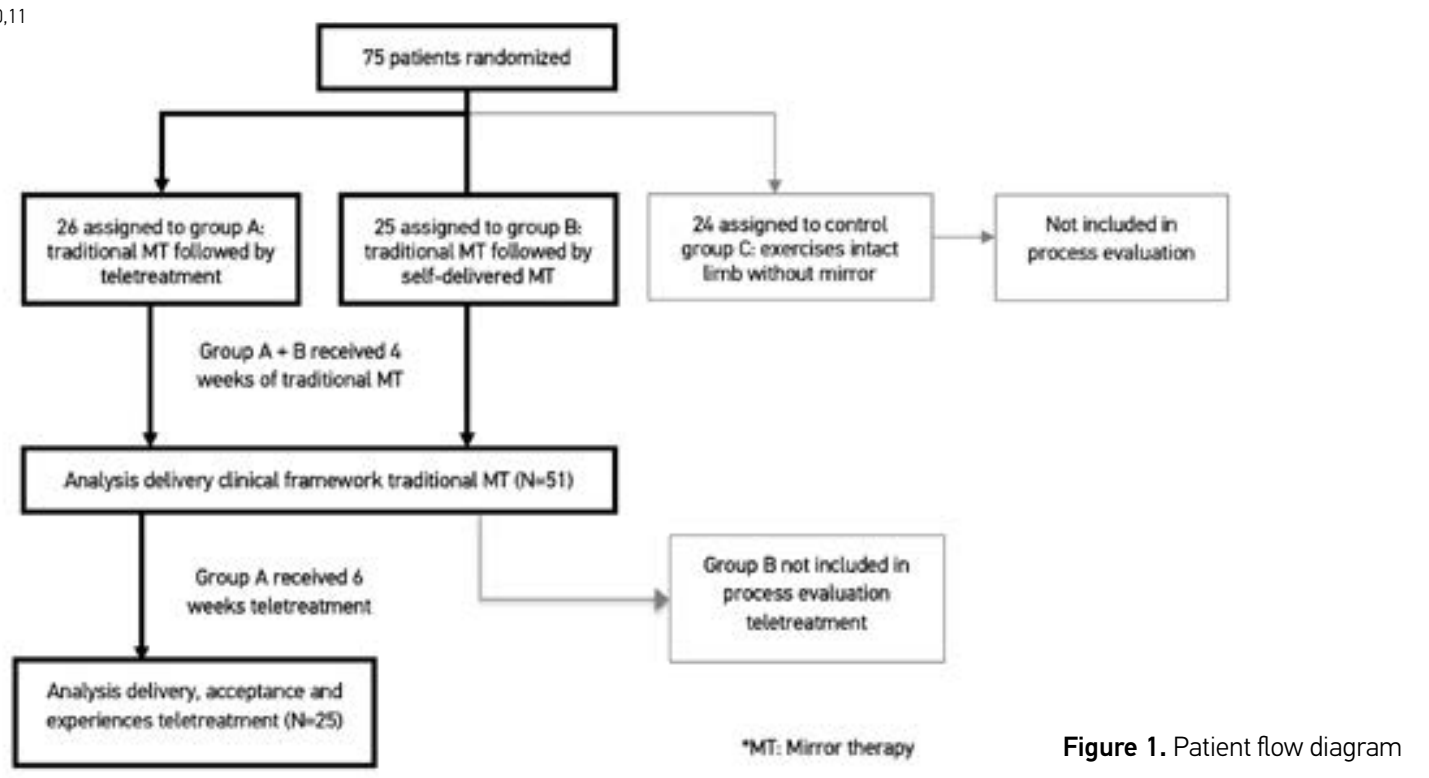


Intervention

Two interventions were evaluated in this process evaluation: traditional mirror therapy and a teletreatment ${ }^{13}$ using augmented reality mirror therapy. Both experimental groups first received traditional mirror therapy according to a clinical framework'12 during the first 4 weeks Thus, both groups were analyzed oge question 1). Regarding the process evaluation of the teletreatment (research questions 2 and 3 ), only patients allocated to the traditional mirror therapy followed by teletreatment group were analysed (Figure 1).

Clinical framework for mirror therapy (weeks 1-4)

The frame and has been described in decal elsewhere. The framework consists of four different mandatory exercise categories: (1) basic motor therapists were instructed to deliver exercises from all mandatory categories during the first sessions and to select those exercises from which the individual patient perceived the most benefl Subsequently, the actual taining phase began and therapists were instructed to also served as home programs for patients to perform self-delivered exercises.

Teletreatment (weeks 5-10)

At the end of the first 4 weeks, therapists had to schedule at least one extra session to instruct patients who were allocated to the teletreatment group on how to use the teletreatment, which was subsequently used by patients for b weeks at home. The main functionalities of the

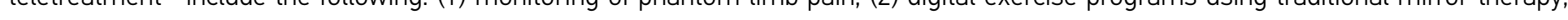
(3) augmented realty mirror therapy using the tablet-integrated camera, (4) audio-visual instruction of mental practice, (5) limb laterality recognition training, (6) comm nuication with he personal herapist and other patients and ( $)$ background information on different topics (e.g. to use the teletreatment as often as his wish but witho Un futher supor of the treating therapist

All therapists received a half-day standardized training by the principal investigator about the theoretical background of the intervention, how to implement the mirror therapy framework and how to use the teletreatment. The therapists received additional written information about mirror therapy (e.g. course map including the framework), materials to facilitate self-delivered mirror therapy (e.g. patient logs and leafle) and the teletreatment (e.g. user manual). During the intervention period, the principal investigator regularly called therapists to discuss potential problems regarding the implementation of the clinical framework and the use of the teletreatment.

Data collection

Different qualitative and quantitative data collection methods were used to obtain information on the desired process measures as shown in Table 1.

Table 1. Overview of different measures used for process evaluation

\begin{tabular}{|c|c|c|c|c|c|}
\hline Measure & Process variable & $\begin{array}{l}\text { Timing of } \\
\text { measure }\end{array}$ & Completed & $\begin{array}{l}\text { Response } \\
\text { rate }\end{array}$ & Comments \\
\hline $\begin{array}{l}\text { Patient files } \\
\text { (N treated: } 51 \text { ) }\end{array}$ & $\begin{array}{l}\text { Dose delivered } \\
\text { traditional MT }\end{array}$ & Weeks $1-4$ & $\mathrm{~N}=51$ & $100 \%$ & \\
\hline $\begin{array}{l}\text { Therapist logs } \\
\text { (N handed: 51) }\end{array}$ & $\begin{array}{l}\text { Delivery MT } \\
\text { framework }\end{array}$ & Weeks $1-4$ & $\mathrm{~N}=38$ & $75 \%$ & $\begin{array}{l}13 \text { logs missing } \\
\text { (not reported) }\end{array}$ \\
\hline $\begin{array}{l}\text { Log files } \\
\text { (N instructed: 25) }\end{array}$ & $\begin{array}{l}\text { Use of } \\
\text { teletreatment }\end{array}$ & Weeks 5-10 & $\mathrm{N}=25$ & $100 \%$ & \\
\hline $\begin{array}{l}\text { Acceptance questionnaire } \\
\text { patients } \\
\text { (N handed: 25) }\end{array}$ & $\begin{array}{l}\text { Acceptance } \\
\text { teletreatment }\end{array}$ & At 10 weeks & $\mathrm{N}=23$ & $92 \%$ & $\begin{array}{l}2 \text { patients dropped- } \\
\text { out and were } \\
\text { unavailable for } \\
\text { measurement }\end{array}$ \\
\hline $\begin{array}{l}\text { Interviews patients } \\
\text { (Ninvited: 25) }\end{array}$ & $\begin{array}{l}\text { Experiences } \\
\text { teletreatment }\end{array}$ & At 10 weeks & $\mathrm{N}=23$ & $92 \%$ & $\begin{array}{l}2 \text { patients dropped- } \\
\text { out and were } \\
\text { unavavalable for } \\
\text { measurement }\end{array}$ \\
\hline $\begin{array}{l}\text { Acceptance questionnaire } \\
\text { therapists } \\
\text { (Nhanded: } 10)\end{array}$ & $\begin{array}{l}\text { Acceptance } \\
\text { teletreatment }\end{array}$ & $\begin{array}{l}\text { End of } \\
\text { randomized } \\
\text { trial }\end{array}$ & $\mathrm{N}=10$ & $100 \%$ & \\
\hline $\begin{array}{l}\text { Interviews therapists } \\
\text { (N invited: 10) }\end{array}$ & $\begin{array}{l}\text { Experiences } \\
\text { teletreatment }\end{array}$ & $\begin{array}{l}\text { End of } \\
\text { randomized } \\
\text { trial }\end{array}$ & $\mathrm{N}=10$ & $100 \%$ & \\
\hline
\end{tabular}



Demographic characteristics of patients such as date, reason and level of amputation were assessed through a self-assessment
questionnaire before the start of the intervention. Background characteristics of therapists (e.g. age, profession, number of patients treated) were recorded in the first section of the acceptance questionnaire (see Supplemental Appendix).

Regarding the delivery of he clinical framework during the firs 4 weeks (research question 1 ), the number of individual sessions that took place was assessed by extracting dala from indivdual palient hes and he herapis logs. The log we adherence with the predefined clinical framework. In the log, the frequency and duration of individual sessions per week, type of exercises, 作 of sessions they delivered to introduce patients to the teletreatment at the end of the firs 4 weeks,

Regarding patients' use of the teletreatment (research question 2), the frequency, duration and type of teletreatment component used were af he vistrich

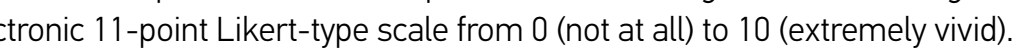

With respect to the acceptance rates and user experiences of the teletreatment (research question 3), a self-administered acceptance questionnaire and an individual phone interview between each individual user and the principal investigator took place. The self-developed patient and therapist questionnaire consisted of nine items based on different categories related to the technology acceptance model 14,15 (see Supplemental Appendix). Each item was scored on an 11-point Likert-type scale from 0 (totally disagree) to 10 (totally agree). In addition, two open questions regarding the overall opinion on the teletreatment were provided. These open questions served as starting point for the individual phone interview in which the experiences of the users regarding the teletreatment as well as positive and negative aspects were assessed. The principal investigator took notes and collected individual quotes of the users.

Data analysis

The quantitative data from the pre-structured patients' files and therapists' logs were extracted by a research assistant and were then summanized in an excel spreadsh traditios mis cor therapy to be delived according to the clinical fram used.
Regarding the use of the teletreatment, the software developer (Kaasa health, Germany) sent all log files of individual patients that were automatically registered by the teletreatment to the principal investigator (A.R.) in an excel file. All individual log files were then filtered for the corresponding intervention period of weeks $5-10$ and 6 months follow-up (weeks $11-24$ ) by the principal investigator. Patients who used at least 10 teletreatments with a minimal duration of 5 minutes during the 6 weeks of intervention period were considered as compliant with the protocol.

All quantitative data were descriptively analysed, and the sum scores for the individual items of the acceptance questionnaires were visually displayed using frequency tables and bar graphs.

All qualitative data from open questions discussed during the phone interviews with patients and therapists were summarized for every Subsequently, the summary was sent to the interview approved summary.

\section{RESULTS}

Regarding the delivery of the clinical framework for mirror therapy during the first 4 weeks, a total of 51 patients with a mean (SD) age of 61.1 (13.9) years took part in the 1). Twenty-five out of these 51 patients received the intended introduction to the teletreatment and were involved in the process evaluation regarding the use, acceptance and experiences of the teletreatment. In addition, six physical and four occupational therapists with a mean (SD) age of $43.3(11.0)$ years (Table 4), who delivered traditional mirror therapy as well as the teletreatment, participated in the process evaluation. Table 1 presents the response rates for the different measures used for process evaluation.

Regarding the type of exercises delivered, basic motor exercises were used in all patients, sensory exercises as well as motor exercises

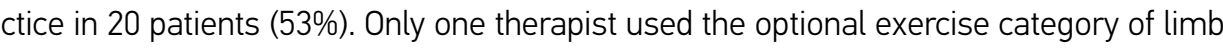
laterality recognition training in one patient. Therapists reported adverse events in 10 patients $(26 \%)$. Details about these events are provided elsewhere 10 
Delivery of clinical framework for mirror therapy (research question 1)

During the first 4 weeks, thirtyseven patients $(73 \%)$ received the mandatory therapy amount of at least 10 sessions. The number of individual mirror therapy sessions ranged from 1 to 20 , with an average of 9.8 (SD 2.7) sessions. However, according to the therapist logs $(n=38)$, only 16 patients $(31 \%)$ received traditional mirror therapy according to the clinical framework as they had exercises from all mandatory categories of the framework as well as the mandatory treatment dose of at least 10 sessions.

Table 2. Baseline characteristics of patients participating in the process evaluation

\begin{tabular}{|c|c|c|}
\hline Variable & $\begin{array}{l}\text { Group A: } \\
\text { Teletreatment } \\
(n=26)\end{array}$ & $\begin{array}{l}\text { Group } A+B: \\
\text { Traditional MT } \\
(n=51)\end{array}$ \\
\hline Age (mean, SD) & $59.7(16.1)$ & $61 \cdot 1(13-9)$ \\
\hline Gender, male & $80.8(21)$ & 68.6(35) \\
\hline Time post amputation, months, (median, IRR) & $56 \cdot 5(24.5-226-3)$ & $38-0(25-219)$ \\
\hline $\begin{array}{l}\text { Mean intensity PLP previous week (mean, SD) } \\
\text { Side of amputation, right }\end{array}$ & $\begin{array}{l}5.9(1.9) \\
69.2(18)\end{array}$ & $50.9272)$ \\
\hline \multicolumn{3}{|l|}{ Reason for amputation } \\
\hline Trauma & $38.5(10)$ & 35.3(18) \\
\hline Diabetes & & \\
\hline Dysvascular & $23: 1(6)$ & 23.5 (12) \\
\hline Tumor & $15.4(4)$ & 17.6 (9) \\
\hline Other (eg, infection) & & \\
\hline
\end{tabular}

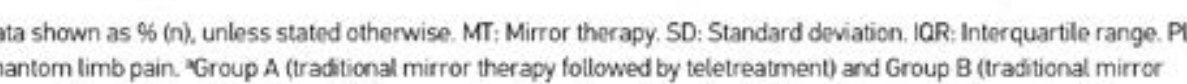
herapy followed by self-didivered MT) were andilysed together during the first 4 weeks as patents received the same
Usage of the teletreatment (research question 2)

In 18 out of 25 patients $(72 \%)$ who received the introduction to the teletreatment, one session was used to introduce them to the teletreatmen with the duration of sessions varying between 5 and 30 minutes. In six patients $(24 \%)$ the session was not given additionally but was incorporated in one of the 10 mandatory mirror therapy sessions delivered auring the first 4 weeks.

Durng the bweeks of telerealment intervention period (weeks $5-10), 22$ oun of he 25 palients (88\%) used the only 14 patients (56\%) used it with sufficient dose according to the predefined protocol. The majority of patients $(n=19 ; 76 \%)$ performed augmented reality miritor herapy, and 15 palients (60\%) used the digital exercise programme of tradilional miror herapy as well as limb laterality recognition training. Patients performed a total median number of 31 (interquarthe range $($ IQR $)=12-50$ ) sessions with a total median

Between weeks 11 and 24 (follow-up at 6 months), the frequency and duration of teletreatment usage decreased, with 17 patients (68\%) used the digital exrcis teletreatment sessions in this time period deceased to $19(\mathrm{IQR}=9-104)$ and the median usage time to 361 minutes $(1 \mathrm{OR}=48-1091)$ Three of 1259 minutes (IQR $=1162-1445.5$.

Acceptance rates of patients regarding the teletreatment (research question 3)

Overall, patients showed moderate to high agreement related to the different aspects of the acceptance auestionnaire ranging from average scores of 6.1 (SD 3.7) to 9.3 (SD 1.3) on the 11-point Likert-type scale (Table 4, Supplemental Figure S1). the highest with average scores ranging from 8.8 (SD 1.7) to 9.3 (SD 1.3).

Technical problems appeared relatively frequent and it was not always possible to fix bugs immediately, which negatively affected the usability of the teletreatmen 
Table 3. Use of teletreatment components at 10 weeks and 6 months follow-up

\begin{tabular}{lccc}
\hline & Weeks 5-10 & & Weeks 11-24 \\
Traditional mirror therapy (N patients) & 15 & 10 \\
\hline Traditional mirror therapy (min) & $253(37-592.5)$ & & $692(126-1344.3)$ \\
Traditional mirror therapy vividness* & $3.5(2.4-6.2)$ & & $5.0(4.2-6.5)$ \\
Augmented reality mirror therapy (N patients) & 19 & 11 \\
\hline Augmented reality mirrot theray (min) & $57(22-125)$ & $51(26-362.5)$ \\
Augmented reality mirror therapy vividness* & $5.0(2.2-6.2)$ & $5.0(3.9-7.8)$ \\
Mental practice (N patients) & 9 & 3 \\
\hline Mental practice (min) & $19(6-188)$ & $1259(1162-1445.5)$ \\
Mental practice vividness* & $2.1(1.5-3.3)$ & $8.4(7.2-8.5)$ \\
Laterality recognition training (N patients) & 15 & 10 \\
\hline Laterality recognition training (min) & $30(13.5-76)$ & $35.5(14.5-166.5)$ \\
Relaxation training (N patients) & 5 & 1 \\
\hline Relaxation training (N sessions) & $2(1-5)$ & 78 \\
\hline Number online sessions & $31(12-50)$ & $19(9-104)$ \\
\hline Usage time (min) & $198(86.5-527)$ & $361(48-1091)$ \\
\hline
\end{tabular}

Number online sessions

$3198(86.5-527) \quad 361(48-109)$

Data shown as Median (Interquad
(0=not at all; $10=$ extremely vivid).
Acceptance rates of therapists regarding the teletreatment (research question 3)

Overall, therapists showed slightly lower acceptance rates compared to patients but the same trends were observed regarding the different items of the acceptance questionnaire (Table 4, Supplemental Figure S2). Again, the perceived behavioral control to use the system and items related to the perceived ease of use of the system were rated higher with average scores ranging from 7.1 (SD 1.7) to 8.4 (SD 1.6). Lowe average scores of 4.8 (SD 2.4) and 5.8 (SD 2.2) were found for the perceived usefulness and efficacy of the teletreatment for the daily work of therapists (e.g. delivery and monitoring of the intervention).

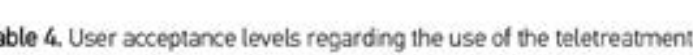

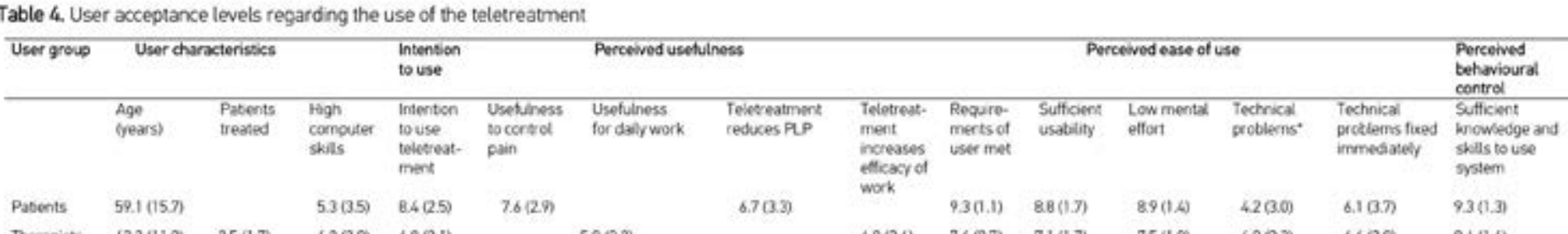

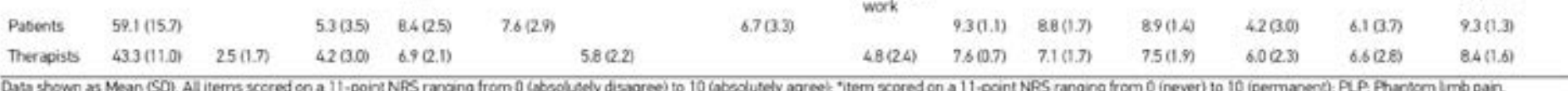

Experiences of patients regarding the teletreatment (research question 3)

SIx main themes emerged from the patient inter-Views regarding their experiences related to the teletreatment as shown in Supplementa (4) aspects related to digital exercise programmes, (5) technical problems and difficulties handling the tablet and (6) instruction, personal contact and feedback

Perceived benefits that were mentioned by patients were related to different domains such as phantom pain, sense of control or body image: In case of acute pain attacks, it acts like a strong drug and immediately reduces 
Patients appreciated the mobility of the teletreatment and that exercises could be performed independently of time and place, which facilitated integration in their daily routines.

I used the tablet on business trips to China in the airplane or in the hotel. (Male, 44 yrs).

The majority of patients experienced technical problems when using the teletreatment. In the beginning of the trial, the mobile application delayed data transfer Regarding the theme 'instruction personal contact and feedback', two patients mentioned that they were insufficiently introduced to the teletreatment by their therapist and one patient needed additional support by a family member in order to feel more confident in using the technology.

The therapist came a long for 5 minutes and gave me the tablet without further
explanation and I wasn't technologicallyskilled, sol didn't use it at home (Male, 77 yrs)

Various suggestions for improvement of the teletreatment were made by patients referring to four different categories: (1) more variation in exercises, (2) personalize instructions, (3) messaging and (4) operation system (Supplemental Table S2).

Experiences of therapists regarding the teletreatment (research question 3)

Table S2: (1) perceived benefits, (2) creating a long-term relationship with patients, (3) aspects related to digital exercise programmes, (4) design and usability, (5) technical problems, (6) training of the users and (7) selection of eligible patients.

Regarding the main theme 'perceived benefits', most of the therapists appreciated the practicability and mobility of the teletreatment, which enabled them to work more independently regarding the space and location needed to deliver the intervention. Interestingly, therapists also perceived the use of the teletreatment as a sign of quality and innovation of their own work by using information and communication technology for rehabilitation purposes:

My portfolio and skills improved and you are better off towards the patient (Female, 57 yrs)
The majority of therapists suffered from similar technical problems that were also described by most of the patients related to bugs during

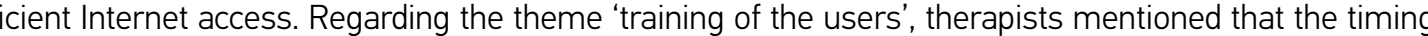
and frequency of training was not adequate to facilitate their routine in using the teletreatment.

Now we were trained before the trial started, but the first patient started only 8 weeks later; with this few amount of patients

According to therapists it is important to carefully select eligible patients beforehand, as they assumed that e.g., a certain degree of computer (1) enhance exercise programs, (2) peer support and (3) incorporate online community moderator (Supplemental Table S2). 
DISCUSSION

This process evaluation showed that in the majority of patients $(n=35,69 \%)$, traditional mirror therapy was not delivered according to the clinical framework. Furthermore, nearly hall of patients did not use the teletreatment with the minimal mandatory treatment dose according to the predefined protocol $(n=11,44 \%)$. The digital exercise programs of traditional and augmented reality mirror therapy were used most often. Likert-type scale. Therapists showed slightly lower acceptance rates ranging on average from 4.8 to 8.4 regarding the individual items of the

Analysis of user experiences showed that the majority of patients who did use the teletreatment mentioned potential benefits from delivering the intervention and intended to use it after the trial. Patients and therapists agreed on the importance of sufficient training and support of the users as well as the absence of technical problems, which were regarded as potential facilitators for implementation. a small number of

per for minor herapy we docided to suply therapists with suffcient information to guide them through the clinical process from patient intake to discharge, but at the same time enable them to tailor the intervention to the preferences of the individual patient. As a consequence, theranists particularly delivered less mental practice and limb laterality recognition training, since they also did not use them prior to the trial. This might suggest that some therapists were unable to sufficiently embed the protocol into their professional routines.

The low adherence rates observed regarding the teletreatment might be related to limited skills and experiences of patients and therapists on 政 being unfamiliar with the technology. Probably, more time was needed to gain experience with the teletreatment as well as more intensive training and supervision during the randomized controlled trial. It has been shown that insufficient training of therapists can be an important structured and intense training of patients would probably have been useful too. A recent study $y^{17}$ showed that patients regarded sufficient te

In the PACT trial we decided to investigate the effects of traditional mirror therapy during the first 4 weeks as evidence so far was weak and not to introduce patients allocated to the teletreatment group before the last week to the technology.

Therefore, the second reason for low adherence rates might be that some patients already perceived sufficient pain reduction during the first 4 weeks of traditional mirror therapy and thus, might have had no necessity to further use the teletreatment during the subsequent study period.

In this process evaluation, therapists perceived less benefits for their own work by using the teletreatment. This might suggest that the teletreatment did not succeed in making the work for therapists easier, which seems to be a key factor to clinicians' acceptance of eHealth. ${ }^{18}$

Strengths and limitations of the study

A strength of this process evaluation is that within the PACT trial participants from different centres from primary and secondary care such rehabilitation practice in Germany has been included Furhectude, Also, the outcomes of the PACT trial were not known at the time of data collection for this process evaluation and thereby could not have

biased the outcomes.
As mentioned before, a weakness of this study is that most therapists only treated a few patients leading to a lack in gaining routine in using
the teletreatment This might have influenced the outcomes of the acceptance questionnaire and interviews Overall therapists seemed to be the teletreatment. This might have influenced the outcomes of the acceptance questionnaire and interviews. Overall, therapists seemed to be In addition, patients and therapists who took part in the trial and process evaluation might have had a more positive attitude towards the teletreatment than non-responders.

Results compared to other studies

This study is the first process evaluation on non-pharmacological interventions such as mirror therapy and a teletreatment using augmented reality mirror therapy perforet alongside a randomized controlled trial in patients with phantom limb pain. The published protocols for mintror ther 19 with a sparse description of intervention characteristics represent a more rigid programme mainly focussing on basic motor health care professionals were trained and how the implementation of the intervention was monitored Some studies evaluated patient adherence with a training diary20.21 or weekly phone calls. ${ }^{21}$ All published treatment protocols seemed to be feasible, but data on different process measures is sparse.

Another process evaluation on the feasibility of a clinical framework for mental practice in stroke patients ${ }^{22}$ showed that applying the

framework in clinical practice was harder than expected and posed many challenges.
Regarding teletreatments for patients with phantom limb pain, we are aware of only one other study that has been published ${ }^{23}$ in which two 
patients following lower limb amputation received instructions how to self-deliver mirror therapy and how to self-report pain assessments by e-mail. The intervention was feasible, but no data were published regarding compliance, user acceptance and experiences related to the teletreatment.

Implications for research and clinical practice

ment of the intervention including an evidence-based and user-centred approach ${ }^{22,13}$ does not in particular technolog-driveptance, adherence and hence effects. The implementation of novel complex interventions in clinical practice, characteristics and skills influence their adoption. 1,2 Thus , for successful im of face-to-face and online therapy needs to be tailored to the needs, preferences and characteristics of individual patients and therapists. ${ }^{24}$ Therapists might consider offering patients with limited technical and Internet skills or increased physical and cognitive impairments more use of the intervention needs to be personalized regarding dose and timing to provide the necessary information when it is actually needed Future research should identify the appropriate proportion between online and face-to-face sessions for different groups of patients in order to develop personalized blended care interventions. ${ }^{52526}$ More research is needed to evaluate user characteristics that influence teletreatment adherence, which patients benefit most from blended care and how technology features can be optimized to develop tailored implementation strategies.

Clinical Messages
- Traditional mirror therapy was not delivered according to the clinic
- Mramework in the majority of patients.
dose ofter 4 wetients did not use the teletreatment with sufficient
- Patients showed mirror therapy.
specific benefits from using the teletreatment than the therapists
reported.

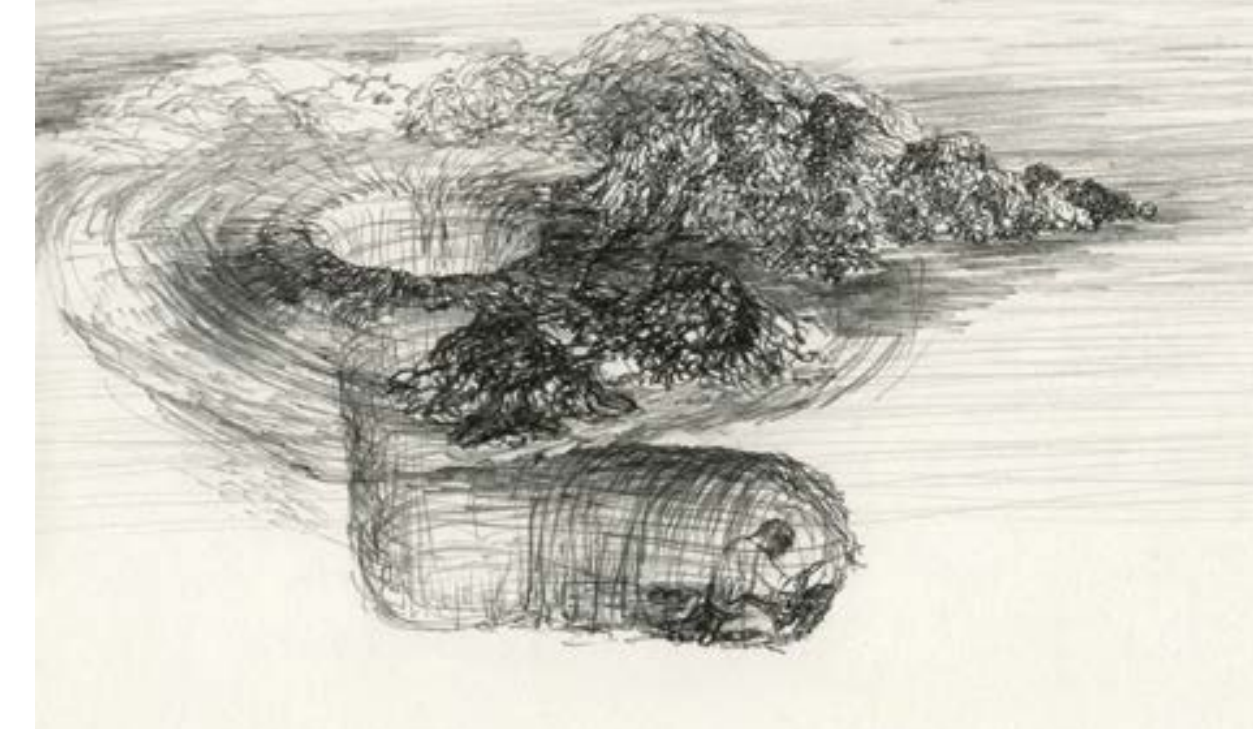




\section{REFERENCES}

Flor H. Phantom-limb pain: characteristics, causes, and treatment. Lancet Neurol 2002; 1(3): 182-189. Makin TR, Scholz J, Filippini N, et al. Phantom pain is associated with preserved structure and function in the former hand area.

Nat Commun 2013; 4: 1570 .
3. Ephraim PL, Wegener ST, Mackenzie EJ, et al. Phantom pain, residual limb pain, and back pain in amputees: results of a national survey. Arch Phys Med Rehabil 2005; 86(10): 1910-1919.

列 and phantom pain.

Alviar MJ. Hale T and Dungca M. Pharmacologic interventions for treating phantom limb pain. Cochrane Database Syst Rev 2016 10: CD006380.

Foell J, Bekrater-Bodmann R, Diers M, et al. Mirror therapy for phantom limb pain: brain changes and the role of body representation. Eur J Pain 2014; 18(5): 729-739.

8. Maclver K, Lloyd DM, Kelly S, et al. Phantom limb pain, cortical reorganization and the therapeutic effect of mental imagery. Brain 2008: 131(P+8): 2181-2191.

Herrador Colmenero Le Perez Marmol JM Marti-Garcia C etal Efectiveness of miror therapy, motorimagery, and virtual feedhack on phantom limb pain following amputation a systematic review, Prosthet Orthot Int 2018: 42(3): 288-298.

10. Rothgangel A, Braun S, Winkens B, et al. Traditional and augmented reality mirror therapy for patients with chronic phantom limb pain (PACT study): results of a three-group, multicentre single-blind randomized controlled trial. Clin Rehabil 2018; 32(12):

$1591-1608$.

11. Rothgangel AS, Braun S, Schulz RJ, et al. The PACT trial: PAtient Centered Telerehabilitation: effectiveness of software-supported and traditional mirror therapy in patients with phantom limb pain following lower limb amputation: protocol of a multicentre randomised controlled trial. J Physiother 2015; 61(1): 42; discussion 42.

12. Rothgangel $\mathrm{A}$, Braun $\mathrm{S}$, de Witte $\mathrm{L}$, et al. Development of a clinical framework for mirror therapy in patients with phantom limb pain: an evidence-based practice approach. Pain Pract 2016; 16(4): 422-434.
3. Rothgangel A, Braun S, Smeets R, et al. Design and development of a telerehabilitation platform for patients with phantom limb pain: a user-centered approach. JMIR Rehabil Assist Technol 2017; 4(1): e2.

Davis FD. Perceived usefulness, perceived ease of use, and user acceptance of information technology. MIS Q 1989; 13: 319-340. Davis $\mathrm{FD}$, Bagozz RP and Warshaw PR. User acceptance of computer technology: a comparison of two theoretical models. Manage Sci 1989; 35: 982-1003.

16. Cameron J, Naglie G, Silver FL, et al. Stroke family caregivers' support needs change across the care continuum: a qualitative study using the timing it right framework. Disabil Rehabil 2013; 35(4):315-324

17. de Vries $\mathrm{HJ}, \mathrm{Kloek} \mathrm{CJ}$, de Bakker $\mathrm{DH}$, el al. Determinants of adherence to the online component of a blended intervention for patients with hip and/or knee osteoarthritis: a mixed methods study embedded in the e-exercise trial. Telemed J E Health 2017 23(12): 1002-1010.

18. Wells $S$, Rozenblum $R$, Park A, et al. Organizational strategies for promoting patient and provider uptake of personal health records. $J$ Am Med Inform Assoc 2015; 22(1): 213-222.

19. Barbin J, Seetha V, Casillas JM, et al. The effects of mirror therapy on pain and motor control of phantom limb in amputees: a systematic review. Ann Phys Rehabil Med 2016; 59(4): 270-275.

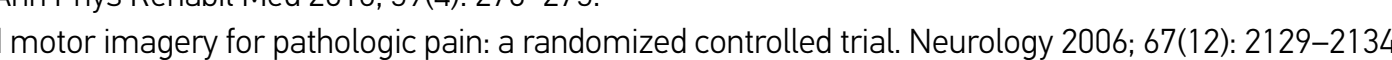

21. Darnall BD and Li H. Home-based self-dellvered mirror therapy for phantom pain: a pilot study. J Rehabil Med 2012; 44(3): 254-260. 22. Braun $\mathrm{SM}$, van Haastregt $\mathrm{JC}$, Beurskens $\mathrm{AJ}$, et al. Feasibility of a mental practice intervention in stroke patients in nursing homes

23. a process evaluation. BMCNeurol 2010; 10:74.

作

24. Wentzel J, van der Vaart R, Bohlmeijer ET, et al. Mixing online and face-to-face therapy: how to benefit from blended care in mental 25.

Erbe $\mathrm{D}$, Eichert $\mathrm{HC}$, Riper $\mathrm{H}$, et al. Blending face-to-face and internet-based interventions for the treatment of mental disorders in . 
APPENDIX CHAPTER 7

SUPPLEMENTARY MATERIAL 
APPENDIX 1. Patient acceptance questionnaire telerehabilitation

Below you will find questions regarding your personal background and several statements regarding the use of the telerehabilitation. Please provide a score for each statement in how far you agree or disagree with the statement given.

A. Your personal background

1) What is your age?

I am years old.

2) Your sex

$\square$ female

$\square$ male

3) I am very skilled in using computers

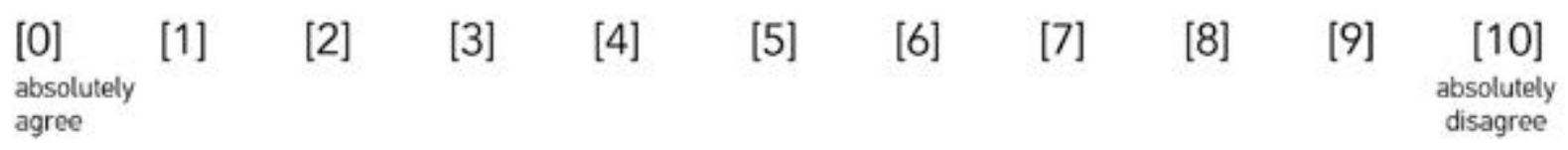

\section{B. Intention to use the telerehabilitation}

4) Assuming that I have access to the telerehabilitation, I intend to use it.

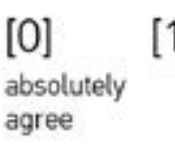
[3] [4]
[5]
6] [7] [8]
[9] $\begin{gathered}{[10]} \\ \text { absolutely }\end{gathered}$

C. Perceived usefulness of the telerehabilitation

5) The telerehabilitation is useful to control my pain.
[0]
absolutely
agree
[2] [3]
[3] [4]
[5]
[6] [7]
[8]
[9] [10]

6) The use of the telerehabilitation reduces my phantom limb pain.
[0]
absolutely
agree
[2]
[3] [4]
[5]
[6] [7]
[7] [8]
[9] [10]

7) The content and functionalities of the telerehabilitation meet my requirements.
[0]
[2]
$[3] \quad[4]$
[5] [6]
[7] [8
[9] [10] 
8) I think the telerehabilitation is easy to use

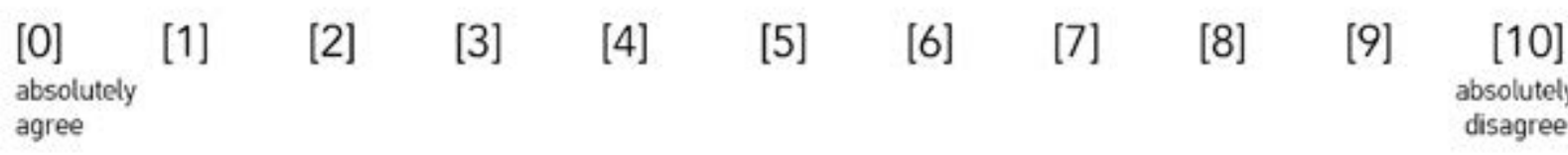

9) The mental effort to use the telerehabilitation is low.

$\left[\begin{array}{llllllllll}{[0]} & {[1]} & {[2]} & {[3]} & {[4]} & {[5]} & {[6]} & {[7]} & {[8]} & {[9]}\end{array}\right]$

10) How often did technical problems during the use of the telerehabilitation occur?

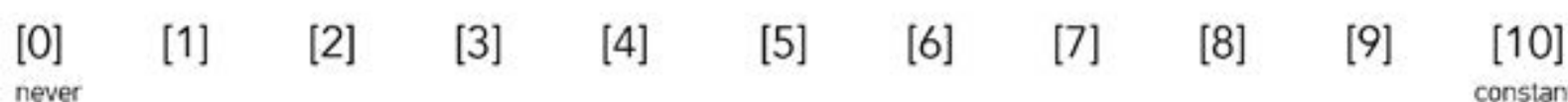

11) The technical problems were fixed immediately.
[0] [1] [2] [3]
$[4]$
$[5] \quad[6]$
[7] [8]
[9] [10]
absolutely
agree
(1)

E. Perceived use-oriented self-efficacy

12) I think that I have sufficient knowledge and skills to use the telerehabilitation.

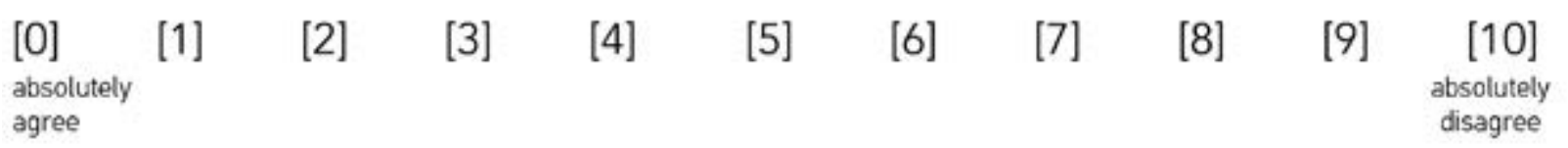

F. Additional comments regarding the use of the telerehabilitation

What were positive aspects of using the telerehabilitation?

\section{What were negative aspects of using the telerehabilitation?}


APPENDIX 2. Therapist acceptance questionnaire telerehabilitation

Below you will find questions regarding your personal background and several statements regarding the use of the telerehabilitation. Please provide a score for each statement in how far you agree or disagree with the statement given.

\section{A. Personal background}

1) What is your age?

Iam years old.

2) What is your profession?

$\square$ Occupational therapist

$\square$ Physical therapist

3) How many patients have you treated using the telerehabilitation so far?

Number of patients:

4) I am very skilled in using computers
$[0][1]$
[2] [3]
[4]
[5] [6]
9] $\quad[10]$

\section{B. Intention to use the telerehabilitation}

5) Assuming that I have access to the telerehabilitation, I intend to use it for patient care.
[0] [1]
[2] [3]
$[4$
[5] [6]
[7] [8]
[9] [10]

C. Perceived usefulness of the telerehabilitation

6) I think the telerehabilitation is useful for the care of my patients.
[0]
absolutely
agree
2] [3] [4]
[5]
[6] [7]
[8] [9] [10]

7) The use of the telerehabilitation enhances the effectiveness of my treatment.
[0]
absolutely
agree
[2] [3]
$[4$
[5] [6]
[7]
[9] $\quad[10]$

8) The content and functionalities of the telerehabilitation meet my requirements.
$\left.\begin{array}{l}{[0]} \\ \text { absolutely }\end{array}\right]$
[2] [3]
$[4]$
[5] [6]
[7] [8]
[9] $\underset{\text { absolutely }}{[10]}$ 
D. Perceived ease of use

9) I think the telerehabilitation is easy to use.

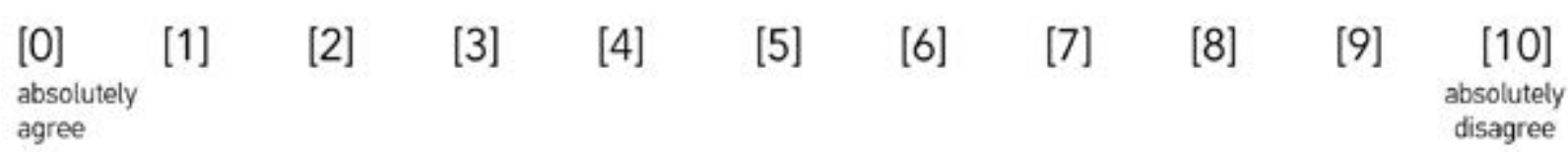

10) The mental effort to use the telerehabilitation is low.
$[0] \quad[1] \quad[2] \quad[3]$
$[4]$
$[5]$
$[7] \quad[8]$
[9] [10
$\begin{array}{lll}\substack{\text { absolutely } \\ \text { agree }} & \begin{array}{c}\text { absolutely } \\ \text { disagree }\end{array} \\ & \end{array}$

11) How often did technical problems during the use of the telerehabilitation occur?

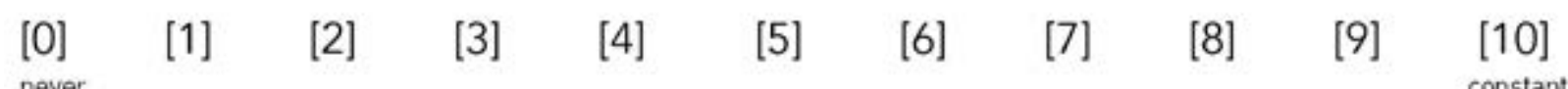

12) The technical problems were fixed immediately.
[7] [8]
[9] [10]
absolutely
agree

E. Perceived use-oriented self-efficacy

13) I think that I have sufficient knowledge and skills to use the telerehabilitation

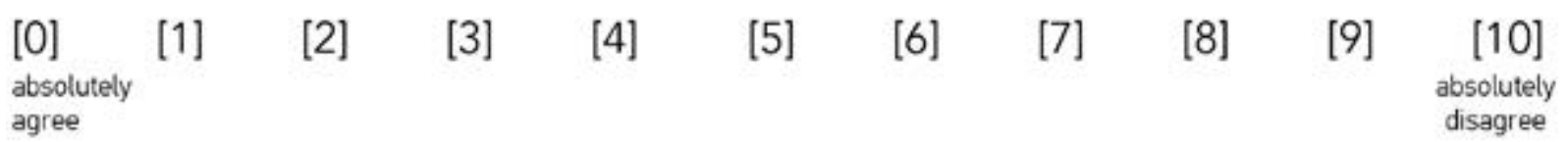

F. Additional comments regarding the use of the telerehabilitation

What were positive aspects of using the telerehabilitation?

\section{What were negative aspects of using the telerehabilitation?}




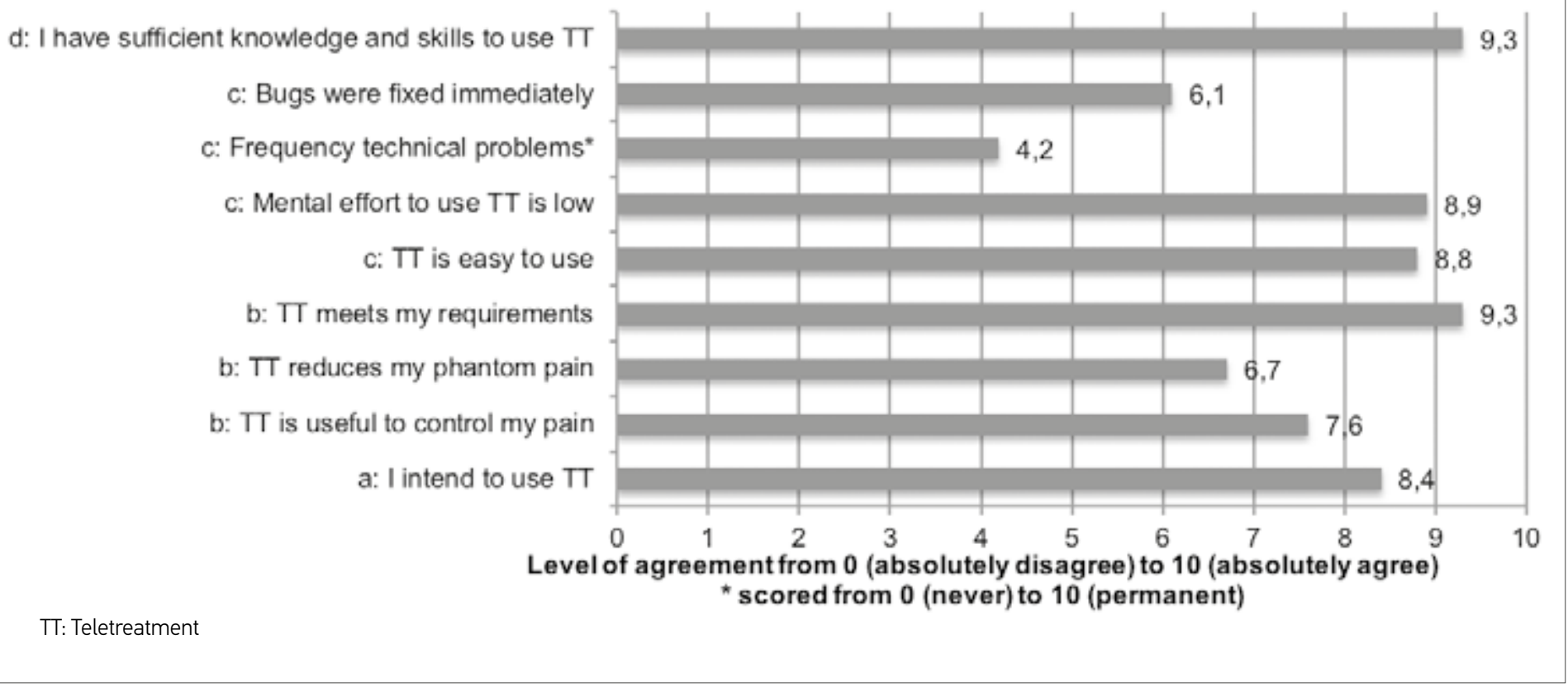

Supplemental Figure 1. Sum scores from different items of the patient acceptance questionnaire

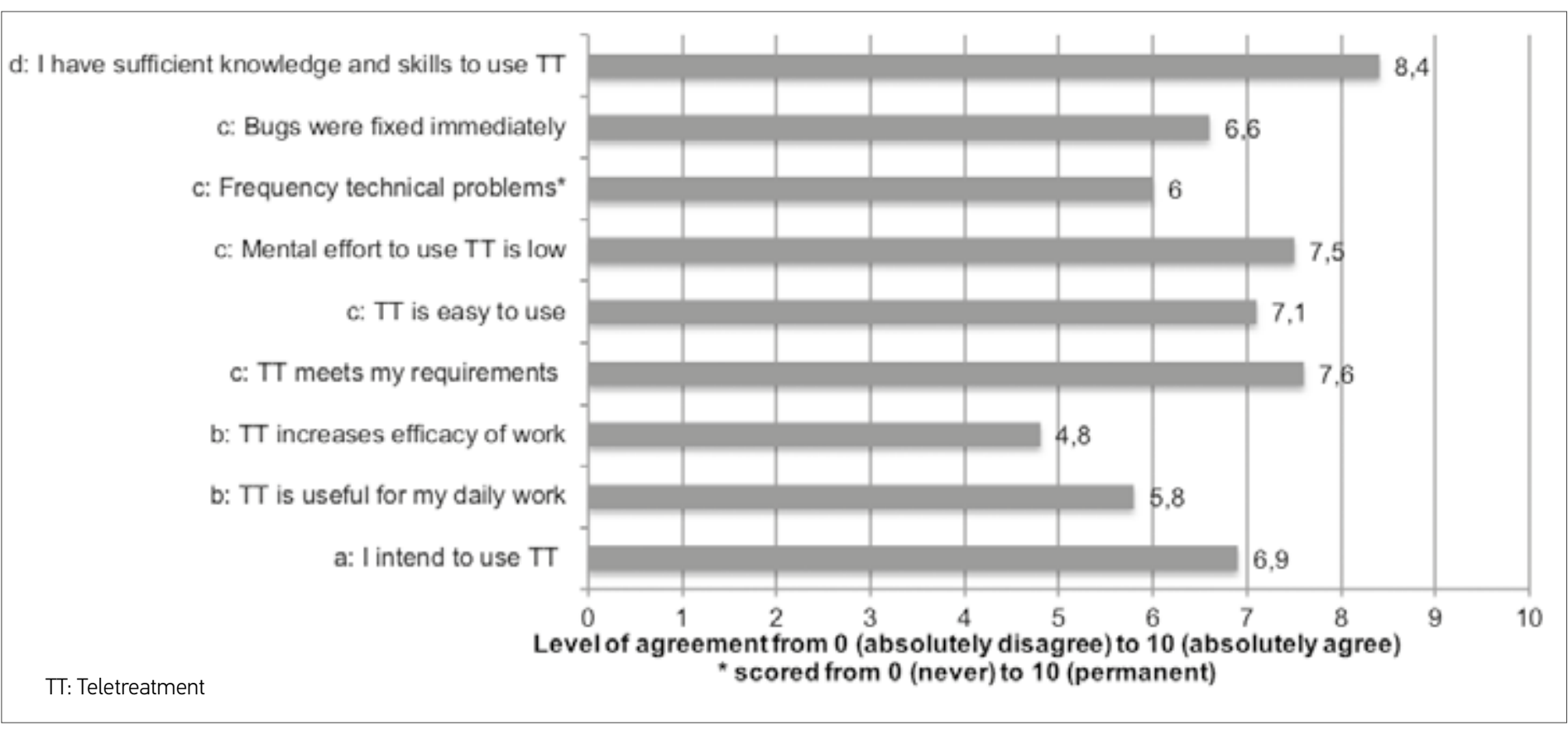

Supplemental Figure 2. Sum scores from different items of the therapist acceptance questionnaire 
Supplemental Table S1. Overall copinion of patients and suggestions for improvement regarding the teletreatment

\begin{tabular}{|c|c|}
\hline $\begin{array}{l}\text { Theme mentioned } \\
\text { by patients }(\mathbb{N} / 2 \%)\end{array}$ & Example statements \\
\hline 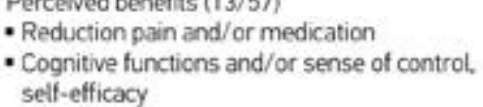 & 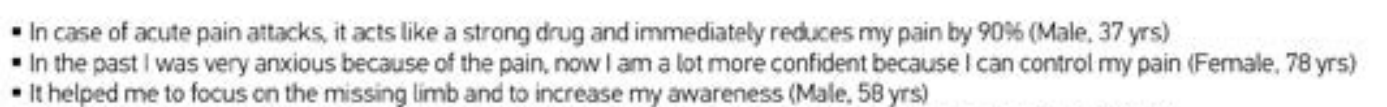 \\
\hline & ormal log Male. 33 yrs) \\
\hline 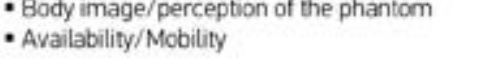 & 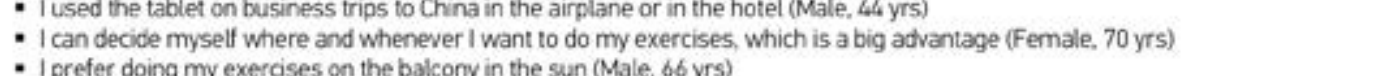 \\
\hline $\begin{array}{l}\text { - Independencer e eqarding time and place } \\
\text { - integration daly rounine }\end{array}$ & 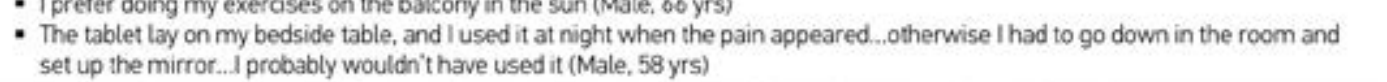 \\
\hline $\begin{array}{l}\text { Ease of use and contor } \\
\text { requirements }(9 / 39)\end{array}$ & 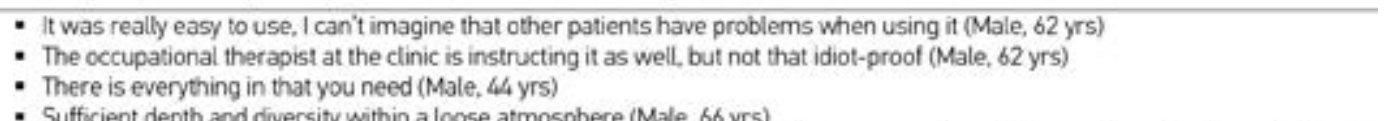 \\
\hline Providing guidance $(10 / 44)$ & 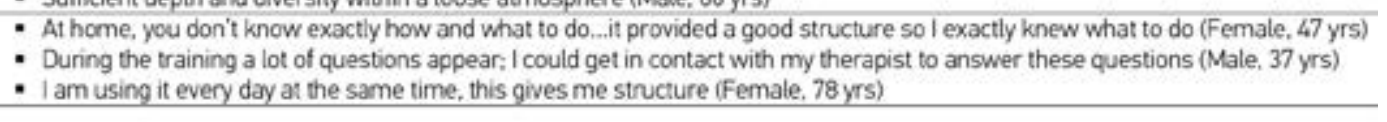 \\
\hline \multicolumn{2}{|l|}{$\begin{array}{l}\text { Aspects related to digital exercise programs } \\
\text { (111/48) }\end{array}$} \\
\hline & 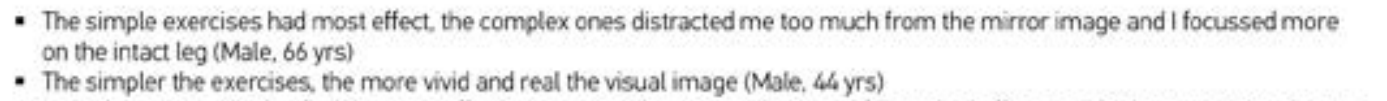 \\
\hline •Talloring & ative exercises because only some of them had effect, sol think every patient has to \\
\hline - Traditional mirror herapy vs. teletreatment & 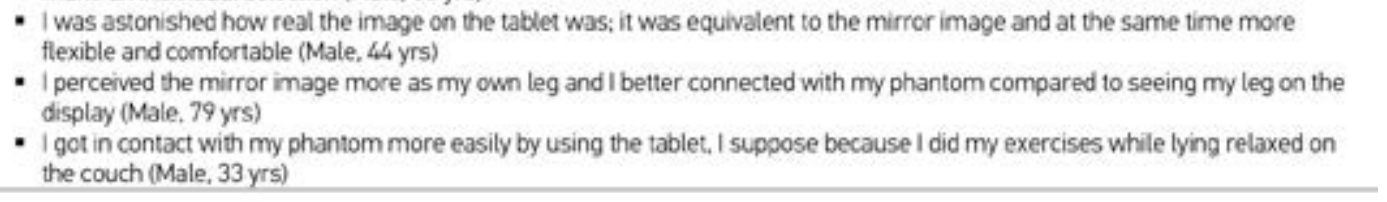 \\
\hline ms and difficultie hand & 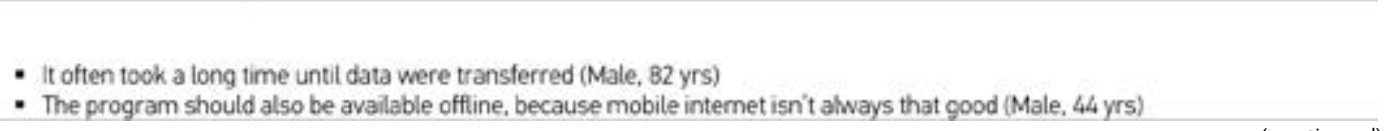 \\
\hline
\end{tabular}

Supplemental Table S1. Overall copinion of patients and suggestions for improvement regarding the teletreatment

\begin{tabular}{|c|c|}
\hline $\begin{array}{l}\text { Theme mentioned } \\
\text { by patients }(\mathbb{N} / \%) \text {. }\end{array}$ & Example statements \\
\hline - Bugs regarding e.g. exercise program & - When I chose a lot of exer \\
\hline Problems handing the tablet & 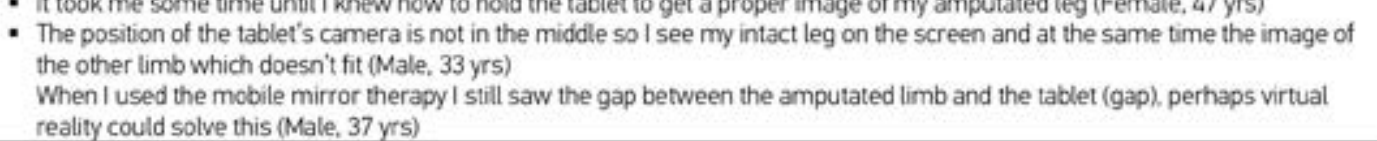 \\
\hline \multicolumn{2}{|l|}{$\begin{array}{l}\text { Instruction, personal contact and feedback } \\
(5 / 22)\end{array}$} \\
\hline introduction to teletreatment & 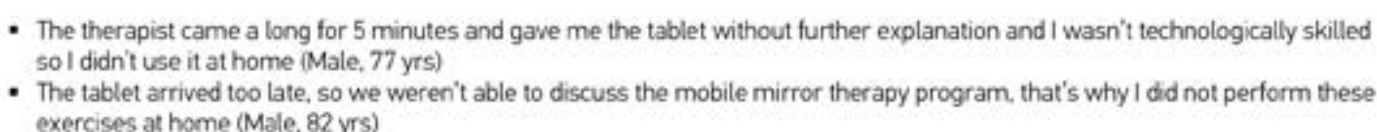 \\
\hline - Tectrical support & 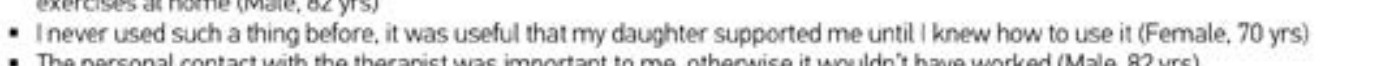 \\
\hline - Personal therapist & 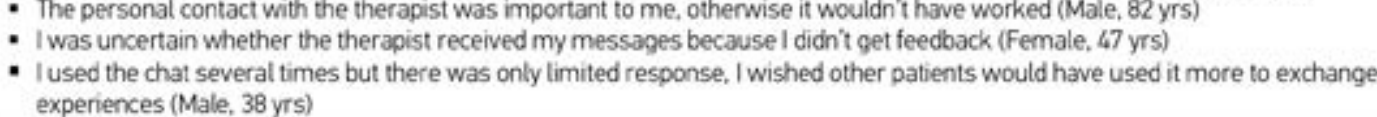 \\
\hline $\begin{array}{l}\text { Suggestions tor improvement }(7 / 30) \\
\text { vore variation in exercises }\end{array}$ & \\
\hline Dersonalize instructions & 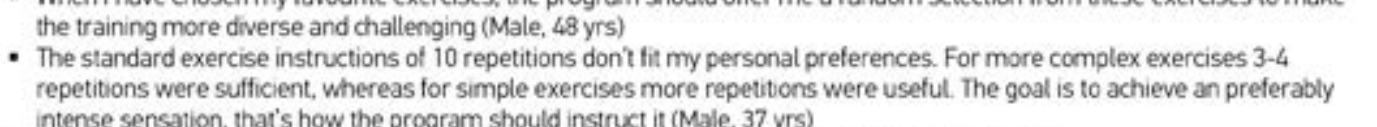 \\
\hline Nessaging & 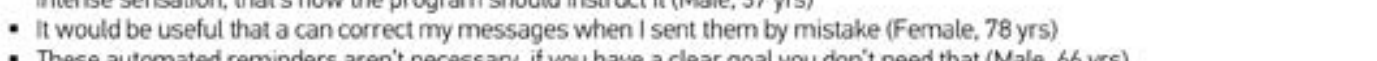 \\
\hline & These automated reminders aren't necessara, ityou have ac \\
\hline
\end{tabular}


Supplemental Table S2. Overall opinion of therapists and suggestions for improvement regarding the teletreatment Theme mentioned
by therapists (N/ $/ \%)$
Perceived benefits $(8 / 8000)$ Precticibolility
Mobility and independence of

- Work-lif balance

Self-efficacy

- Pain

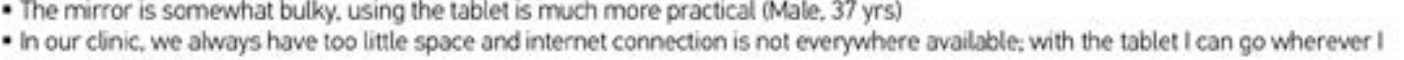
The advantage tor me re - 4h yis s)

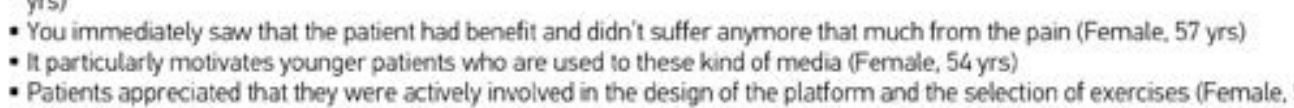

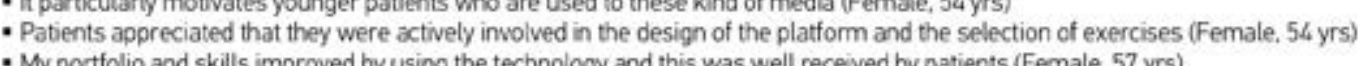

- Motivation and empowerment of

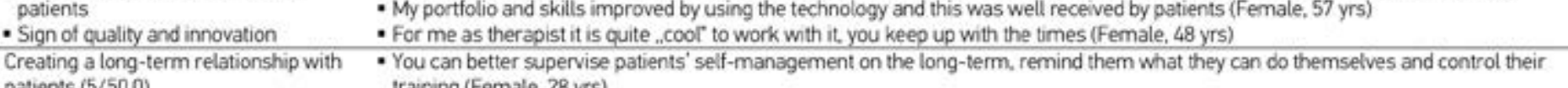

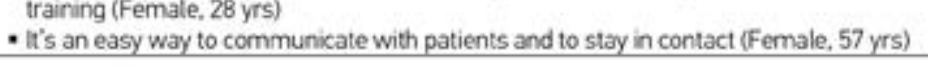

Aspects related do o digtal exercise

Programs (th

- Access 10 trearment programs

- Every patient was d
(Female, 57 yss

Weshad entect he diversitcation of exercises enabled a talored treatment program

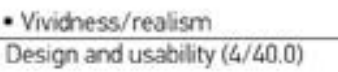

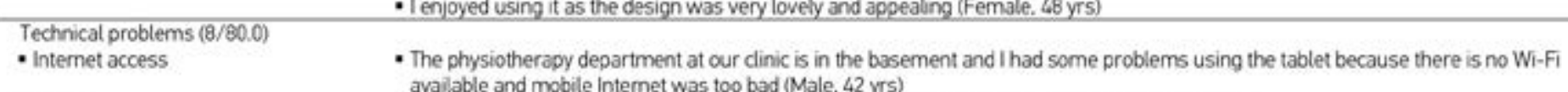
- Byusing the telet
difficult tor them

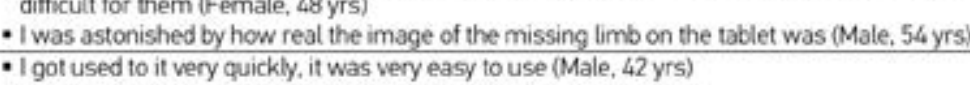

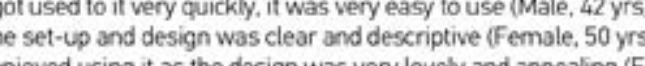

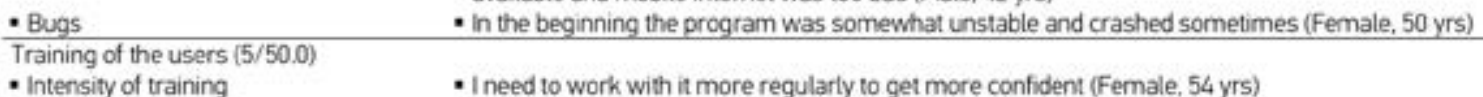

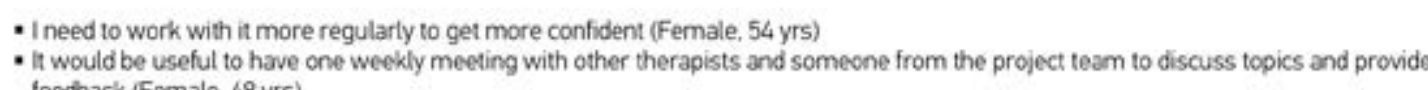

Supplemental Table S2. Overall opinion of theraists and suggestions for improvement regarding the teletreatment Theme mentioned
by therapists $(N / \%)$ Example statements

Timing of training

- Now, we were traned before the trial started, but the first patient started 8 weeks Iater because we treated just a few patients we

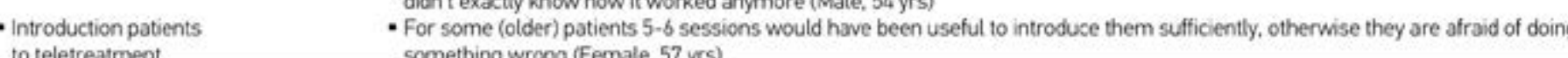

Selection of eligible patients $(3 / 30.0) \quad$ - Geriatic patients with impaired connitive functions were a bit overstrained (female, 54 y yrs)

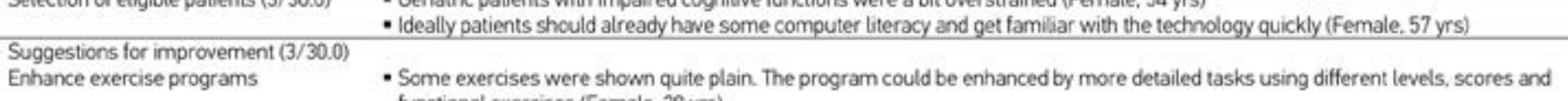

$\begin{aligned} & \text { Peer support } \\ & \text { Incorporte online community } \\ & \text { moderator }\end{aligned}$
nof

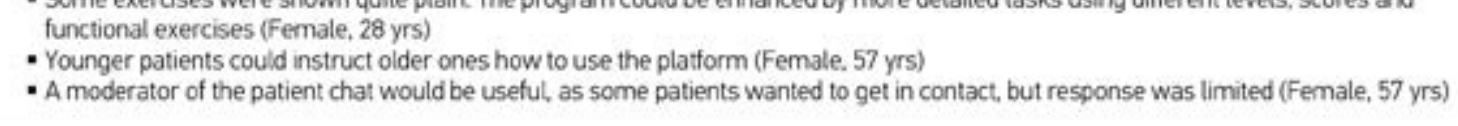




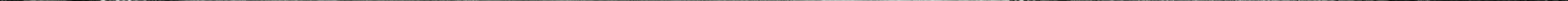


The main aim of this thesis was to develop a clinical framework for mirror therapy and a user-centered telerehabilitation platform and to evaluate their feasibility and effects in patients with phantom limb pain following lower limb amputation. The entire project entitled 'PAtient Centered Telerehabiltiation (PACT), was conducted in three consecutive phases to reach this aim. 1) creating a theoretical foundation; 2) modelling the intervention, and 3 ) evalualing the intervention in clinical practice. The objective of the first phase was to conduct a systematic review of the literature regarding important clinical aspects and the qualty of evidence of applying mirror therapy in patients with stroke, complex regional pain syndrome and phantom limb pain (Chapter 2). The aim of the second phase was to design and develop a clinical framework and a user-centered telerehabilitation platform for mirror therapy in patients with phantom limb pain following lower limb amplated in apere were evaluated in a three-arm randomized controlled trial (RCT) and an in-trial nested process evaluation (Chapters 6 and 7 ).

The final chapter first discusses the main findings related to the different phases of the project. Then, we debate several methodological aspects such as the choice of study designs and the measures used, followed by the lessons learned from the different phases of the project, which can be clustered into three topics: 1) the current evidence for mirror therapy in patients with phantom limb pain; 2) the relevance of co-designing eHealth together with different stakeholders. 3 ) the gap between theory and practice. The last section describes implications for research, clinical practice and education of future health care professionals (fig. 1).

\section{MAIN FINDINGS}

Phase I: Creating a theoretical foundation

When we started the PACC project 2010 , little was known about how to best deliver mirror therapy for patients with phantom limb pain in clinical practice and its potential effects. Therefore, a systematic review of the literature regarding the effects and clinical aspects of mirror therapy interventions was necessary to create a theoretical foundation for the subsequent phases of the project (Chapter 2). The main findings from hellerature were that he majonty of clinical trials were performed in stroke patients and only two controlled studies including a tolat of 32 amputees with phantom limb pain were published. These studies were heterogeneous regarding their design, the mirror therapy in clinical practice was sparse. Even though individual studies identifed through the literature review sugested potential

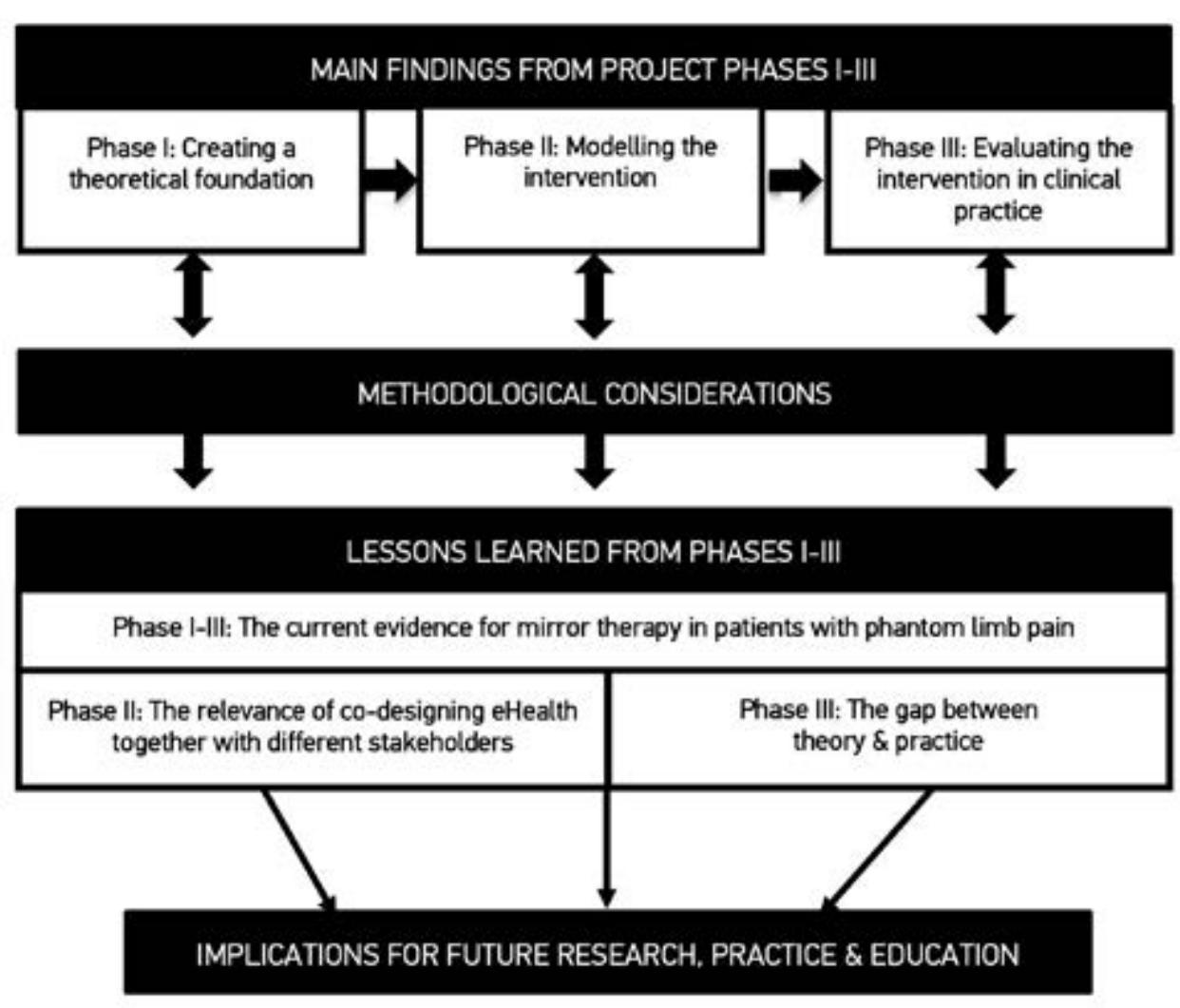

Figure 1. Overview showing the structure of the general discussion 
benefits of mirror therapy on phantom limb pain, the quality of evidence was low and important clinical aspects for the delivery of mirror therapy in clinical practice were insufficiently reported. Many different methods on how to deliver mirror therapy were described, bu detalled information and a standardized, evidence-based treatment protocol for mirror therapy in patients with stroke, phantom limb pain and complex regional pain syndrome were missing.

\section{Phase II: Modelling the intervention}

Based on the findings of our systematic review, in phase II we followed a user-centered approach to progressively model and refine the design of the interventions.' We first developed a clinical framework for traditional mirror therapy in patients with phantom limb pain to

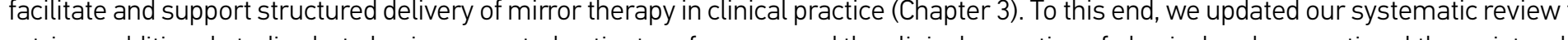
retrieve addilional studies but also incorporated palient preferences and he clincal expertse of physical and occupational herapists who

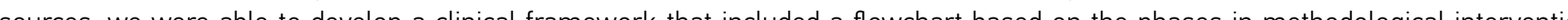

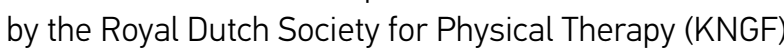

Based on the studies identified from our updated systematic review and given the chronic nature of phantom limb pain, we argued that continuous training over a period of several weeks to months seems to be needed to achieve sustainable treatment effects through mirro

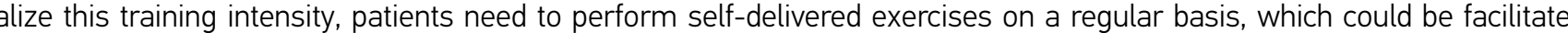
though the use of information and communication technology such as telerehabilitation.

Therefore, based on our clinical framework for traditional mirror therapy, we then developed a user-centered telerehabilitation platform exercise categories provided by the clinical framework. During the design and development of the plattorm we followed the Centre for eHeal

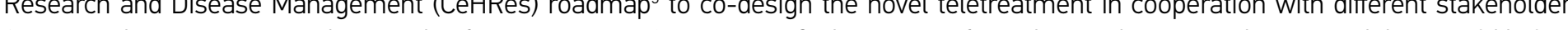
(patients, therapists, researchers and software engineers). Two main findings arose from this study: First, a decision aid that would help to priatize

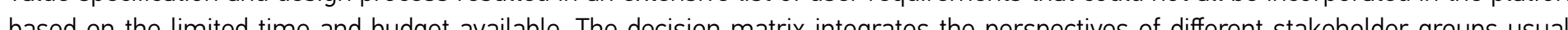
involved in eHealth projects such as the end-users, the research tean as well as desines and soffwat technicians This interprofession a proach to requirement prioritization helped improve the general stakeholder commitment and facilitated consensus about the final features se the telreatment. Second, it was crucial to involve the users and other stakeholders early and frequently in the iterative design process, so that they could give feedback on whether their requirements had been sufficiently addressed. During this design process, field testing of he technology in routine care was indispensable, in order to identify technological and contextual barriers and improve the usability of the plattorm. Overall, this systematic interprofessional approach and continuous, terative evaluation throughout was essential to developing a user-friendly high-fidelity prototype of the teletreatment.

Based on the studies performed in phase I and II, in phase III of the project we designed a three-arm multi-centre RCT (Chapter 5) to evaluate the feasibility and effects of traditional mirror therapy and the teletreatment in patients with phantom limb pain.

\section{Phase III: Evaluating the intervention in clinical practice}

and 7) showed that the framework for traditional mirror therapy in patients . in routine care (Chapter b). All groups improved over time on the majority of outcome measures, but most of the differences between the experimental and control aroups were nether statistically significant nor clinically wor mover shown in the per-protocol analysis, ie in patients receiving at least 10 sessions over 4 weeks. Furthermore, our subgroup analysis suggested significant and clinically worthwhile effects of traditional mirror therapy in women, patients with telescoping and patients perceiving a motor component (e.g. cramping) regarding the type of phantom limb pain.

The teletreatment had no additional effects on phantom limb pain compared to self-delivered mirror therapy. Only minor effects of the teletreatment over the control group were found for two of the secondary outcomes ('overall health status' and 'intrusion of phantom limb pain in daily life').

The process evaluation (Chapter 7) indicated that the interventions were not delivered as intended in the majority of patients. In most patients, the treating therapists did not deliver traditional mirror therapy according to the theoretical framework. Some therapists used parts of the mandatory sessions of tradtional miror therapy to instruct patients regarding he tedrealmen, which contibuted to insufficient treatment intensty regarding the tradilional approach. Furthermore, many therapists did not use all mandatory exercise categories from the framework In particular, elements 
Regarding the teletreatment, the findings from the process evaluation indicated that patient adherence to the teletreatment was rather low with nearly half the patients not using the teletreatment with sufficient dose. This seems to be in contrast with the findings that user accee
trial.

METHODOLOGICAL CONSIDERATIONS

\section{The following paragraph reflects on several overarching methodological aspects related to the three phases of the PACT project}

Phase l: Creating a theoretical foundation

Since little was known about the topic of mirror therapy, the literature search within our systematic review (Chapter 2) was broad and studies regarding the application and effects of miror the inclusion of recently published research. However, defining mirror therapy interventions for the literature search proved difficult because the use of a miror is jut one possible approach that creates a visualilusion Including studies that use advanced technical methods such as augmented or visual reality to create the illusion, might have added relevant knowledge to the theoretical foundation of our framework and to the design of the teletreatment in phase ll.

Regarding the study design used, it can be questioned whether a systematic review was most suitable for this relatively new treatment modality, which was characterized by a scarceness of randomized controlled trials and a sparse description of clinical aspects. Alternatively. a scoping review might have incorporated a larger range of study designs in both scientific and grey literature to address broader topics beyond those typically addressed by systematic reviews." In part, we gained insight into the grey literature and broader topics, as we already included single case studes and case series in our systematic revew and assessed elevant clicicl aspects of mintror therapy beyond intervention effeciveness alone. Thus, it remains doubtful whether performing a scoping review would have resulted in a relevant multitud of novel insights.

When we started the project in 2010, little was known about which patient groups benefit more from a mirror therapy approach. Thus, more basic research data regarding potential responders for mirror therapy might have been useful in phase I to guide patient selection for the might easily be overturned by upcoming trials and need therefore to be considered with caution. The latter also became apparent in the subgroup analysis from our RCT that suggested significant effects in patients with e.g. a telescoping phantom limb, which contrasts with 列

\section{Phase II: Modelling the intervention}

The user-centered approach that resulted in the clinical framework for mirror therapy (Chapter 3) incorporated the knowledge and opinions which was limited Furthermore, the frence in using mirror therapy. This has added relevant aspects to the data reported in the literatur intended to improve the comparability between clinical trials that evaluate the effects of mirror therapy. However, the evidence base of the framework is still weak. Also, the mirror therapy experts who took part in the development of the framework were mainly recruited via existing clinical networks of the principal investigator, bearing the risk of selection bias.

The framework served as a departure point for the development of prototypes of the telerehabilitation platform (Chapter 4), which were tested in real-life situations together with the end-users through continuous evaluation cycles. This participatory user-centered approach crucially The users were able to check whether their requirements had been sufficiently addressed, and, based on their feedback some features were rejected because the users did not consider them relevant. Furthermore, during development of the teletreatment we incorporated facilitators for long-lerm engagement and user motivation. ${ }^{18}$ The decision matrix that was developed and used during the design of the telerehabilitation platform helped prioritize the user requirements. It however also bears the risk that some potentially relevant features the

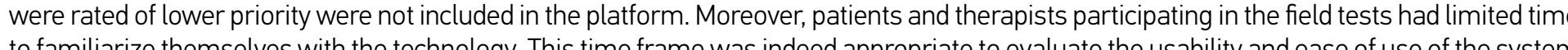
(inglementation in daily practice.

When we designed the RCT (Chapter 5), little was known about which patient groups might benefit best from mirror therapy. Therefore, we kept selection criteria for the trial as pragmatic as possible and included a patient group that was heterogeneous regarding differen characteristics such as age, reason of amputation or the duration of symptoms. We also included patients who had received mirror therapy in the past, if not more than six reatments had been delivered during the previous three months before trial inclusion. This cut-off was chosen

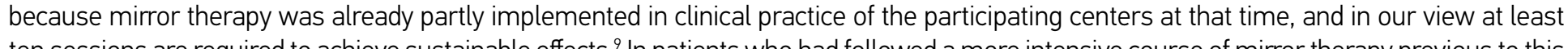
time frame of three months, possible effects of the intervention should have faded However we did not check the response of these patients to the previous course of mirror therapy, which implies the risk that potential non-responders might also have been included in the trial. 
Furthermore, no restriction was made regarding the time since amputation, resulting in a large variation of participants who had been

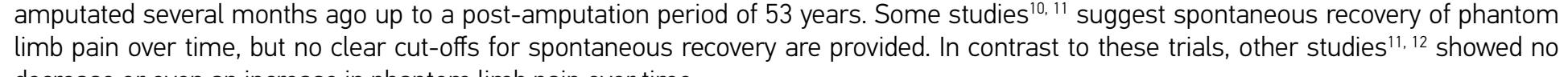
decrease or even an increase in phantom limb pain over time.

In the RCT presented in this thesis, spontaneous recovery of phantom limb pain is unlikely, since the median time post amputation of on their capacity to engage in the mirror illusion and to relate the mirrored reflection to their phantom limb One study's suggested that this capacity might predict the treatment effect. However, based on the small sample size of this study and the possibility that this capacity changes over time, we decided not to add this aspect to the selection criteria.

Phase III: Evaluating the intervention in clinical practice

Our randomized controlled trial that evaluated the effects of the novel interventions in clinical practice (Chapter 6) was carefully planned Measurements, and Pain Assessmentin Clinical trials) recommend o Rep 14 We included and trained many different centers and os Methods, of strategies to facilitate patient recruitment 15 Our goal was to increase the likelihood that a representative population for the rehabilitation practice in Germany would be included and to enhance the generalizability of the interventions and the trial results. Furthermore, our trial is at present the largest RCT on mirror therapy for patients with phantom limb pain with a long-term follow-up at six months.

The limited effects found in the clinical trial might partly be explained by the insufficient sample size (power) and contrast between the groups and the finding th tound in the

weak as well as the added value of the teletreatment compared to the traditional approach. Since our trial didr not reach the calculidence was size, this design choice resulted in an even smaller sample size when the three groups were analyzed separately regarding the effects of the teletreatment following the first 4 weeks of traditional mirror therapy. Furthermore, large placebo analgesic effects by offering a control intervention have been reported in pan palients, in particular in pallents with neuropathic pain including phantom limb pain. " This might have diminished the contrast between the experimental and control groups. As an additional factor, co-interventions might have influenced the contrast between the groups, but we did not monitor for these during the trial. Finally, the triat was powered to detect a two-point difference 作 been proposed as being clinically relevant for patients suffering from phantom limb pain.
One reason why the clinical framework for traditional mirror therapy was not adequately delivered might be that the design of our multicenter RCY involved nine different centers which increased fragmentation: Some of the therapists felt uncomfortable in delivering the interventions, in particular regarding the teletrealment, and experienced difficulties in becoming sufficiently skilled because of the small numbers of palients they treated. However, the inclusion of many centers was necessary to achieve the calculated sample size within a given the frame, hat can also be seen in the design of asurenlly ren ch the clinical framework during the first four weeks and the additional effects of the teletreatment following the first four weeks of traditional mirror therapy patients allocated to the teletreatment group were not introduced to the technology before the fourth week of treatment. One mirror therapy and thus might have felt no need to further use the telerreatment during the subsequent study period. This also introduces the risk of selection bias, and it probably might have been useful to only include patients in the teletreatment group who further needed to

As seem to be less suitable to evaluate the impact of complex, disruptive interventions such as eHealth, 319-23 Thus, it can be questioned whether the RCT conducted in the present thesis was best suitable to address the effects of the teletreatment, or whether other study designs, which will further be outlined below in the "lessons learned" paragraph migh more appropriate. The in-trial nested process evaluation presented in this thesis used quantitative and qualitative methods to collect data from multiple sources
regarding the delivery of the interventions in clinical practice. However, these data were analyzzed after the completion of the trial. Some

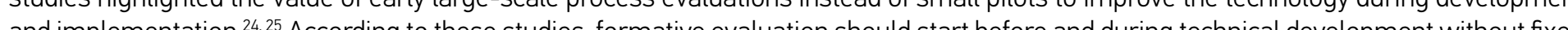
end, as the technology fluctuates over time. Therefore, the process evaluation presented in this thesis might already have provided useful insights during phase Il of the project before full-scale evaluation took place.

\section{LESSONS LEARNED}

In the following paragraph, the lessons learned based on the methodological considerations from the three phases of the PACT project will

Phase I-III: The current evidence for mirror therapy in patients with phantom limb pain

Below, we will outtine the current evidence for mirror therapy in patients with phantom limb pain by presenting how the studies from the 
Despite the limited evidence identified in our systematic review in phase I, traditional mirror therapy was already accepted and implemented by many professionals in clinical practice without following a standardized protocol. At the same time, a huge demand existed fom profossionals on how to structure and deliver the intervention in clinical practice based on the current evidence. Therefore, we first developed a protocol for mirror therapy in stroke patients in 2013 , based on the best available evidence at the time, patient preferences and pros. 30.000 reads by healt
and published it open access. ${ }^{2}$ Five years after its online publication the protocol has already reached more than and published it open access. ${ }^{6}$ Five years after its online publication the protocol has already reached
care professionals, researchers and educators around the world. Given this huge demand from clinical practice and in preparation of our randomized controlled trial, we decided to develop a similar framework for mirror therapy in patients with phantom limb pain. The first pillar that guided the development of the clinical framework in phase II was the best evidence available at that moment, which lead to an updare of our systematic review to retrieve additional studies that were published from August 2010 through June 2014. However, no additional controlled clinical trials since the publication of our systematic review were identified. In the third phase of the project, our RCT did not reveal significant effects of mirror therapy on the averase
suggested in the per-protocol and subgroup analysis.

While we conducted our RCT, the results of three other randomized controlled studies ${ }^{27-29}$ investigating effects of mirror therapy in patients . The sample sizes ranged from $15^{28}$ to 64 anp no significant effect. More recently, results from a three-group cross-over study in 45 landmine victims from Cambodia were published. The research population consisted of patients with chronic phantom limb pain with an average post-amputation time of 23 years. This study reported postive effects of four-weeks mirror therapy in combination with tactile therapy of the stump on the average phantom limb pal difference between the threek (measured on a comparing the results from recently published trials and the results from the RCT presented in this thesis: Only studies that used intervention periods of several week ${ }^{2-300}$ showed effects on phantom limb pain. In our RCT, only patients who adhered to the predefined treatment protocol and followed at least 10 sessions over four weeks showed a significant treatment effect on the primary outcome after four weeks. Thus, a centon teat ment intensily seents to be mandatory to achieve resuls. A recent study" suggested that patients with more severe phantom limb pain require an even higher treatment intensity of up to 21 sessions to obtain pain relief. The recently published studies ${ }^{27-31}$ Sur subgroup analysis

reported in some studies the evidudies in this thesis and recently published trials, it seems that despite promising benefits of mirror therapy evidence regarding which patients might best benefit from this approach is inconclusive.
Phase II: The relevance of co-designing eHealth together with different stakeholders

of the interventions in phase II of the project.

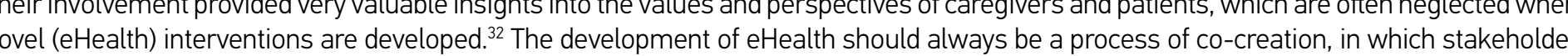
novel (eHealth) interventions are developed. ${ }^{32}$ The development of eHealth should always be a process of co-creation, in which stakeholder know and understand each other. The different stakeholders need time to learn and speak each other's language and to understand the different visions and needs. This interprofessional and user-centered design process resulted in a high-fidelity prototype of the teletreatment that demonstrated sufficient user acceptance rates in phase three of the project. However, thorough stakeholder involvement made the design process time-consuming and does not automatically lead to adoption and implementation of the technology in routine care. Since technology development is interwined with its implementation, sufficient time should be allotted for thorough pilot testing and training of the users, so that potential implementation issues such as limited resources and integration in established care processes can be identified as early as during the pilot phase and not only after fult-scale evaluation. $3 ., 35$ The field-testing performed in routine care might therefore

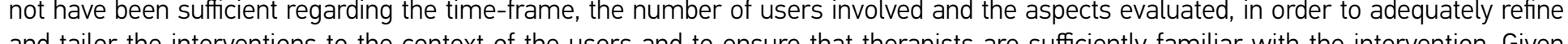
政 expand the time period for implementation and adoption of the teletreatment as well as recruitment of patients for the RCT.

Clearly, the implementation and adoption of eHealth in routine care remains a major challenge, as these technological solutions are in many The most rection health care professionals is laging especially when considering the ambitious goals set for the coming years $40 \mathrm{In}$ particular for applications where both the healthcare professional and the healthcare user have a role to fulfill, when the added value is unclear or when the application requires a change of the existing routine care process, less progress is observed."

Phase III: The gap between theory and practice

作 dill of the project Only $31 \%$ of patients received the mandatory norts of thatically result in large patient adherence rates and effects in phase patients used the teletreatment with sufficient dose according to the protocol after the first four weeks. 
interventions such as eHealth. However, RCTs are still considered the gold standard for assessing the effectiveness of complex interventions
as it best eliminates potential sources of bias, such as selection bias and confounding, by carefully selecting patients and using stric methodology." "Several aspects, such as the heterogeneity of patients and the complexity of chronic pain itsell, can hardly be standardized

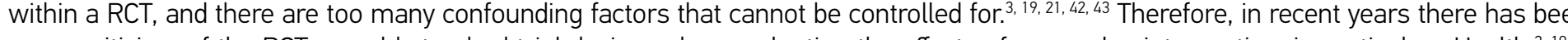
some chCT and implemented with high fidelity and will most likely achieve clinically meaningful benefits 4 These interventions need to be sufficiently tested already and implemented in routine care to ensure that health care professionals have sufficient experience in using these interventions. For novel disruptive interventions such as eHealth that are not yet adopted by health care professionals, RCTs seem to be less suitable and 列 in this thesis.

Regarding the choice of outcome measures, the selection of the average phantom limb pain intensity during the previous week as primary U. goals within single case experimental designs might be a better alte
which would be more in line with patient centered routine care. 4,48

From the evaluation and implementation of the interventions in clinical practice we learned that many therapists experienced high time pressure in their daily care process, which probably results in a lack of time to perform extra tasks needed to properly adopt' novel' intervention adapt the clinical framework and to instruct and train patients how to use the teletreatment in order to facilitate succesfful implementation daily life.49,50 In particular, components of the clinical framework such as mental practice that were not already part of professionals' routine before the trial were less or nol at all delvered. Regarding the use of teletreatnens, a recent swdy showed hat he technology should faciltiale practice amongst sufficiently skilled and experienced in using the technol mogy before full-scale evaluation took place. More intense support in terms of training and roubleshooting regarding the use of the teletreatment before, during and atter their implementation might have improved adherence and

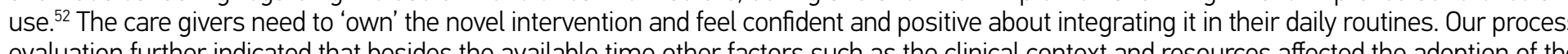
(it use of eHealth in routine care ${ }^{53,4}$
IMPLICATIONS FOR FUTURE RESEARCH, PRACTICE \& EDUCATION professionals based on the lessons learned from this project.

Implications for Research

will insufficient There is 1 s demonstrate the potential benefits of traditional mirror therapy to reduce phantom limb pain, the evidence is variety of recruitment strategies ${ }^{15}$ to include sufficient patients. However, before additional multicenter trials are conducted, further research on which patients are more likely to benefit from mirror therapy is required; as our trial and recently published studies pointed out, a better response to mirror therapy might depend on several patient characteristics, such as the level of pain intensity ${ }^{3}$ the type of phantom limb
pain ${ }^{5.55}$ telescoping pain, 5,56 telescoping,
importance of and gender. towards a more personalized treatment. ${ }^{2,4}, 47$ More recently, technological alternatives such as augmented and virtual reality setups have been proposed for the treatment of patients with phantom limb pain. ${ }^{77-59}$ However, until now, little is known about important clinical aspects and effects of these alternatives to traditional mirror therapy, but larger studies are ongoing. ${ }^{18}$ Thus, in the near future, such advanced technical solutitons might also contribute to a personalized treatment of patients with phantom limb pain should they have proven to achieve sufficient
effects. Future studies need to identify responders for each approach to facilitate a personalized treatment based on patient characteristics,

Further research is needed regarding how technology can efficiently support daily care processes of health care professionals and which aspects are crucial for successful implementation and adoption of eHealth in routine care. The Dutch eHealth Monitor 2018 pointed out tha little trust seems to exist amongsthealth care professionals regarding the positive effects of eHealth and patients' capacity to correctly interpret results and estimate privacy risks. . $^{39}$ Therefore, we need more research and focus regarding critical aspects for technology adherence and
acceptance such as 'trust,' which is seen as an important element for end-user acceptance. . $^{1,62}$ At the moment, our knowledge about these aspects is very limited.

Furthermore, future trials investigating the impact and effects of telehealth need to consider alternative research designs to the RCT paradigm that better mach he principle of personalzed care and he complex and discupive halure of ellealh. be improved and adapted varly during the development process (eg through log file analysis ${ }^{63}$ ). so that the technology better suits the clinical context of routine care which might improve the outcomes of full-scale evaluation. 
Implications for clinical practice

The treatment of chronic (phantom limb) pain in clinical practice remains challenging. ${ }^{21}$ Despite insufficient evidence that mirror therapy is effective in reducing phantom limb pain, mirror therapy is partly implemented in routine care, because non-pharmacological treatment
options are limited and many therapists reported benefits of using mirror therapy in treating phantom pain in amputees. . $^{64}$ Amongst the options are limited and many therapists reported benefits of using mirror therapy in treating phantom pain in amputees. ${ }^{4}$ Amongst the
different non-pharmacological options to treat phantom limb pain, the evidence for mirror therapy is at present better than for other potentia different non-pharmacological options to treat phantom limb pain, the evidence for mirror therapy is at present better than for other potenti practice and ris commended that health care professionals should base their choices for a particular treatment more on the clinical contex as well as patient preferences and needs (in particular when strong evidence is missing). ${ }^{41}$ In the light of limited effects and potential adverse out some important aspects that should be considered when delivering mirror therapy in clinical practice:

- As described in our clinical framework, health care professionals need to carefully select

patients based on their characteristics such as the condition of the intact limb or the mental state.
- Mirror therapy needs to be performed with sufficient dose, that is at least 10 sessions up to 21

sessions over 4 weeks.

- The intervention characteristics of mirror therapy such as the treatment dose and exercises

used should be tailored to the features and preferences of the individual patient. - The delivery and effects of mirror therapy should be mos
outcome measures related to pain and individual goals.

Eligible patients should decide together with their treating therapist whether they are willing to engage in a course of 10 mirror therapy sessions and self-delivered exercises to evaluate their response to the treatment. Therapists might use the clinical framework for mirror therapy described in the present thesis to structure the intervention and to facilitate self-delivered exercises of patients. At the end of the course of 10 sessions, the therapist should discuss the outcome of the treatment with the patient and whether further self-delivered exercises are needed after discharge. If this is the case, the therapist might evaluate patients' eligibility to use the teletreatment and discuss with the
patient if she/he would like to use it as additional tool to support self-delivered exercises. Furthermore patients who are not able to visit the therapist several times in person, might be offered the possibility to use the teletreatment to enable self-management.
thent

The implementation of telehealth applications in clinical practice remains challenging as many different aspects besides the intervention such
as user characteristics, skills and context influence their adoption.97,70 Due to an ageing population and increasing number of people with chronic conditions, health care costs will rise in the coming years, whereas a shortage of healthcare employees is expected. ${ }^{39}$ Therefore, national to create a personalized blended health service and to facilitanted in this thesis could be used in combination with face-to-face sessions face-to-face and teletreatment sessions needs to be personalized to individual patient needs, preferences and characteristics. ${ }^{69}$ The time and training needed by the users to become confident and sufficiently skilled in using the teletreatment will also depend on user characteristics and their context.

Despite these expectations and the hope that eHealth will solve a part of the future problems of our health care systems, the actual use of

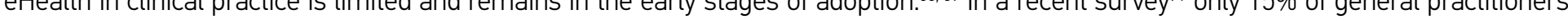
reported a use of telehealth. Two top barriers to wider adoption of telehealth were identified by the survey: (1) insufficient information and training of professionals regarding the use of telehealth services and (2) lack of reimbursement of telehealth services. Thus, to foster the effective eHealth interventions that tackle this major barrier experienced by the majority of health care professionals. Initial tendencies in this direction became apparent in the recent years. In Germany e g some insurance companies pave the way for the implementation of novel telerehabilitation concepts by publishing a framework about their practical requirements and potential reimbursement. ${ }^{72}$ In the Netherlands, the Dutch Healthcare Authority recently published a guideline on the reimbursement of different eHealth services to facilitate their adoption

in clinical practice.
Although health insurance companies are an important stakeholder regarding the wider adoption of eHealth, alternative business models also have to be considered. Future eHealth interventions should incorporate business modelling that includes a concrete business case and a models that include more than one source for payment such as health insurance companies, local government and end-users. Health insurance companies need to be aware of the potential limits of RCTs in evaluating the impact and effects of eHealth, which now still serve as the standard regarding reimbursement policies. Alternative research designs described above in the 'lessons learned' paragraph should be considered.

The use of information and communication technology also raises ethical and policy challenges, as it radically changes the way of when
where and how patients and health care professionals engage with one another ${ }^{76}$ On the one hand telehealth has the potential to benefit patients, on the other hand it disupts the relsionship between patint and care continuously evolving and changing the way health care is delivered the fundamental ethical responsibilities of health care professionals and other stakeholders have not changed. In any model of care, health care professionals have the responsibility to deliver competent care, 
provide patients
other interests.

Digital health represents a data ecosystem in which vast amounts of medical data are gathered through multiple sources such as electronic health records, companies in the private sector or the patients themselves. In recent years, increasingly large data sets that include sensitive includes lifestyle data and suchs to electic more and more data from the users of health-related services and products such as the genetic testing company 23 andMe who collected and genotyped DNA from more than one million customers in $2015^{80}$ Moreover big technology companies such as Google are joining forces with companies from the medical field such as Sanofi, in creating new care and business models e.g. for patients with diabetes. ${ }^{81}$ Finally, the end-users of digital health devices are increasingly taking control of their medical data and contribute vast amounts of data to service providers through wearables and fitness gadgets. Looking ahead, the number of large-scale data sources will further increase and advanced big dala analytical meliods will be applied, making protection of dala privacy, confidentially and elical principles increasingly complex. The question could be addressed by eg developing new definitions and standards based on privacy science and the expectations of the intended A culture of trust regarding digital healthneeds to be established, which requires aspects beyond privacy protection alone, such as transparency accountability, benefit sharing and clarity about data ownership. ${ }^{78} \mathrm{Health}$ care professionals play an important role to guide patients and tel them why, where and how eHealth is used so that patients are sufficiently informed and perceive trust. Patients need to be informed tha different aspot replace face-to-face and hands-on therapy - a fear that in particular older patients have ${ }^{\mathrm{e}}$ - but rather complement it. These me aspects of future) ellealth applications must critically be evaluated with appropriate tools, and measures should be taken before wider deployment

Implications for education of (future) health care professionals
The treatment of patients with chronic pain and other chronic disorders is a complex and interprofessional issue and requires a holistic and interprofessional approach to education of (future) health care professionals. Students need to learn how to work together efficiently with limb pain the spectrum of non-pharmacological treatment options and their evidence should be integrated in the education of future health care professionals. The framework for mirror therapy as well as important clinical aspects of the intervention, as described above in the implications for clinical practice, should be discussed with students to support them in delivering a personalized treatment. Moreover, the framework could serve as guideline for students how to structure the intervention.

Regarding the implementation of eHealth in the education of future health care professionals, the 2018 Dutch eHealth monitoris showed that the majority of (future) healhcare providers feels digitally skilled, but that hine range on technical possibities is not always known. Many universities have not ye systematically integrated eHealth and aspects regarding technology acceptance into their curricula, despite a generation of students that already grew up with these technologies and is digitally skilled. Many lecturers do not feel sufficiently informed process andeducation Thus, there is need for sufficientinformation and training of lecturers and students, in order to enable (future) healthcare providers to offer and use eHealth in routine care: awareness for the potential advantages, limitations and failures of using eHealth may be raised by sharing the lessons learned from different eHealth projects and experiences amongst healthcare providers working with eHealth. In particular, eHealth experience with patient access is still limited and uptake is low.39 Therefore, sharing positive and negative experiences about patient access to eHealth with students from different faculties, teachers and health care professionals would provide important insights and knowledge, which would in turn facilitate further development and upscaling of eHealth. "In addition, it is important to provide
(future) health care professionals with insights regarding the significance of a interprofessional and user-centered eHealth design process by using tools such as the CeHRes roadmap 3 in eduction and training. Digital health technologies that are already available and might be of clinical relevance for future health care professionals should be identified, and their quality should be assessed with appropriate tools and measures.5.5 High-quality and effective technologies should then be integrated into the education of (future) health care professionals while taking important features such as the patient perspective, legal and ethical aspects into account. ${ }^{78,85,86}$ 


\section{REFERENCES}

Craig P, Dieppe P, Macintyre S, Michie S, Nazareth I and Petticrew M. Developing and evaluating complex interventions: the new Medical Research Council guidance. BMJ. 2008; 337: al 655.

de Vries C, Hagenaars L, Kiers $\mathrm{H}$ and Schmitt M. The professional profile of the physical therapist

Amersfoort: Royal Dutch Society for Physical Therapy, 2006.

van Gemert-Pijnen JE, Nijland N, van Limburg M, et al. A holistic framework to improve the uptake and

impact of eHealth technologies. J Med Internet Res. 2011; 13: e111.

Arksey $\mathrm{H}$ and O'Malley L. Scoping studies: towards a methodological framework. International Journal of Social Research Methodology. 2005; 8: 19-32.

Levac D, Colquhoun H and O'Brien KK. Scoping studies: advancing the methodology. Implement Sci.

2010; 5: 69.

Foell J, Bekrater-Bodmann R, Diers M and Flor H. Mirror therapy for phantom limb pain: brain changes and the role of body representation. Eur J Pain. 2014; 18: 729-39.

Nifland N, van Gemert-Pfinen JE, Kelders SM, Brandenburg BJ and Seydel ER. Factors influencing the use of a Web-based application for supporting the self-care of patients with type 2 diabetes: a longitudina study. J Med Internet Res. 2011; 13: e71.

Oinas-Kukkonen H and Harjumaa M. Persuasive Systems Design: Key Issues, Process Model, and System Features. Commun Assoc Inf Syst 2009; 24: 28

Seidel S, Kasprian G, Furtner J, et al. Mirror therapy in lower limb amputees--a look beyond primary motor cortex reorganization. RoFo : Fortschritte auf dem Gebiete der Rontgenstrahlen und der Nuklearmedizin. 2011; 183: 1051-7.

10. Houghton AD, Nicholls G, Houghton AL, Saadah E and McColl L. Phantom pain: natural history and association in rehabilitation. Ann R Coll Surg Engl. 1994; 76: 22-5.

11. Nikolajsen L, Ilkjaer S, Kroner K, Christensen JH and Jensen TS. The influence of preamputation pain on postamputation stump and Khantom pain. Pain 1997: 72: 393-405.
12. Richardson C, Crawford K, Milnes K, Bouch E and Kulkarni J. A Clinical Evaluation of Postamputation Phenomena Including Phantom Limb Pain after Lower Limb Amputation in Dysvascular Patients. Pain Manag Nurs. 2015; 16: 561-9.

13. Boutron I, Moher D, Altman DG, Schulz KF, Ravaud P and Group C. Extending the CONSORT statement to randomized trials of nonpharmacologic treatment: explanation and elaboration. Ann Intern Med. 2008; 148: 295-309

14. Dworkin RH, Turk DC, Farrar $J$, et al. Core outcome measures for chronic pain clinical trials: IMMPACT recommendations. Pain. 2005; 113: 9-19.

15. Pinto CB, Velez $F S S$, French MN, et al. Strategies to Enhance Recruitment Methods in Phantom Limb Pain Clinical Trials. Int J Cin Trials. 2017; 4: 72-9.

16. Vase L, Skyt I and Hall KT. Placebo, nocebo, and neuropathic pain. Pain. 2016; 157 Suppl 1: S98-105.

17. Smith DG, Ehde DM, Hanley MA, et al. Efficacy of gabapentin in treating chronic phantom limb and residual limb pain. J Rehabil Res Dev. 2005; 42: 645-54.

18. Lendaro E, Hermansson L, Burger $\mathrm{H}$, et al. Phantom motor execution as a treatment for phantom limb pain: protocol of an international, double-blind, randomised controlled clinical trial. BMJ Open. 2018; 8: e021039.

9. Bothwell LE, Greene JA, Podolsky SH and Jones DS. Assessing the Gold Standard--Lessons from the History of RCTs. N Engl J Med. 2016; 374: 2175-81.

Kibbelaar RE, Oortgiesen BE, van der Wal-Oost AM, et al. Bridging the gap between the randomised clinical trial world and the real world by combination of population-based registry and electronic heal

. Pain. 2013; 154: 1929-31.

22. Pagliari C. Design and evaluation in eHealth: challenges and implications for an interdisciplinary field. J

22.

Comput Biol Med. 2002; 32: 209-20. 
24. Van Der Meijden MJ, Tange HJ, Troost J and Hasman A. Determinants of success of inpatient clinical information systems: a literature review. J Am Med Inform Assoc. 2003; 10: 235-43.

25. Eng TR, Gustafson DH, Henderson J, Jimison H and Patrick K. Introduction to evaluation of interactive health communication applications. Science Panel on Interactive Communication and Health. Am J Prev Med. 1999; 16: 10-5.

26. Rothgangel AS and Braun SM. Mirror Therapy: Practical Protocol for Stroke Rehabilitation. Munich. Pflaum Verlag, 2013.

27. Tilak M, Isaac SA, Fleicher J, et al. Mirror Therapy and Transcutaneous Electrical Nerve Stimulation for Management of Phantom Limb Pain in Amputees - A Single Blinded Randomized Controlled Trial. Physiother Res Int. 2016; 21: 109-15.

28. Finn SB, Perry BN, Clasing JE, et al. A Randomized, Controlled Trial of Mirror Therapy for Upper Extremity Phantom Limb Pain in Male Amputees. Front Neurol. 2017; 8: 267.

29. Ramadugu S, Nagabushnam SC, KatuWal $N$ and Chatterjee K. Intervention for phantom limb pain: A randomized single crossover study of mirror therapy. Indian J Psychiatry. 2017; 59: 457-64,

Ol HS, Van Heng $Y$, Danielsson L and HUSH Mirror therapy for phantom limb and stump pain: a randomized controlled clinical trial in landmine amputees in Cambodia. Scand J Pain. 2018; 18: 603-610. Griffin SC, Curran S, Chan AWY, et al. Trajectory of phantom limb pain relief using mirror therapy: Retrospective analysis of two studies. Scand J Pain. 2017; 15:98-103.

2. Bertoncello C, Colucci M, Baldovin T, Buja A and Baldo V. How does it work? Factors involved in

33. Van De Belt TH, Engelen LJ, Berben SA and Schoonhoven L. Definition of Health 20 , 3 . Medicie. Van De Belt TH, Engelen LJ, Berben SA and Sch A reon

Broens TH, Huis in't Veld RM, Vollenbroek-Hutten MM, Hermens HJ, van Halteren AT and Nieuwenhuis Determinants of successful telemedicine implementations: a literature study.J Telemed Telecare 2007: 13: 303-9.

35. Ovretveit J, Scott T, Rundall TG, Shortell SM and Brommels M. Improving quality through effective implementation of information technology in healthcare. Int J Qual Health Care. 2007; 19: 259-66.
36. Ribeiro C, Farinha C, Pereira J and Mira da Silva M. Gamifying requirement elicitation: Practical implications and outcomes in improving stakeholders collaboration. Entertain Comput. 2014; 5: 335-45.

Ghanbari H, Simila J and Markkula J. Utilizing online serious games to facilitate distributed requirements elicitation. J Syst Softw. 2015: 109: 32-49.

38. Wouters M, Swinkels I, Sinnige J, et al. Consciously choose eHealth- summary eHealth-monitor. Den Haag/Utrecht: Nictiz, NIVEL, 2017

39. Wouters M, Swinkels, I., Lettow, B. van, Jong, J. de, Sinnige, J., Brabers, A., Friele, R., Gennip, L.van. EHealth at different speeds: a brief outline eHealth-monitor 2018. Den Haag/Utrecht: Nictiz, NIVEL, 2018

40. Krijgsman J, Peeters, J, Waverijn, G., Van Lettow, B, Van der Hoek, L, De Jong, J. Friele R, Van Gennip, $L$. 'Because I think it is important to take proper care of myself' 2016 Report on eHealth Objectives. The Hague \& Utrecht: Nictiz \& NIVEL, 2016.

41. Raad voor Volksgezondheid en Samenleving. Zonder context geen bewijs: Over de illusie van evidencebased practice in de zorg. Den Haag 2017.

42. Glasgow RE. eHealth evaluation and dissemination research. Am J Prev Med. 2007; 32: S119-26.

43. ResnicowK Strecher V, Couper M, et al Methodologic and design issues in patient-centerede-health research. Am J Prev Med. 2010; 38: 98-102.

Murray E Hekler EB, Andersson 6 , et al Evalua

Approaches Am J Prev Med 2016, 51:8/3-51.

Lawer MS and D'Agostino RB, Sr The randomize

Iized registry trial--the next disruptive technology in clinical research? N Engl J Med. 2013: 369: 1579-81.

46. Vlaeyen J Morley S, Linton SJ, Boersma K and de Jong J. Pain-related fear : exposure based treatment for chronic pain. 1 ed. Seattle (WA): IASP Press, 2012, p.196

4. Onghena P and Edgington ES. Customization of pain treatments: single-case design and analysis. Clin $」$ Pain. 2005; 21: 56-68; discussion 9-72.

Smith JD. Single-case experimental designs: a systematic review of published research and current standards. Psychol Methods. 2012; 17: 510-50. 
49. Ariens LF, Schussler-Raymakers FM, Frima C, et al. Barriers and Facilitators to eHealth Use in Daily Practice: Perspectives of Patients and Professionals in Dermatology. J Med Internet Res. 2017; 19: e300. 50. Cameron JI, Naglie G, Silver FL and Gignac MA. Stroke family caregivers' support needs change across the care

51. Wells S, Rozenblum R, Park A, Dunn M and Bates DW. Organizational strategies for promoting patien and provider uptake of personal health records. J Am Med Inform Assoc. 2015; 22: 213-22.

Li J, Talaei-Khoei A, Seale H, Ray P and Macintyre CR. Health Care Provider Adoption of eHealth. Systematic Literature Review. Interact J Med Res. 2013; 2: e7.

53. Ryan C, Bergin M and Wells JS. Theoretical Perspectives of Adherence to Web-Based Interventions: a Scoping Review. Int J Behav Med. 2018; 25: 17-29.

54. Michie S, Yardley L, West R, Patrick K and Greaves F. Developing and Evaluating Digital Interventions to Promote Behavior Change in Health and Health Care. Recommendations Resulting From an International Workshop. J Med Internet Res 2017: 19: e232.

55. Sumitani M. Miyauchi S, McCabe CS, et al. Mirror visual feedback alleviates deafferentation pain, depending on qualitative aspects of the pain: a preliminary report. Rheumatology (Oxford). 2008; 47: 1038-43.

56. Rothgangel A, Braun S, Winkens B, Beurskens A and Smeets R. Traditional and augmented reality mirro therapy for patients with chronic phantom limb pain (PACT study): results of a three-group, multicentre single-blind randomized controlled trial Clin Rehabil 2018: 32: 1591-608

57. Ortiz-Catalan M. Guethmundsdottir RA, Kristoffersen MB, et al. Phantom motor execution facilitated by (imb pain: a single group, clinical tria (2016; 388: 2885-94.

58. Osumi M. Inomata K. Inoue Y, Otake $Y$, Morioka S and Sumitani M. Characteristics of Phantom Limb Pain Alleviated with Virtual Reality Rehabilitation. Pain Med 2018; Dec 21

59. De Nunzio AM, Schweisfurth MA, Ge N, et al. Relieving phantom limb pain with multimodal sensorymotor training. J Neural Eng. 2018; 15:066022.
60. Rothgangel A and Bekrater-Bodmann R. Mirror therapy versus augmented/virtual reality applications:

61. Van Vels a tallored mechanism-based treatment for phantom limb pain. Pain Manag. 2019; 2: 151-159. portals for rehabilitation care: an exploratory focus group study with patients and healthcare professionals. BMC Med Inform Decis Mak. 2016; 16: 11.

62. Velsen $L V$, Tabak $M$ and Hermens H. Measuring patient trust in telemedicine services: Development of a survey instrument and its validation for an anticoagulation web-service. Int J Med Inform. 2017; 97: 52-8. Sieverink F, Kelders S, Poel M and van Gemert-Pijnen L. Opening the Black Box of Electronic Health-

Collecting, Analyzing, and Interpreting Log Data. JMIR Res Protoc. 2017; 6: e156.

64. Rothgangel A, Braun S, de Witte L, Beurskens A and Smeets R. Development of a Clinical Framework for

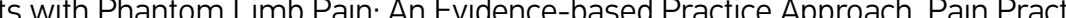
2016; 16: 422-34.

65. Herrador Colmenero L, Perez Marmol JM, Marti-Garcia C, et al. Effectiveness of mirror therapy, motor . Orthot Int. 2018: 42: 288-98.

66. Johnson MI Mulvey MR and Bognall AM. Transcutaneous electrical nerve stimulation (TENS) for phantom pain and stump pain following amputation in adults Cochrane Database Syst Rev 2015.8. CD007264.

67. Syst Rev. 2017; 5: CD011669.

68. Rothgangel AS, Braun SM, Beurskens AJ, Seitz RJ and Wade DT. The clinical aspects of mirror therapy in rehabilitation: a systematic review of the literature. Int J Rehabil Res. 2011; 34: 1-13.

Wentzel J, van der Vaart R, Bohlmeijer ET and van Gemert-Pijnen JE. Mixing Online and Face-to-Face . de Vries HJ Kloek CJJ, de Bakker DH. Dekker J Bossen D and Veenhof C. Determinants of Adhe to the Online Component of a Blended Intervention for Patients with Hip and/or Kne Osteor thrits A 
Moore MA, Coffman M, Jetty A, Petterson S and Bazemore A. Only 15\% of FPs Report Using Telehealth; Training and Lack of Reimbursement Are Top Barriers. Am Fam Physician. 2016; 93: 101.

Deutsche Rentenversicherung. Anforderungen an Tele-Reha Nachsorge 2018.

73. Nederlandse Zorgautoriteit. Wegwijzer bekostiging e-health. Utrecht 2018.

74. van Limburg M, van Gemert-Pijnen JE, Nijland N, Ossebaard HC, Hendrix RM and Seydel ER. Why business modeling is crucial in the development of eHealth technologies. J Med Internet Res. 2011; 13 ; e124.

75. van Limburg M, Wentzel J, Sanderman R and van Gemert-Pijnen L. Business Modeling to Implement an eHealth Portal for Infection Control: A Reflection on Co-Creation With Stakeholders. JMIR Res Protoc. 2015; 4: e104.

76. Chaet D, Clearfield R, Sabin JE, Skimming K, Council on E and Judicial Affairs American Medical A. Ethical practice in Telehealth and Telemedicine. J Gen Intern Med. 2017; 32: 1136-40,

2017,32: 1136-40. Terodicine Services and Information Technology. Med Arch. 2017; 71: 351-5.

78. Vayena E, Haeusermann T, Adjekum A and Blasimme A. Digital health: meeting the ethical and policy challenges. Swiss Med Wkly. 2018; 148: w14571.

79. US Department of Veterans Affairs. Million Veteran Program is Now Largest Genomic Database in the World. Washington DC 2016

80. Wojcicki A. Power of One Million. Mountain View: 23andMe, Inc., 2015 ,

81. Smalley E. Sanofi and Google in type 2 diabetes smartphone tie-up. Nat Biotechnol. 2016; 34: 1087-8.

82. Vayen E. Gasser U, Wood A O'Brien DR and Altman M. Elements of a New Ethical Framework for Big

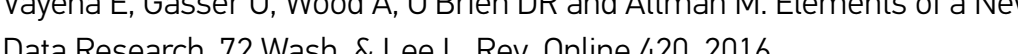

83. Townsend A, Leese J, Adam P, et al. eHealth, Participatory Medicine, and Ethical Care: A Focus Group Study of Patients' and Res. 2015; 17: e155.
84. Mort M, Roberts C, Pols J, Domenech M, Moser I and investigators E. Ethical implications of home Molecare for older people: a framework derived from a multisited participative study. Heal th Expect 2015 , 8: 438-49.

85. Kidholm K, Ekeland AG, Jensen LK, et al. A model for assessment of telemedicine applications: mast. In $J$ Technol Assess Health Care. 2012; 28: 44-51.

86. Kidholm K, Jensen LK, Kjolhede T, Nielsen E and Horup MB. Validity of the Model for Assessment of Telemedicine: A Delphi study. J Telemed Telecare. 2018; 24: 118-25. 


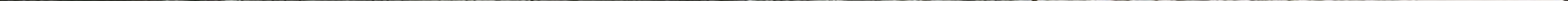


SUMMARY

Phantom limb pain following amputation is highly prevalent as it affects up to $80 \%$ of amputees. Many amputees suffer from phantom limb pain for many years and experience major limitations in daily routines and quality of life. Conventional pharmacological interventions offen have negative side-effects and evidence regarding their long-term efficacy is low. Central malplasticity such as the invasion of areas neighbouring the cortical representation of the amputated limb contributes to the occurrence and maintenance of phantom limb pain. In this context, alternative, non-pharmacological interventions such as mirror therapy that are thought to target these central mechanisms have gained increasing attention in the treatment of phantom limb pain. However, a standardized evidence-based treatment protocol for mirror therapy in patients with phantom limb pain is lacking, and evidence for its effectiveness is still low. Furthermore, given the chronic nature of phantom limb pain and suggested central malplasticity, published studies proposed hat patients should self-deliver mirror therapy over several weeks to month to achieve sustainable effects. To achieve this training intenstly, patients need to perform self-delivered exercises on lite is known about potential fenefts of using telerhabiltation in patients with phantom limb pin, and controlled clincel trials investigating

The present thesis presents the findings from the 'PAtient Centered Telerehabilitation' (PACT) project, which was conducted in three consecutive phases: 1) creating a theoretical foundation; 2) modelling the intervention; and 3) evaluating the intervention in clinical practice.

The objectives formulated for the three phases of the PACT project were:

1) to conduct a systematic review of the literature regarding important clinical aspects of mirror therapy. It focused on the evidence of applying mirror therapy in patients with stroke, complex regional pain syndrome and phantom limb pain.

2) to design and develop a clinical framework and a user-centred telerehabilitation for mirror therapy in patients with phantom limb pain following lower limb amputation 3) to evaluate the effects of the clinical framework for mirror therapy and the additional effects of the teletreatment in patients with phantom
limb pain. It also investigated whether the interventions were delivered by patients and therapists as intended.
Chapter 1 introduces the topic of this thesis. It describes the clinical relevance of phantom limb pain for rehabilitation and provides potential enance of this phenomenon. The chapter elaborates on non-pharmacological interventions including mirror therapy that address these neurophysiological mechanisms of phantom limb pain as an alternative to the standard medical treatment. It explores two important gaps in clinical practice and scientific research with regard to mirtor therapy. the inconsistency of how to deliver mirror therapy in clinical practice and the limited evidence for its effectiveness to reduce phantom limb pain. Chapter 1 also outhines hise relevance of using lechnology, e.g. telerehabilitation, to support and monitor self-delivered exercises to achieve sustainable effects. Subsequenty, ll presents the huge bartier that many novel telehealth applications face which a ex practice. At the end of Chapter 1 the aims of this thesis and an outline of the different phases related to these aims are presented.

At the start of the PACT project, little was known about important clinical aspects and the effects of mirror therapy in rehabilitation. Chapter 2 describes a systematic review of the literature regarding mirror therapy interventions after stroke, phantom limb pain and complex regional randomized trials were performed in stroke patients, and only two controlled studies with a total of 32 amputees with phantom limb pain were published. The trials were very heterogeneous regarding their design, the measures used as well as the intervention and patient characteristics, In general, the description of important clinical aspects for the delivery of mirror therapy in clinical practice was sparse. While individual studies suggested potential benefits of mirror therapy on phantom limb pain, the evidence was nonetheless low. Little was published about which patients are more likely to benefit from mirror therapy, but sufficient cognitive capacities seemed to be mandatory. Many different clinical methods of how to deliver mirror therapy were described, but detailed information and a standardized, evidence-based treatment protocol for mirror therapy was lacking. Only studies that used a mirror therapy intervention over several weeks reported effects.

Chapter 3 presents the development and content of a clinical framework for mirror therapy in patients with phantom limb pain. The development was based on an a-priori defined theoretical model, the different phases in methodological intervention defined by the Royal Dutch Society for Physical Therapy: informing the patient, history taking, physical examination, diagnosis, and indication for treatment, treatment (plan) and evaluation. Three sources of data collection were used to develop the clinical framework: first, we updated our systematic limb oan he through questionaires and semi-structured interviews. Thid, 
delivery of mirror therapy in clinical practice were also assessed through semi-structured interviews. The data from these three sources were clustered into main and subcategories and were used to complement and refine the theoretical model. Based on these categories, we developed a clinical flowchart and a comprehensive booklet that illustrates the individual phases of the clinical framework. The framework
includes important patient and intervention characteristics and can be used to personalize the delivery of mirror therapy in clinical practice.

Chapter 4 describes the user-centered approach that guided the design and development of the telerehabilitation platform for patients Win phan were identified through a questionnaire followed by a semi-structured interview. The second phase involved designing the interface of the west resulted in a medium-fidellity prototype whose usability was tested in routine care in the final third phase, leading to the development of a a structured decision matrix that incorporated the opinions of different disciplines such as the end-users, the research teap as well as

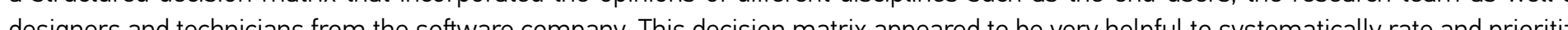
alluser requirements based on clear criteria The interprofessional participtory development approach and continuous, iterative evaluation throughout the development was very useful to develop a user-friendly high-fidelity prototype of the teletreatment

Chapter 5 describes the design of a three-arm multi-centre randomized controlled trial evaluating: 1) the effects of four weeks of traditional pain and 2) the additional effects of a six-week teletreatment after four weeks of traditional mirror therapy compared to self-delivered mirror therapy and self-delivered sensomotor exercises without a mirror. The primary and secondary outcome measures were chosen in correspondence with the and the guidelines from the Neuropathic Pain Special Interest Group (NeuPSIG). Chapter 5 further reflects on several questions concernin the study design that emerged during the preparation of the trial.

Chapter 6 reports the results of this randomized controlled trial, which included 75 lower limb amputees, regarding the effects of the traditional face-to-face and teletreatment approach to mirror therapy. We found limited effects of the traditional and the teletreatment approach Despite a careful and systematic design process of the interventions and a carefully designed trial, most of the differences between the experimental and control groups were neither statistically significant nor clinically worthwhile over all patients. Significant effects of mirror analysis suggested significant and clinically worthwhile effects of traditional mirror therapy in women, patients with telescoping and patien (t) framework for traditional mirror therapy was feasible in clinical practice. The teletreatment had no additional effects on phantom limb pain compared to self-delivered mirror therapy.

We performed a detailed process evaluation alongside the randomized controlled trial, which is reported in Chapter 7. The aims of this study were to assess 1) whether physical and occupational therapists delivered traditional mirror therapy according to the pre-defined clinical framework, 2) 1) whe and ten physical and occupational therapists participated in the process evaluation. Only sixteen patients (31\%) received traditional mirror therapy according to the clinical framework. The teletreatment was used by 14 patients (56\%) with suffient dose according to the protocol 政 showing higher acceptance rates and experiencing more benefits through the use of the teletreatment than therapists.

Finally, in Chapter 8, the main findings related to the different phases of the PACT project are discussed. Subsequently, several methodological aspects such as the choice of study design and the measures used are debated, followed by the lessons learned from the different phases of the project, which can be clustered into three topics. 1) the current evidence for mirtor therapy in patients with phantom limb pain; 2 ) the relevance of co-designing elleath together with different stakeholders; 3) the gap between theory and practice. In the last section, implications for research, clinical practice and education of future heath care professionals are described. The implications for research are related to various aspects better match the principle of personalized care and the complex and disruptive nature of eHealth interventions. The implications for clinical practice concem, amongst ohers, prerequisiles for fur her implent or healh care professionals and pro the potential advantages, limitations and failures of using eHealth in daily care. 


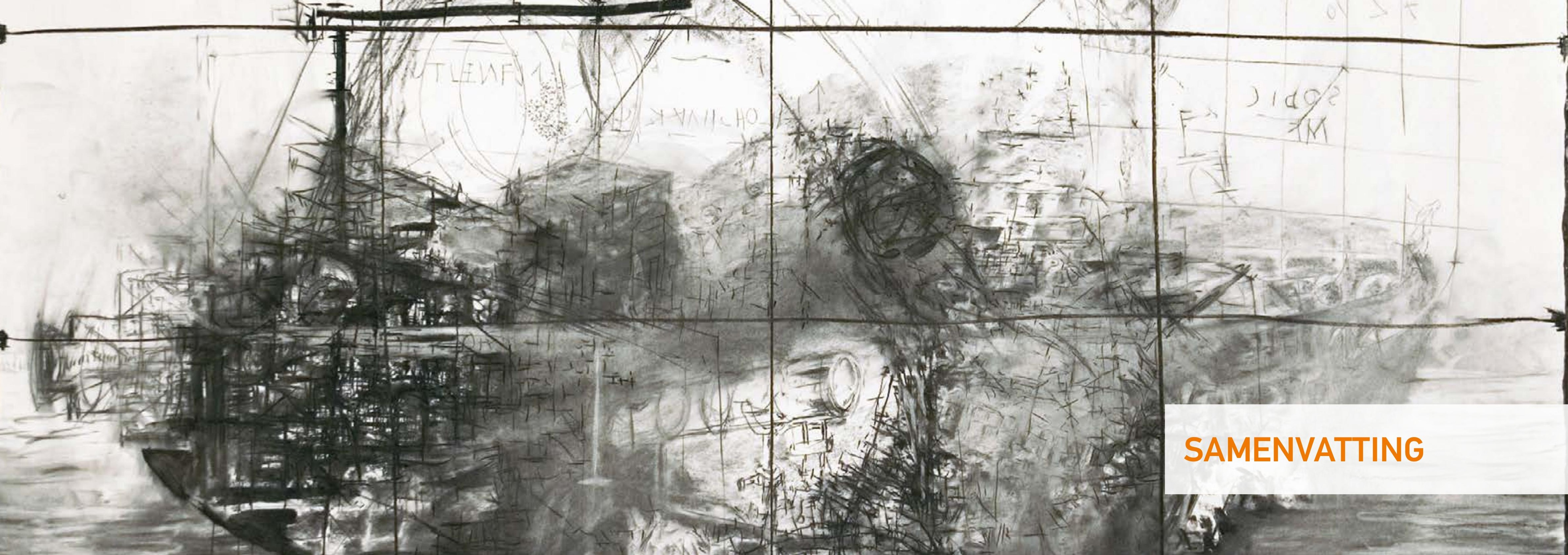


(is fantoompijn. Uit onderzoek blijkt dat tot $80 \%$ van de geamputeerde patiënten hier last van heeft. Veel patiënten lijden jaren aan fantoompijn en ervaren grote beperkingen in hun dagelijks leven. (negatieve) bijwerkingen en het bensijs voenties voor de kwaliteit van hun leven. Conventionele farmacologische interventies hebben vaak gebieden in de hersenen draagt bij aan het ontstaan en in stand houden van fantoompiin. Door sensitisatie van neuronen en gliacellen in het corticale gebied van de geamputeerde extremiteit reageren deze cellen op activiteit van het aangrenzende gebieden en kunnen prikkels uit ( richten hebben de afgelopen jaren steeds meer aandacht gekregen in de behandeling van fantoompiin. Een voorbeeld van een dergelijke

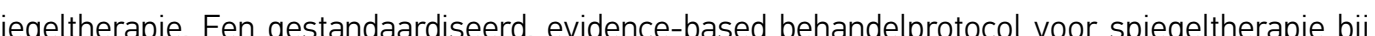
patiënten met fantoompiin ontbreekt momenteel echter, en het bewijs voor het effect van deze therapie op fantoompiin is nog steeds beperkt. Bovendien blijkt uit gepubliceerde studies, dat patiënten gezien de chronische aard van fantoompiin en de veronderstelde centrale Om deze training zat en gecontroleerd klinisch effectonderzoek ontbreekt.

In dit proefschrift worden de resultaten van het ,PAtient Centered Telerehabilitation' (PACT) project beschreven. Het PACT project werd en 3) evaluatie van de interventie in de klinische praktijk. De doelstellingen van deze proefschrift hebben dan ook betrekking op deze drie fasen:

De doelstelling van de eerste fase was het uitvoeren van een systematische review van de literatuur met betrekking tot belangrijke met een beroerte, complex regionaal pijnsyndroom en fantoompiin in kaart gebracht (Hoofds Uk 2). Det valu het project zijn vervolgens gebruikt als input voor de tweede fase. Hierin stond het ontwerpen en ontwikkelen van een klinisch raamwerk en een gebruiker-gecentreerde telerevalidatie platform op basis van spiegeltherapie voor patienten met fantoompinn na een amputatie van de onderste extremiteit centraal (Hoofdstukken 3-4). Resultaten uit deze fase zijn gebruikt om een driearmige effectstudie met betrekking tot het klinische raamwerk en de telerevalidatie platform vorm te geven (Hoofdstuk 5). In de derde fase zijn de effecten van het klinische raamwerk voor spiegeltherapie en de additionele effecten van de telerevalidatie bij patiënten met fantoompiin in de klinische praktijk geëvalueerd. Tot slot werd onderzocht of de interventies door patiënten en therapeuten zinn uitgevoerd zoals bedoeld (Hoofdstukken 6-7).

In Hoofdstuk 1 wordt het onderwerp van dit proefschrift geïntroduceerd. De klinische relevantie van fantoompinn wordt beschreven en potentiële neurofysiologische mechanismen zoals corticale reorganisatie die bijdragen aan het ontstaan en handhaven van dit fenomeen worden gepresenteerd. Er wordt ingegaan op niet-farmacologische interventies, waaronder spiegeltherapie, die deze neurofysiologische mechanismen als een alternatief voor de standaard medische behandeling adresseren. Vervolgens wordt in het hoofdstuk ingegan op twee belangrijke lacunes in de klinische praktijk en wetenschappelijk onderzoek met betrekking tot spiegeltherapie: de inconsistente uitvoering van spiegettherapie door professionals in de klinische praktijk en het beperkte bewijs voor de effectiviteit van spiegeltherapie ten aanzien van het verminderen van fantoompijn. Bovendien wordt in het hoofdstuk de relevantie van het gebruik van telerevalidatie geschetst om zelfstandig ofenen van de patïnt te ondersteunen. Vervolgens wordt de enorme barrière gepresenteerd warme veel nieuwe eHealth toepassingen worden geconfronteerd. onvoldoende acceptatie door de eindgebruikers in de klinische praktijk Onvoldoende betrokkenheid van de eindgabruikers blikt hierbij een belangrijke oorzakelike rol te spelen. De inleiding eindigt met een beschriving van de doelstellingen en structuur van dit proefschrift.

Bij de start van het PACT project was er weinig bekend over belangrijke klinische aspecten en de effecten van spiegeltherapie in de revalidatie. (

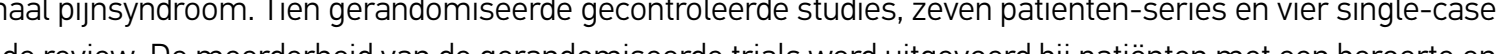
slechts twee gecontroleerde studies werden gepubliceerd over spiegeltheranie bij fantoompiin met in total 32 patïnten De trials waren zeer heterogeen met betrekking tot hun design, de gebruikte meetinstrumenten evenals de karakteristieken van de interventie en geïncludeerde

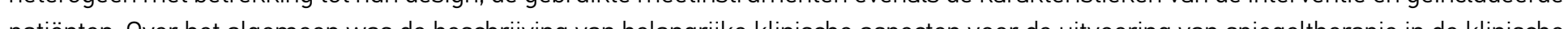

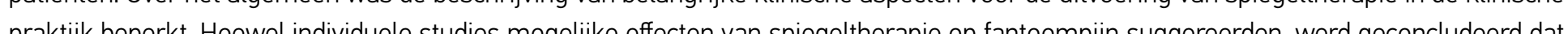


de mate van bewijs laag is. Alleen studies waarin spiegeltherapie over meerdere weken werd uitgevoerd rapporteerden effecten

Er werd weinig informatie gevonden over welke patiënten meer kans op positieve effecten door spiegeltherapie hebben, maar het hebben van voldoende cognitieve capaciteiten leek een belangrijke voorwaarde te zijn. Er werden veel verschillende manieren beschreven hoe spiegeltherapie werd utgevoerd maar gedetailleerdere informatie en een evidence-based behandelprotocol voor spiegeltherape ontbraken.

Hoofdstuk 3 presenteert de ontwikkeling en opbouw van een klinisch raamwerk voor spiegeltherapie bij patiënten met fantoompijn. De opbouw is gebaseerd op de verschillende fasen van het fysiotherapeutisch methodisch handelen, gedefinieerd door de Koninklijke Nederlandse Genootschap voor Fysiotherapie: Informeren van de patiënt, screening en afnemen van de anamnese, lichamelijk onderzoek, diagnose en indicatie voor behandeling, behandel(plan) en evaluatie. Drie bronnen van gegevensverzameling werden gebruikt om het klinische raamwerk te ontwikkelen: Ten eerste werd de in hoofdstuk 2 beschreven systematische review van de literatuur met betrekking tot belangrijke klinische aspecten en het effect van spiegeltherapie bij patïnten met fantoompiin geüpdatet. Daarnaast werden klinische ervaringen van fysio- en ergaherapeuten die patienten met fantoompiin behandelden geanalyseerd met behulp van vragenlijsten en semi-gestructureerde interviews. Ten derde werden de voorkeuren van patiënten met fantoompijn met betrekking tot de uitvoering van spiegeltherapie in de klinische praktijk in kaart gebracht met behulp van semi-gestructureerde interviews. De gegevens van deze drie bronnen werd Op basis van deze categorieän werd een klinisch stroomdiagram en een uitgebreide handleiding ontwikkeld die de verschillende fasen van het klinische rammwerkillustreren Het rammerk bevat belangrijke patïnt- en interventiekarkteristieken en kan worden gebruikt om de uitvoering van spiegettherapie in de klinische praktijk op maat aan te bieden

Hoofdstuk 4 beschrijtt de gebruiker-gecentreerde aanpak voor het ontwerp en de ontwikkeling van het telerevalidatie platform voor patiënten met fantoompiin in

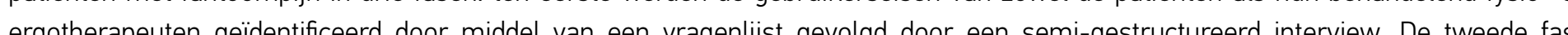
bestond wit het ontwerpen van de interface van de telerevalidatie met behulp van schetsen, wireframes en interface mock ups om en low-fidelity prototype te ontwikkelen. Heuristische evaluatie resulteerde in een medium-fidelity prototype waarvan in de derde fase de de dagelijkse praktijk om tot een high-fidelity prototype van het telerevalidatie platform te kom
Om de gebruikerseisen te prioriteren, was het noodzakelijk om een gestructureerde beslissingsmatrix te ontwikkelen en toe te passen waarin de meningen van verschillende disciplines zoals de eindgebruikers, het onderzoeksteam, ontwerpers en programmeurs waren verwerkt. Deze beslissingsmatrix bleek erg nuttig om systematisch op basis van verschillende criteria alle gebruikerseisen te beoordelen en te prioriteren. Bovendien was de interprofessionele, participatieve aanpak en de continue, iteratieve evaluatie gedurende de ontwikkeling erg nuttig om een gebruiksvriendelijke high-fidelity prototype van de telerevalidatie platform te ontwikkelen.

Hoofdstuk 5 beschrijtt het design van een 'multi-center randomized controlled trial' waarbij de deelnemers naar een van de drie groepen werden gerandomiseerd. Het doel van deze studie was, om: 1) de effecten van vier weken spiegettherapie volgens het klinische raamwerk in vergelijking met sensomotorische oefeningen zonder spiegel op fantoompijn te evalueren en 2) de aanvullende effecten van zes weken telerevalidatie aansluitend aan de vier weken spiegeltherapie in vergelijking met zelfstandig uitgevoerde spiegeltherapie en zelfstandig uitgevoerde sensomotorische oefeningen zonder spiegel te onderzoeken. De primaire en secundaire uitkomstmaten werden conform de aanbevelingen van het Initiative on Methods. Measurements, and Pain Assessment in Clinical Trials (IMMPACT) en de richtlinen van de Neuropathic Pain Special Interest Group (NeuPSIG) gekozen Hoofdsth 5 studiedesign die in de voorbereidende fase van de gerandomiseerde, gecontroleerde studie naar voren kwamen.

Hoofdstuk 6 rapporteert de resultaten van deze gerandomiseerde en gecontroleerde studie, waarin de face-to-face spiegeltherapie en de telerevaldatie platform met sensomotorische oefeningen zonder spiegel werden vergeleken In de studie werden 75 patiên 政 vooruitgang zien Ondanks een zorgvuldig en systematisch ontwerpproces van de interventies en het design van de trial waren de meeste verschillen tussen de experimentele en controlegroepen niet statistisch signifcant noch klinisch relevant Signifcante effecten werden alleen in de per-protoco-analyse, dus in petienten die ten minste 10 sessies spiegetherape gedurende 4 weken ontvingen, gedetecteerd Bovendien liet de subgroep analyse signifcante en klinisch re fenomeen en paï̈nten die een motorische componentin relatie methet type fantoompin warnemen (bv. krampend gevoel). De evalue toonde verder aan dat het klinische raamwerk voor spiegeltherapie hanteerbaar was in de klinische praktijk.

Parallel aan de effectstudie werd een gedetailleerde procesevaluatie uitgevoerd, die in hoofdstuk 7 wordt beschreven. De vraagstellingen 
van deze studie waren 1) of fysio en ergotherapeuten spiegeltherapie volgens het vooraf gedefinieerde klinische raamwerk uitgevoerd hebben; 2) welke oefenprogramma's van de telerevalidatie platform door patiënten werden gebruikt en in welke mate, en 3) hoe hoog de acceptatie onder patiënten en therapeuten met betrekking tot het gebruik van de telerevalidatie platform was en welke ervaringen zij met het platform hadden opgedaan. Eenenvijftig patiënten met fantoompijn en 10 fysio- en ergotherapeuten namen deel aan de procesevaluatie. Slechts 16 patiënten (31\%) werden volgens het klinische raamwerk met spiegeltherapie behandeld in de eerste 4 weken van de studie. Van de patiënten die in de telerevalidatie groep werden ingedeeld, werd de telerevalidatie platform door slechts 14 van de 26 patiënten (56\%) volgens het protocol gebruikt. Potentiële barrières voor de implementatie van de telerevalidatie platform die gebruikers ervoeren waren gerelateerd aan onvoldoende training vooraf en gebrek aan support tijdens het gebruik van het platform. Daarnaast bleken technische problemen vaak voor te komen waardoor de gebruiksvriendelijkheid negatief werd beïnvloed. De acceptatie van de telerevalidatie platform door patiënten en therapeuten was voldoende warbij patiënten een hogere mate van acceptatie aangaven. Patiënten zagen ook meer specifeke voordelen door het cabruik van de telerevalidatie platform (bv om hun fantoompiin te controleren) dan therapeuten. Theraperten 列 klinische routines te implementeren. Ten slotte worden in hoofdstuk 8 de belangrijkste bevindingen met betrekking tot de verschillende fasen van het PACT project gepresenteerd gebruikte uitkomstmaten, gevolgd door de "lessons learned" uit de verschillende fasen van het project. Deze 'lessons learned" kunnen geclusterd worden in drie topics. 1) het huidige bewis voor spiegeltherapie bij patienten met fontoompiin 2) de relevantie om eHeath samen met verschillende stak: 1) hers in co-creatie te ontwikkelen 3) de klof tussen theorie en praktijk In het latste deel worden de implicaties voor onderzoek, klinische praktijk en onderwiis van (toekomstige) professionals in de gezondheidszorg beschreven. De implicaties voor onderzoek he ben betrekking op verschillende aspecten, zoals hetidentificeren van 'responders' om een gepersonaliseerde behandeling te faciliteren. Daarnaast wordt ingegaan op het gebruik van alternatieve onderzoekdesigns die mogelijk beter ans bii het principe van gepersonliseerde zorg en de cophlexe en disuptieve ard van etralth. Traditionele onderzoeksdesigns zoals gerandomiseerde, gecontroleerde experimenten (RCTs) zijn minder goed geschikt om de effecten van interventies die nog niet goed in de praktijk aemplementerd zijn zoals ethelth te evalueren. De implicaties voor de klinische praktijk betreffen onder meer de vorwarden voor verdere implementatie van spiegeltherapie en de telerevalidatie phatorm in de dagelijks praktijk en belangijke ethishe aspecten zoals dataprivacy en bescherming van persoonlijke data die moeten worden overwogen. Ten slotte wijzen de implicaties voor het onderw op het belang van bewustwording bij studenten fysiotherapie en ergotherapie voor mogelijke niet-medicamenteuze interventies in de beperkingen en mislukkingen van het gebruik van eHealth in de dagelijkse praktijk. 
tints

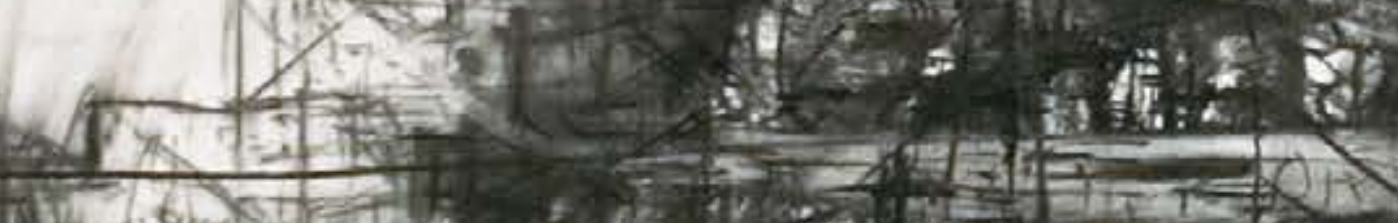
Hen

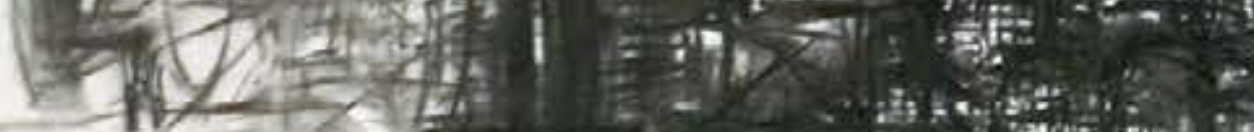
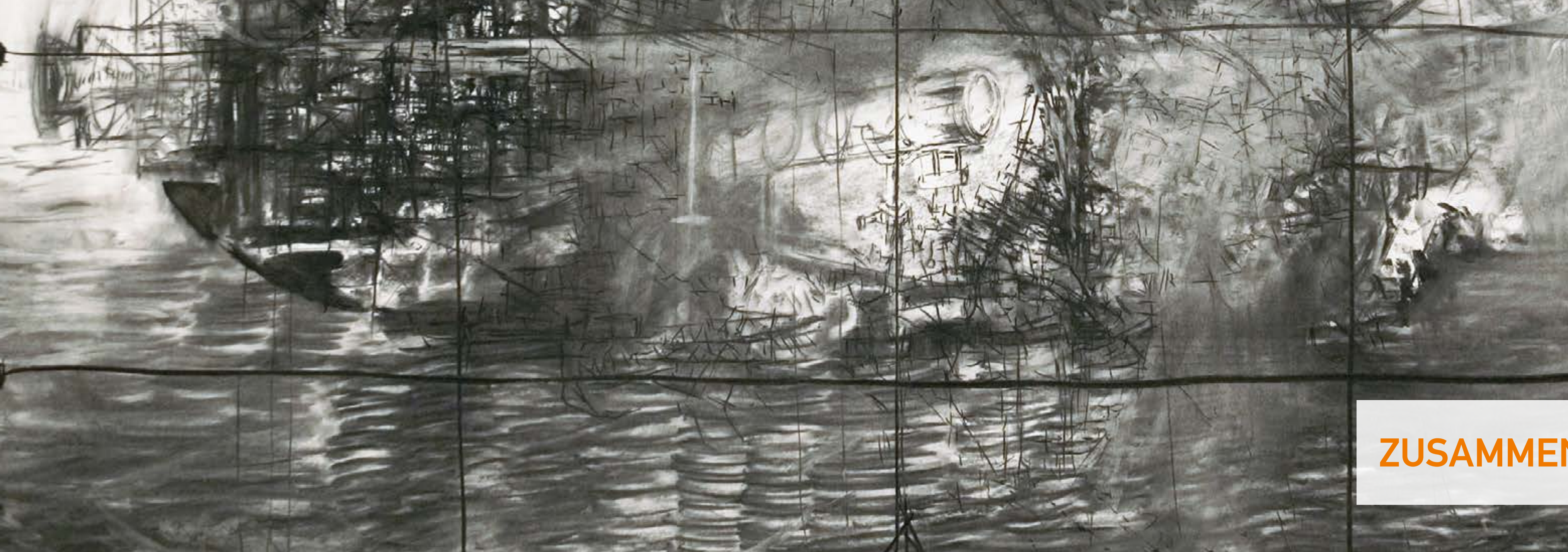

at)

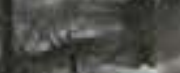

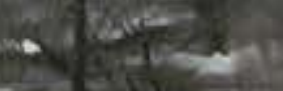

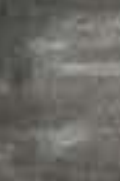

\section{ZUSAMMENFASSUNG}

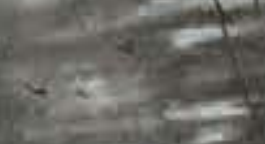




\section{ZUSAMMENFASSUNG}

Phantomschmerzschmerzen nach Amputationen stellen ein klinisch häufig vorkommendes Problem dar, da bis zu 80\% der Amputierten hiervon betroffen sind Ein Großteil der Amputierten leidet viele Jahre an Phantomschmerzen und empfindet hierdurch erhebliche Einschränkungen im Altag und der Lebensqualitat. Konventionelle pharmakologische Behandlungen sind häufig mit deutlichen Nebenwirkungen verbunden, und die Evidenz für nachhaltige Effekte auf den Phantomschmerz ist gering. Zentrale Malplastizität, wie der Prozess der kortikalen Reorganisation von differenzierten Hirnarealen, trägt zum Auffreten und der Aufrechterhaltung von Phantomschmerzen bei. In diesem Kontext haben alternative, nicht-medikamentöse Verfahren wie die Spiegettherapie, die diese zentralen Mechanismen adressieren, zunehmende Aufmerksamkeit erlangt. Es fehlt bislang jedoch ein standardisiertes, evidenzbasiertes Behandlungsprotokoll fur die Spiegelherapie, und die Evidenz fur ihre Wirksamkelt bei Paltentinnen und Pallenten mit Phantomschmerzen ist nach wie vor gering. In Anbetracht der Chronizilat der Phantomschmerzen und der zentralen maladaptiven Prozesse, emprehlen publizierte Studien die Spiegeltherapie regelmaßig uber einen Zeitraum von mehreren Wochen bis Monaten durchzufuhren, um nachhaltige Effekte zu erzielen. Um diese Trainingsintensität zu erreichen, mussen die Betroffenen regelmäßig eigenständige Ubungen im häuslichen Umfeld absolvieren. Hierbei treten jedoch haüfig Fragen und Unsicherheiten seitens der Patientinnen und Patienten auf und werden die eigenständigen Ubungen meist nicht mit der erforderlichen Intensilat genuzz. Das eigenstandige Training kann durch den Ensalz von Informations- und Telerabailitation bei Patientinnen und $P_{a}$ Studien zur Evaluation klinischer Effekte fehlen.

Die vorliegende Arbeit präsentiert die Ergebnisse des Projekts, PAtient Centered Telerehabilitation' (PACT), das in drei aufeinander folgenden Phasen durchgeführt wurde: 1) Entwicklung eines theoretischen Fundaments; 2) Modellierung der Intervention; und 3) Evaluation der

Die für die drei Phasen des PACT Projekts formulierten Ziele lauteten:

1) Eine systematische Literaturrecherche zu wichtigen klinischen Aspekten der Spiegeltherapie durchzuführen. Der Fokus der Recherche lag auf der Evidenz für die Anwendung der Spiegeltherapie bei Patientinnen und Patienten mit Schlaganfall, komplexem regionalem Schmerzsyndrom und Phantomschmerz.
2) Konzeption und Entwicklung eines klinischen Behandlungsleitfadens und einer nutzerzentrierten Telerehabilitationsplattform für die Spiegetherapie bei Patientinnen und Patienten mit Phantomschmerzen nach einer Amputation der unteren Extremität.

3) Evaluation der klinischen Effekte des Behandlungsleitfadens für die Spiegeltherapie und des zusätzlichen Nutzens der Telerehabilitation bei Patientinnen und Patienten mit Phantomschmerzen. Darüber hinaus wurde innerhalb einer Prozessevaluation untersucht, ob die Interventionen wie geplant von den Betroffenen und den behandelnden Therapeutinnen und Therapeuten durchgeführt wurden.

Kapitel 1 führt die Leserinnen und Leser in die Thematik dieser Arbeit ein. Es beschreibt die klinische Relevanz von Phantomschmerzen und Aufrechterhaltung dieses Schmerzsyndroms beitragen. Das Kapitel geht danach näher auf nicht-medikamentöse Verfahren wie die Spiegeltherapie ein, die diese neurophysiologischen Mechanismen als Alternative zur medikamentösen Behandlung adressieren. Im Anschluss werden zwei wichtige Lücken in Bezug auf den Versorgungsalltag und die wissenschaftliche Forschung zur Spiegeltherapie aufgezeigt: die inkonsistente Durchführung der Spiegettherapie in der klinischen Praxis und die begrenzte Evidenz für ihre Wirksamkeit zur Reduktion von Phantomschmerzen. In Kapitel 1 wird darüber hinaus die Relevanz von Digital-Health-Anwendungen wie Telerehabilitation zur Förderung des Eigentrainings und der nachhaltigen Sicherung klinischer Effekte beschrieben. Anschließend werden mögliche Hürden für die erfolgreiche Implementierung neuartiger Digital-Health-Anwendungen in den Versorgungsalltag präsentiert: Fehlende Nutzerakzeptanz und Integration in bestehende klinische Prozesse. Am Ende von Kapitel 1 werden die Ziele des PACT-Projekts und ein Überblick über die verschiedenen Projektphasen, die diesen Zielen zugrunde liegen, vorgestellt.

Zu Beginn des PACT Projekts existierten wenig Erkenntnisse auf Basis wissenschaftlicher Studien zu wichtigen klinischen Aspekten und möglichen Effekten der Spiegeltherapie innerhalb der Rehabilitation verschiedener Patientengruppen. Kapitel $\mathbf{2}$ beschreibt eine systematische Literaturrecherche hinsichtlich der Spiegeltherapie bei Patientinnen und Patienten nach Schlaganfall, komplexem regionalem Schmerzsyndrom und Phantomschmerzen nach Amputationen. Zehn randomisierte kontrollierte Studien, sieben Fallserien und vier Einzelfallstudien wurden in die Analyse einbezogen. Die Mehrzahl der randomisierten Studien wurde an Patientinnen und Patienten nach Schlaganfall durchgeführt, und lediglich zwei kontrollierte Studien an insgesamt 32 Patientinnen und Patienten mit Phantomschmerzen nach Amputationen wurden verofenticht Die Studien waren hinsichtlich ihres Designs, der verwendeten Messinstrumente, sowie der 
Interventions- und Populationsmerkmale sehr heterogen. Im Allgemeinen wurden in den analysierten Studien wichtige klinische Aspekte für die Durchführung der Spiegeltherapie im Versorgungsalltag nur sehr begrenzt beschrieben. Während einzelne Studien mögliche Effekte der Spiegeltherapie auf den Phantomschmerz aufzeigten, war die Evidenz insgesamt jedoch gering. Darüber hinaus konnte durch die Analyse der vorliegenden Studien keine eindeutige Schlussfolgerung gezogen werden, welche Patientinnen und Patienten eher von der Spiegeltherapie profitieren, aber ausreichende kognitive Fähigkeiten schienen obligatorisch zu sein. In den Veröffentlichungen wurden unterschiedliche klinische Methoden zur Durchführung der Spiegeltherapie beschrieben, allerdings fehlten ein standardisiertes, evidenzbasiertes Behandlungsprotokoll und detailliertere Informationen zur genauen klinischen Vorgehensweise. Lediglich Studien, in denen die Spiegeltherapie über mehrere Wochen hinweg durchgeführt wurde, berichteten signifikante Effekte.

Kapitel 3 präsentiert die Entwicklung und den Aufbau eines klinischen Behandlungsleitfadens für die Durchführung der Spiegettherapie bei Patientinnen und Patienten mit Phantomschmerzen. Die Entwicklung basierte auf einem zuvor definierten theoretischen Model den verschiedenen Phasen des physiotherapeutisch-methodischen Handelns, die von der Königlich Niederländischen Gesellschaft für Physiotherapie (KNGF) defniert wurden Aufklärung des Patienten. Anamnese, körperliche Untersuchung Diagnose und Indikationstellung Behandlungs(plan) und Evaluation. Innerhalb des Entwicklungsprozesses des klinischen Behandlungsleitadens wurden drei Datenquelle verwendet: Zuerst wurde de in Kapitel 2 beschriebene systematische Literaturübersicht zu wichtigen klinischen Aspekten und der Evidenz für die Wirksamkeit der Spiegltherapie bei Patientinnen und Patienten mit Phantomschmerzen aktualisiert Daüber himaus wurden de klinischen Erfahrungen von Physio- und Ergotherapeut innen, die Betroffene mit Phantomschmerzen behandelten, mittels Fragebägen und semi-strukturierten Interviews analysiert Z Zuetzzt wurden die Präferenzen von Patientinnen und Patienten die an Phantomschmerzen leiden hinsichtlich der praktischen Durchführung der Spiegeltherapie durch semi-strukturierte Interviews erfasst. Die Daten aus diesen drei Quellen wurden in Haupt- und Unterkategorien zusammengefasst und zur Ergänzung und Verfeinerung des theoretischen Modells verwendet Basierend auf diesen Kategorien entstanden ein Klinisches Flussdigramm und eine umfassende Broschüre, die die einzel Phasen des Klinischen Behandlungsleiffadens veranschaulichen. Der Behandlungsleitfaden enthält klinisch relevante PopulationsInterventionsmerkmale und kann genutzt werden um die Durchführung der Spiegeltherapie im Versorgungsalltag zu personalisieren.

Kapitel 4 beschreibt den nutzerzentrierten Entwicklungsprozess für die Konzeption und Entwicklung der Telerehabilitationsplattform der Plattform sowohl bei Patientinnen und Patienten mit Phantomschmerzen als auch Physio- und Ergotherapeut_innen anhand eines Fragebogens und semi-strukturierten Interviews durchgefuhrt. In der zweiten Phase wurde in Ko-Kreation mit Patientinnen und Patienten sowie Therapeutinnen und Therapeuten auf Basis des Anforderungskatalogs unter Verwendung von Entwurfsskizzen, Mockup's und graphischen Entwurfsvorlagen ein erster Prototyp mit niedriger Wiedergabetreue der Telerehabilitationsplattform entwickel. Darauffolgend wurde die Benutzerfreundlichkeit unter standardisierten Bedingungen durch heuristische Evaluation überprüutt, um einem Prototyp mit mittlerer Wiedergabetreue zu gelangen. In der abschließenden dritten Phase wurden dessen Benutzerfreundlichke und Funktionsfähigkeit von Patientinnen und Patienten sowie Therapeutinnen und Therapeuten im Versorgungsalltag evaluiert, woraufhin ein Prototyp mit hoher Wiedergabetreue entwickelt wurde. Innerhalb des Entwicklungsprozesses der Telerehabilitationsplattform war es notwendig eine strukturierte Entscheidungsmatrix zu entwickeln und anzuwenden, um die Anforderungen der Nutzerinnen und Nutzer zu priorisieren. Die Matrix integriert die Meinungen und Sichtweisen wichtiger Stakeholder innerhalb eines Digital-Health-Projekts wie der Endnutzer des Forschungsteams sowie des Softwareentwicklers. Diese Entscheidungsmatix war sehr hilfeich, um alle Anforderungen anhand klarer Kriterien systematisch zu bewerten und zu priorisieren. Darüber hinaus erwiesen sich der interprofessionelle, pritizipative Entwicklungsansatz sowie die kontinuierliche, iterative Evaluation während des gesamten Entwicklungsprozesses als essentiell, um eine benutzer_innenfreundliche Telerehabilitationsplattform zu entwickeln.

Kapitel 5 beschreibt das Design einer dreiarmigen, multizentrischen randomisierten, kontrollierten Studie, in der folgende Aspekte evaluie wurden.1) Die Effekte einer vierwöchigen ST geltherape (a) sechswöchigenT Tereh bilitation im Anschluss an die vierwöchige klassische Siegeltherapie im Vergleich zu sechswöchigen Heimübunger mit klassischer Spiegltherapie oder sensomotorischen Heimübungen des intakten Beins ohne Spiegel. Die primären und sekundäre Endpunkte wurden in Übereinstimmung mit den Empfehlungen der 'Initiative on Methods, Measurements, and Pain Assessment in Clinica

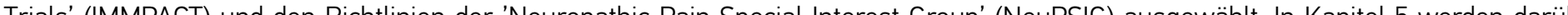
hinaus einige Überlegungen hinsichtlich des Studiendesigns diskutiert, die während der Planung der Studie auftraten.

Kapitel 6 berichtet die Ergebnisse der randomisierten, kontrollierten Studie zu den Effekten der klassischen Spiegeltherapie und der 
kleine Effekte der klassischen Spiegeltherapie im Vergleich zu sensomotorischen Übungen ohne Spiegel im Versorgungsalltag. Über den Interventionszeitraum verbesserten sich alle Studiengruppen hinsichtlich der meisten primären und sekundären Endpunkte. Trotz einer sorgältigen und systematischen Studienplanung und Entwicklung der Interventionen erwiesen sich die meisten Unterschiede zwischen den experimentellen Gruppen und der Kontrollgruppe über alle Patientinnen und Patienten betrachtet weder als statistisch signifikant noch klinisch relevant. Signifikante Effekte der klassischen Spiegeltherapie wurden lediglich in der ,Per-Protokoll-Analyse' festgestellt, d. h. bei Patientinnen und Patienten, bei denen mindestens 10 Behandlungseinheiten über 4 Wochen durchgeführt wurden. Darüber hinaus deutete die Subgruppenanalyse auf signifikante und klinisch relevante Effekte der klassischen Spiegeltherapie insbesondere bei Frauen und Patientinnen und Patienten mit Teleskopphänomen hin, sowie bei Patientinnen und Patienten, die eine motorische Komponente innerhalb der Phantomschmerzempfindung wahrnahmen (z. B. krampfartiges Gefühl). Die Ergebnisse zeigten weiter, dass der klinische Behandlungsleitfaden für die klassische Spiegeltherapie im Versorgungsalltag anwendbar ist. Die Nutzung der Telerehabilitationsplattorm im Vergleich zu eigenstandigen Lbungen mit der klassischen Spiegetherapie zeigte keine zustzlichen Effekte auf die Phantomschmerzen.

Parallel zur randomisierten kontrollierten Studie wurde eine detaillierte Prozessevaluation durchgeführt, die in Kapitel 7 näher beschrieben wird. Innerhalb dieser Studie wurde untersucht 1) ob Physio- und Ergotherapeut innen die klassische Spiegeltherapie gemä 8 dem klinischen Behandlungsleiffaden durchgeführthaben 2) welche Ubungsprogrammeder Telerehabilitationsplattform von den Patientinnen und Patienten genutzt wurden und in welchem Umfong, und 3) wie hoch die Akzeptanz unter Patientinnen und Patienten sowie Therapeutinnen und Therapeutenbez̈̈glich derTelerehabilitation warundwelcheErfahrungendie Nutzer innenim UmgangmitderPlattformimVersorgungsaltag machten. Insgesomt naten 51 Patientinnen und Patenten mit Phantomschmer der Prozessevaluation teil Lediglich 16 Patientinnen und Patienten (31\%) erhielen die klassische Spiegetherapie gemäß dem klinischen Behandlungsleitaden. Nach den ersten 4 Wochen Klassischer Spl (3\%) Patientinnen und Patienten (56\%) mit ausreichender Intenstät genutzt. Die Umsetzungshürden die von den Nutzerinnen und Nutzern im Zusammenhang mit der Telerehabiltation wahrgenommen wurden, bezogen sich auf unzureichende Schulung und Unterstützung bei der Nuzung der Plattorm some die Härfikeit technischer Probleme Dennoch zeigten sich zufriedenstellende Akzeptanzreten unter den Nezerinnen Und Nuzern der Telerehabiltation, wobei Patientinnen und Patienten etwas hähere Akzeptanzraten aufwiesen und einen ( in Bezug auf ihren Versorgungsalltag.
Abschließend werden in Kapitel 8 zuerst die wichtigsten Ergebnisse in Bezug auf die verschiedenen Phasen des PACT Projekts diskutiert. Im Anschluss werden mehrere methodische Aspekte wie die Wahl des Studiendesigns und die verwendeten primaren und sekundären Endpunkte diskutiert, gefolgt von den Lehren die aus den verschiedenen Projektphasen gezogen werden konnen und die sich in drei Themenbereiche gliedern lassen: 1) die momentane Evidenz für die Wirksamkeit der Spiegeltherapie bei Patientinnen und Patienten mit Phantomschmerzen; 2) die Relevanz einer nutzer innnenzentrierten Entwicklung von Digital-Health-Anwendungen unter Einbezug verschiedener Stakeholder_innen; 3 ) die Diskrepanz zwischen Theorie und Versorgungsalltag. Im letzten Abschnitt werden die Implikationen der Ergebnisse der vorliegenden Arbeit für die Forschung, den Versorgungsalltag sowie die Aus- und Weiterbildung (zukünftiger) Physio- und Ergotherapeut innen beschrieben. Die Implikationen für die Forschung beziehen sich auf unterschiedliche Aspekte, wie der Identifikation von Respondern für die Spiegeltherapie um eine personalisierte, maßgeschneiderte Behandlung zu ermöglichen. Darüber hinaus werden alternative Forschungsdesigns diskutiert, die besser geeignet sind um den Impact von personalisierten Behandlungsprogrammen sowie komplexer Digital-Health-Anwendungen zu evaluieren. Die Implikationen für den Versorgungsalltag beziehen sich unter anderem auf Voraussetzungen für die weitere Implementierung der Telerehabilitation in den Versorgungsalltag sowie wichtige ethische Aspekte, wie ausreichender Datenschutz und Transparenz die hierbei berücksichtigt werden sollten. Die Implikationen für die Aus- und Weiterbildung (zuküntiger) Physio- und Erotheraneut innen verweisen auf die Relevanz ein größeres Bewusstsein für nicht-medikamentöse Behandlungsmöglichkeiten für Patientinnen und Patienten mit Phantomschmerzen zu schaffen sowie auf die Voraussetzungen, Vorteile und Grenzen der Nutzung von Digital-Health-Anwendungen im Versorgungsalltag. 


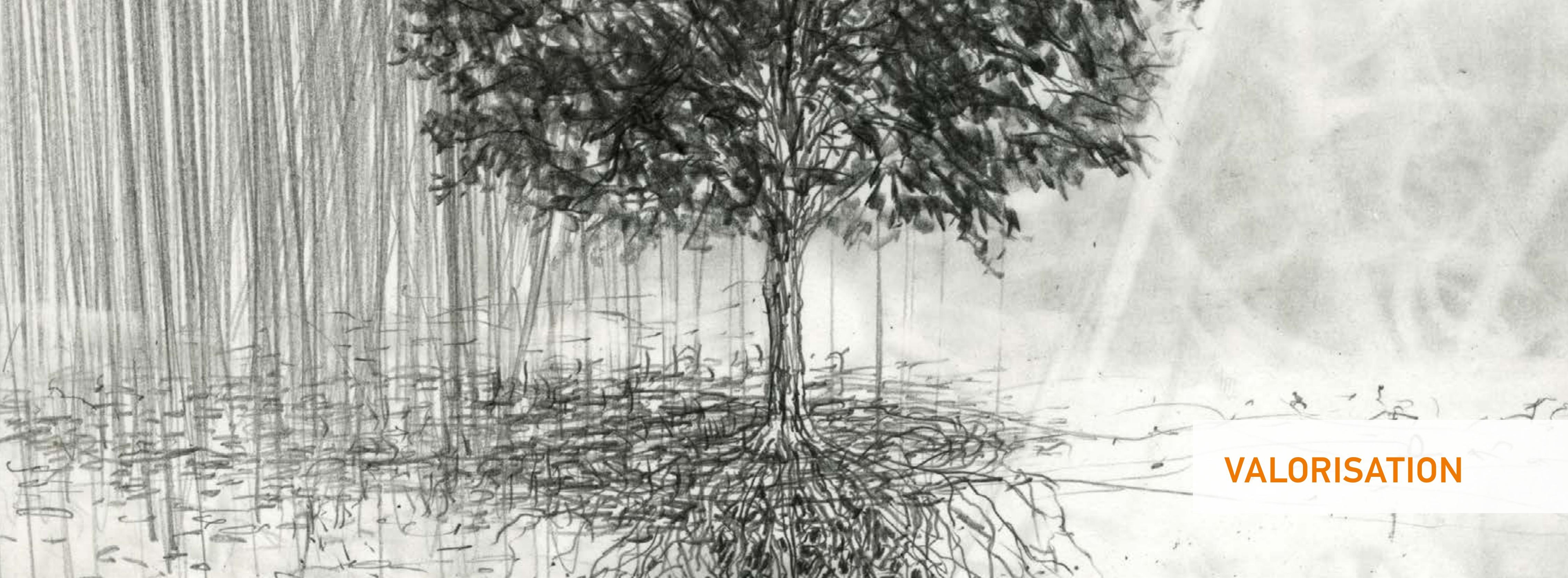




\section{INTRODUCTION}

In 2005 the Dutch Ministry of Science defined knowledge transfer and utilization as the third primary task of universities besides research and education. 'The ministry was the first to use the term 'valorisation' in this context, which refers to the transfer of research knowledge to create societal and/or economic benefits or impact. Comparable terms used in other countries include, amongst others, knowledge exchange, social impact or third mission.?

The official definition of 'valorisation' used by the Dutch government refers to 'the process of creating value from knowledge by making it suitable and/or available for economic and/or societal use and translating it into competitive products, services, processes and entrepreneurial activity.3.

Thereby, valorisation focuses on activities that use novel research knowledge to create additional value on a societal, technical and/or economic level. These different levels are not separated but intertwined. One example is the transfer of novel digitat health appications into clinical practice: The technology has to be mature and stable enough to be successfully implemented in clinical practice and/or the society but a reasonable business model is at the same time required to foster its marketing and implementation by different stakeholders to generate economic impact. The valorisation topic is also becoming increasingly important for universities with regard to research grant applications, which is reflected, e.g, in the knowledge utilization paragraph issued by the Dutch Organization for Scientific Research (NWO). ${ }^{3}$

This valorisation chapter outlines a dissemination roadmap, that describes how the results of this PhD-project already have been used and

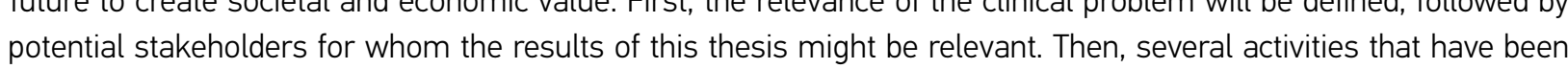
undertaken so far or further need to be undertaken to disseminate the insights and knowledge generated by this project will be described. Finally, innovative aspects and the potential societal and economic value of the research presented in this dissertation will be addressed. As two novel interventions were developed and evaluated in this PhD-thesis, this paragraph will discuss the socio-economic impact of both the clinical framework for (face-to-face) mirror therapy and the telerehabilitation platform ('teletreatment').

RELEVANCE OF THE CLINICAL PROBLEM

The global incidence of all types of lower extremity amputations varies between 6 to 31 per 100.000 in the total population. ${ }^{4}$ Germany ranks in the highest quarter with a total of 56.000 amputations of the lower limb performed in $2011^{5}$ of which around two-thirds were related to
Diabetes. ${ }^{6}$ Reliable data for the incidence of minor and major upper limb amputations in Germany is lacking, but it is estimated that about 6 amputations per 100.000 persons are performed annually in the general population. 'Up to $80 \%$ of all upper and lower limb amputees suffer from phantom limb pain ${ }^{8,9}$ that occurs during the first weeks following amputation and persists over many years in the majority of patients. According to a recent study' in including a mixed sample of upper and lower limb amputees with an average time since amputation of 33 years, $63 \%$ of patients was still suffering from phantom limb pain, which limited their daily routines, functioning, employment and quality of life. ${ }^{11-13}$ The majority of patients receives conventional pharmacological interventions including strong pain medication such as opioids that often results in adverse events, and evidence regarding its long-term efficacy is low..$^{14}$ In this context, non-pharmacological interventions such as mirror therapy that can be used by patients themselves, should also be considered in the treatment of phantom limb pain. Given the chronic nature of this condition, mirror therapy should be delivered on a regular basis over several weeks to months. However, the following clinical problems regarding the delivery of mirror therapy in patients with phantom limb pain can be identified: (1) the evidence for effects of mirror therapy to reduce phantom limb pain is insufficient; (2) little is known about important clinical aspects of the intervention; (3) a standardized evidence-based treatment protocol is lacking; (4) personal resources are often insufficient to provide face-to-face therapy with sufficient dose and (5) adherence to self-delivered exercises is generally poor.

The use of information and communication technology such as telerehabilitation has been proposed to facilitate self-delivered exercises to enhance training intensity. However, little is known about potential benefits of using telerehabilitation in patients with phantom limb pain, and controlled clinical trials investigating effects are lacking.

The findings from this dissertation might help to solve some of the clinical problems described above as they (1) contribute to the evidence (2) present a clinical framework that can be used by heatthcare professionals to personalize mirror therapy in daily care and (3) increase the knowledge about the potential of telerehabilitation to create a personalized blended care intervention for patients with phantom limb pain.

\section{TARGET GROUP AND OTHER STAKEHOLDERS}

The findings of this PhD-thesis might be relevant for several stakeholders as described below.

Patients and their relatives

The two novel interventions that were developed and described in this dissertation put patients with phantom limb pain following amputation in the center of the treatment. They aim to empower patients to actively self-manage their phantom limb pain, as an alternative to standard 
pain medication. Patients often need to perform self-delivered mirror therapy on a regular basis in addition to face-to-face sessions with the personal therapist. The clinical framework for mirror therapy presented in this PhD-thesis serves as the foundation to develop a personalized exercise program for these face-to-face sessions and self-delivered exercises of patients In addition, the teletreatment might be used by eligible patients as an additional tool to support self-delivered exercises and to enhance training intensity. Furthermore, patients who are in general not able to visit the therapist several times in person, might use the teletreatment to enable remote self-management of phantom limb pain. In many cases, relatives and other informal caregivers support patients regarding the self-delivery of mirror therapy at home (e.g. through the application of sensory stimuli) or the use of the teletreatment (e g. technical support). It has been suggested that familymediated exercises are a useful addition to face-to-face sessions. ${ }^{15}$ Therefore, the clinical framework for mirror therapy including a patient log and leaflet to support and monitor self-delivered exercises, and the teletreatment including a manual to facilitate its self-directed use are also relevant for the patient's relatives. Finally the framework for mirror therapy and the teletreatment might also be used by patients with theoretical rationale behind the intervention is similar. ${ }^{16}$

\section{Health care professionals and institutions}

Many different care professionals are involved in the rehabilitation of patients following amputation. In addition to physical and occupational and prosthetists a a phos physiatists, psychologists confronted with the clinical problem of phantom limb pain and strive to offer patients potential solutions using structured (evidence based) treatment protocols. There is a strong demand from professionals working in routine care regarding clinical frameworks or protocols that support health care professionals in the structured delivery of the intervention. This can be seen in the frequent downloads of the open access publications of our clinical framework for mirror therapy in patients with stroke and phantom limb pain, that reached more than 45000 reads by health care professionals around the world five years after their publication. Hence, the knowledge regarding important patient characteristics and the potential of the clinicel framework and the teltreatment in treating phantom limb pain should be disseminated to all professionals involed in the rehailtation of amputees This might increase the knowledge and awareness with respect to ned pharmacolon to nonby different disciplines. The clinical framework and teletreatment incleding a structured manal might also guide (futere) professionals with less experience in how to select eligible patients and how to deliver the intervention. Given the limited resources (time and/or personnel) in clinical practice, health care institutions might consider to use the teletreatment to complement guided therapy and facilitate self-delivery of exercises depending on patient characteristics such as age and computer literacy, preferences and needs.

\section{Students and health care education}

Many universities educating future professionals involved in amputee care (e.g. physical and occupational therapists) have not yet systematically integrated the treatment of phantom limb pain into their curricula. Thus, universities might use the results of this thesis to provide students with knowledge and skills regarding non-pharmacological treatment options to treat phantom limb pain. The clinical framework for mirror therapy and the teletreatment could serve as a guideline how to structure the intervention and to deliver a personalized blended treatment based on patient preferences. Lecturers might use the clinical framework as an example for clinical decision-making according to the different phases in methodical intervention defined by the Royal Dutch Society for Physical Therapy.

Regarding the teletreatment that was developed and evaluated in this dissertation, positive and negative experiences about its use in routine care should be shared with students and teachers from different disciplines. This would provide important insights and knowledge about the thealth interventions in clinical practice, which in turn would facilitate further development implementation and upscaling of digital health.

Finally. lecturers might use the usercentered design process of the teletreatment as an example to illustrate the importance of interprofessional co-creation when designing novel user-friendly digital health interventions. Students and lecturers from different faculties beyond health friendly solutions and appropriate business models.

Researchers

Several future research questions emerged from the different studies performed in this PhD-thesis that might be addressed by upcoming studies. One example is the result from our randomized trial suggesting that three subgroups might benefit more from mirror therapy than others. These subgroups might be validated in future studies to identify responders and to devep a more personalized treatment Furthermore, our clinical framework might serve as guideline for designing an intervention protocol for mirror therapy for future trials 
of its use in clinical practice might inform future digital health studies about important aspects that need to be considered in the design of the study such as sufficient stakeholder involvement, training and support. The studies from this dissertation further illustrate the value of combining different qualitative and quantitative methods during the design and evaluation of the interventions in clinical practice. In addition, the results from this dissertation suggest that alternative research designs to the traditional randomized controlled trial paradigm should be considered when developing future studies investigating the impact and effects of digital health applications. Non-pharmacological
interventions, in particular digital health applications, are developing fast. However, adherence of the end-users to these novel interventions is generally low. Improving adherence and uptake of digital health warrants personalized interventions and close collaboration between different stakeholders from industry, clinical practice and science ${ }^{17}$ as was the case in the PACT project. Finally, the findings from this PhDpain.

Prosthetic manufacturers and other industries

In the past years, traditional manufacturers of prosthetics are facing increased market competition through smaller enterprises and recent technological advancements such as $3 \mathrm{D}$ printers, disrupting traditional business models. Therefore, these corporates are seeking new business models or try to expand their existing business model by eg. offering clients additional products or services that create unique selling points or long-term relationships. This might create new collaborations between traditional (bigger) corporates and smaller many cases more agile enterprises (eg digital health developers). Beyond this, these companies have in many cases their own physical or occupational therapists who also treat patients from other countries, in which the (paralmedical infrastructure is less elaborated However these multinational companies need to ensure high-quality care of amputees in all markets in which they are active. The teletreatment presented in this dissert (a) lients and to deliver remote traning and care. To this end the teletreatment might be complemented with additionat more prosthetic-specifc content such as prosthetic training and care.

Thesights generated from the process evaluation regarding the teletreatment presented in this dissertation might help the soffware co be improved by nosd maintenance as operating systems and hardware are also rapidly evolving. These software updets 列 rs and their relatives need sufficient training and support regarding the use of the technology, to enable successful implementation in routine care.

Health insurance companies

The majority of amputees suffers from phantom limb pain for many years and the average annual costs per patient associated with the standard pharmacological treatment are estimated at around 1.000 Euro (unpublished data German health insurance company). Additional costs associated with phantom limb pain that are covered by health insurances are caused by medical products such as residual limb liners made from electromagnetic shielding fabric, (para)medical treatment or disability payment in case of absence from work. The two novel interventions presented in this PhD-thesis that aim to support self-management of patients with phantom limb pain might therefore also approaches play an increasingly important role in the management of chronic pain.18

\section{DISSEMINATION OF FINDINGS}

The consortium of the PACT project consisted of many different partners from patient associations, clinical practice, education, research, and the industry. As such, constant knowledge exchange occurred on many different levels between the different partners through various activities such as the user-centered design process of the clinical framework and the teletreatment, project meetings or student projects. In the following paragraph the activities that already have been performed as well as future activities to disseminate the findings from this PhD-thesis are described. In addition to these activities, several products and services are presented into which the research findings have been or will be translated.

Activities performed so far

The findings from the different phases of the PACT project have already been distributed through various channels to different stakeholders such as patients, health care professionals, students and researchers as shown in table 1. 
Table 1. Overview of dissemination activities that have been performed in the PACT project

\begin{tabular}{|c|c|}
\hline & Knowledge transfer to patients and society \\
\hline Activities & Description \\
\hline Online blog & $\begin{array}{l}\text { During the PACT project news and interesting facts have continuously been posted in a blog } \\
\text { (http://telerehanent/) including a newsletter }\end{array}$ \\
\hline TV and radio appearances & $\begin{array}{l}\text { The project and its (preliminary) results have been presented in three German } \\
\text { National TV reports (WDR Servicezeit 'Hightech in der Medizin': https://www.youtube. } \\
\text { com/watch?v=cY7ui-nbuHw\&t=41s, Medical Travel RTL 4: https://multimavision. } \\
\text { nl/medicaltravel/, ZDF Infokanal: der elektrische Reporter: https://wwww.youtube. } \\
\text { com/playlist?list=PL4195823E4A32DBC2) and one German radio post: https://www. } \\
\text { deutschlandfunkkuttur.de/sendungsuberblick-virtueller-korpertausch-do-it-yourself.1264. } \\
\text { de.html?dram:article_id=405201 }\end{array}$ \\
\hline $\begin{array}{l}\text { Interview, video recording and } \\
\text { presentations at medical fairs }\end{array}$ & $\begin{array}{l}\text { The project has been presented several times to the public at a booth of the world's biggest } \\
\text { medical fair MEDICA in Düsseldorf and the 'IT Trends' in Essen. In addition, an interview and } \\
\text { video recording including the patient representative of the PACT project took place: https:// } \\
\text { www.youtube.com/watch?v=9rA8smDfkG8\&t=14s }\end{array}$ \\
\hline Newspaper report & $\begin{array}{l}\text { A plain language report about the PACT project was published in the German newspaper } \\
\text { 'Hamburger Abendblatt': https://www.abendblatt.de/ratgeber/wissen/article206579861/ } \\
\text { Zukunftstrends-Die-digitalen-Arzthelfer-kommen.html }\end{array}$ \\
\hline Online articles in plain language & $\begin{array}{l}\text { Several online articles e.g. on the website of the biggest patient association for amputees } \\
\text { in Germany have been published: https://www.bmab.de/news } 118 / \text {, http://rehanews } 24 \text {. } \\
\text { de/das-bein-ist-weg-der-schmerz-bleibt//, htps:// egesundheit.nrw.de/projekt/telereha- } \\
\text { phantomschmerz/ }\end{array}$ \\
\hline Public debates & $\begin{array}{l}\text { Several panel discussions about the PACT project took place (e.g. Dutch eHealth week 2016, } \\
\text { Dutch-German Innovation Days on Digitalization in Healthcare, Creative health conference) }\end{array}$ \\
\hline
\end{tabular}

Activities Description

Dissemination through patient

representative

A patient representative was actively involved during all phases of the PACT project and various dissemination activities. For example, a public stand-up paddling event for com/watch?v=zmBDhHdbH1M

\section{Knowledge transfer to health care professionals and clinical practice}

Publications in national

professional journals

Three articles regarding the practical use of the framework and teletreatment have been published in the German national journal for physical therapists, prosthetists and general practitioners.

Presentation, workshop and

debates at national conferences

The PACT project has also been presented at the Annual conference of the German Association for Hand therapy in Düsseldorf 2013, where also a workshop about mirror therapy took place for physical and occupational therapists. In addition, an online presentation was given at the 15th National physical therapy congress in Cambodia.

Implementation in routine care through practical workshops for health care professionals and online register Several practical workshops about the clinical framework for mirror therapy and the teletreatment were organized for education and training centers and rehabilitation clinics in Germany and the Netherlands. At the moment, several German health care institutions and prosthetists use the clinical framework for mirror therapy and the teletreatment in routine care. These practitioners are listed in an online mirror therapy register. http.// spiegeltherapie.com/therapeutenverzeichnis/

Open access publication clinical

framework for mirror therapy in

The have been published open access on ResearchGate. They have reached more than 45000 reads by health care professionals, researchers and educators around the world. 


\section{Knowledge transfer to education}

Practical workshops

Web lecture

Inclusion of students in

graduation projects

\section{Publication in peer-reviewed}

journals

Presentations at (inter)national

scientific conferences

\section{Collaboration with software}

company

Release of iOS and Android App
Several practical workshops about the clinical framework for mirror therapy and the

teletreatment were organized for physical therapy students of Zuyd University Heerle (regular Bachelor and German EPEPE program) and rehabilitation management students of A whive

A web lecture about the theoretical foundation of mirror therapy (e.g. evidence and A europhysilogical mechanisms) in patients with phantom limb pain was developed for

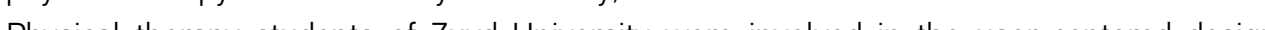
Physical therapy students of Zuyd Universty were involved in the user-centered design .

\section{Knowledge transfer to research community}

All six articles included in this thesis have been published in international, peer-reviewed journals,

The PACT project and its results have also been presented and discussed at (inter)national conferences focusing on pain research (e.g. 7th World Congress of World Institute of Pain 2014, German Pain Congress Hamburg 2014, Myosens Symposium Göttingen 2015.

\section{Knowledge transfer to industry and health insurances}

The development and evaluation of the teletreatment occurred in close collaboration with a software company (Kaasa health, Germany).

At the end of the PACT project a revised version of the teletreatment was released in the App Store ${ }^{\oplus:}$ https://itunes.apple.com/de/app/routine/id 1 152443756?mt=8

In the meanwhile, a modified version of the App has also been released for patients with

\begin{tabular}{ll}
\hline Activities & Description \\
\hline $\begin{array}{l}\text { chronic pain of the upper limb: https://itunes.apple.com/de/app/routine-health/ } \\
\text { id1446256495\#?platform=ipad }\end{array}$ & $\begin{array}{l}\text { The publications from the PACT project have been disseminated to several health } \\
\text { insurances and industry partners involved in the project. } \\
\text { industry and health insurance } \\
\text { companies }\end{array}$ \\
$\begin{array}{l}\text { Negotiations with health } \\
\text { insurance companies and } \\
\text { prosthetic manufacturers }\end{array}$ & $\begin{array}{l}\text { During the PACT trial, negotiations with several German health insurance companies } \\
\text { and prosthetic manufacturers about potential reimbursement and business models for } \\
\text { the teletreatment took place. Two months after funding of the project ended, the first } \\
\text { reimbursement was achieved. At the moment, the blended care program combining } \\
\text { face-to-face mirror therapy and the teletreatment is reimbursed by several health } \\
\text { insurances in Germany and is used by a variety of health care institutions in routine care. }\end{array}$
\end{tabular}

Future dissemination and implementation activities

The following paragraph describes several future dissemination activities to further upscale the implementation and use of knowledge that was gathered in the PACT project.

\section{Knowledge transfer to patients and society}

The results of this PhD-thesis will further be disseminated to the public by press releases of Maastricht University and Zuyd University. Furthermore, the most important results will also be posted on the PACT blog (http://telereha.net/) and the mirror therapy website (http:// spiegeltherapie com) The PhD-thesis will be accessible worldwide via the research portal repository of Maastricht University (htps.//cris. maastrichtuniversity.n//portal//). In addition, the thesis including a plain language summary will be disseminated to various partners from the PACT consortium, including the funding institution and the biggest patient association in Germany. 
Knowledge transfer to health care professionals and clinical practice

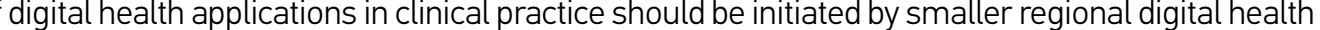

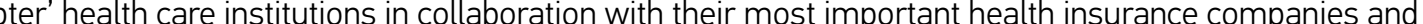
other cooperating institutions. Therefore, additional health care professionals in Germany and the Netherlands will be trained regarding the delivery of the clinical framework and the teletreatment to foster their use and implementation in clinical practice. Communities of practice amongst health care professionals using the teletreatment will be set up to exchange experiences concerning its use in routine care. In addition, an online register of practitioners using the teletreatment will be created, so that different stakeholders are informed about whom they can contact for more information about the intervention and set up potential collaborations.

The software company that currently commercializes the teletreatment, strives to achieve additional contracts with health insurance companies, so that a wider group of patients and health care professionals in Germany the Netherlands and beyond are able to make use of of the Royal Dutch Society for Physical Therapy. Thereby, a wide range of physical therapists in the Netherlands will be informed about the centers (e.g. Adelante Centre of Expertise in Pain and Rehabilitation).

\section{Knowledge transfer to education}

A web lecture and workshop about the clinical framework for mirror therapy and the teletreatment have already been developed and performed experiences gathered through the user-centered design process of the teletreatment will be used as an example within a master class about Zuyd University. In the future, other stakeholders and clients might also enroll in this masterclass.

\section{Knowledge transfer to the research community}

Upcoming press releases of Maastricht University and Zuyd University will also inform the research community about the results of this dissertion. In 2020, a presentation about the PACT project will take place at the Orthep

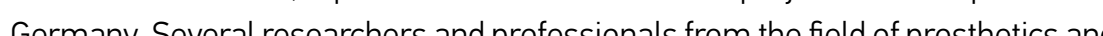

providing an overview of the PACT project and its results will be published open access to inform researchers worldwide about the knowledge gathered in this PhD-thesis.

Multi-stakeholder business models incorporating health insurances, industry, health care institutions and the end-users should be considered in further implementation of the teletreatment in routine care. The ultimate goal is to create smaller regional digital health ecosystems with the relevant stakeholders from clinical practice, industry and research. Therefore, the results from this dissertation will be disseminated to additional German and Dutch health insurance companies in order to discuss potential reimbursement models regarding
the teletreatment. Prosthetic manufacturers and orthopaedic technicians collaborating with health care institutions treating amputees will also be informed bout the results of this PhD-thesis. The experiences from the delivery of the interventions in routine care should be shared with all stakeholders involved to further improve and upscale the clinical framework and the teletreatment

\section{INNOVATIVE ASPECTS}

The following paragraph discusses several innovative aspects of the results presented in this thesis in relation to existing activities, services and products.

The clinical framework for mirror therapy in patients with phantom limb pain was developed based on the best available evidence, clinical developed using rapists and patient preferences. It is to our knowledge the first framework in the treatment of chr supports clinical decision making and can directly be integrated into the daily work of physical and occupational therapists which is embraced by many professionals. What clinical frameworks distinguishes from more rigid protocols is their flexibility to tailor the intervention to the characteristics and needs of individual patients seen in routine care. However, not many clinical frameworks have been developed and (1) in neurological rehabilitation. ${ }^{19,20}$

Successful development of digital health applications needs the composition of unconventional teams, trans-institutional initiatives and 
crossing conventional barriers between disciplines and funding sources. ${ }^{17}$ However, in many digital health projects co-creation together with the end-users and other stakeholders is not self-evident. The novel teletreatment presented in this dissertation was developed in close cocreation with different stakeholders including a patient representative, who were involved in all phases of the project. This close collaboration with different stakeholders ensured commitment to the project and continuous feedback on the design of the teletreatment. A novel 'product' that was developed and applied during the design process of the teletreatment is an innovative multi-stakeholder decision matrix that enables structured prioritization of user requirements. The novel aspect of this matrix is in our opinion, that the perspectives of different stakeholders within such a digital health project are taken into account. One example is the technical complexity of each requirement in terms of time and/ or money needed which is rated by the software developer. Furthermore, the lessons learned from the teletreatment development phase of the PACT project point out some important and novel aspects (eg. early process evaluations and sufficient experience of professionals) that should be considered by future digital health projects to improve novel technology-driven interventions and the outcomes of studies investigating their impact.

In the digital health sector, there is currently a clear trend towards mobile health applications. Tablets and smartphones are more and more becoming the preferred devices for interactions between health care professionals and the end-users. The teletreatment presented in this dissertation is to our knowledge at present the only mobile health application for patients with phantom limb pain that is reimbursed by health insurance companies and already partly implemented in routine care. 


\section{REFERENCES}

Ministry of Education Culture and Science. Valorisatie van onderzoek als taak van de universiteiten. The Hague 2005.

De Jong S. Engaging scientists: Organising valorisation in the Netherlands. The Hague: Rathenau Institute: Doctoral thesis, Leiden

Nederland Ondernemend Innovatieland. Van voornemens naar voorsprong: kennis moet circuleren. Den Haag: Inderdepartmentale Programmadirectie Kennis en Innovatie 2009.

Moxey PW Goganiceanu P. Hinchlife RJ et al Lower extremity amputations--a review of global variability in incidence. Diabet Med 2011; 28: 1144-53.

Spoden M, Nimptsch U and Mansky T. Amputation rates of the lower limb by amputation level - observational study using German national hospital discharge data from 2005 to 2015 . BMC Health Serv Res. 2019; 19:8.

Claessen H, Narres M, Haastert B, et al. Lower-extremity amputations in people with and without diabetes in Germany, 2008-2012 - an analysis of more than 30 million inhabitants. Clin Epidemiol. 2018; 10: 475-88.

Schnetzke M, Hernekamp J, Grützner P and Gühring T. Majoramputationen der oberen Extremität. Orthopädie und Unfallchirurgie up2date. 2016; 11:3-24.

Ephraim PL, Wegener ST, Mackenzie EJ, Dillingham TR and Pezzin LE. Phantom pain, residual limb pain, and back pain in amputees: results of a national survey. Arch Phys Med Rehabil. 2005; 86: 1910-9.

Richardson C, Glenn S, Nurmikko T and Horgan M. Incidence of phantom phenomena incluaing phantom limb pain 6 months after major lower limb amputation in patients with peripheral vascular disease. Clin J Pain. 2006; 22: 353-8,

(0) recall of animpared body representation in dreams-results from a nation-wide survey on limb amputees. PloS one. 2015; 10: e0119552.

Christensen J, I psen T, Doherty P and Langberg H. Physical and social factors determining quality of life for veterans with lower-limb amputation(s): a systematic review. Disabil Rehabil. 2016; 38: 2345-53.

2. Rothgangel A, Braun S, Winkens B, Beurskens A and Smeets R. Traditional and augmented reality mirror therapy for patients with chronic phantom limb pain (PACT study): results of a three-group, multicentre single-blind randomized controlled trial. Clin Rehabil. 2018; 32: 1591-608.
13. Whyte AS and Carroll LJ. A preliminary examination of the relationship between employment, pain and disability in an amputee population. Disabil Rehabil. 2002; 24: 462-70.

Alviar MJ, Hale T and Dungca M. Pharmacologic interventions for treating phantom limb pain. Cochrane Database Syst Rev. 2016 10: $\mathrm{CD} 006380$

15. Galvin R, Cusack T, O'Grady E, Murphy TB and Stokes E. Family-mediated exercise intervention (FAME): evaluation of a novel form of exercise delivery after stroke. Stroke. 2011; 42: 681-6.

16. Thieme H, Morkisch N, Rietz C, Dohle C and Borgetto B. The Eficacy of Movement Representation Techniques for Treatment of Limb Pain--A Systematic Review and Meta-Analysis. J Pain. 2016; 17: 167-80.

17. Nationale Wetenschapsagenda (NWA). Gezondheidszorgonderzoek, preventie en behandeling. Den Haag 2018.

18. Nicholas MK and Blyth FM. Are self-management strategies effective in chronic pain treatment? Pain Manag. 2016; 6: 75-88.

19. Braun S, Kleynen M, Schols J, Schack T, Beurskens A and Wade D. Using mental practice in stroke rehabilitation: a framework. Cl Rehabil. 2008; 22. 579-91.

20. Kleynen M, Beurskens A, Olijve H, Kamphuis J and Braun S. Application of motor learning in neurorehabilitation: a framework for health-care professionals. Physiother Theory Pract. 2018: 1-20. 


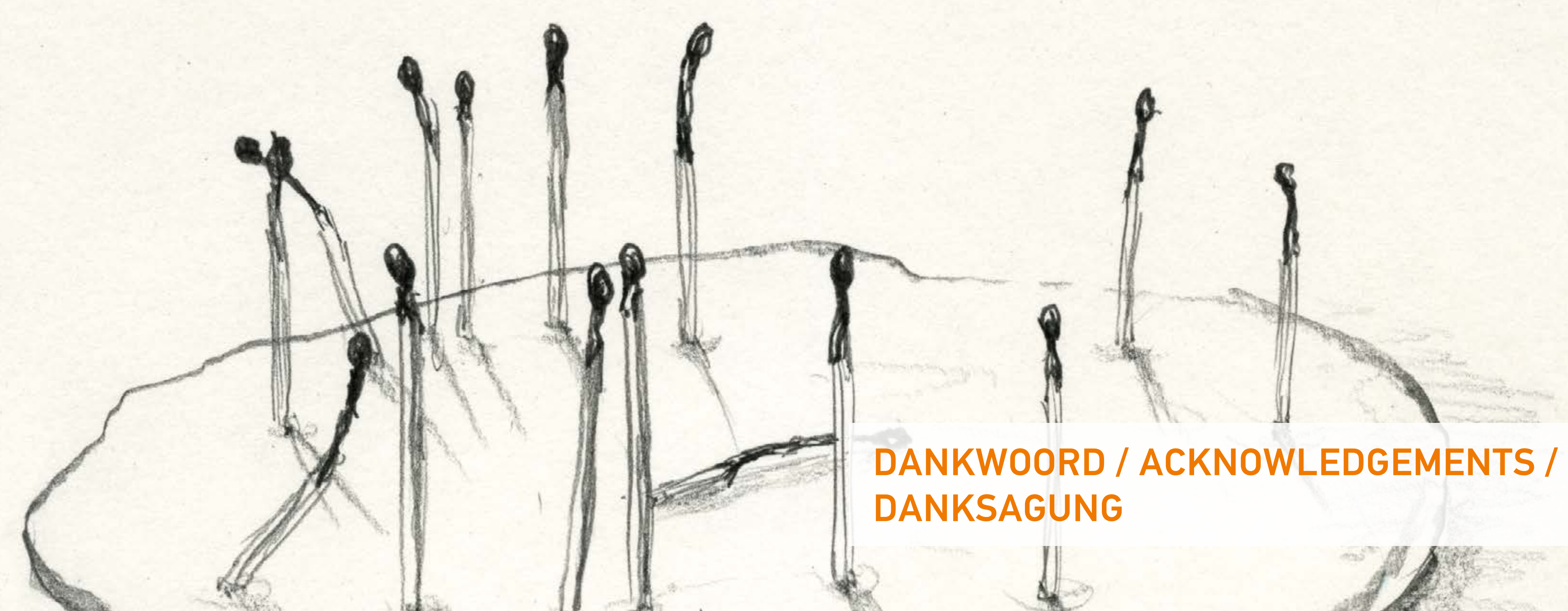


Nu ik zover ben gekomen om het dankwoord voor mijn proefschrift te schrijven, kijk ik terug op 9 leerzame en spannende jaren met veel emotionele up en downs. Het PACT project begon met de toekenning van een grote subsidie, tegelijkertijd werd ik voor de eerste keer vader. De laatste twee jaren van het project waren een grote uitdaging in verband met de ziekte van mijn geliefde vrouw Sarah en de geboore van van de gelegenheid gebruik maken om iedereen die heeft bijgedragen aan dit proefschrift van harte te bedanken.

Now that I have come so far to write the acknowledgement paragraph for my dissertation, I look back on 9 instructive and exciting years with many emotional up and downs. The PACT project started with the award of a large grant, at the same time I became a father for the first time. However, the last two years of the project were a big challenge because of the illness of my beloved wife Sarah and the birth of our third son Emil Without the support of my PhD-team, colleagues, family and friends, I would never have come that far and I would like to take this opportunity to thank everyone who contributed to this dissertation.

Ik ben erg dankbaar voor mijn geweldige promotieteam bestaande uit Sandra Beurskens, Rob Smeets en Susy Braun. Door jullie ondersteuning ben ik over de jaren inhoudelijk maar ook persoonlijk sterk gegroeid.Jullie hebben mij altijd de ruimte gegeven om mijn eigen ideë̈n en visies te verwezenlikken Ook Luc de Witte wil ik hierhij niet vergeten, die de eerste jaren nauw betrokken was bij dit PhD-project.

Sandra, jij was al bij mijn afstudeerproject voor de opleiding fysiotherapie betrokken en wij hebben samen de scriptieprijs van het KNGF in 2002 gewonnen. Nu 17 jaar later mogen we weer samen feesten. Aan het begin van het PhD-project toen er nog geen subsidie was, heb jij je samen met Susy sterk gemakt vor het regen van een promotievoucher bij Zuyd Dit heeft mil de kans gegeven om überhaupte goede start met de promotie te kunnen mak Door jouw grote expertise op het gabied van doelgericht meten patiënt- en praktijkgerich

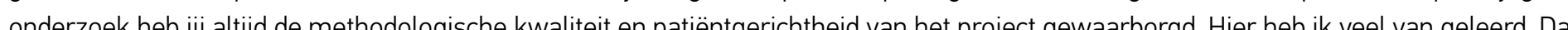

Rob, ik ben dankbaar voor je humor en je motiverende aard, die mij erg geholpen hebben om vol te houden en soms de dingen iets pragmatischer te zien. Door jouw ontzettend groot netwerk in de piin revalidatie wist iij altijid een goede contactpersoon voor in wetenschappelijke tijdschriften, clinici die wij konden betrekken of relevante pijn congressen. Zo heb jij onder andere ervoor gezorgd dat
Ik op het congres van het World Institute of Pain mocht spreken. Na toekenning van de PACT subsidie ben jij zelfs met Susy naar Düsseldorf gekomen om bij de plechtige uitreiking van de oorkonde aanwezig te zijn. Jij had altijd nog aanvullende ideeën hoe wij met ons onderzoek nog meer impact konden genereren. Dank voor jouw inzet en passie voor het onderwerp en ik hoop dat wij in de toekomst nogmaals de kans krijgen om samen te werken.

Dan Susy, wij kennen elkaar inmiddels 20 jaar (!). Jij was al mijn docent tijdens de opleiding fysiotherapie en ik heb je sindsdien altijd ervaren als iemand met een uitzonderlijke passie voor de inhoud, een goed sociaal team en samenwerking met andere disciplines. Jij was vanaf de eerste minuut bij het PACT project betrokken, zelfs op een voor jouw privé zwaarste dagen ben iij samen met mij naar de subsidiegever in Jülich gereden en daarna hebben wij van een van je andere passies genoten: Laugenstangen! Als mijn dagelijkse begeleider heb jij altijd voor een duidelijke structuur in de uitvoering van het project (en mijn geest) gezorgd. Jij was altijd aanspreekbaar, zelfs avonds of in de

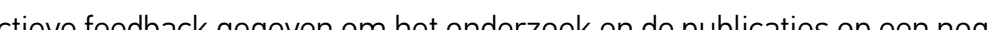
hoger nivea te tillen, wardoor ik veel van jouw geleerd heb en je hiervoor erg dankbaar ben Ik bewonder hoe jij altijid ervoor zorgt dat mensen uitjouw team de dingen kunnen (blijven) doen die zij leuk vinden en waar hun hart sneller van gaat kloppen. Door kleine en grote attenties, warme, motiverende gesprekken en vel empathie (en koffe) drag je zorg dat het werk

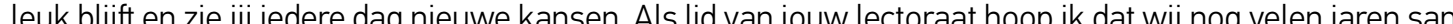
doen en daarbij onze humor en andere leuke dingen behouden.

Luc, jij was ook intensief betrokken in de planning en uitvoering van het PACT project, voordat jij naar Sheffield ging. Met jouw ontzettend oprechte dank.

Dear Derick, I am very thankful that you were part of the 'early' PhD-team involved in first thoughts and ideas about my dissertation and that I had the chance to publish my first article together with you as co-author. I learned a lot from this early phase in my PhD career.

Furthermore, I would like to thank the assessment commitee, Prof. Clemens Rommers, Prof. Johan Vlaeyen, Prof. Klasien Horstman, Prof. 
Catherine Bolman, Dr. Lennard Voogt and Dr. Susann Seddigh for being part of the corona of my PhD defence.

Graag bedank ik ook alle collega's van het cluster lectoraat en de vakgroep revalidatiegeneeskunde van de Universiteit Maastricht voor jullie collegialiteit en meedenken in verschillende fasen van het promotieonderzoek. In het bijzonder wil ik de collega's van het lectoraat voeding. leefstijl en bewegen bedanken, jullie hebben mij steeds met tips, eigen voorbeelden en door het overnemen van taken geholpen dat ik weer een slag kon maken met het afmaken van mijn proefschrift.

Lieve collega's en mede-promovendi (of gepromoveerden) Melanie, Renée, Iris, Tom, Li-Juan, Steffy, Stephie, Jerôme, Anita, Emmylou, Stephanie, Barbara, Jolanda, Linda en Barbara dank voor jullie ideeen, meedenken en delen van voorbeelden, kennis en ervaringen uit julte eigen promotietraject. Graag wil ik ook Sanne van der Weegen, Laura Hochstenbach en Renee Verwey bedanken voor hun inspirerende ondersteuning binnen het gebruiker-gecentreerde designproces van de technologie.

Graag bedank ik Marieke Spreeuwenberg voor de ondersteuning in de sample size calculatie en Marielle Goosens voor de ondersteuning in de kosten-batenanalyse tijdens de planning van ons clinical trial. Björn Winkens voor de complexe statistische analyses en leuke grapjes tussendoor.

En natuurlijk een grote dank aan de goede feeën van het cluster lectoraat en de vakgroep revalidatiegeneeskunde van de Universiteit . Ber

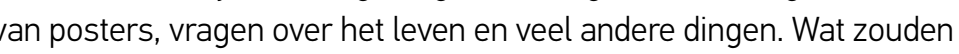
we toch zonder jullie moeten.

Mijn oprechte dank ook aan de lieve collega's van de opleiding fysiotherapie voor jullie interesse in het onderzoeksproject, de emotionele ondersteuning en gezelligheid gedurende de laatste jaren. Mijn kamergenoten, Miekie, Josine, Emmylou, Esther, Simone, Juliëtte, Ruud en John wil ik danken voor alle gesprekken, mentale ondersteuning en de leuke etentjes buitenshuis. Het was erg gezellig!

Gaston en Tom van Maastricht Academy of Media Design and Technology, dank voor de inspirerende talks (in het busje en andere locaties) en het delen van jullie ideeën rondom co-creatie en design thinking.
Diese Dissertation wäre ohne eine finanzielle Förderung nicht möglich gewesen. Deswegen möchte ich mich ganz herzlich bedanken bei Cornelia Schlebusch, Jutta von Reis, Eva Michalak, Stefanie Kurth, Isabell Backes und Harald Zillikens vom Projektträger ETN der Leitmarktagentur Nordrhein-Westfalen und dem Ministerium für Gesundheit, Emanzipation, Pflege und Alter des Landes NordrheinWestfalen in Düsseldorf, insbesondere bei der ehemaligen Gesundheitsministerin Barbara Steffens. Sie haben für eine reibungslose und angenehme Projektabwicklung des PACT Projekts gesorgt und standen mir immer mit Rat und Tat zur Seite. Durch ihre Förderung und ihr Netzwerk konnten wir bereits während der Projektlaufzeit eine hohe öffentlichkeitswirksamkeit erzielen, wie zum Beispiel mit unserem Stand auf der MEDICA. Ich würde mich freuen, wenn wir auch in Zukunft gemeinsame Projekte vorantreiben könnten.

Tevens ben ik de Faculteit Gezondheidszorg van Zuyd Hogeschool erg dankbaar, in het bijzonder Peter Hilderink, Raymond Clement en de teamleiders Monique van den Broek en Claudy Cobben die mij o. a door het toekennen van een promotievoucher ruimte en mogelijkeden een workshop in het curriculum van de reguliere opleiding tysiotherapie en het EPEPE-programma te integreren die de nieu be kennis en inzichten uit dit PhD-project terug laat vloeien naar het onderwijs.

Zusätzliche finanzielle Starthilfe zu Beginn dieser Dissertation habe ich der ZVK-Stiftung, insbesondere Prof. Dr. Erwin Scherfer, zu verdanken. Hiermit konnte ein erster wichtiger Grundstein für weitere Förderanträge gelegt werden.

Im Rahmen des PACT Projekts wurde eine der weltweiten größten Therapiestudien zu nicht-medikamentösen Behandlungen bei denen ich mich ganz herzlich bedanken möchte:

Prof. Greitemann, Michaela Joswig, Frank Assmann, Helga Kaiser und das gesamte Physio-Team der Klinik Münsterland Bad Rothenfelde herzlichen Dank für die professionelle, strukturierte und immer persönliche Zusammenarbeit und die humorvollen persönlichen Gespräche und die vielen Telefonate, die wir geführt haben

Prof. Schulz, Alexandra Kriha, Andrea Pinner, Khira Jung und das gesamte Team der Ergotherapie des St. Marien-Hospitals Köln, herzlichen 
Dank auch für die Unterstützung des Ethikantrags und die unkomplizierte und reibungslose Organisation der PACT Projekttreffen in Ihrer Klinik!

Dr. Simmel, Gabriele Zankl, Armin ter Haseborg, Hans Baumgärtler, Ulrich Ernst, Meike Schellenberger, Isabel Burchardt und das gesamte Ergotherapie Team der Berufsgenossenschaftlichen Unfallklinik Murnau. Vielen Dank für die engagierte Mitarbeit über das gesamte Projekt sowie die Informationsveranstaltungen zum PACT Projekt in Ihrem Hause. Herzlichen Dank auch für Unterstützung hinsichtlich einer möglichen Kostenübernahme der Telereha bei der Deutschen Gesetzlichen Unfallversicherung und die Gespräche im Biergarten Ähndl mit Blick auf die Berge!

Prof. Bouillon, Dr. Fabian, Andrea Meinold und Horst Cupan (Kliniken der Stadt Köln), vielen Dank für die tatkrättige Unterstützung der Betreuung der Patientinnen und Patienten und die freundschaftliche Ebene, auf der wir miteinander kommuniziert haben.

Ein großes Dankeschön an das gesamte Team von Protheofit in Troisdorf, insbesondere Sandra und Julia Förster, Patrick Punzet und Inga

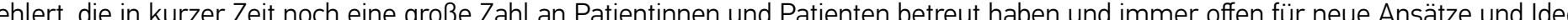
waren.

Dann die Dr. Becker Klinik in Nümbrecht, insbesondere Thomas Claus, Anne Specht, Jana Hofmann und Isabelle Pechlof, die mir bei so vielen Fragen zur Seite standen und immer eine unkomplizierte Lösung parat hatten.

Dr. Johannes Schröter, Bettina Glorius, Julia Jakubasch, Sandra Majkic, Isabel Scheibe und Richard Schlegel (MEDIAN Klaus Miehlke Klinik Wiesbaden), wir kennen uns schon seit meiner Zeit an der Hochschule Fresenius in Idstein. Damals konnten wir bereits erste gemeinsame

Dr. Fischer, Dr. Kuhn, Kay Herold und Kathrin Hanl (BG Klinikum Bergmannstrost Halle), auch wenn die Entfernung zwischen Halle und Düsseldorf sehr weit war, gab es immer kurze Wege in der Kommunikation und die Umsetzung lief reibungslos, vielen Dank!
Stefan Schmitt und Kai Haarhaus von der Arbeitsgemeinschaft Ergotherapie in Mönchengladbach, wir kennen uns auch schon ewig und wir hatten immer ein sehr freundschaftliches Verhältnis. Ich bin froh, dass ihr am Projekt teilgenommen habt und auch einige ambulante Patientinnen und Patienten durch euch kompetent betreut werden konnten.

Bei Susanne Glaudo und Herta Spelter mit ihrer großen klinischen Expertise möchte ich mich ganz herzlich für ihre Beteiligung bei der Erstellung des Behandlungsleiffadens und die vielen inspirierenden Gespräche über die Spiegeltherapie bedanken.

Ohne das Interesse und die Teilnahme von so vielen Patientinnen und Patienten wäre das Projekt natürlich auch nicht möglich gewesen. Daher geht ein ganz großer Dank auch an alle Patientinnen und Patienten, die sich an der Anforderungsanalyse, Entwicklung des Behandlungsleitfadens und der Telereha sowie der abschließenden klinischen Studie beteiligt haben. Besonders möchte ich hierbei unseren Patientenvertreter Dr. Thomas Frey hervorheben, der von der ersten Minute an das Projekt begleitet hat und eine extrem wichtige Rolle innerhallb der Verbreitung der Projektergebnisse spielte. Thomas, herzlichen Dank für die vielen gemeinsamen Veranstaltungen und dein Engagement während des gesamten Projekts!

Daneben geht ein besonderer Dank auch an Detlef Sonnenberg vom Bundesverband für Menschen mit Arm- und Beinamputationen der tatkrättig die Patientenrekrutierung und Verbreitung der Projektergebnisse unterstützt hat.

Dankbar bin ich auch dem Softwareentwickler Kaasa health und Dr. Petra Becker, insbesondere dem, Telereha-Team' Vanessa, Büsra, Caro und den Entwicklern Bastian, Tino, Ingo, Joel, Tobias, Marius, Paul, Jochen und der ,guten Seele' Waltraud. Wir waren ein klasse Team, das in kurzer Zeit ein tolles Produkt entwickelt hat und ich habe euch viel zu verdanken! Es war eine sehr erfolgreiche, intensive Zeit, in der ich sehr viel über die Arbeitsweise in einem agilen Start-up gelernt habe.

Caro, tausend Dank für das tolle Layout dieser Dissertation, du warst immer erreichbar und hast bis zur letzten Minute meine vielen Anmerkungen noch umgesetzt. Das Ergebnis kann sich wirklich sehen lassen!

Auch ein herzliches Dankeschön an alle Studentinnen und Studenten des Studiengangs Physiotherapie der Zuyd University Heerlen, die sich 
am PACT Projekt beteiligt haben, insbesondere Vanessa Waggeling und Rebecca Kahler, die in ihrer Bachelorarbeit die Benutzerfreundlichkeit der Telerehabilitation untersucht haben.

Bedanken möchte ich mich auch bei Mandy Kettlitz (Techniker Krankenkasse), Barbara Hassenkamp (Deutsche Rentenversicherung Westfalen), Elmar Lausen (Barmer GEK), Alexander Meyer (Deutsche Gesetzliche Unfallversicherung), Thomas Zimmermann und Dr. Susanne Kloppel-Wirth (Barmenia) sowie Barbara Nickesen (AOK Rheinland/Hamburg) für ihre Offenheit und Kooperationsbereitschaf mögliche Vergütungswege für die Telerehabilitation zu prüfen.

Der pt_Zeitschrift für Physiotherapeuten, Dr. Tanja Bossmann, Jörg Stanko, Agnes Hey und Frank Aschoff danke ich für die tollen Fachbeiträge in der pt_Zeitschrift und interessanten Interviews und Filmbeiträge über das PACT Projekt, unter anderem auf der MEDICA in Düsseldorf.

Zu guter Letzt möchte ich mich bei einigen Personen aus meinem privaten Umfeld bedanken, die mit ihrer mentalen und physischen Unterstützung maßgeblich dazu beigetragen haben, dass ich diese Doktorarbeit überhaupt fertigstellen konnte.

Meinen engen Freunden und ihren Familien: Alex, Steffen, Christian, Stephan, Jan, Guido, Niels, Frank, Mika, Lukas und alle die ich evtl. noch vergessen habe: Tausend Dank fur die vielen Aufmunterungen, geselligen Abende, motiverenden Gespräche und Witze, die mich immer wieder daran erinnert haben bei der ganzen Arbeit den Spaß nicht zu verlieren Alex vielen Dank für die vielen Jahre enger Freundschaft' Wir haben 2001 das Projekt Spiegetherapie' gemeinsam gestartet, 18 Jahre später bist du. Paranimf' be i der mündlichen Verteiligung meiner Dissertation

Meinen tollen Schwiegereltern Ursula und Jürgen ein unendlicher Dank für die Betreuung von Oskar, Paul und Emil, die Unterstützung im und um das Haus herum und was ihr sonst noch alles für uns gemacht habt. Eure Unterstützung war unverzichtbar und eine große Hilfe für uns!

Mama, Papa und Gregor, tausend Dank für die vielen Gespräche, die wir über die Doktorarbeit geführt haben und eure Unterstützung über die ganzen Jahre hinweg! Ich bin stolz so tolle Eltern und einen so guten Bruder zu haben, ich bin sehr dankbar dafür.
Sarah, ohne Deine Liebe, Geduld, Unterstützung und motivierenden Worte würde ich jetzt nicht diese Zeilen schreiben. Du weißst, wie unglaublich dankbar ich dir dafür bin. Gemeinsam haben wir diese Zeit der unglaublichen Höhen und tiefsten Täler bewältigt. Jetzt ist es endlich vollbracht und Du bist wieder gesund! Was für ein Glück!

Mein lieber Oskar, mit Deiner Geburt 2011 begann für mich ein neuer Lebensabschnitt, die intensivste Zeit meines Lebens. Du hast mich zum Vater gemacht, was für eine Ehre und Herausforderung! Gleichzeitig begann ich meine Promotion. Jetzt - acht Jahre später und fast pünktlich zu deinem achten Geburtstag - bin ich endlich am Ziel angekommen. Ich liebe Dich sehr. genauso wie meinen heißbütigen Geschichtenerzähler Paul und unseren Sonnenschein Emil. Ich bin stolz euer Vater zu sein! 


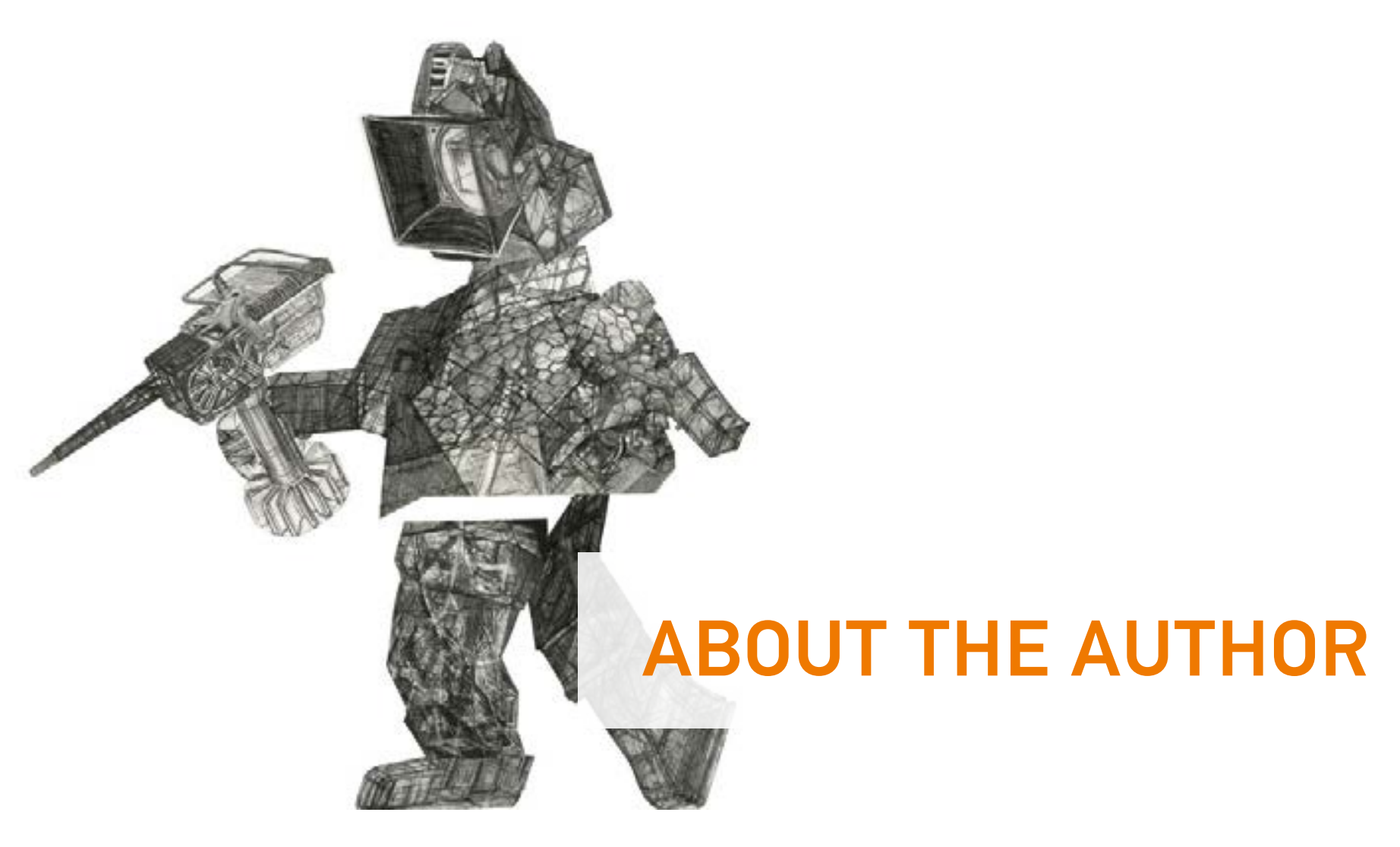




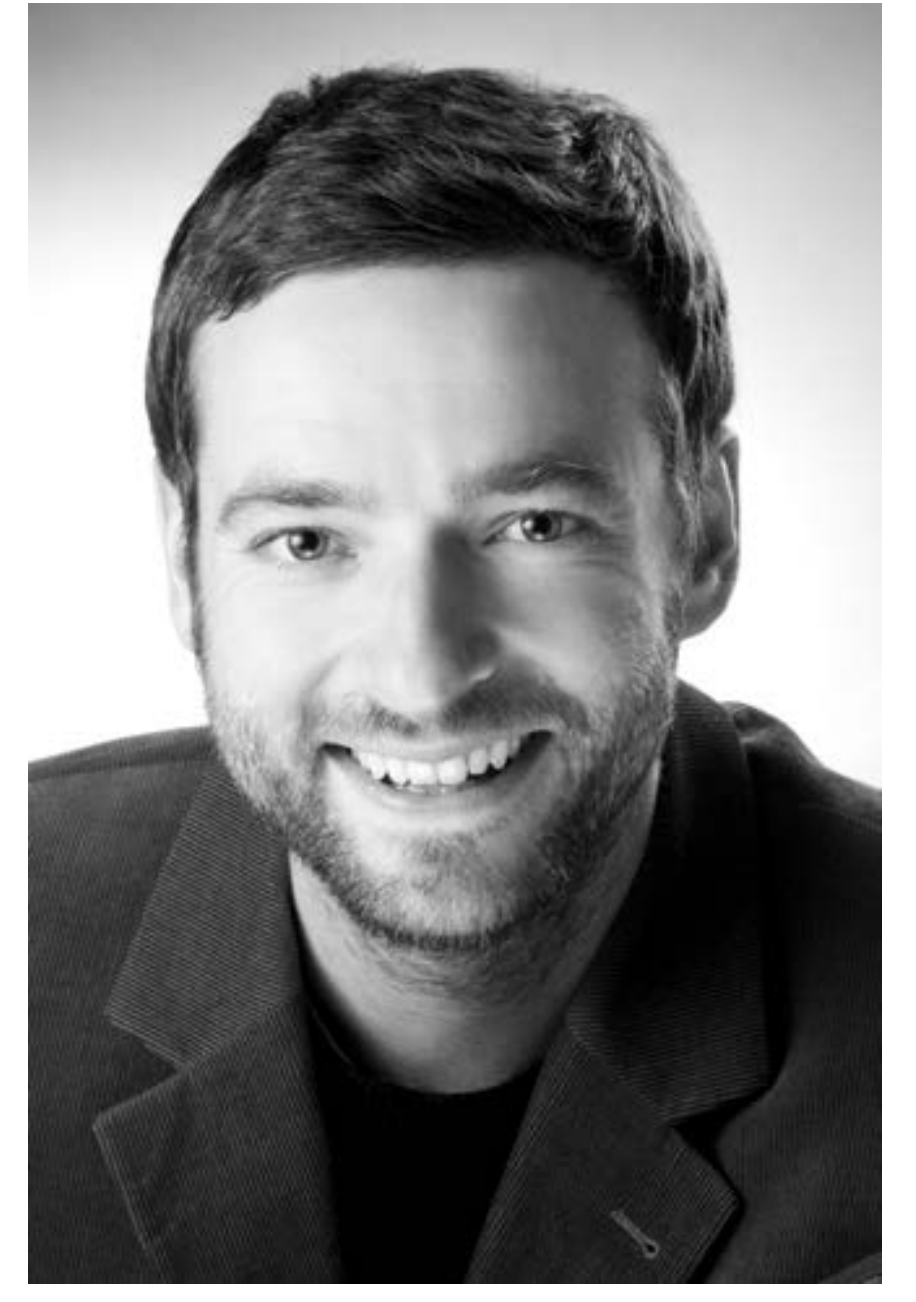

Andreas Rothgangel was born as the younger brother of two sons on May 2nd 1977 in Speyer, Germany. In 1996, after a happy childhood in Speyer, he graduated from secondary school ('Kalserdom Gymnasium,', Speyer). Afterwards, Andreas moved from the South of Germany to the Netherland to study physical therapy at Zuyd University of Applied Sciences Heerlen, where he graduated cum laude in 2002. In his bachelor thesis, he performed a randomized controlled trial about the effects of mirror therapy in stroke patients together with one of his best friends and paranymph Alexander Morton. Their thesis was awarded by the Royal Dutch Sociely for Physical Therapy (KNGF) in 2002 and published in the Dutch Journa After his study, Andreas started working as a physical therapist first in Aachen later on in Düsseldorf, where he also met his lovely wife Sarah at a dancing party. He also started to give seminars on the topic of miror therapy, which he still enjoys doing currently. In 2004 , he enrolled in the Master of Science program in Public Health (Epidemiology) at Maastricht University, the Netherlands and graduated in 2006 with his master thesis investigating the neurophysiological mechanisms of mirror therapy. After graduation, he started teaching at the School of Physical Therapy Düsseldorf and Fresenius University of Applied Sciences in Idstein. Finally, he returned to Zuyd University of Applied Sciences in 2009, where one of his main tasks since then is to supervise physical therapy students regarding their bachelor thesis.

In 2010, he started working part-time on his PhD-project on the use of mirror therapy in patients with phantom limb pain following amputation. NRW, German) and the Europen Union for his proposat on the PACT

project, which he wrote together with his PhD team Sandra Beurskens, Susy Braun, Rob Smeets and Luc de Witte. The PhD-project was embedded in the Research Centre for Autonomy and Participation of Persons with a Chronic lllness of Zuyd University of Applied Sciences and the Department of Rehabilitation Medicine, Maastricht University, The Netherlands which is part of the Research School CAPHRI. The PACT project was an industry-parthered PhD program, wherein Andreas worked part-lime as project leader for Kaasa health, a software company based in Dusseldorf, Germany. From 2015 on, he also stanted supervising anohth langer industry-partnered project on using virtual and augmented reality in patients with phantom limb pain. Currently, Andreas is working at the Research Center for Nutrition, Lifestyle and Exercise of Zuyd Uninersity of Appled in research and development of personalized digital health interventions.

Andreas lives with his lovely wife Sarah and their three beautiful boys in Düsseldorf, Germany. 


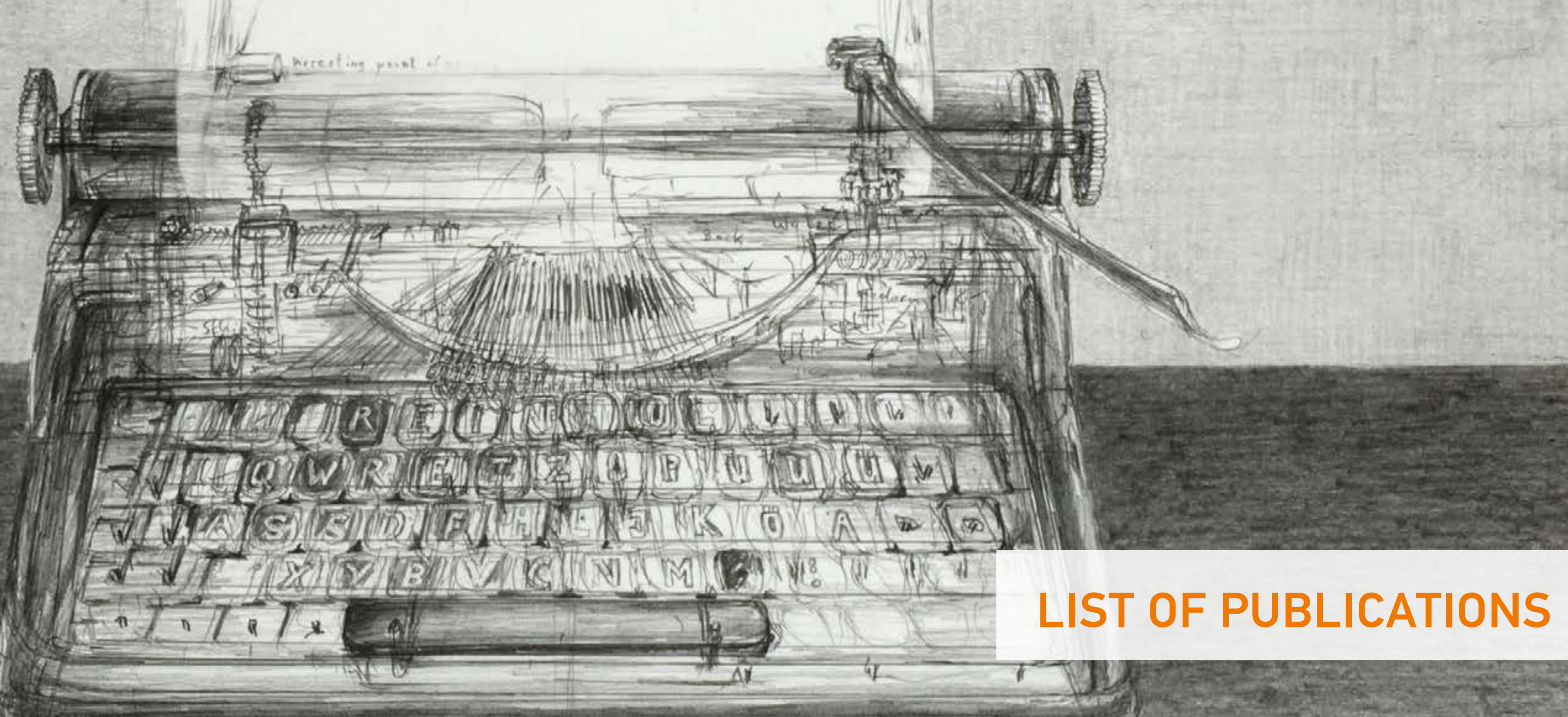




\section{Publications within this thesis}

Rothgangel AS, Braun SM, Beurskens AJ, Seitz RJ, Wade DT. The clinical aspects of mirror therapy in rehabilitation: a systematic review of the literature. Int J Rehabil Res 2011; 34(1):1-13.

Rothgangel AS, Braun S, Schulz RJ, Kraemer M, de Witte L, Beurskens A, Smeets RJ. The PACT trial: PAtient Centered Telerehabilitation effectiveness of software-supported and traditional mirror therapy in patients with phantom limb pain following lower limb amputation: protocol of a multicentre randomised controlled trial. J Physiother 2015; 61(1):42.

Rothgangel A, Braun S, de Witte L, Beurskens A, Smeets R. Development of a Clinical Framework for Mirror Therapy in Patients with Phantom Limb Pain: An Evidence-based Practice Approach. Pain Pract 2016; 16(4):422-34.

Rothgangel A, Braun S, Smeets R, Beurskens A. Design and Development of a Telerehabilitation Platform for Patients With Phantom Limb Pain: A User-Centered Approach. JMIR Rehabil Assist Technol 2017; 4(1):e2.

Rothgangel A, Braun S, Winkens B, Beurskens A, Smeets R. Traditional and augmented reality mirror therapy for patients with chronic phantom limb pain (PACT study): results of a three-group, multicentre single-blind randomized controlled trial. Clin Rehabil 2018; 32(12):1591-

Rothgangel A, Braun S, Smeets R, Beurskens A. Feasibility of a traditional and teletreatment approach to mirror therapy in patients with phantom limb pain: A process evaluation performed alongside a randomized controlled trial. Clin Rehabil 2019; May.

\section{Other publications of mirror therapy}

Rothgangel AS and Braun SM. Mirror Therapy: Practical Protocol for Stroke Rehabilitation. Munich: Pflaum Verlag, 2013.

Rothgangel A and Bekrater-Bodmann R. Mirror therapy versus augmented/virtual reality applications: towards a tailored mechanism-based treatment for phantom limb pain. Pain Manag 2019: 9(2):151-159.

\section{National publications}

作 study. Ned Tijdschr Fys 2004; 114(2):36-40.

Morton A, Rothgangel AS. Chronische Hemiparese: Spiegeltherapie wirkt. Physiopraxis 2004; 6:16.

Morton A, Rothgangel AS. Illusion mit Erfolg: Spiegettherapie. Physiopraxis 2004; 11/12:44-47.

Rothgangel AS. Spiegeltherapie: Training the brain. ProManu 2007;2.

Rothgangel AS. Mit Spiegeltherapie gegen Phantomschmerzen - theoretisch wissenschaftlicher Hintergrund und praktische Anwendung Neur Psych Nachr 2007; 3/4.

Rothgangel AS, Morton AR, van den Hout JWE, Beurskens AJHM. Spiegeltherapie in der Neurologischen Rehabilitation: Effektivität in Bezug auf die Arm- und Handfunktionen bei chronischen Schlaganfallpatienten. Neurol Rehabil 2007; 13(5):271-276.

Rothgangel AS. Geplagte Füße in neuer Form. Clinical Reasoning und evidenzbasierte Behandlung der M. tibialis posterior-Sehnendysfunktion. pt_Zeitschrift für Physiotherapeuten 2007; 59(12):1154-58.

Rothgangel AS. Dem Sporn auf den Fersen. Clinical Reasoning und evidenzbasierte Behandlung der Fasziitis plantaris. pt_Zeitschrift für Physiotherapeuten 2008; 60(1):68-74.

Rothgangel AS. Die Achillesferse. Clinical Reasoning und evidenzbasierte Behandlung der Achilles-Tendinopathie. pt_Zeitschrift für Physiotherapeuten 2008; 60(2):203-208

Rothgangel AS. Muskeln unter Druck - ist die operative Versorgung der einzige Ausweg? Clinical Reasoning und evidenzbasierte Behandlung des funktionellen Kompartmentsyndroms. pt_Zeitschrift für Physiotherapeuten 2008; 60(3):324-329. 
Rothgangel AS. Das Läuferknie. Clinical Reasoning und evidenzbasierte Behandlung des lliotibialband-Syndroms. pt_Zeitschrift für Physiotherapeuten 2008; 60 (5):540-44.

Rothgangel AS. Patello-femorales Schmerzsyndrom - Clinical Reasoning und evidenzbasierte Behandlung. pt_Zeitschrift fur Physiotherapeuten 2008; 60(9):998-1004.

Rothgangel AS. Spiegettherapie - mehr als nur eine visuelle Illusion? Aktuelle Übersicht über neurophysiologische Hintergründe und Anwendungsmöglichkeiten. pt_Zeitschrift für Physiotherapeuten 2008; 60(11):1243-1249.

Rothgangel AS. Spiegeltherapie - eine sinnvolle Ergänzung nicht nur für die neurologische Rehabilitation. Praxis Physiotherapie 2009;(3):32-

Mey M, Herzing K, Braun SM, Rothgangel AS. Virtuelle Rehabilitation bei Parkinson: Einsatz einer Videospiel-Konsole in de physiotherapeutischen Behandlung. pt_Zeitschrift für Physiotherapeuten 2010; 62(7):6-13.

Hofmann B, Rothgangel AS. Evaluation eines klinischen Protokolls zur Klassifzierung und Behandlung von Patienten mit patellofemoralem Schmerzsyndrom: 3 Einzelfallstudien. Manuelle Therapie 2010; 14: 110-117.

Drauz T, Rothgangel AS, Braun SM. Externales Feedback in der Physiotherapie. pt_Zeitschrift für Physiotherapeuten 2011; 63(6): 74-77. Genius J, Roß S, Uhr S, Braun SM, Rothgangel AS. Praxisleitfaden für die Anwendung der Spiegeltherapie bei neurologischen Patienten pt_Zeitschrift für Physiotherapeuten 2012; 64(5):42-45.

Genius J, Roß S, Uhr S, Braun SM, Rothgangel AS. Spiegeltherapie - Praxisleitfaden Neurologie. Richard Pflaum Verlag, München 2012. Rothgangel A, Braun S, Beurskens S, Greitemann B, Schröter J, de Witte L, et al. Mit dem Tablet-PC gegen Phantomschmerzen. Deutsches Artzeblatt 2015: (4):16-9.
Rothgangel $A$. Spielend gegen den Schmerz trainieren: Virtuelle und erweiterte Realität zur Behandlung von Patienten mit Phantomschmerzen pt_Zeitschriff für Physiotherapeuten 2017; 69(1):28-32.

Rothgangel A, Kanera I, Lenssen T, Braun S. Telemonitoring nach Ruptur des vorderen Kreuzbands. pt_Zeitschrift für Physiotherapeuten 2018; 70(2):20-25.

Kanera I, Rothgangel A, Schröen Y, Braun S. Zivilisationskrankheiten in den Griff bekommen? Ein personalisierter systembiologischer Ansatz pt_Zeitschriff für Physiotherapeuten 2019; $71(2)$

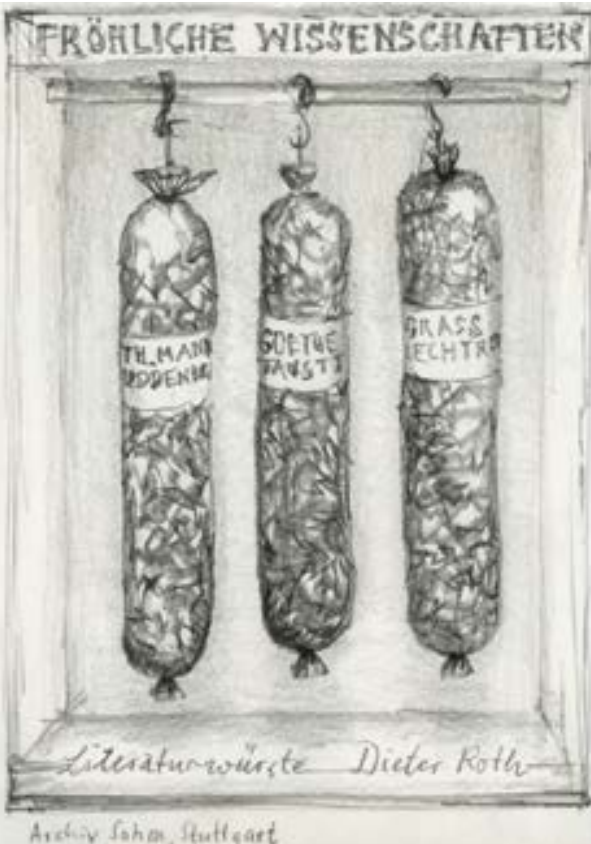




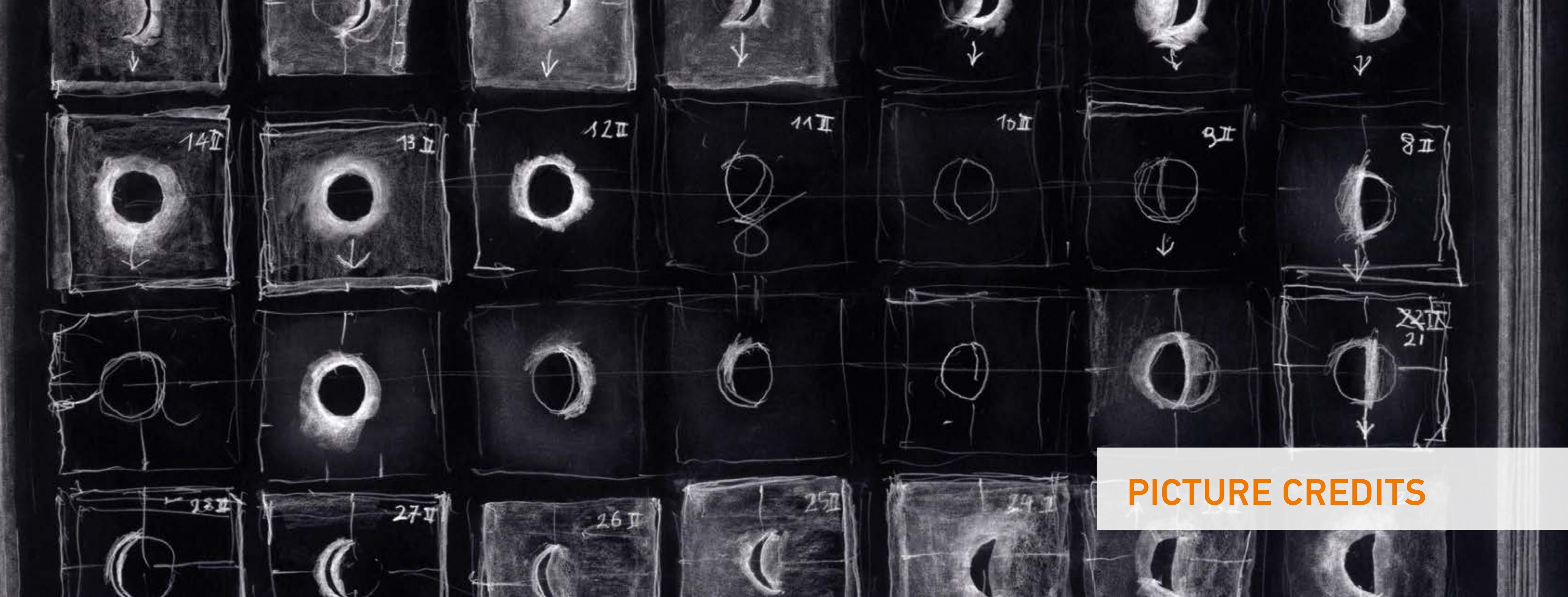




\section{MATTHIAS REINHOLD}

Mathias Reinhold wurde 1978 im Alb-Donau-Kreis geboren, studierte von 1998 bis 2004 an der Kunstakademie Stuttgart bei Wolfgang Gäfgen und Alexander Roob, wohnte dann in China, Warschau, Paris, Venedig, Ulm, Langenargen und Berlin, wo er heute mit Partnerin und zwei Söhnen lebt. 2015-18 brachte er angehenden Architekten an der ETH Zürich das Zeichnen näher. Er arbeitet je nach Witterung und Laune draußen auf dem Feld, auf der Straße und in Museen und zu Hause in der Küche. Sein Bildernetzwerk ikonolog entspinnt sich in Ausstellungen, Heften und im Internet.

\section{www.ikonolog.de}

\section{BILDER}

Brennendes Streichholz, $2013(21 \times 29,7 \mathrm{~cm}$, Bleistift auf Papier)

Streichholzschachtel, $2008(21 \times 29,7 \mathrm{~cm}$, Bleistift auf Papier)

Taucher, 2008 (21 × $29,7 \mathrm{~cm}$, Bleistift auf Papier)

Charles Darwin, $2011(21 \times 29,7 \mathrm{~cm}$, Bleistift auf Papier)

Dieter Roth Literaturwürste, $2015(21 \times 14,8 \mathrm{~cm})$

Bodenschatze, $2008(24 \times 33 \mathrm{~cm})$

Zivilisation, $2008(243 \times 148 \mathrm{~cm}$, Kohle auf Papier)

Laptop i, $2006(150 \times 200 \mathrm{~cm}$, Kohle und Tusche auf Papier $)$

Cannochiale Aristotelico, 2013, $(29,7 \times 42 \mathrm{~cm})$
Zelt, $2008(21 \times 29,7 \mathrm{~cm}$, Bleistift auf Papier)

Zielscheibe, $2011(48 \times 33 \mathrm{~cm})$

Muntadas Kerze Glühbirne, $2013(15 \times 10 \mathrm{~cm}$, digital invertiert)

Fenster Berglandschaft, 2009 (Digitalcollage)

Fenster, $2008(24 \times 33 \mathrm{~cm})$

Landschaft (Rahmen), $2008(24 \times 33 \mathrm{~cm})$

Wasserkreislauf Baum, $2016(21 \times 29,7 \mathrm{~cm}$, Bleistift auf Papier)

Havarie, (148 $200 \mathrm{~cm}$, Kohle auf Papier)

Streichhölzer (Gesellschaft), $2012(15 \times 21 \mathrm{~cm})$

Bohrmaschinerie, 2006 (Kohle auf Papier, $150 \times 320 \mathrm{~cm}$ )

Neumond (Micha Ullman), 2016 (digital invertiert) 
